\title{
Alzheimer's disease through the MR-eye; novel diagnostic markers and the road to clinical implementation
}

Citation for published version (APA):

Clerx, L. (2014). Alzheimer's disease through the MR-eye; novel diagnostic markers and the road to clinical implementation. [Doctoral Thesis, Maastricht University]. NeuroPsych Publishers. https://doi.org/10.26481/dis.20140516lc

Document status and date:

Published: 01/01/2014

DOI:

10.26481/dis.20140516lc

Document Version:

Publisher's PDF, also known as Version of record

\section{Please check the document version of this publication:}

- A submitted manuscript is the version of the article upon submission and before peer-review. There can be important differences between the submitted version and the official published version of record.

People interested in the research are advised to contact the author for the final version of the publication, or visit the DOI to the publisher's website.

- The final author version and the galley proof are versions of the publication after peer review.

- The final published version features the final layout of the paper including the volume, issue and page numbers.

Link to publication

\footnotetext{
General rights rights.

- You may freely distribute the URL identifying the publication in the public portal. please follow below link for the End User Agreement:

www.umlib.nl/taverne-license

Take down policy

If you believe that this document breaches copyright please contact us at:

repository@maastrichtuniversity.nl

providing details and we will investigate your claim.
}

Copyright and moral rights for the publications made accessible in the public portal are retained by the authors and/or other copyright owners and it is a condition of accessing publications that users recognise and abide by the legal requirements associated with these

- Users may download and print one copy of any publication from the public portal for the purpose of private study or research.

- You may not further distribute the material or use it for any profit-making activity or commercial gain

If the publication is distributed under the terms of Article $25 \mathrm{fa}$ of the Dutch Copyright Act, indicated by the "Taverne" license above, 
Alzheimer's disease through the MR-eye Novel diagnostic markers and the road to clinical implementation 
Copyright (C) by Lies Clerx, Maastricht 2014

Cover design: Steven Schenk

Layout: Lies Clerx

Printing: Boxpress NV

Publisher: NeuroPsych Publishers

ISBN: 978-90-75579-68-0

All rights reserved. No part of this thesis may be reproduced, stored or transmitted in any way or by any means without the prior permission of the author or, when appropriate, from the publishers of the publications. 


\section{Alzheimer's disease through the MR-eye}

Novel diagnostic markers and the road to clinical implementation

\section{PROEFSCHRIFT}

Ter verkrijging van de graad van doctor aan de Universiteit Maastricht, op gezag van de Rector Magnificus, Prof. dr. L.L.G. Soete, volgens het besluit van het college van Decanen, in het openbaar te verdedigen op

16 mei 2014 om $12 u$

door

Lies Clerx 


\section{Promotor:}

Prof. dr. F.R.J. Verhey

\section{Co-promotores:}

Dr. P. Aalten

Dr. P.J. Visser

\section{Beoordelingscommissie:}

Prof. dr. Y. Temel (voorzitter)

Prof. dr. M. Vandenbulcke (Katholieke Universiteit Leuven, België)

Prof. dr. S. Teipel (University of Rostock, Germany)

Dr. P. Hofman

Dr. I. Ramakers

The research described in this thesis was performed at the Department of Psychiatry \& Neuropsychology, School for Mental Health and Neuroscience, Maastricht University, The Netherlands.

Financial support for the publication of this thesis was kindly provide by Alzheimer Nederland, Lundbeck, CTMM, Internationale Stichting Alzheimer Onderzoek (ISAO), Nutricia Advanced Medical Nutrition and Science Plus Group.

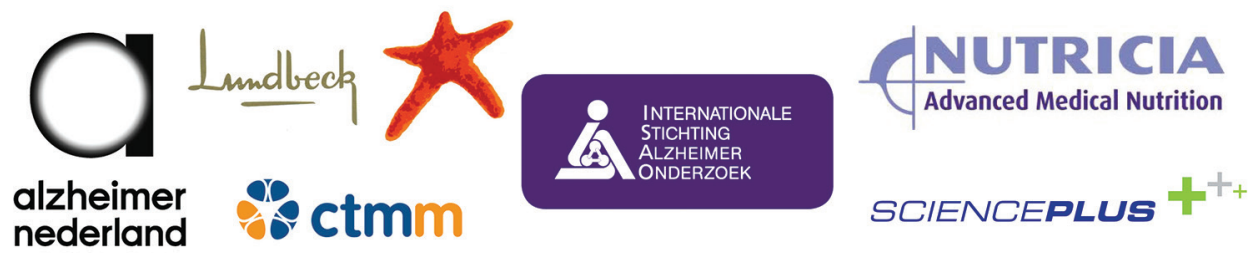


voor Marie-Lou en Maurice, From here to the moon and back Who else in this world will love you like that? Forever and always, I'll be where you're at From here to the moon and back. From here to the moon and back. 



\section{CONTENTS}

Chapter 1 General introduction

Part I: Grey matter atrophy in (early) AD

Chapter 2 The added value of MRI-biomarkers to

neuropsychological test performance for the prediction

of $A D$ in subjects with $\mathrm{MCl}$

Submitted

Chapter 3 Measurements of medial temporal lobe atrophy for

prediction of $A D$ in subjects with $\mathrm{MCl}$

Neurobiol Aging. 2013 Aug;34(8):2003-13

Chapter 4 Sensitivity of different MRI-techniques to assess

89

grey matter atrophy patterns in Alzheimer's disease is region-specific

Curr Alzheimer Res. 2013 Nov;10(9):940-51

\section{Part II: Structural connectivity in (prodromal) AD}

Chapter 5 New MRI markers for Alzheimer's disease:

a meta-analysis of diffusion tensor imaging and a comparison with medial temporal lobe measurements J Alzheimers Dis. 2012;29(2):405-29

Chapter 6 Reduced callosal white matter integrity surpasses cerebrospinal fluid and atrophy markers as predictor of cognitive decline in subjects with $\mathrm{MCl}$ : a combined volumetry and DTI study

Submitted 

Part III: Robustness of structural and functional abnormalities in AD

Chapter 7 Consistency of FreeSurfer regional atrophy

measurements compared to manual measurements

in Alzheimer's disease

Submitted

Chapter 8 The stability of resting state networks over time in

healthy old and young subjects and its relevance

for early memory dysfunction

Submitted

Chapter 9 General discussion

275

Future directions

Appendix

Summary

295

Nederlandse samenvatting

301

Curriculum vitae

307

List of publications

309

Dankwoord 


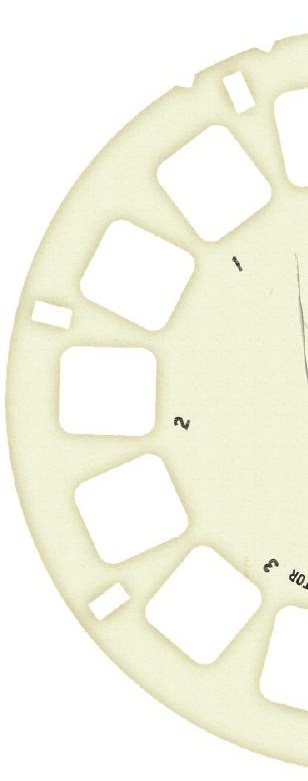




\section{Chapter 1 \\ General Introduction}

$\uparrow$

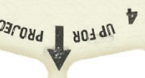

c)

v 


\section{Purpose and motivation}

Due to the aging of the population, Alzheimer's disease (AD) is a growing socioeconomic problem. The underlying changes associated with this neurodegenerative disease start years before clinical manifestation [1]. On a pathological level, ADpatients show an accumulation of amyloid plaques and neurofibrillary tangles which are associated with loss of neurons [2]. Potential disease modifying therapies should be given in the earliest stage of the disease, to prevent further neuronal loss. To date, no single diagnostic marker is available with sufficiently high predictive accuracy rates to provide an accurate diagnosis in a single patient. Consequently, there is a need for novel markers to detect the disease at a preclinical stage. Magnetic Resonance Imaging (MRI) provides a framework to visualize structural and functional changes associated with neurodegenerative diseases in vivo. The use of neuroimaging in clinical practice has shifted from exclusion of other diseases that may cause the cognitive impairment (e.g. tumor, hemorrhage) to early detection (e.g. AD versus healthy aging) and differential diagnosis (e.g. AD versus frontotemporal dementia).

The current thesis aims to expand insight into the use of both conventional (e.g. hippocampal volumetry) and novel (e.g. diffusion tensor imaging) MR imaging markers for early detection, diagnosis and prognosis of AD. Associations with clinical and cerebrospinal fluid (CSF) biomarkers are explored to further disentangle the underlying nature and course of the disease.

\section{GENERAL INTRODUCTION}

Alzheimer's disease and $\mathrm{MCl}$

Alzheimer's disease (AD) is a progressive neurodegenerative disorder affecting approximately 250.000 peopleintheNetherlands, and 35 million individualsworldwide [3]. Despite numerous research, $A D$ is currently incurable and the number of patients is expected to exceed 115 million by 2050 . The term mild cognitive impairment $(\mathrm{MCl})$ has been introduced to describe patients who do not fulfill clinical criteria for dementia, but who do have objective evidence of memory deficits without notable interference with daily life activities [4]. $\mathrm{MCl}$ patients carry an increased risk of developing $A D$, with about $10-15 \%$ progressing to $A D$ per year in academic 
clinical settings. In these subjects, $\mathrm{MCl}$ is considered to be a transitional phase. However, not all $\mathrm{MCl}$-patients progress to $\mathrm{AD}$ : some develop another type of dementia, while others improve or remain stable [5]. The course of $A D$ is also variable: not all patients progress at the same rate and the factors influencing or predicting progression are not well known [6]. Better modeling of disease progression in individual subjects will allow us to predict when cognitive abilities may break down and when social and behavioral disturbances may arise. Staging models based on dynamic biomarkers (Figure 1)[7] will furthermore allow us to evaluate treatment effects of novel drugs in clinical trials based on the effects of a specific drug in slowing down or stopping transition from one stage to the next.

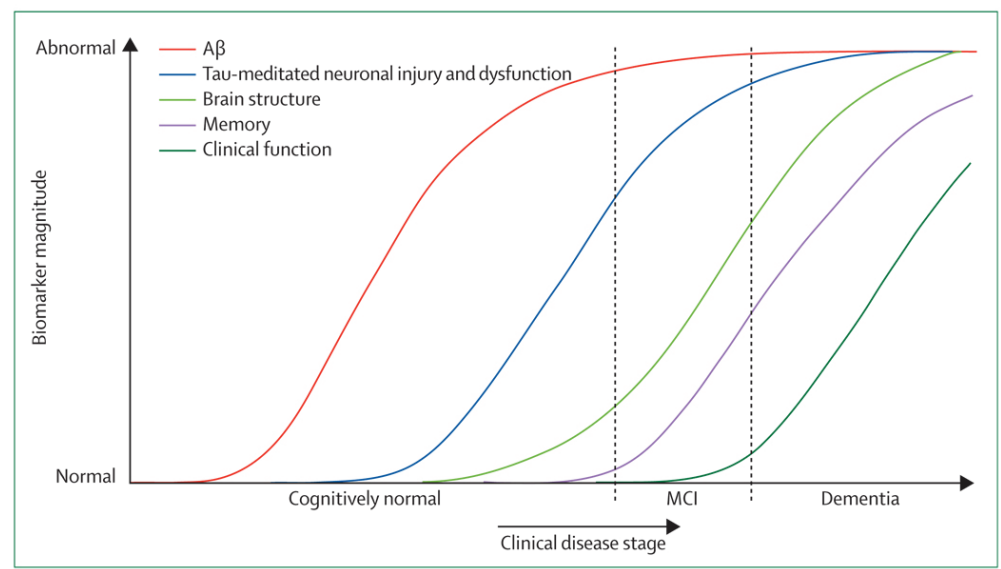

Figure 1. Hypothetical model of imaging biomarkers in Alzheimer's disease [7].

\section{Diagnosing AD}

\section{Pathophysiology}

The neuropathological hallmarks of $A D$ are extracellular senile plaques consisting of amyloid-beta $(A \beta)$ and intracellular neurofibrillary tangles consisting of hyperphosporylated tau, both associated with neuronal loss. $A \beta$ is a peptide produced by cleavage of the $A \beta$ precursor protein (APP) [8] and deposition of amyloid plaques is widely distributed throughout the cortex, starting in the posterior association cortices, and showing a relative sparing of the medial temporal lobe (MTL) [2]. The 'amyloid cascade hypothesis' 
was recently postulated in which $A \beta$ starts to accumulate approximately 15 years before the onset of clinical symptoms [7, 9]. Formation of neurofibrillary tangles on the other hand, is postulated to start in the MTL and spreads into adjacent lateral temporal and association parietal cortices, followed by prefrontal cortices and other neocortical areas (e.g. motor and sensory areas) in later stages of the disease [10]. Plaques and tangles are associated with regional neuronal loss, cortical atrophy and cognitive decline $[2,10]$. Both hallmarks of AD can most accurately be quantified post-mortem, or in vivo by means of CSF analysis or beta-amyloid Positron Emission Tomography (PET) imaging [11].

\section{Clinical assessment}

A clinical diagnosis of $\mathrm{MCl}$ or dementia involves assessing cognitive functions as well as the degree of interference in daily life activities, psychological well-being and burden of care. These elements can be evaluated by means of an interview with both patient and caregiver(s), and neuropsychological assessment. The latter one plays a major role in determining the nature and extent of the cognitive dysfunction. Additional investigations are needed to clarify the underlying cause of the cognitive impairment. Some causes can be identified by means of a blood test or neurological examination, but for most neurodegenerative disorders imaging or CSF assessment are needed.

\section{Macrostructural neuroimaging}

MRI or computed tomography (CT) are routinely used to exclude other diseases that can cause the cognitive impairment. They can also provide positive evidence for AD. Such studies mainly focussed on the MTL, which is affected early in the disease process. However, atrophy of the MTL is non-specific for AD [12] and tissue loss is not limited to this region [13]. To evaluate the degree of atrophy in the MTL, clinicians prefer the use of visual rating scales because these instruments are readily available, quick and easy to perform [14]. However, visual rating scales show lower predictive accuracy rates and higher inter and intraindividual variability effects [15] compared to volumetric (hippocampal) measurements. Manual volumetry is still considered gold standard in research practice [16-18], but is time consuming which thereby limits routine clinical or large-scale research use. Automated hippocampal measurement needs much less rater time and shows no interrater variability effects, but might be susceptible to scanner and scan protocol variability. 


\section{Novel imaging markers}

Novel imaging markers have now been developed to evaluate changes in gray (GM) and white matter (WM), both on a structural (e.g. diffusion tensor imaging (DTI)) and functional level (e.g. resting state functional MRI). These markers can be used to test structural and functional connectivity between brain regions. Structural markers are able to visualize physical connectivity between brain regions, while functional connectivity markers display functional connections between brain regions that are not necessary directly physically connected. In early AD, both structural and functional connectivity are altered [19-24] and there is some evidence that these measures become abnormal before macroscopic atrophy is evident. Thus, by means of these techniques, structural disconnection may be detected at a stage where macrostructural changes such as hippocampal atrophy may not have become visible yet, and are linked with neuropsychological performance $[25,26]$.

\section{Structural connectivity markers}

DTI measures the random motion of water molecules within tissue, reflecting structural organization. Diffusion anisotropy (FA) is often regarded as an index reflecting microscopic tissue integrity and correlates with the integrity of the axonal membrane [27]. Reduction of FA values suggests a reduction in axon number, an impairment of axonal flow, or both. An index of the magnitude of diffusion independent of the direction is mean diffusivity (MD). An increase in MD is thought to reflect enlargement in the extracellular space due to altered cytoarchitecture (i.e. loss of neurons, axons, and dendrites), suggesting immaturity or degeneration of the tissue leading to elevated water diffusivity within these regions $[28,29]$. Alterations in FA or MD could also be due to diffusion changes either parallel or perpendicular to the principal direction of the tensor. These measurements are referred to as axial (AXD), and radial diffusivity (RAD), respectively. An increase in RAD is thought to signify increased space between fibers (suggesting demyelination or dysmyelination) [30] whereas increases in AXD suggest axonal damage [31].

\section{Functional connectivity markers}

Functional connectivity can be tested using the BOLD signal in task-related functional or resting-state MRI paradigms [32-34]. Functional connections can be derived from their temporal pattern in neurophysiologic change or activation during task-related 
or task-free research environments [35]. Task-related decreases are found in certain regions which remain active in an organized fashion during the resting state of the brain $[36,37]$. One of these 'resting state networks' is the default mode network which is particularly relevant for aging and dementia since its vulnerability to atrophy, deposition of the amyloid protein, and reduced glucose metabolism [38].

\section{Biomarkers in cerebrospinal fluid (CSF)}

CSF biomarkers are increasingly used to detect brain changes associated with $A D$ in vivo. In CSF, decreases in levels of beta-amyloid 1-42 (A $\beta$ 1-42) are found, together with increases in total ( $\mathrm{t}$-tau) and phosphorylated tau ( $\mathrm{p}$-tau). As the presence of these protein levels reflects $A D$ pathology, these CSF biomarkers have been shown to differentiate patients with AD from control subjects [39] and to predict conversion rate in $\mathrm{MCl}$-patients [40]. Both MRI and CSF are valuable markers of disease [41], however, the relation between these markers has been less extensively studied.

\section{To cure or not to cure?}

Despite tremendous research, $A D$ is currently incurable. Cholinesterase inhibitors are most widely used to reduce the behavioural disorders associated with the disease [42]. Non-pharmalogical interventions are often additionally implemented to further reduce behavioural disturbances, and to reduce caregiver burden [43, 44].

\section{Aims and outline}

MR imaging provides more information than macrostructural atrophy and vascular abnormalities. However, the added value and exact position of novel imaging techniques in the diagnostic field of $A D$ remains unclear. We will investigate whether MRI investigation is necessary in the diagnostic process of $A D$ and which MRI measurements can incrementally contribute to the early and specific diagnosis of Alzheimer disease. The overall aim of this thesis is to evaluate the clinical value of novel imaging markers for $A D$ in relation to current (gold standard) methods (i.e. hippocampal volumetry, visual rating scale). The first part of the thesis evaluates well-known imaging techniques which assess gray matter atrophy in the brain. In the second part we will examine the discriminative and predictive value of a novel imaging marker for AD: Diffusion Tensor Imaging (DTI). In the third part, we will investigate the robustness of structural and functional abnormalities in AD. 


\section{Macrostructural imaging}

The current approach in most of Dutch memory clinics consists of cognitive examination of patients by means of a neuropsychological test battery, together with a neurological investigation, (hetero)anamnesis, a blood test and an MRI scan. This clinical protocol forms the starting point of the thesis:

1. 'What is the added value of volumetric MRI to neuropsychological test performance?'

In Chapter 2, we investigate if MR imaging markers increase predictive accuracy for $A D$ relative to a model that only includes demographical and neuropsychological information. Furthermore, the importance of MTL atrophy in $A D$ allows us to formulate a second question:

2. 'Which measurement of MTL atrophy is the most sensitive to predict $A D$-conversion in subjects with $M C l$ ?'.

Chapter 3 investigates the predictive value of four different MTL atrophy measurements for conversion to $A D$ in subjects with $\mathrm{MCl}$. Two questions result from recent evidence stating that $A D$ pathology is not restricted to the MTL:

3. 'Which regions in the brain can discriminate between controls, $\mathrm{MCl}$ and $A D$ ?'

$\&$

4. 'Are different techniques assessing gray matter atrophy in the brain leading to the same pattern of abnormalities throughout the same dataset?'

Chapter 4 describes the discriminative value of several techniques measuring GM atrophy in the brain, both in and outside medial temporal lobe regions. 


\section{Connectivity markers}

The second part of the thesis examines the discriminative and predictive value of a novel imaging marker for AD: DTI. The main questions are:

\section{5. 'What is the added value of DTI in discriminating healthy controls, $\mathrm{MCl}$ and $A D$ ?'}

\&

\section{6. 'Is DTI superior to MTL measurements?'.}

Chapter 5 will assess the value of DTI as imaging marker for AD in relation to MTL measurements by means of a meta-analysis. The same question will be addressed in chapter 6 by means of a case-control study. Differences in both gray matter atrophy and WM integrity are evaluated in a multi-center cohort of healthy old subjects, $\mathrm{MCl}$ and AD-patients. The predictive value of both volumetry and DTI for cognitive decline at 2 years follow-up is furthermore investigated.

\section{Methodological issues}

The third and last part of the thesis investigates the robustness of structural and functional abnormalities in AD. The main question is:

7. 'Are structural and functional imaging techniques influenced by internal (e.g. patients age) and external (e.g. software, hardware) factors?'

Chapter 7 examines the consistency of FreeSurfer, an automated segmentation tool, to detect structural abnormalities under varying processing conditions, and in relation to manual volumetry.

Chapter 8 investigates the validity of resting state $\mathrm{fMRI}$ as a marker for disease, by evaluating the stability or test-retest reproducibility of resting state networks over time in a healthy population.

Chapter 9 presents our overall conclusions and puts the main findings presented in this thesis in the context of our current knowledge about AD. 


\section{REFERENCES}

1. Petersen RC, Doody $R$, Kurz A, et al. Current concepts in mild cognitive impairment. Arch Neurol 2001;58:1985-1992.

2. Braak H, Braak E. Neuropathological stageing of Alzheimer-related changes. Acta Neuropathologica 1991;82:239-259.

3. 2013 Alzheimer's disease facts and figures. Alzheimer's \& Dementia 2013;9:208-245.

4. Petersen RC, Smith GE, Waring SC, Ivnik RJ, Tangalos EG, Kokmen E. Mild Cognitive Impairment: Clinical Characterization and Outcome. Archives of Neurology 1999;56:303-308.

5. Visser PJ, Kester A, Jolles J, Verhey F. Ten-year risk of dementia in subjects with mild cognitive impairment. Neurology 2006;67:1201-1207.

6. Fox NC, Scahill RI, Crum WR, Rossor MN. Correlation between rates of brain atrophy and cognitive decline in AD. Neurology 1999;52:1687-1689.

7. Jack CR, Knopman DS, Jagust WJ, et al. Hypothetical model of dynamic biomarkers of the Alzheimer's pathological cascade. The Lancet Neurology 2010;9:119-128.

8. Haass C, Selkoe DJ. Cellular processing of beta-amyloid precursor protein and the genesis of amyloid beta-peptide. Cell 1993;75:1039-1042.

9. Bateman RJ, Xiong C, Benzinger TLS, et al. Clinical and Biomarker Changes in Dominantly Inherited Alzheimer's Disease. New England Journal of Medicine 2012;367:795-804.

10. Braak H, Braak E. Development of Alzheimer-related neurofibrillary changes in the neocortex inversely recapitulates cortical myelogenesis. Acta Neuropathologica 1996;92:197-201.

11. Phelps ME. Positron emission tomography provides molecular imaging of biological processes. Proceedings of the National Academy of Sciences 2000;97:9226-9233.

12. Barber RM, Gholkar AF, Scheltens PM, Ballard CM, McKeith IGM, O'Brien JTD. Medial temporal lobe atrophy on MRI in dementia with Lewy bodies. Neurology 1999;52:1153-1158.

13. Jacobs HI, Van Boxtel MP, Uylings HB, Gronenschild EH, Verhey FR, Jolles J. Atrophy of the parietal lobe in preclinical dementia. Brain Cogn 2011;75:154163. 
14. Scheltens P, Leys D, Barkhof F, et al. Atrophy of medial temporal lobes on MRI in "probable" Alzheimer's disease and normal ageing: diagnostic value and neuropsychological correlates. J Neurol Neurosurg Psychiatry 1992;55:967972.

15. DeCarli CM, Kaye JAM, Horwitz BP, Rapoport SIM. Critical analysis of the use of computer-assisted transverse axial tomography to study human brain in aging and dementia of the Alzheimer type. Neurology 1990;40:872-883.

16. Barnes J, Ourselin S, Fox NC. Clinical application of measurement of hippocampal atrophy in degenerative dementias. Hippocampus 2009;19:510516.

17. Boccardi M, Ganzola R, Bocchetta M, et al. Survey of Protocols for the Manual Segmentation of the Hippocampus: Preparatory Steps Towards a Joint EADCADNI Harmonized Protocol. Journal of Alzheimer's Disease 2011;26:61-75.

18. van de Pol LA, van der Flier WM, Korf ESC, Fox NC, Barkhof F, Scheltens $P$. Baseline predictors of rates of hippocampal atrophy in mild cognitive impairment. Neurology 2007;69:1491-1497.

19. Haris M, McArdle E, Fenty M, et al. Early Marker for Alzheimer's Disease: Hippocampus T1rho Estimation. Journal of Magnetic Resonance Imaging 2009;29:1008-1012.

20. Jacobs HI, Van Boxtel MP, Heinecke A, et al. Functional integration of parietal lobe activity in early Alzheimer disease. Neurology 2012;78:352-360.

21. Risacher SL, Shen L, West JD, et al. Longitudinal MRI atrophy biomarkers: Relationship to conversion in the ADNI cohort. Neurobiology of Aging 2010;31:1401-1418.

22. Schoonenboom NSM, van der Flier WM, Blankenstein MA, et al. CSF and MRI markers independently contribute to the diagnosis of Alzheimer's disease. Neurobiology of Aging 2008;29:669-675.

23. Tondelli M, Wilcock GK, Nichelli P, De Jager CA, Jenkinson M, Zamboni G. Structural MRI changes detectable up to ten years before clinical Alzheimer's disease. Neurobiology of Aging 2012;33:825-836.

24. Westman E, Cavallin L, Muehlboeck JS, et al. Sensitivity and Specificity of Medial Temporal Lobe Visual Ratings and Multivariate Regional MRI Classification in Alzheimer's Disease. PLoS ONE 2011;6.

25. Binnewijzend MAA, Schoonheim MM, Sanz-Arigita E, et al. Resting-state fMRI changes in Alzheimer's disease and mild cognitive impairment. Neurobiology 
of Aging 2012;33:2018-2028.

26. Jacobs HIL, Gronenschild EHBM, Evers EAT, et al. Visuospatial processing in early Alzheimer's disease: A multimodal neuroimaging study. Cortex; a journal devoted to the study of the nervous system and behavior 2012.

27. Beaulieu C. The basis of anisotropic water diffusion in the nervous system -a technical review. NMR in Biomedicine 2002;15:435-455.

28. Sykova E. Extrasynaptic volume transmission and diffusion parameters of the extracellular space. Neuroscience 2004;129:861-876.

29. Kantarci KM, Petersen RCMDP, Boeve BFM, et al. DWI predicts future progression to Alzheimer disease in amnestic mild cognitive impairment. Neurology 2005;64:902-904.

30. Song S-K, Sun S-W, Ju W-K, Lin S-J, Cross AH, Neufeld AH. Diffusion tensor imaging detects and differentiates axon and myelin degeneration in mouse optic nerve after retinal ischemia. Neurolmage 2003;20:1714-1722.

31. Thomas B, Eyssen M, Peeters R, et al. Quantitative diffusion tensor imaging in cerebral palsy due to periventricular white matter injury. Brain 2005;128:25622577.

32. Fink GR, Markowitsch HJ, Reinkemeier M, Bruckbauer T, Kessler J, Heiss W-D. Cerebral Representation of One's Own Past: Neural Networks Involved in Autobiographical Memory. The Journal of Neuroscience 1996;16:4275-4282.

33. Kukolja J, Thiel CM, Eggermann T, Zerres K, Fink GR. Medial temporal lobe dysfunction during encoding and retrieval of episodic memory in nondemented APOE-e4 carriers. Neuroscience 2010;168:487-497.

34. Kukolja J, Thiel CM, Wilms M, Mirzazade S, Fink GR. Ageing-related changes of neural activity associated with spatial contextual memory. Neurobiology of Aging 2009;30:630-645.

35. Huettel SA, Song AW, McCarthy G. Functional Magnetic Resonance Imaging ( 2 ed.): Massachusetts: Sinauer, 2009.

36. Fox MD, Snyder AZ, Vincent JL, Corbetta M, Van Essen DC, Raichle ME. The human brain is intrinsically organized into dynamic, anticorrelated functional networks. Proceedings of the National Academy of Sciences of the United States of America 2005;102:9673-9678.

37. Greicius MD, Krasnow B, Reiss AL, Menon V. Functional connectivity in the resting brain: A network analysis of the default mode hypothesis. Proceedings of the National Academy of Sciences 2003;100:253-258. 
38. Buckner R, Snyder A, Shannon B, et al. Molecular, structural, and functional characterization of Alzheimer's disease: evidence for a relationship between default activity, amyloid, and memory. The Journal of neuroscience : the official journal of the Society for Neuroscience 2005;25:7709-7717.

39. Bouwman FH, Schoonenboom NSM, Verwey NA, et al. CSF biomarker levels in early and late onset Alzheimer's disease. Neurobiology of Aging 2009;30:1895-1901.

40. Mattsson N, Zetterberg $\mathrm{H}$, Hansson $\mathrm{O}$, et al. CSf biomarkers and incipient alzheimer disease in patients with mild cognitive impairment. JAMA 2009;302:385-393.

41. Vos S, van Rossum I, Burns L, et al. Test sequence of CSF and MRI biomarkers for prediction of AD in subjects with $\mathrm{MCl}$. Neurobiology of Aging 2012;33:22722281.

42. McGleenon, Dynan, Passmore. Acetylcholinesterase inhibitors in Alzheimer's disease. British Journal of Clinical Pharmacology 1999;48:471-480.

43. Cotelli M, Manenti R, Zanetti o, Miniussi C. Non-pharmacological intervention for memory decline. Frontiers in Human Neuroscience 2012;6.

44. Hogan DB, Bailey P, Black S, et al. Diagnosis and treatment of dementia: 5. Nonpharmacologic and pharmacologic therapy for mild to moderate dementia. Canadian Medical Association Journal 2008;179:1019-1026. 



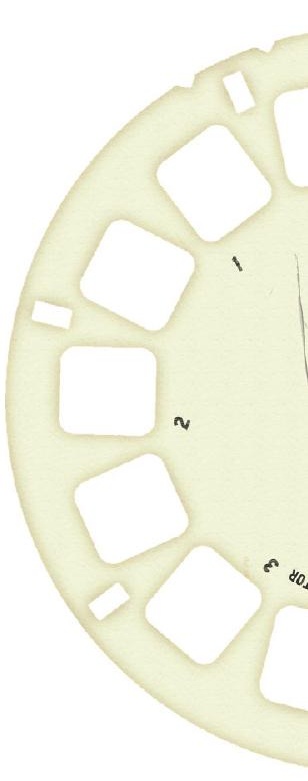




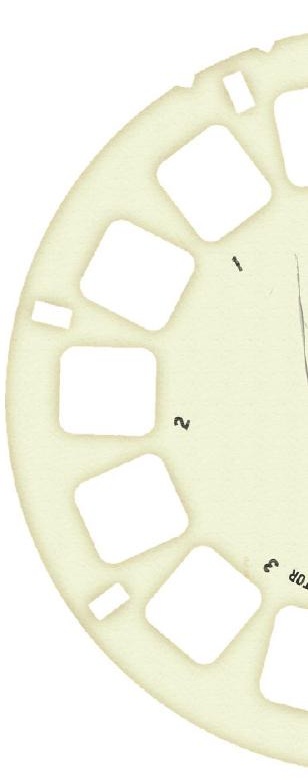




\section{Chapter 2}

The added value of MRI-biomarkers to neuropsychological test performance for the prediction of $A D$ in subjects

\section{with $\mathrm{MCl}$}

Lies Clerx, Eva Dierckx, Laura van de Pol, Ineke vain Rossum, Frans Verhey, Pauline Aalten, Frederik Barkhof, Robin Wolz, Daniel Rueckert, Giovanni B. Frisoni, Magdalini Tsolaki, Flavio Nobili, Yvonne Freund-Levi, Asa Wallin, Lutz Frölich, Harald Hampel, Hilkka Soininen, Philip Scheltens and Pieter Jelle Visser 


\section{ABSTRACT}

Background. Neuropsychological tests and magnetic resonance imaging (MRI) measures can identify Alzheimers's disease (AD) in the stage of mild cognitive impairment $(\mathrm{MCl})$. We aimed to investigate whether measurements of medial temporal lobe (MTL) atrophy could increase the predictive accuracy of neuropsychological tests for AD-type-dementia.

Methods. We selected $184 \mathrm{MCl}$-subjects from DESCRIPA and from the Alzheimer Dementia cohort of the VU University Medical center in Amsterdam. Neuropsychological tests for attention, memory, fluency, executive functioning and visuoconstruction were included. MTL atrophy measurements consisted of a visual rating scale and an automated hippocampal measurement. Outcome measure was the odds ratio for progression to AD-type dementia in 2 years.

Results. Among neuropsychological tests the odds ratio was highest for the wordlist delayed recall (3.59). The odds ratio was 4.14 for the MTA-score and 7.78 for hippocampal volume. Hippocampal volume but not MTA-score increased the predictive accuracy of delayed recall score for AD-type dementia after 2 years (AUC $=0.81 ; p<.001)$. Predictive accuracy was increased both in subjects with normal and abnormal delayed recall scores at baseline. In the subgroup of subjects with an MMSE score $>27$, both delayed recall and MRI at baseline were predictors of conversion. In the group of subjects with a normal MTA-score $(<3)$, only volumetric measurements predicted AD.

Conclusion. These results provide further support for the clinical use of MRI biomarkers for identification of prodromal $\mathrm{AD}$ among $\mathrm{MCl}$-patients. Assessment of hippocampal volume together with a delayed recall test may be sufficient for shortterm prediction of $A D$ in subjects with $\mathrm{MCl}$. 


\section{INTRODUCTION}

Due to the aging population, the prevalence and concomitant costs of neurodegenerative diseases including Alzheimer's Disease (AD) are expected to increase dramatically [1]. Before entering the phase of dementia, subjects with $A D$ experience a period in which they have so called mild cognitive impairments $(\mathrm{MCl})$ without encountering interference in daily life activities. Identification of subjects with $\mathrm{MCl}$ at risk for $A D$ is crucial, as these subjects are useful candidates for drug trials which aim to slow down disease progression.

New criteria for Alzheimer's disease (AD) recommend the use of cerebrospinal fluid (CSF), magnetic resonance imaging (MRI) or positron-emission tomography (PET) biomarkers for the diagnosis of $A D$ in subjects with $\mathrm{MCl}[2,3]$. However, in non-academic settings evaluation of AD pathology by means of CSF and PET biomarkers is often not feasible. In these settings diagnosis is primarily based upon neuropsychological evaluation, anamnestic information and MRI measurements. Despite numerous studies evaluating MRI measurements as a candidate biomarker for $A D$, the added value of MRI measures over neuropsychological tests is not clear yet.

Neuropsychological tests that can best predict AD-type dementia in subjects with $\mathrm{MCl}$ are typically episodic memory tasks, such as immediate and delayed recall [4, 5]. However, according to some studies, assessing other cognitive domains such as categorical fluency [6], visuoconstruction [7] and tasks of executive functioning $[4,8]$ may also be useful in predicting AD-type dementia in MCl-patients. MRI is routinely used to exclude other diseases or other causes of dementia [9] and medial temporal lobe (MTL) atrophy as assessed on structural MRI has furthermore proven to be an effective clinical aid in the prediction of AD-type dementia in subjects with $\mathrm{MCl}[10,11]$.

Previous studies that examined the added value of MRI over neuropsychological tests are inconclusive in nature. In one study, hippocampal and entorhinal cortex volumes showed limited added value to memory and measures of functioning [12], whereas in other studies $[13,14]$ was found that a combination of both memory and MRI increased predictive accuracy over each measurement alone. In a study 
of Zhou et al. [15] was found that the combination of the ADAS-Cog with the CDRsum of boxes had a slightly better predictive accuracy than the ADAS-Cog with cortical thickness of the right temporal lobe (conversion rate $92.7 \%$ and $88.8 \%$ respectively). Another study found that neuropsychological tests and MRI were the most informative techniques (compared to Positron Emission Tomography (PET)), with $84 \%$ and $82 \%$ correct classifications [16].

The aim of the present study was to evaluate the added value of MRI over neuropsychological tests for the prediction of $A D$ in subjects with $\mathrm{MCl}$ in a memory clinic population. Atrophy of the MTL was assessed using an automated atlas-based hippocampal measurement (LEAP) $[11,17]$ and a qualitative visual rating scale (MTAscore) [18]. We also investigated whether hippocampal volume had predictive value for AD-type dementia in a group of subjects with a high score on the Mini-Mental State Examination (MMSE) or with a normal MTA score. Test sequence analyses were performed to determine the optimal order of cognitive tests and hippocampal volume for the prediction of AD-type dementia [19].

\section{MATERIALS AND METHODS}

\section{Subjects}

Subjects with $\mathrm{MCl}$ were selected from DESCRIPA (Development of Screening Guidelines and Clinical Criteria for Predementia AD) [20]. The VUmc center, one of the DESCRIPA centers, contributed an additional sample of subjects that were seen outside the DESCRIPA inclusion period. Inclusion criteria were: age 54 years or older and diagnosis of $\mathrm{MCl}$. Exclusion criteria were diagnosis of dementia at baseline or any somatic, psychiatric or neurological disorder (e.g. epilepsy) that might have caused the cognitive impairment [20]. For the present study, participants were selected from 9 of the 20 participating centers as only in those centers both neuropsychological data and MRI measurements were available. Of the 270 subjects with $\mathrm{MCl}$ in these sites, 204 had MRI data available and, 184 of those patients also had complete data for the neuropsychological tests (see below) and completed at least 1 follow-up. There were no differences between included and excluded subjects with respect to age, gender, educational level, hippocampal volume, and cognitive test scores, except for the MMSE-score (included subjects: 27.47 (2.08) vs. excluded subjects: 26.29 (2.78); $p<.001)$. 


\section{Clinical assessment and follow-up}

All participants underwent a standard diagnostic workup, including clinical history, medical and neurological examination, clinical chemistry, functional evaluation using the Clinical Dementia Rating scale (CDR) [21], the MMSE, and rating scales for depression and neuropsychiatric symptoms. Baseline diagnosis of $\mathrm{MCl}$ was made according to the criteria of Petersen et al. [22]. Cognitive impairment was defined as a z-score <-1.5 SD on any of the following tests: the learning measure or delayed recall of a word list learning test or equivalent memory test, the trail making test (TMT) part A, TMT part B, categorical fluency, Rey figure copy test or an equivalent test [23]. Outcome measure was conversion to AD-type dementia after 2 years. $A D$ diagnosis was made according to the DSM-IV [24] and NINCDS-ADRDA criteria, [25].

\section{Cognitive testing}

A neuropsychological battery was carried out in order to evaluate performance in several cognitive domains. In each center a primary test for verbal memory was chosen that was identical or at least similar to tests used in the other participating centers (memory: Rey Auditory Verbal Learning Test $(n=136)$, Consortium to Establish a Registry for Alzheimer's Disease (CERAD, $n=28$ ) or Buschke Selective Reminding Test ( $n=20)$; attention and executive functioning: all TMT-A and TMT-B $(n=184)$, categorical fluency animals $(n=123)$ or fluency animals, fruits or cartypes $(n=61)$; visuoconstruction: Rey Figure copy test $(n=136)$, CERAD copy figures $(n=28)$, Copy figures from the Mental Deterioration Battery $(n=20)$ ) [20]. Raw scores on neuropsychological tests were corrected for age, education, and sex, in accordance with locally collected or published normative data and expressed as z-scores, which were used for further analysis.

\section{MRI acquisition and analysis}

At each site, patients were scanned according to the routine MRI-protocol. Scanners and protocols at different sites varied but all scanning was performed at 1.0 or 1.5 Tesla. All scans included a three-dimensional T1 weighted gradient echo (3DT1) sequence and a fast fluid attenuated inversion recovery (FLAIR) sequence. Two measurements of MTL atrophy were included: an automatically measured hippocampal volume based on atlas registration (LEAP) [17] and a qualitative visual rating scale [18]. Elaborate descriptions of these MRI protocols and measurements 
are stated in a previous publication [11].

\section{APOE genotype}

APOE genotype was determined by polymerase chain reaction of genomic DNA extracted from EDTA anticoagulated blood in 154 subjects. Subjects were classified as $A P O E-\varepsilon 4$ carriers or non-carriers.

\section{Statistical analysis}

Statistical analyses were performed with IBM SPSS version 20.0 (Chicago, IL, USA) and statistical software package $R$ ( $R$ foundation, Vienna, Austria). Differences between groups were analyzed using an independent sample t-test for continuous variables and chi-square test for categorical variables. Area under the curve (AUC) for AD-type dementia after 2 years follow-up was calculated using a timedependent ROC curve in R [26]. Sensitivity, specificity, positive predictive value (PPV), negative predictive value (NPV) and odds ratio (OR) for conversion to ADtype dementia after 2 years were calculated using data-driven cut-points based on a time dependent ROC. Cut-points were determined by maximizing the Youden Index (sensitivity+specificity-1) for prediction of AD-type dementia at 2-years follow-up. Significance was set at $p<0.05$ and $95 \%$ confidence intervals $(\mathrm{Cl})$ were calculated. Data for each neuropsychological test and MRI-measurement were dichotomized into separate variables according to these cut-points. Univariate and multivariate logistic regression analyses were performed for dichotomized variables to assess whether a combination of markers was more predictive for AD than each of these measurements alone. Demographic variables and MMSE score were added in the first step, univariate significant cognitive test scores in the second step and hippocampal volume or MTA score in the third step. After each step we only retained statistically significant predictors in the model. Sensitivity and specificity values were calculated based on the results of the regression model. To assess whether the model improved after each step, the increase in $\chi 2$ was tested. A predicted value of 0.5 assumed presence of the disease.

\section{RESULTS}

\section{Subject characteristics}

From the total group of $\mathrm{MCl}$-patients included in this study $40 \%$ were non-amnestic 
$\mathrm{MCl}$ ( $74 \%$ single-domain and $26 \%$ multi-domain) and $60 \%$ amnestic $\mathrm{MCl}$ ( $45 \%$ singledomain and $55 \%$ multi-domain). Subject characteristics at baseline are shown in Table 1. Subjects who converted to AD-type dementia at 2-years follow up were older, had lower baseline scores on the MMSE, the immediate and free delayed recall, the TMT-B and categorical fluency task compared to those who did not progress, and were often carrier of the APOE-e4 allele. Baseline hippocampal volumes were lower and MTA scores higher in converters compared to non-converters.

Table 1. Subject characteristics.

\begin{tabular}{lll}
\hline & Non-converters & Converters \\
\hline $\mathrm{N}$ & 148 & 36 \\
Age & $69.4(7.9)$ & $72.9(6.8)^{* *}$ \\
Female (\%) & 57 & 60 \\
Years education & $9.1(4.1)$ & $9.7(4.3)$ \\
MMSE score & $27.7(2.1)$ & $26.6(1.9)^{* *}$ \\
Z-score wordlist (free delayed recall) & $-1.17(1.20)$ & $-1.91(0.82)^{* * *}$ \\
Z-score wordlist (immediate free & & \\
recall) & $-1.03(1.04)$ & $-1.42(0.85)^{*}$ \\
Z-score Fluency & $-0.96(0.91)$ & $-1.20(0.72)$ \\
Z-score TMT-A & $-1.09(1.56)$ & $-0.69(1.25)$ \\
Z-score TMT-B & $-1.45(1.96)$ & $-1.44(1.71)$ \\
Z-score Rey Complex Figure & $-0.08(1.23)$ & $-0.03(1.16)$ \\
ApoE-e4-carrier (\%) & 42 & $59 *$ \\
Hippocampal volume (mm3) & $6016(747)$ & $5291(656)^{* * *}$ \\
MTA-score & $2.5(1.5)$ & $3.5(1.5)^{* *}$ \\
Average FU (year) & $2.37(0.79)$ & $1.51(0.58)^{* * *}$ \\
Average time to AD (year) & - & $1.38(0.55)$ \\
\hline A & &
\end{tabular}

All volumetric measurements are corrected for intracranial volume. Values are mean (SD). Abbreviations: $\mathrm{MMSE}=$ Mini-Mental state examination; TMT: Trial-making test; ApoE = apolipoprotein E genotype; LEAP: learning embeddings for atlas propagation; MTA: medial temporal lobe atrophy; FU = follow-up; $A D=$ Alzheimer's disease.

$* p<.05, * * p<.01, * * * p<.001$ for differences between groups.

\section{Univariate predictive accuracy}

The AUC for prediction of AD-type dementia at 2 years follow-up was around 0.70 for the MRI measurements, the MMSE and the delayed recall score, while it was 
below 0.61 for the other neuropsychological measurements (0.52-0.61) (Table 2). When scores were dichotomised based on a cut-point that maximised the Youden index, the overall predictive accuracy (odds ratio) was highest for hippocampal volume $(O R=7.8)$, followed by $M T A-s c o r e ~(O R=4.1)$ and the free delayed recall test $(O R=3.6)$, the MMSE-score $(O R=2.9)$, the immediate free recall test $(O R=2.5)$, categorical fluency $(O R=2.3)$ and TMT-B $(O R=1.9)$. TMT-A and Rey complex figure test did not predict $A D$ conversion. When MRI-measurements were investigated for both hemispheres separately, it was found that the predictive accuracy was somewhat higher for the left hippocampus compared to the right hippocampus. Differences in odds ratio for left and right MTA-score were negligible (Table 2).

\section{Multivariate predictors}

\section{Neuropsychology}

When age, MMSE score and neuropsychological tests were entered together, only age and delayed recall score independently predicted AD conversion (Table 3 ).

\section{Neuropsychology combined with MRI-markers}

When age, MMSE score and delayed recall score were combined with MRI measurements, hippocampal volume (change in $\chi 2=16.57 ; \mathrm{df}=1 ; p<.001$ ) but not the MTA-score $(p=.06)$ increased the likelihood of conversion (Table 3$)$. Both left and right hippocampal volume increased predictive accuracy (left: $\chi 2=18.47 \mathrm{df}$ $=1 ; p<.001$; right: $\chi 2=8.90 \mathrm{df}=1 ; p<.001$ ) (Table 3). The increase of predictive accuracy was due to an increase in both sensitivity and specificity.

\section{Test sequence}

Figure 1 shows probability plots of AD-type dementia for different sequences of delayed recall test and hippocampal volume in which the change in probability of $A D$-conversion is visualized after adding the result of each individual test. If hippocampal volume was analyzed first, assessment of the delayed recall task increased predictive accuracy in subjects with a normal hippocampal volume ( $\mathrm{OR}=4.3, p=0.02$ ) but not in subjects with abnormal hippocampal volumes (OR=1.3, $p=0.7$ ) (Figure $1 \mathrm{~A}$ ). If the delayed recall task was administered first, assessment of hippocampal volume increased predictive accuracy for AD-type dementia both in subjects with a normal delayed recall score $(\mathrm{OR}=16.4, p<0.001)$ and in subjects with an abnormal delayed recall score $(\mathrm{OR}=5.0, p<0.001)$ (Figure $1 \mathrm{~B})$. 


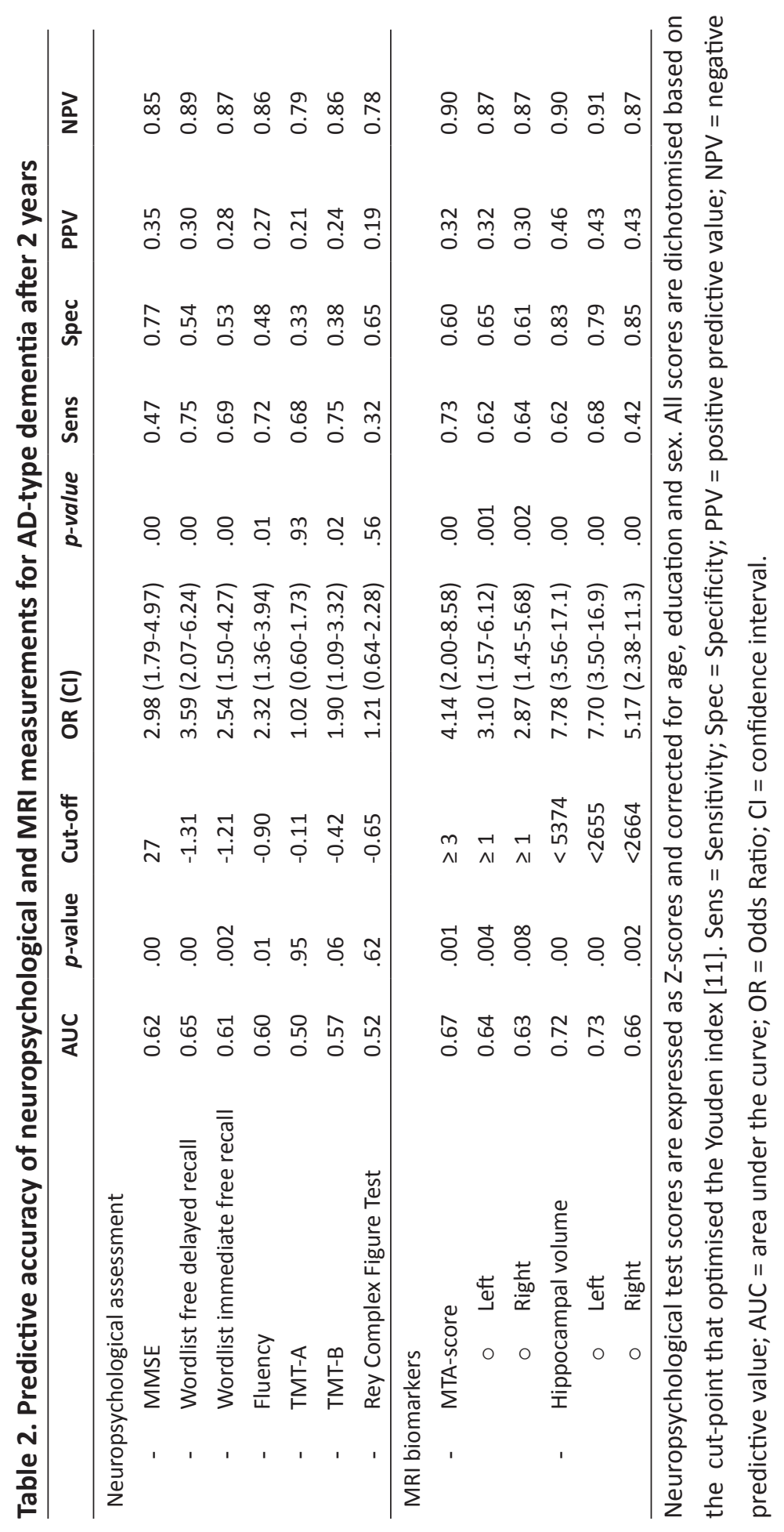




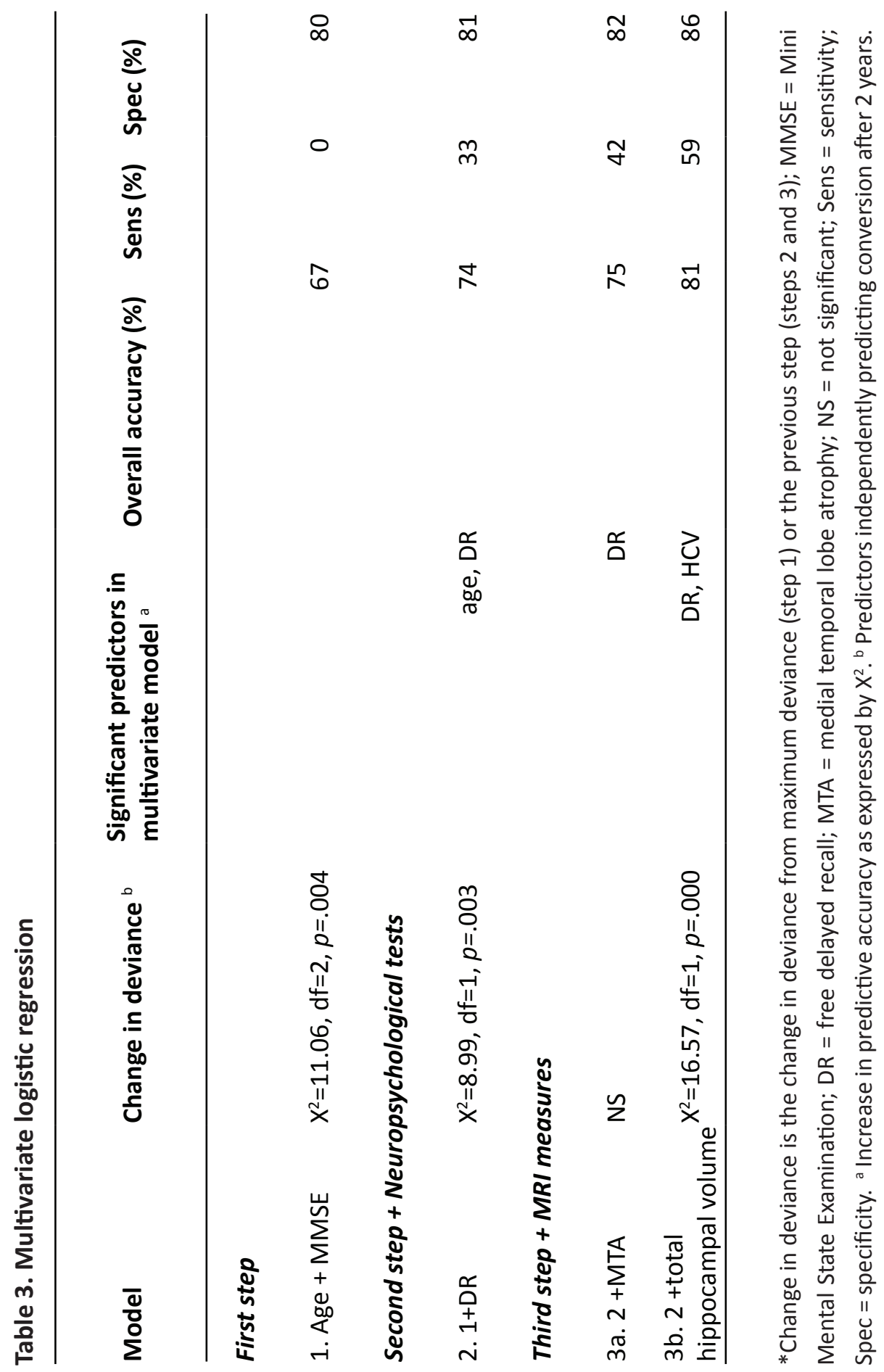

36 | Chapter 2: Added value of MRI-biomarkers 


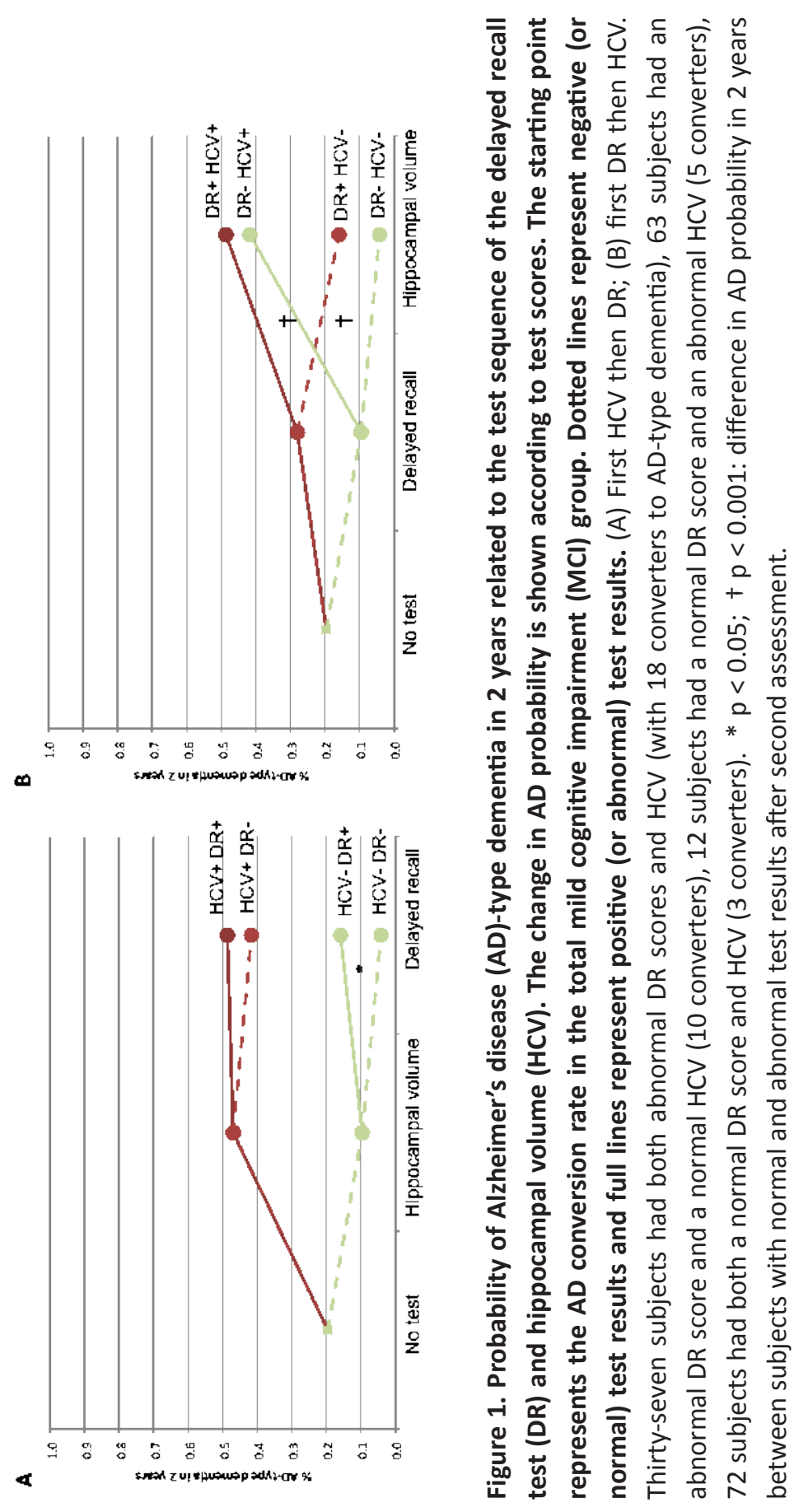

Chapter 2: Added value of MRI-biomarkers | 37 


\section{Predictive value in subjects with a a MMSE or MTA score above cut-off}

If subjects with an MMSE-score $>27$ were selected, ( $n=106$, of which 14 converted) both delayed recall and MRI measurements at baseline predicted AD-type dementia at follow-up (Table 5). In subjects with a normal MTA-score (summed left and right score $<3$ ) ( $n=105$, of which 12 converted), hippocampal volume but not delayed recall or MMSE score predicted progression (Table 5).

Table 4. Univariate predictive accuracy in cases with MMSE $>27$ or MTA-score $<3$

\begin{tabular}{llllll}
\hline Measure & $\mathrm{OR}(\mathrm{Cl})$ & $p$ & $\mathrm{X}^{2}$ & $\mathrm{df}$ & $p$ \\
\hline A Predictive &
\end{tabular}

A. Predictive accuracy MMSE $>27 *$

$\begin{array}{llllll}\text { DR } & 9.17(1.94-43.40) & 0.005 & 11.14 & 1 & 0.001\end{array}$

$\begin{array}{llllll}\text { Hippocampal volume } & 5.87(2.40-14.30) & 0 & 14.9 & 1 & 0\end{array}$

\begin{tabular}{llllll} 
MTA & $2.71(1.16-6.34)$ & 0.02 & 5.44 & 1 & 0.02 \\
\hline
\end{tabular}

\section{B. Predictive accuracy MTA $<3 * *$}

\begin{tabular}{lrrrrr}
\hline MMSE-score & $2.45(0.71-8.52)$ & 0.16 & 1.88 & 1 & 0.17 \\
DR & $2.04(0.56-7.46)$ & 0.28 & 1.21 & 1 & 0.27 \\
Hippocampal volume & $6.53(2.84-14.98)$ & 0 & 19.59 & 1 & 0
\end{tabular}

Data-selection based on A. MMSE $>27$ or $\mathrm{B}$. MTA-score below 3. OR = odds ratio; $\mathrm{Cl}$ = confidence interval; $\mathrm{df}=$ degrees of freedom; $\mathrm{DR}=$ free delayed recall score; MMSE = mini-mental state examination score. *Baseline MMSE $>27$ and AD at follow-up ( $n=14)$;

**Baseline MTA $<3$ and AD at follow-up ( $n=12)$.

\section{DISCUSSION}

Our main finding is that hippocampal volume but not MTA-score increases the predictive accuracy of the delayed recall score for the prediction of AD-type dementia after 2 years. Predictive accuracy was increased both in subjects with normal and abnormal delayed recall scores at baseline. In the subgroup of subjects with an MMSE score $>27$, both delayed recall and MRI measurements at baseline were predictors of conversion at follow-up. In the group of subjects with a normal MTA-score (<3), only volumetric measurements predicted AD. 


\section{Neuropsychological predictors}

In line with previous studies, delayed recall appeared the best neuropsychological predictor [27-31]. We found that categorical fluency and TMT-B had a moderately high predictive accuracy for AD. Previous studies also found that the TMT-B task predicted $A D$ in amnestic $\mathrm{MCl}[32,33]$ and that categorical fluency was the second most sensitive test in the detection of cognitive changes due to prodromal AD [34]. Important to note however is that other disorders may cause low performance on delayed recall tests. Disorders which can impair attentional processes (e.g. depression, anxiety) may result in poor registration and encoding of information [35] and disorders of executive functioning may moreover result in retrieval problems [36]. The same pattern can be identified in our data, whereas a cut-point of -1.31 (Z-score) on the delayed recall task shows a high sensitivity together with a low specificity. Cued recall techniques on the other hand are thought to minimize the effect of attentional deficits and thus be more specific for $\operatorname{AD}[5,35,37]$.

\section{MRI predictors}

In line with previous studies, it was found that visual rating scales show lower predictive accuracy compared to volumetric measurements [11]. The present study furthermore confirmed that in a group of subjects with a normal MTA-score $(<3)$, volumetric measurements still independently predicted $A D$-conversion, indicating that hippocampal volume is more sensitive for $A D$ than a visual rating scale. Our finding of left>right is in line with one study investigating a large ADNI cohort stating that left hippocampal volume at baseline was the best single predictor of AD-conversion [38]. Another study in cognitively normal subjects reported that left hippocampal volume was a predictor for verbal memory function [39].

\section{Neuropsychology and MRI-measurements}

The present study found that hippocampal volume increased predictive accuracy relative to a model with only neuropsychological information. Furthermore, test sequence analysis found that assessment of hippocampal volume increased predictive accuracy both in subjects with a normal and abnormal delayed recall score. In line with these findings, a recent study found the highest predictive accuracy for a model that combined a delayed recall test, functional measures and 
hippocampal and entorhinal cortex volumes with age and MMSE-score [12]. Unlike hippocampal volume, visual MTA-ratings did not increase the predictive accuracy for conversion in a model with the delayed recall task. In line with the added value of hippocampal volume in subjects with normal memory scores, hippocampal volume also predicted conversion in subjects with a normal MMSE-score. These findings indicate the necessity of adding volumetric measurements of the hippocampus to standard neuropsychological investigation in order to increase the certainty that the $\mathrm{MCl}$ syndrome is due to AD-pathology.

\section{Limitations}

We determined cut-offs within a study population that also included subjects from the present analyses. This could have led to an overestimation of the predictive accuracy. However, it is unlikely that it influenced our findings with respect to the differences in predictive accuracy between measurements, as the same method was used to define the cut-pointforeachmeasurement. The diagnosis of ADatfollow-up was notvalidated neuropathologically which may have possibly led to a misclassification of some cases. Follow-up data with AD diagnosis for all subjects was only available for relatively short follow-up intervals ( 2 years). A recent meta-analysis found that predictive accuracy for MTL atrophy decreased when follow-up was longer [40]. Still, shortterm prognosis may be important for selection of subjects for trials. Recent studies point out the importance of cued recall tasks as specific marker for AD-conversion $[5,29,35,41,42]$. One could suggest using free delayed recall tasks for diagnosing $\mathrm{MCl}$, and cued recall tasks to predict which $\mathrm{MCl}$ patients are likely to convert, and thus represent a group of prodromal AD-patients [35, 42]. A recent study found biological evidence for the role of cued recall as neuropsychological marker for early AD [41]. Unfortunately, in our study no cued recall task was available. Candidate tests which can be considered in future studies are the Visual Association Test (VAT) [43] and the Memory Impairment Screen (MIS) [44]. A recent study found that the MISplus showed highest predictive accuracy values in predicting future ADconversion in subjects with $\mathrm{MCl}$ [5].Scanners with different field strengths (1T and 1.5T) were used, reflecting real-life situations in which scanners and magnetic field strengths do vary. A previous study showed that it is unlikely that a small difference in field strength has a major impact on volumetric measurements [11]. It can be 
considered a strength that a population from a memory clinic setting was used for this study. Conversely, findings may not be readily generalised to community populations.

\section{Implications for clinical practice}

Our findings indicate that a combination of neuropsychological data, especially the administration of delayed recall tasks, together with hippocampal volume is preferred. Sensitivity and specificity increases when hippocampal volume is added to a model which only includes neuropsychological data. In addition, structural MRI assessment is useful to rule out specific causes of cognitive impairment (e.g. stroke, tumor) [12]. The added value of performing a visual rating scale on top of neuropsychological testing is however limited in terms of sensitivity and specificity (no increase in overall predictive accuracy).Although a combination of both neuropsychology and volumetry is preferred, predictive accuracy values of hippocampal assessment alone are comparable. Since the likelihood to detect MCl patients at risk for ADconversion is highest for volumetric measurements, volumetric assessment of MTL atrophy together with a delayed recall task may be sufficient for the diagnostic investigation of $\mathrm{MCl}$-patients. In addition, one could consider adding elaborate neuropsychological investigation in subjects with normal hippocampal volumes.

Assessing disease progression by means of episodic memory tests (delayed recall) and MMSE-score is an alternative at the cost of a lower sensitivity and specificity. For example, subject may progress to AD but still perform relatively well on cognitive tests (low sensitivity). Moreover, not all subjects with memory impairment progress to $A D$ (low specificity). Free recall is moreover dependent on retrieval mechanisms, motivational and attentional processes. In line with recent criteria, however, one should additionally assess whether free recall deficits can be normalized with cueing. Failure to recall words after the presence of cues is indicative of prodromal $A D$ [3]. When applying a more extensive neuropsychological test battery one should consider including MMSE-score, a verbal wordlist test (including immediate and free delayed recall), a fluency test and a test for executive functioning (e.g. TMT-B task), which are all shown to be independent predictors of AD-conversion in subjects with $\mathrm{MCl}$. 
We previously showed that CSF markers further improved predictive accuracy over hippocampal atrophy by increasing the sensitivity [21]. Combined use of both CSF and imaging biomarkers is highly accurate for either early diagnosis or exclusion of $A D$ in patients with $\mathrm{MCl}[21,32,39-41]$. Future studies could add both MRI and CSF measurements to neurospychological data in order to further increase predictive accuracy.

\section{CONCLUSION}

The present study found that volumetric measurements are the best predictors of $\mathrm{AD}$-conversion after 2 years in a group of $\mathrm{MCl}$-subjects. Assessment of hippocampal volume together with a delayed recall test may be sufficient for short-term prediction of $A D$ in subjects with $\mathrm{MCl}$. 


\section{REFERENCES}

1. Peters KR, Lynn Beattie B, Feldman HH, Illes J. A conceptual framework and ethics analysis for prevention trials of Alzheimer Disease. Progress in Neurobiology 2013.

2. Albert MS, DeKosky ST, Dickson D, et al. The diagnosis of mild cognitive impairment due to Alzheimer's disease: Recommendations from the National Institute on Aging-Alzheimer's Association workgroups on diagnostic guidelines for Alzheimer's disease. Alzheimer's \& dementia : the journal of the Alzheimer's Association 2011;7:270-279.

3. Dubois B, Feldman HH, Jacova C, et al. Revising the definition of Alzheimer's disease: a new lexicon. The Lancet Neurology 2010;9:1118-1127.

4. Dickerson BC, Sperling RA, Hyman BT, Albert MS, Blacker D. Clinical prediction of alzheimer disease dementia across the spectrum of mild cognitive impairment. Archives of General Psychiatry 2007;64:1443-1450.

5. Dierckx E, Engelborghs $S$, De Raedt R, et al. Verbal cued recall as a predictor of conversion to Alzheimer's disease in Mild Cognitive Impairment. International Journal of Geriatric Psychiatry 2009;24:1094-1100.

6. Riley KP, Jicha GA, Davis D, et al. Prediction of Preclinical Alzheimer's Disease: Longitudinal Rates of Change in Cognition. Journal of Alzheimer's Disease 2011;25:707-717.

7. Artero S, Tierney MC, Touchon J, Ritchie K. Prediction of transition from cognitive impairment to senile dementia: a prospective, longitudinal study. Acta Psychiatrica Scandinavica 2003;107:390-393.

8. Albert MS, Moss MB, Tanzi R, Jones K. Preclinical prediction of AD using neuropsychological tests. J Int Neuropsychol Soc. 2001;7:631-639.

9. Benzinger TLS. Radiologic approach to Alzheimer's disease and other dementias: The emerging role of diffusion tensor magnetic resonance imaging. Applied Radiology 2005;34:25-33.

10. Bouwman FH, Schoonenboom SN, van der Flier WM, et al. CSF biomarkers and medial temporal lobe atrophy predict dementia in mild cognitive impairment. Neurobiol Aging 2007;28:1070-1074.

11. Clerx L, van Rossum I, Burns L, et al. Measurements of medial temporal lobe atrophy in the prediction of Alzheimer's Disease in subjects with $\mathrm{MCl}$. Neurobiology of Aging 2013.

12. Devanand DP, Liu X, Brown PJ, Huey ED, Stern Y, Pelton GH. A Two-Study Comparison of Clinical and MRI Markers of Transition from Mild Cognitive Impairment to Alzheimer's Disease. Int J Alzheimers Dis. 2012;2012.

13. Visser PJ, Scheltens P, Verhey FRJ, et al. Medial Temporal lobe atrophy and memory dysfunction as predictors for dementia in subjects with mild cognitive impairment. Journal of Neurology 1999;246:477-485.

14. Visser PJ, Verhey FRJ, Hofman PAM, Jolles J. Medial temporal lobe atrophy predicts Alzheimer's disease in patients with minor cognitive impairment. J 
Neurol Neurosurg Psychiatry 2002;72:491-497.

15. Zhou B, Nakatani E, Teramukai S, Nagai Y, Fukushima M. Risk Classification in Mild Cognitive Impairment Patients for Developing Alzheimer's Disease. Journal of Alzheimer's Disease 2012;30:367-375.

16. Schmand B, Eikelenboom P, van Gool WA, the Alzheimer's Disease Neuroimaging I. Value of Neuropsychological Tests, Neuroimaging, and Biomarkers for Diagnosing Alzheimer's Disease in Younger and Older Age Cohorts. Journal of the American Geriatrics Society 2011;59:1705-1710.

17. Wolz R, Aljabar P, Hajnal JV, Hammers A, Rueckert D. LEAP: Learning embeddings for atlas propagation. Neurolmage 2010;49:1316-1325.

18. Scheltens P, Leys D, Barkhof F, et al. Atrophy of medial temporal lobes on MRI in "probable" Alzheimer's disease and normal ageing: diagnostic value and neuropsychological correlates. J Neurol Neurosurg Psychiatry 1992;55:967972.

19. Severens JL, Sonke G, Laheij RJF, Verbeek ALM, De Vries Robbé PF. Efficient diagnostic test sequence: Applications of the probability-modifying plot. Journal of clinical epidemiology 2001;54:1228-1237.

20. Visser PJ, Verhey FRJ, Boada M, et al. Development of Screening Guidelines and Clinical Criteria for Predementia Alzheimer,Äôs Disease. Neuroepidemiology 2008;30:254-265.

21. Morris JC. The Clinical Dementia Rating (CDR): current version and scoring rules. Neurology 1993;43:2412-2414.

22. Petersen RC, Smith GE, Waring SC, Ivnik RJ, Tangalos EG, Kokmen E. Mild Cognitive Impairment: Clinical Characterization and Outcome. Archives of Neurology 1999;56:303-308.

23. Vos S, van Rossum I, Burns $L$, et al. Test sequence of CSF and MRI biomarkers for prediction of AD in subjects with $\mathrm{MCl}$. Neurobiology of Aging 2012.

24. APA APA. Diagnostic and Statistical Manual of Mental Disorders, 4th edition. Washington, D.C., 1994.

25. McKhann G, Drachman D, Folstein M, Katzman R, Price D, Stadlan EM. Clinical diagnosis of Alzheimer's disease: report of the NINCDS-ADRDA Work Group under the auspices of Department of Health and Human Services Task Force on Alzheimer's Disease. Neurology 1984;34:939-944.

26. Heagerty PJ, Lumley T, Pepe MS. Time-Dependent ROC Curves for Censored Survival Data and a Diagnostic Marker. Biometrics 2000;56:337-344.

27. Devanand DP, Liu X, Tabert $\mathrm{MH}$, et al. Combining Early Markers Strongly Predicts Conversion from Mild Cognitive Impairment to Alzheimer's Disease. Biological Psychiatry 2008;64:871-879.

28. Fleisher AS, Sowell BB, Taylor C, et al. Clinical predictors of progression to Alzheimer disease in amnestic mild cognitive impairment. Neurology 2007;68:1588-1595.

29. Sarazin M, Berr C, De Rotrou J, et al. Amnestic syndrome of the medial

44 | Chapter 2: Added value of MRI-biomarkers 
temporal type identifies prodromal AD: A longitudinal study. Neurology 2007;69:1859-1867.

30. Schmand B, Eikelenboom P, van Gool WA. Value of Diagnostic tests to Predict Conversion to Alzheimer's Disease in Young and Old Patients with Amnestic Mild Cognitive Impairment. Journal of Alzheimer's Disease 2012;29:641-648.

31. Varela-Nallar L, Aranguiz FC, Abbott AC, Slater PG, Inestrosa NC. Adult hippocampal neurogenesis in aging and Alzheimer's disease. Birth Defects Research Part C: Embryo Today: Reviews 2010;90:284-296.

32. Arbuthnott K, Frank J. Trail Making Test, Part B as a Measure of Executive Control: Validation Using a Set-Switching Paradigm. Journal of Clinical and Experimental Neuropsychology 2000;22:518-528.

33. Ewers M, Walsh C, Trojanowski JQ, et al. Prediction of conversion from mild cognitive impairment to Alzheimer's disease dementia based upon biomarkers and neuropsychological test performance. Neurobiology of Aging 2012;33:1203-1214.e1202.

34. Mura T, Proust-Lima $\mathrm{C}$, Jacqmin-Gadda $\mathrm{H}$, et al. Measuring cognitive change in subjects with prodromal Alzheimer's disease. Journal of Neurology, Neurosurgery \& Psychiatry 2013.

35. Dierckx E, Engelborghs S, De Raedt R, De Deyn PP, Ponjaert-Kristoffersen I. Differentiation between mild cognitive impairment, Alzheimer's disease and depression by means of cued recall. Psychological Medicine 2007;37:747755.

36. Fossati P, Harvey P-O, Le Bastard G, Ergis A-M, Jouvent R, Allilaire J-Fo. Verbal memory performance of patients with a first depressive episode and patients with unipolar and bipolar recurrent depression. Journal of Psychiatric Research 2004;38:137-144.

37. Ivanoiu A, Adam S, Linden $\mathrm{M}$, et al. Memory evaluation with a new cued recall test in patients with mild cognitive impairment and Alzheimer, Äôs disease. Journal of Neurology 2005;252:47-55.

38. Risacher SL, Saykin AJ, West JD, et al. Baseline MRI Predictors of Conversion from MCI to Probable AD in the ADNI Cohort. Curr Alzheimer Res 2009;6:347361.

39. Ystad M, Lundervold A, Wehling E, et al. Hippocampal volumes are important predictors for memory function in elderly women. BMC Medical Imaging 2009;9:17.

40. Schmand B, Huizenga HM, van Gool WA. Meta-analysis of CSF and MRI biomarkers for detecting preclinical Alzheimer's disease. Psychological Medicine 2010;40:135-145.

41. Wagner M, Wolf S, Reischies FM, et al. Biomarker validation of a cued recall memory deficit in prodromal Alzheimer disease. Neurology 2012.

42. Dubois B, Feldman HH, Jacova C, et al. Research criteria for the diagnosis of Alzheimer's disease: revising the NINCDS-ADRDA criteria. The Lancet 
Neurology 2007;6:734-746.

43. Lindeboom J, Schmand B, Tulner L, Walstra G, Jonker C. Visual association test to detect early dementia of the Alzheimer type. Journal of Neurology, Neurosurgery \& Psychiatry 2002;73:126-133.

44. Buschke $H$, Kuslansky $G$, Katz $M$, et al. Screening for dementia with the Memory Impairment Screen. Neurology 1999;52:231-231. 



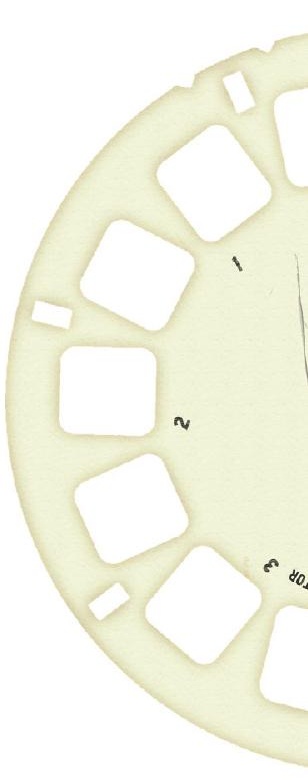




\section{Chapter 3 \\ Measurements of medial temporal lobe atrophy for prediction of $A D$ in subjects with $\mathrm{MCl}$}

Neurobiology of Aging. 2013 Aug;34(8):2003-13

Lies Clerx, Ineke A.van Rossum, Leah Burns, Dirk L. Knol, Philip Schelten, Frans Verhey, Pauline Aalten, Pablo Lapuerto, Laura van de Pol, Ronald van Schijndel, Remko de Jong, Frederik Barkhof, Robin Wolz, Daniel Rueckert, Martina Bocchetta, Magdalini Tsolaki, Flavio Nobili, Lars-Olaf Wahlund, Lennart Minthon, Lutz Frölich, Harald Hampel, Hilkka Soininen \& Pieter Jelle Visser. 


\section{ABSTRACT}

Our aim was to compare the predictive accuracy of four different medial temporal lobe measurements for Alzheimer's disease (AD) in subjects with mild cognitive impairment $(\mathrm{MCl})$. Manual hippocampal measurement, automated atlas based hippocampal measurement (LEAP), a visual rating scale (MTA-score), and lateral ventricle measurement were compared. Predictive accuracy for AD 2 years after baseline was assessed by ROC analyses with area under the curve (AUC) as outcome. Annual cognitive decline was assessed by slope analyses up to 5 years after baseline. Correlations with biomarkers in cerebrospinal fluid (CSF) were investigated. Subjects with $\mathrm{MCl}$ were selected from the DESCRIPA multicenter study $(\mathrm{n}=156)$ and the singlecenter VU medical center ( $n=172)$. At follow-up, AUC was highest for LEAP (0.71) and manual hippocampal measurement (0.71), while lower for MTA-score (0.65) and lateral ventricle (0.60). Slope analysis yielded similar results. Hippocampal measurements correlated with CSF total tau (t-tau) and phosphorylated tau (p-tau), not with beta-amyloid $(A \beta) 1-42$. MTA-score and lateral ventricle volume correlated with CSF A $\beta 1-42$. We can conclude that volumetric hippocampal measurements are the best predictors of $A D$ conversion in subjects with $\mathrm{MCl}$. 


\section{INTRODUCTION}

Alzheimer's disease (AD) is the leading cause of dementia in the elderly affecting more than 27 million people worldwide. Early detection of $A D$ may prevent irreversible damage by enabling preventative treatment [1, 2]. A primary focus of research in this area is identifying which biomarkers are clinically useful for the early diagnosis of $A D$.

Medial temporal lobe (MTL) atrophy as assessed on structural MRI has proven to be an effective clinical aid in the early diagnosis of $A D$ [3], and this method predicts $A D$ in subjects with mild cognitive impairment (MCl) [3-7]. There are several ways to determine the degree of MTL atrophy in the brain including manual delineation, (semi-) automated techniques to measure hippocampal volume, qualitative ratings of MTL atrophy (MTA-score), and assessment of lateral ventricular volume [8-18]. Each of these methods has its strengths and limitations.

Manual volumetry is considered the gold standard [19-21] but is time consuming, limiting routine clinical or large-scale research use. Automated measurements are quick and widely applicable, but may be susceptible to scanner and scan protocol variability. Volumetric measurements of the lateral ventricle require a minimum of rater time with robust automatic segmentations but show a lot of variability and asymmetry between subjects [14]. Qualitative ratings are quick to perform but sensitive to interrater variability and show lower accuracy rates compared to volumetric analysis $[22,23]$. Visual rating scales are furthermore insensitive to detect atrophy progression over time [24].

A number of studies have examined differences between various techniques to measure atrophy of the MTL, mostly comparing manual with automated hippocampal volumetry [25-27] or volumetric hippocampal measurements to a visual rating scale $[15,24,28-30]$. These studies typically evaluate the diagnostic accuracy of different MRI techniques by comparing $A D$ patients with healthy control subjects. Most studies found that manual hippocampal measurement shows similar performance as automated hippocampal segmentation [25, 31]. However, the performance of automated techniques may be less precise when applied in $A D$ patients suffering from moderate to severe brain atrophy and/or white matter 
hyperintensities which may lead to false allocations of gray matter, white matter or cerebrospinal fluid (CSF) [32-34]. One author stated that visual rating of MTL atrophy is a quick and clinically useful technique for differentiating AD from controls and is quicker and more accurate than volumetry [29].

To our knowledge, no study has compared the diagnostic performance of manual and atlas-based hippocampal segmentation, lateral ventricle volume and a qualitative rating. Moreover, no comparative studies have been performed on the predictive accuracy of these different methods to predict $A D$ in subjects with $\mathrm{MCl}$, their relation with CSF biomarkers of $A D$, and the of multi-center settings on diagnostic performance.

The aim of the present longitudinal study was to compare the predictive accuracy of four different MTL measurements for the progression to AD-type dementia in patients with $\mathrm{MCl}$ over a 2-year follow up period. Atrophy of the MTL was assessed using manual measurement of the hippocampus, automatically measured hippocampal volume based on atlas registration (LEAP), volumetric measurement of the expansion of the lateral ventricle and a largely used qualitative rating scale. Since subjects may convert at a later point in time, slope analyses were additionally performed with annual cognitive decline up to 5 years as outcome measure. The correlation of MTL measures with AD biomarkers in CSF was also investigated and the predictive accuracy was tested both in a multicenter study with different scan protocols and in a single-center study.

\section{MATERIALS AND METHODS}

\section{Subjects}

We selected participants with $\mathrm{MCl}$ from the DESCRIPA (Development of Screening Guidelines and Clinical Criteria for Predementia AD) study and the Alzheimer Center of the VU University Medical center (VUmc) in Amsterdam. DESCRIPA is a multicenter prospective cohort study from the European Alzheimer's Disease Consortium (EADC) aimed at developing clinical criteria and screening guidelines for predementia AD [35]. For this study participants were selected from 9 of the 20 participating centers where MRI scanning was performed as part of clinical practice or as research protocol. The VUmc cohort in the present study included the VUmc subjects enrolled in the DESCRIPA study and an additional sample of subjects that

52 | Chapter 3: MTL measurements for AD prediction in $\mathrm{MCl}$ 
were seen outside the DESCRIPA inclusion period. (Appendix S1).

Inclusion criteria for both cohorts were: age 54 years or older, diagnosis of $\mathrm{MCl}$ and availability of results for each MRI measure and outcome at follow-up. Exclusion criteria were diagnosis of dementia at baseline or any somatic, psychiatric or neurological disorder (e.g. epilepsy) that might have caused the cognitive impairment [35]. At baseline scans were available for 456 subjects. Visually rated MTL atrophy was available for all subjects. Of these, 54 had no FU data and were excluded. Of the remaining 402 subjects, scans were not available in digital format for 21 subjects. Of the remaining 381 scans, manual segmentation of the hippocampus could be performed on 341 scans (reason missings: technical problem volumetric measurement $[n=5]$, technical problem automated intracranial volume estimation $[n=25]$ and logistics [ $n=10]$ ), LEAP based volumetry on 357 scans (reason missings: technical problem volumetric measurement $[n=11]$ and logistics [ $n=13]$ ), and lateral ventricle volumetry on 335 scans (reason missings: technical problem volumetric measurement [ $n=37]$, and logistics [n=9]). Data for all four medial temporal lobe measurements was available for 328 subjects, 156 from DESCRIPA and 172 from VUmc. There were no differences between included and excluded subjects with respect to age, gender, educational level and cognitive test results.

\section{Clinical and cognitive assessment}

All participants underwent a standard diagnostic workup, including clinical history, medical and neurological examination, clinical chemistry, functional evaluation using the Clinical Dementia Rating scale (CDR) [36], the Mini-Mental State Examination (MMSE), and rating scales for depression and neuropsychiatric symptoms. A neuropsychological battery was performed to evaluate performance in several cognitive domains. In each center a primary test for verbal memory, language, attention, executive functioning, and visuoconstruction was chosen that was identical or similar to tests used in other centers [35]. Raw scores on neuropsychological tests were corrected for age, education, and sex, in accordance with locally collected or published normative data and expressed as z-scores, which were used for further analysis. Baseline diagnosis of $\mathrm{MCl}$ was made according to the criteria of Petersen et al. [37, 38]. MCl was defined as a z-score <-1.5 SD on any of the following tests: the learning measure or delayed recall of a word list learning test or equivalent memory test, the trail making test (TMT) part A, TMT 
part B, verbal fluency, Rey figure copy test or an equivalent test [39]. We calculated a composite score as the average $z$-score of the 6 tests if scores were available for at least 3 tests [40].

Follow-up was conducted annually for up to 5 years. The primary outcome measure was conversion to AD-type dementia after 2 years. AD diagnosis was made according to the DSM-IV [41] and NINCDS-ADRDA criteria, [42]. Secondary outcome measures were annual cognitive decline on the MMSE and the cognitive composite score.

\section{MRI acquisition and image analysis}

Scan protocol

At each site, patients were scanned according to the routine MRI-protocol. Scanners and protocols at different sites varied but all scanning was performed at 1.0 or 1.5 Tesla (Appendix S1). All scans included a three-dimensional T1 weighted gradient echo (3DT1) sequence and a fast fluid attenuated inversion recovery (FLAIR) sequence.

\section{MRI-measurements}

MTL atrophy was assessed using manual measurement of the hippocampus, automatically measured hippocampal volume using multi-atlas segmentation (LEAP), volumetric measurement of the expansion of the lateral ventricle and a qualitative rating. All volumetric measurements were corrected for intracranial volume (ICV). The LEAP measurement was ICV corrected by means of a scaling factor from $\mathrm{MNI}$ (Montreal Neurological Institute), the manual hippocampal measurement and the lateral ventricle measurement were ICV corrected by means of a scaling factor derived from FSL software. The total rating time of each method can be found in Supplementary Table S1. Rater time needed for analysis was lowest for LEAP hippocampal measurement (4 minutes) followed by the qualitative rating (5 minutes). Rater time was 30 minutes for lateral ventricle measurement and 150 minutes for manual hippocampal volumetry.

For the manual segmentation of the hippocampus, the baseline 3DT1-weighted volume sequence was reformatted in 2-mm slices (in-plane resolution: $1 \times 1 \mathrm{~mm}$ ) and oriented perpendicular to the long axis of the left hippocampus [21]. Regions of interest (ROIs) of the hippocampus were constructed by manual delineation of hippocampal borders on both sides on the reformatted slices, using the in-house-

54 | Chapter 3: MTL measurements for AD prediction in $\mathrm{MCl}$ 
developed software package Show_Images 3.7.0 (VU University Medical Center, Amsterdam, The Netherlands). Delineation of the hippocampus was performed using previously described criteria [43-45] by 3 trained technicians (coefficients of variation: interrater $<8 \%$, intrarater $<5 \%$ ) blinded to diagnosis. ROIs included the dentate gyrus, cornu ammonis, subiculum, fimbria, and alveus. Baseline hippocampal volume was calculated by multiplying the total area of all ROIs of each hippocampus by slice thickness. Baseline hippocampal volumes were adjusted for intracranial volume (ICV), using the scaling factor derived from SIENAX (FSL, FMRIB, Oxford, UK) [46].

Automated hippocampal volumetry was performed using the Learning embeddings for atlas propagation (LEAP) method [17]. In this method, multi-atlas registration is applied to a cohort of brain images after representing the whole population together with an initial set of atlases. The initial set is propagated to a number of unlabeled images in their local neighbourhood which are used to label them. Images labeled in this way become atlases themselves and are, consequently, further propagated throughout the whole dataset. In this way, each image is labeled using a number of atlases in its close vicinity, which has been shown to perform more robust on diverse datasets than other multi-atlas registration techniques [17]. A brief visual inspection of the segmented hippocampi was performed in order to identify clear failures of the automated method. Except for technical failures listed below, no subjects were excluded after this inspection and no manual correction was performed.

Measurement of the lateral ventricle was executed with an extension of SIENAX $[47,48]$, part of FSL [49]. SIENAX starts by extracting brain and skull images from the single whole-head input data [50]. The brain image is then affine-registered to MNI152 space $[51,52]$ using the skull image to determine the registration scaling. This is primarily in order to obtain the volumetric scaling factor, to be used as a normalisation for head size. Next, tissue-type segmentation is carried out [53] in order to calculate total volume of brain tissue (including separate estimates of volumes of grey matter, white matter, peripheral grey matter and ventricular CSF). After the tissue segmentation, a registered mask is used to exclude the CSF on the outer side of the brain. The resulting ventricular structure is manual edited when the segmentation or the mask did not take the whole ventricle into account or did contain CSF pixels not belonging to the ventricles (CSF found outside the ventricles). The visual rating of MTL atrophy was performed using a qualitative scale [15]. Rating was performed on coronal T1-weighted images using a 5-point visual scale (MTA- 
scores), ranging from 0 (no atrophy) to 4 (severe atrophy) based on the height of the hippocampal formation and the surrounding CSF spaces. In the analysis, the sum of both sides (left and right) was used. All visual rating was performed at VUmc by a number of trained raters. Scans from VUmc were rated by a group of 3 raters supervised by a neuroradiologist (intrarater weighted Cohen $\mathrm{K}>0.80$; interrater weighted Cohen $\kappa>0.80$ ) [54]. The Descripa scans were rated by a single rater from VUmc (intrarater weighted Cohen $\kappa=0.68$ ) [45].

\section{CSF analyses}

CSF was collected by lumbar puncture and levels of beta amyloid $(A \beta) 1-42$, total tau ( $t$-tau) and phosphorylated tau ( $p$-tau) in CSF were measured using commercially available sandwich ELISAs (Innotest $\beta$-amyloid 1-42; Innotest hTAU-Ag; Innogenetics, Ghent, Belgium), specially constructed to measure $A \beta 1-42$ and t-tau $[55,56]$ by experienced technicians at the lab in Gothenburg for the DESCRIPA cohort and in Amsterdam for the VUmc cohort. CSF was available for 147 subjects. We corrected for interlaboratory ELISA differences by analyzing 33 samples at both labs and we adjusted VUmc values to those of DESCRIPA using the following equating formula: Gothenborg= average Gothenborg + (SD Gothenborg/SD VUmc)*(VUmc - average VUmc) [57]. Abnormal values for CSF measures were a concentration $\leq 550 \mathrm{pg} / \mathrm{mL}$ for $A \beta 1-42,>52 \mathrm{pg} / \mathrm{mL}$ for $\mathrm{p}$-tau and $>375 \mathrm{pg} / \mathrm{mL}$ for t-tau [58].

\section{APOE genotype}

APOE genotype was determined by polymerase chain reaction of genomic DNA extracted from EDTA anticoagulated blood in 262 subjects. Subjects were classified as APOE- $\varepsilon 4$ carriers or non-carriers.

\section{Statistical analysis}

Statistical analysis was performed with IBM SPSS version 19 (Chicago, IL, USA) and statistical software package $R$ ( $R$ foundation, Vienna, Austria). Correlations between the different methods for MTL assessment and between MTL and other biomarkes (CSF, APOE-e4 allele) were analyzed by the Pearson's correlation coefficient for continuous data, and the Spearman rank test for correlations including the MTAscore. Intraclass correlation coefficient (ICC) and paired t-test were performed to investigate the agreement between MTL measurements.

The main outcome measure was the area rereareunder the curve (AUC) for AD-

56 | Chapter 3: MTL measurements for AD prediction in $\mathrm{MCl}$ 
type dementia after 2 years follow-up, calculated using a time-dependent ROC curve in R [59]. Differences in AUC between methods were tested as described elsewhere [60]. We calculated the sensitivity, specificity, positive predictive value (PPV), negative predictive value (NPV), odds ratio (OR) and hazards ratio (HR) for AD-type dementia at 2-year follow-up using data-driven cut-points based on a time dependent ROC. First we calculated the cut-point that maximized the Youden Index (sensitivity+specificity-1) for predicting AD-type dementia after 2 years. Second, we selected cut-points that predicted AD-type dementia with a sensitivity of $85 \%$. Cutpoints were calculated in the whole sample and in each cohort separately. Spline analyses were performed to determine AD-free survival after 2 year as a function of each MRI-measurement in the total cohort. Slope analyses with mixed models were performed to investigate whether MTL atrophy was associated with change on the MMSE and a cognitive composite score at follow-up. The analyses included the baseline score and available follow-up scores up to 5 years after baseline and were corrected for age and education. An unstructured covariance structure with center as a random effect was used as this model provided the best -2 log likelihood compared to models with simpler covariance structures [40].

\section{RESULTS}

\section{Subject characteristics}

From the total group of $\mathrm{MCl}$-patients included in this study $37 \%$ were non-amnestic $\mathrm{MCl}$ (73\% single-domain and $27 \%$ multi-domain) and $63 \%$ amnestic $\mathrm{MCl}(44 \%$ single-domain and $56 \%$ multi-domain). Baseline and follow-up characteristics are shown in table 1. Both cohorts were comparable for age and APOE-e4 status. The DESCRIPA cohort included more females and education was lower compared to the VUmc cohort. Scores on the MMSE and a delayed recall task were lower in the VUmc cohort. At follow-up, ninety-one subjects were diagnosed with probable Alzheimer type dementia (28\%). Conversion rate was higher in the VUmc cohort (35.5\%) than in the DESCRIPA cohort (19.2\%), $p<.001$. Twelve subjects converted to a different type of dementia at follow-up: 4 subjects converted to frontotemporal dementia, 6 to dementia with Lewy bodies, one to vascular dementia and one to another form of dementia. These subjects were included in the no-AD group. The follow-up length was slightly higher in the VUmc cohort. Characteristics of subjects with and without AD-type dementia at FU in each cohort are shown in supplementary table S2. 
Table 1. Subject characteristics.

\begin{tabular}{|c|c|c|c|}
\hline & Descripa cohort & VUmc cohort & Combined cohort \\
\hline N & 156 & 172 & 328 \\
\hline Age & $70.3(7.9)$ & $70.9(7.3)$ & $70.6(7.6)$ \\
\hline Female (\%) & 59.6 & $44.2 * *$ & 51.5 \\
\hline Years education & $8.5(3.9)$ & $11.2(3.3)^{* * *}$ & $10(3.8)$ \\
\hline MMSE score & $27.2(2.3)$ & $26.6(2.6)^{*}$ & $27.0(2.5)$ \\
\hline $\begin{array}{l}\text { Z-score wordlist (delayed } \\
\text { recall) }\end{array}$ & $-1.23(1.20)$ & $-1.58(1.10)^{*}$ & $-1.38(1.20)$ \\
\hline ApoE-e4-carrier (\%) & 45 & 56 & 51 \\
\hline $\begin{array}{l}\text { Homo/heterozygous } \\
\text { ApoE-e4-carriers (\%) }\end{array}$ & $38 / 7$ & $38 / 18$ & $38 / 13$ \\
\hline $\begin{array}{l}\text { Manual hippocampus } \\
\left(\mathrm{mm}^{3}\right)\end{array}$ & 7874 (1294) & $7462(1097)^{* *}$ & $7657(1211)$ \\
\hline $\begin{array}{l}\text { LEAP hippocampus } \\
\left(\mathrm{mm}^{3}\right)\end{array}$ & 5897 (798) & $5446(632)^{* * *}$ & 5661 (749) \\
\hline MTA-score & $2.8(1.7)$ & $2.3(1.7)^{* *}$ & $2.5(1.7)$ \\
\hline Lateral ventricle $\left(\mathrm{mm}^{3}\right)$ & 53409 (26105) & $57374(28042)$ & $55488(27170)$ \\
\hline CSF A $\beta 1-42, \mathrm{pg} / \mathrm{ml}$ & $561(256)$ & $604(283)$ & $594(277)$ \\
\hline $\begin{array}{l}\text { Abnormal CSF A } \beta 1-42 \\
(\%)\end{array}$ & 49 & 52 & 51 \\
\hline CSF t-tau, pg/ml & $418(298)$ & $558(347)^{*}$ & $525(351)$ \\
\hline Abnormal CSF t-tau (\%) & 51 & 68 & 64 \\
\hline CSF p-tau, pg/ml & $64(33)$ & $80(47)$ & $77(45)$ \\
\hline Abnormal CSF p-tau (\%) & 54 & 71 & 67 \\
\hline CSF Ratio A $\beta 1-42 /$ t-tau & $2.2(2.2)$ & $1.6(1.5)$ & $1.8(1.6)$ \\
\hline \multicolumn{4}{|l|}{ Outcome at last FU (\%) } \\
\hline - No AD & 80.8 & 64.5 & 72 \\
\hline$-A D$ & 19.2 & $35.5^{* * *}$ & 28 \\
\hline $\begin{array}{l}\text { Average FU non-de- } \\
\text { mented subjects (year) }\end{array}$ & $2.35(0.84)$ & $2.67(1.31)^{*}$ & 2.47 (1.09) \\
\hline $\begin{array}{l}\text { Average time to AD } \\
\text { (year) }\end{array}$ & $1.51(0.71)$ & $2.29(1.39)^{* *}$ & $2.07(1.28)$ \\
\hline
\end{tabular}

All volumetric measurements are corrected for intracranial volume. Values are mean (SD). Abbreviations: $\mathrm{MMSE}=$ Mini-Mental state examination; $\mathrm{ApoE}=$ apolipoprotein E genotype; 
$\mathrm{A} \beta 1-42=$ beta amyloid 1-42; $\mathrm{t}$-tau $=$ total tau, $\mathrm{p}$-tau $=$ phosphorylated tau; $\mathrm{FU}=$ followup; $\mathrm{AD}=$ Alzheimer's disease. ${ }^{*} p<.05, * * p<.01, * * * p<.001$ for differences between cohorts.

\section{Correlations between MTL-measures}

Manual and automated LEAP hippocampal volumes correlated highly (Pearson's r: $0.71, p<.001$ ) with intermediate agreement (ICC: 0.38, $p<.001$, Bland-Altmanplot (Supplementary Figure S2)). Paired t-test showed significant differences between the two techniques ( $\mathrm{t}: 42.27, p<.01$ ) with LEAP hippocampal measurement showing lower volumes. Using the cut-point based on the Youden index, the kappa and the overlap between manual and LEAP hippocampal volume were 0.60 and 0.80 respectively.

The correlation between the MTA-score and the manual and LEAP hippocampal volume was -0.36 and -0.27 respectively $(p<.01)$. Using the cut-point based on the Youden index, the kappa was 0.29 and the overlap between scores 0.69 for manual vs MTA-score and 0.37 (kappa) and 0.65 (overlap) for LEAP vs MTA-score. The lateral ventricle measurement strongly correlated with MTA-score $(r=0.60, p<.001)$ and showed weak correlations with both manual $(r=-0.20, p<.01)$ and LEAP hippocampal volume $(r=-0.20, p<.01)$.

\section{Correlations with AD biomarkers}

Manual and LEAP hippocampal volume significantly correlated with CSF t-tau, p-tau and $A \beta /$ t-tau ratio (all $p<.01$, Table 2 ). The MTA-score correlated with A $\beta 1-42(p<.05)$ and the ratio $A \beta /$ t-tau $(p<.01)$. The lateral ventricle volume correlated with $A \beta 1$ $42(p<.01)$ and $p$-tau $(p<.05)$. Only the LEAP hippocampal volume at baseline was associated with APOE-e4 allele status (LEAP volume APOE-e4 carriers $5516 \mathrm{~mm}^{3}$ vs $5852 \mathrm{~mm}^{3}$ for APOE-e4 non-carriers, $\mathrm{t}=2.72, p<.001$ ). 
Table 2. Correlation with CSF measurements.

\begin{tabular}{lcccc}
\hline & $\mathrm{A} \beta 1-42$ & $\mathrm{~T}$-tau & $\mathrm{P}$-tau & $\mathrm{A} \beta / \mathrm{t}$-tau \\
\hline Manual hippocampus & 0.09 & $-0.28^{* *}$ & $-0.23^{* *}$ & $0.29^{* *}$ \\
LEAP hippocampus & 0.05 & $-0.32^{* *}$ & $-0.27^{* *}$ & $0.33^{* *}$ \\
MTA-score & $-0.20^{*}$ & 0.11 & 0.06 & $-0.24^{* *}$ \\
Lateral ventricle & $-0.30^{* *}$ & 0.12 & $0.16^{*}$ & -0.1 \\
\hline
\end{tabular}

Shown are Pearson correlation coefficients or Spearman-rank correlation coefficients (correlations with MTA score). Abbreviations: $A \beta 1-42$ = beta amyloid 1-42; $\mathrm{t}$-tau = total tau; $\mathrm{p}$-tau $=$ phosphorylated tau; $\mathrm{MTA}=$ medial temporal lobe atrophy. ${ }^{*} \mathrm{p}<.05,{ }^{* *} \mathrm{p}<.01$.

\section{Predictors of AD-type dementia in combined sample}

In the total sample, the AUC for prediction of AD-type dementia at 2 years follow-up was highest for the LEAP hippocampal measurement (0.71) and manual hippocampal measurement (0.71), while it was substantially lower for the MTA-score (0.65) and lateral ventricle (0.60) (Figure 1). If measurements were dichotomised using the cutpoint that maximised the Youden Index, the overall predictive accuracy for AD after 2 years was best for the manual and LEAP hippocampal volumetric measurements (OR 6.4-6.5, HR 4.4-4.5), and lowest for the qualitative rating and lateral ventricle volume $(\mathrm{OR}<4, \mathrm{HR}<3$ ) (Table 3 ). Figure 2 shows the 2 -year dementia risk according to baseline MTL score based on spline analysis. The $x$-axis shows the degree of atrophy and the $y$-axis depicts the risk for $A D$ at follow-up associated with the degree of atrophy. Both manual and LEAP hippocampal volume had a non-linear relation with dementia risk indicating that the risk for dementia did not change much with very large or very small volumes. The optimal cut-point based on the Youden index for these measures was found halfway through the linear part of the plot.

If measurements were dichotomised using the cut-point that provided a sensitivity of $85 \%$ similar results were obtained. These analyses also showed that the specificity was higher for manual and LEAP volumetric measurements of hippocampal volume than for MTA-score and lateral ventricle volume. 


\section{Predictors of cognitive decline}

Since subjects with $\mathrm{MCl}$ may convert to AD-type dementia after the 2-years follow-up, we performed slope analyses with annual cognitive decline up to 5 years as outcome measure. Subjects with abnormal scores for manual and LEAP hippocampal measurement and MTA-score at baseline declined 2 times as fast on the MMSE $(p<.001)$ and declined 2.6 to 4 times as fast on the cognitive composite score $(p<.01)$ compared to subjects with normal MTL scores (Table 4, Figure 3$)$. The lateral ventricle volume only predicted decline on the cognitive composite score $(p<.01)$ (Table 4, Figure 3).

\section{Multicenter versus single-center cohort}

In the DESCRIPA multicenter cohort, AUC analysis showed best predictive accuracy values for LEAP (0.74) followed by manual hippocampus volume $(0.71)$. In the single-center cohort, manual hippocampus was the best predictor for AD (AUC: 0.69) followed by LEAP (AUC: 0.68 ) and MTA-score (AUC: 0.65) (Figure 1). In order to investigate whether optimal cut-points for predicting AD-type dementia differed between the multicenter and single center cohort, we calculated within each cohort the cut-point that maximised the Youden index and the cut-point that provided a sensitivity of $85 \%$ for the prediction of AD-type dementia after 2 year (Table S3). These analyses showed that the optimal cut-points for the manual and LEAP hippocampal measurement were similar between the multicenter and the single-center cohort with relative differences of less than $2.5 \%$ for the cut-points based on the Youden index and less than 5.5\% for the cut-points that provided a sensitivity of $85 \%$. 

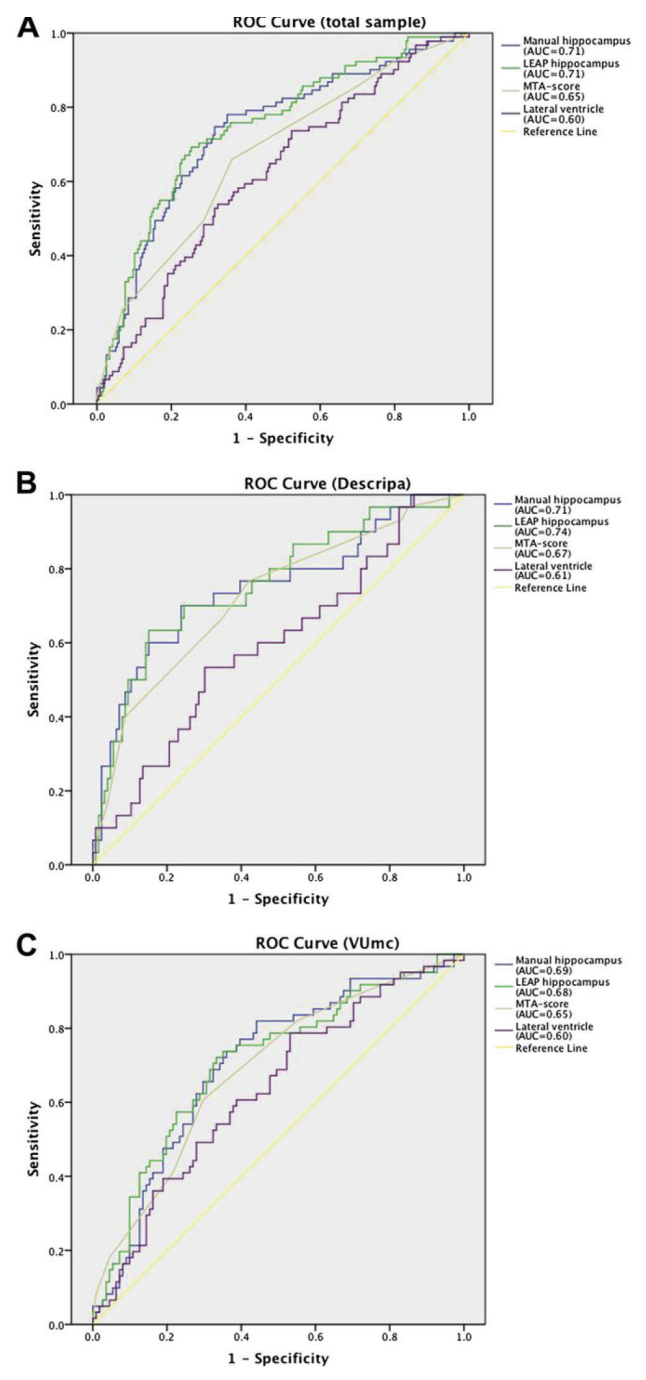

Fig. 1. Area under the curve (AUC) of a ROC-curve for MRI-measurements for $A$ ) total sample, B) DESCRIPA cohort and C) VUmc cohort. All volumetric measurements are corrected for intracranial volume. Abbreviations: $\mathrm{ROC}=$ receiver operating characteristics; MTA = medial temporal lobe atrophy. 
A

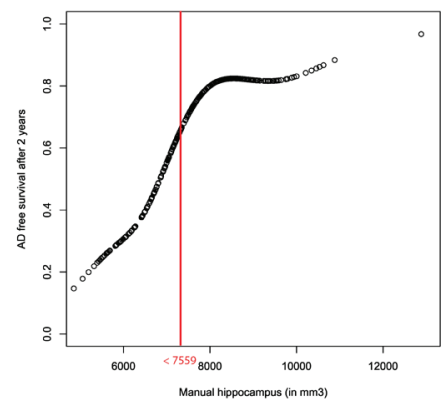

C

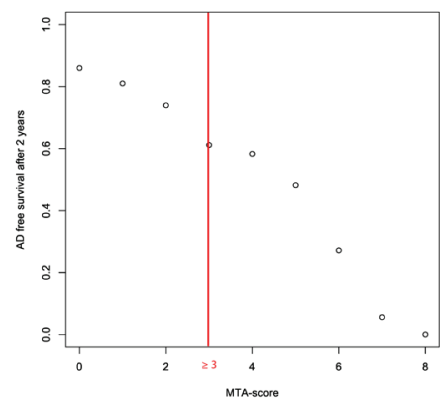

B

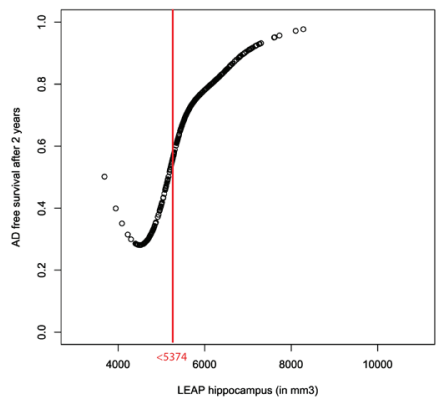

D

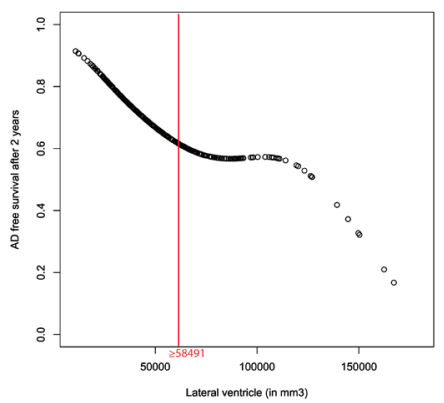

Fig. 2. Spline analyses showing AD-free survival after 2 year as function of MRImeasurement in total cohort for A) Manual hippocampal volume, B) LEAP hippocampal volume, C) MTA-score and D) Lateral ventricle volume. Red line indicates cut-point that maximised the Youden index. All volumetric measurements are corrected for intracranial volume. Abbreviations: AD = Alzheimer's disease; MTA $=$ medial temporal lobe atrophy. 
A
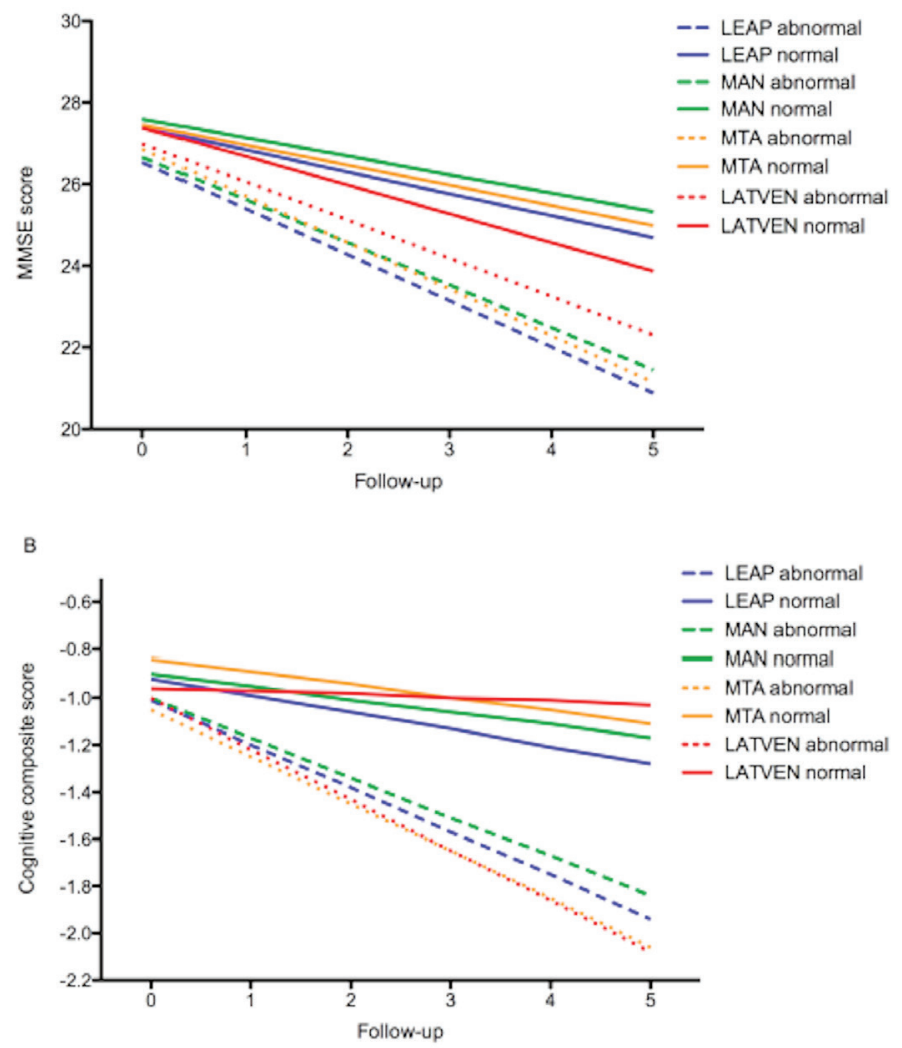

Fig. 3. Slope analyses showing annual cognitive decline on A) the MMSE and B) a cognitive composite score as predicted by baseline MTL measurements. Abnormal MRI-values at baseline were defined as manual hippocampal volume $<7559 \mathrm{~mm}^{3}$, LEAP hippocampal volume $<5374 \mathrm{~mm}^{3}$, MTA-score $\geq 3$ and lateral ventricle volume $\geq 58491 \mathrm{~mm}^{3}$. Figure $A+B$ : the solid line indicates the subjects with normal values. The dotted line indicates subjects with abnormal values. Abbreviations: $M A N=$ manual hippocampal volume; LEAP = LEAP hippocampal volume; MTA $=$ medial temporal lobe atrophy score; LATVEN = lateral ventricle volume; MMSE = Mini-Mental State Examination. All volumetric measurements are corrected for intracranial volume. The cognitive composite score $=$ average $z$-score of learning or delayed recall of a word list learning test or equivalent memory test, the trail making test (TMT) part $A$, TMT part B, verbal fluency, Rey figure copy test or an equivalent test.

64 | Chapter 3: MTL measurements for AD prediction in $\mathrm{MCl}$ 
Table 3. Predictive accuracy for AD-type dementia after 2-years.

\begin{tabular}{lccccccc}
\hline $\begin{array}{l}\text { Cut-point based on } \\
\text { Youden index }\end{array}$ & Cut-off & Sens & Spec & PPV & NPV & OR & HR \\
\hline Manual hippocampus & 7559 & 0.78 & 0.65 & 0.46 & 0.88 & 6.47 & 4.55 \\
LEAP hippocampus & 5374 & 0.66 & 0.77 & 0.52 & 0.85 & 6.4 & 4.43 \\
MTA-score & 3 & 0.66 & 0.64 & 0.41 & 0.83 & 3.4 & 2.77 \\
Lateral ventricle & 58491 & 0.53 & 0.68 & 0.39 & 0.79 & 2.36 & 2.01 \\
\hline Cut-point for a & & & & & & & \\
sensitivity of 85\% & & & & & & & \\
\hline Manual hippocampus & 8379 & 0.88 & 0.37 & 0.35 & 0.89 & 4.22 & 3.15 \\
LEAP hippocampus & 5901 & 0.87 & 0.42 & 0.36 & 0.89 & 4.72 & 3.34 \\
MTA-score & 2 & 0.86 & 0.3 & 0.32 & 0.85 & 2.57 & 2.03 \\
Lateral ventricle & 34859 & 0.84 & 0.27 & 0.31 & 0.81 & 1.87 & 2 \\
\hline
\end{tabular}

All volumetric measurements are corrected for intracranial volume. Cut-off in $\mathrm{mm}^{3}$ for volumetric measurements. Abbreviations: Sens = sensitivity; $\mathrm{Spec}=$ specificity; PPV= positive predictive value; NPV = negative predictive value; $A U C$ = area under the curve; $O R$ = odds ratio; $\mathrm{HR}$ = hazard ratio; LEAP = Learning Embeddings for Atlas Propagation; MTA = Medial temporal lobe atrophy.

\section{DISCUSSION}

The present study showed that volumetric measurements of the MTL are better predictors for AD-type dementia in subjects with $\mathrm{MCl}$ than a qualitative rating or the assessment of the lateral ventricle volume.

This is to our knowledge the first study comparing four different measures for MTL atrophy in a large sample of $\mathrm{MCl}$-subjects from a memory clinic population. Furthermore this is the first study to compare the predictive accuracy of these measurements in single-center and multicenter settings and to investigate the correlations between these measurements and other AD biomarkers.

\section{Comparison of MTL assessment methods}

Predictive accuracy

The degree of neurodegeneration in MTL structures is the best MRI marker of imminent conversion to $A D$, with decreased hippocampal volume being the most robust structural MRI feature [61]. 
Table 4. Annual cognitive decline over 5 years of follow-up.

\begin{tabular}{|c|c|c|c|c|c|}
\hline & & \multicolumn{2}{|l|}{ MMSE score } & \multicolumn{2}{|c|}{ Cognitive composite score } \\
\hline & & $\begin{array}{l}\text { Baseline } \\
\text { score }\end{array}$ & Slope & $\begin{array}{l}\text { Baseline } \\
\text { score }\end{array}$ & Slope \\
\hline \multirow{2}{*}{$\begin{array}{l}\text { Manual } \\
\text { hippocampus }\end{array}$} & Normal & $27.6(0.47)^{\mathrm{a}}$ & $-0.45(0.09)^{a, b}$ & $-0.90(0.08)$ & $-0.05(0.02)^{c, d}$ \\
\hline & Abnormal & $26.7(0.47)$ & $-1.04(0.10)^{\mathrm{e}}$ & $-1.00(0.09)$ & $-0.17(0.03)^{\mathrm{e}}$ \\
\hline \multirow{2}{*}{$\begin{array}{l}\text { LEAP } \\
\text { hippocampus }\end{array}$} & Normal & $27.4(0.42)^{c}$ & $-0.54(0.09)^{a, b}$ & $-0.92(0.07)$ & $-0.07(0.02)^{b, c}$ \\
\hline & Abnormal & $26.5(0.46)$ & $-1.13(0.12)^{\mathrm{e}}$ & $-1.01(0.09)$ & $-0.18(0.04)^{e}$ \\
\hline \multirow[t]{2}{*}{ MTA-score } & Normal & $27.4(0.43)^{f}$ & $-0.49(0.08)^{a, e}$ & $-0.84(0.07)^{c}$ & $-0.05(0.02)^{a, b}$ \\
\hline & Abnormal & $26.9(0.43)$ & $-1.14(0.11)^{\mathrm{e}}$ & $-1.05(0.08)$ & $-0.20(0.03)^{e}$ \\
\hline \multirow{2}{*}{$\begin{array}{l}\text { Lateral } \\
\text { ventricle }\end{array}$} & Normal & $27.4(0.46)$ & $-0.70(0.09)^{\mathrm{e}}$ & $-0.96(0.07)$ & $-0.01(0.03)^{c}$ \\
\hline & Abnormal & $27.0(0.48)$ & $-0.94(0.13)^{\mathrm{e}}$ & $-1.00(0.09)$ & $-0.22(0.05)^{\mathrm{c}, \mathrm{e}}$ \\
\hline
\end{tabular}

Data are mean (SD). Slope refers to annual change on the test. A negative slope indicates cognitive decline. Scores dichotomised based on Youden cut-offs.

Key: MMSE=Mini-Mental State Examination; MTA = medial temporal lobe atrophy ${ }^{a} p<0.001$ for baseline score or slope compared with the abnormal biomarker group.

${ }^{\mathrm{b}} p<0.01$ for slope different from 0 (=a statistically significant change over time in test score). ${ }^{c} p<0.01$ for baseline score or slope compared with the abnormal biomarker group.

${ }^{\mathrm{d}} p<0.05$ for slope different from 0 (=a statistically significant change over time in test score). ${ }^{\mathrm{e}} p<0.001$ for slope different from 0 (=a statistically significant change over time in test score). ${ }^{f} p<0.05$ for baseline score or slope compared with the abnormal biomarker group.

In our study, predictive accuracy of both volumetric hippocampal measurements for AD-type dementia was indeed higher than that of the qualitative rating and lateral ventricle measure. This is in line with current evidence stating that manual 
and automated volumetric methods show similar performances in diagnosing $A D$ $[25,31,62,63]$ and that qualitative rating scales or lateral ventricle measurements are less accurate predictors than volumetric methods $[24,28,30]$.

The slope analyses of cognitive decline over 5 years again yielded similar results with LEAP and manual hippocampal volume at baseline predicting cognitive decline on both the MMSE and a cognitive composite score. Slope analysis for the MTAscore predicted cognitive decline equally well as the volumetric measures. This is again in line with previous findings showing that automatically measured volume change in the hippocampus is correlated with decline of performance on the MMSE [64] and that manually measured hippocampal volume reduction is correlated with the severity of impairment on neuropsychological tests [65]. Another study found that performance on the MMSE was directly correlated with hippocampal volume [66].

\section{Relation with other AD-biomarkers}

Manual and LEAP hippocampal volume significantly correlated with CSF t-tau and $p$-tau but not with A $A 1-42$. The correlation of hippocampal volumetric measurements with CSF tau but not with CSF $A \beta$ is in line with previous studies conducted in subjects with $\mathrm{MCl}[67,68]$ and subjects with prodromal $A D$ or $A D$ [69]. It may be explained by the obervation that antemortem hippocampal volume significantly correlated with the density of neurofibrillary tangles at autopsy [70, 71] but not with amyloid beta plaque load [70]. The qualitative MTA-score and lateral ventricle volume correlated with CSF A $\beta 1-42$. This correlation may indicate that these MTL measures in part reflect generalised brain atrophy as previous studies showed that lower $A \beta 1-42$ levels but not $t$-tau levels were associated with total brain atrophy and ventricular volume [72].

\section{Overlap between measurements}

Although the LEAP and manual hippocampal measurement correlated highly $(r=0.71)$ and scores showed $80 \%$ overlap, the intraclass correlation coefficient (ICC) of 0.38 indicated a moderate agreement. This might be due to the fact that the LEAP volume was consistently lower, since the ICC is sensitive for absolute sizes. A previous study that compared LEAP with manual measurements found a much higher ICC of 0.89 [17]. Although in this study LEAP volume was systematically lower 
than the manual volumetric measurement, this difference was smaller than in our study. Differences in absolute volumes may be due to the use of different borders for hippocampal delineation. Major parts of the hippocampus are included in both volumetric methods with the only difference that the LEAP method misses some of the hippocampal head, some of the alveus and some of the fimbria, and ends a few slices earlier than manual hippocampal outlining. Despite the differences in raw volume, both methods have a comparable diagnostic accuracy indicating that these parts are less important for AD pathology. It is also likely that the cut-off points for each hippocampal method likely reflected a similar degree of abnormality. Namely, if we defined the cut-off that provided a sensitivity of 0.85 , the specificity of each method was equal. When the cut-off was used that optimised the Youden index, small differences in sensitivity and specificity were found but the combination of sensitivity and specificity expressed as odds ratio was the same. Differences in absolute volumens between different measurements protocols may be reduced in the future as efforts are made towards the harmonization of an MRI segmentation protocol for hippocampal delineation [20]. Since manual volumetry is used as the standard against which automated segmentation algorithms are assessed, future synchronization and comparison of both techniques will be facilitated. Correlations between manual and LEAP volumetric measurements and MTA-score were low with a moderate overlap between both methods. The same pattern was found for correlations and overlap between both volumetric hippocampal measurements and expansion of the lateral ventricle.

The findings above indicate, in line with previous studies, that volumetric and qualitative measures of MTL atrophy measure different aspects of AD pathology $[73,74]$. Manual and LEAP hippocampal volume correlated with each other, both showed high predictive accuracy values and both correlated with $\mathrm{t}$-tau and $\mathrm{p}$-tau but not with $A \beta 1-42$. In contrast, visually rated MTA-score and expansion of the lateral ventricle correlated highly with each other but not with the volumetric measures. They showed lower predictive accuracy for AD-conversion than the volumetric measures and correlated highly with amyloid beta in CSF rather than CSF tau. As discussed above, it is possible that both the MTA-score and lateral ventricle volume reflect a widening of ventricular spaces i.e. the temporal horn, which may be indicative of generalised atrophy rather than atrophy of the hippocampus alone. 


\section{Comparison of cohorts}

None of the MTL measures showed major differences in predictive accuracy between the single and multicenter cohort. When optimal cut-points for each measure were calculated for each cohort separately these cut-points only slightly differed between the cohorts for the volumetric hippocampal measurements, which shows that volumetric measurements display stable cut-points across different cohorts Some differences were noted between the cohorts on cut-points for the MTA-score and lateral ventricle, suggesting that MTA-score and lateral ventricle are more sensitive for cohort differences.

\section{Technical considerations}

In general automated measurements are more susceptible to scanner and scan protocol variability. In our study, no significant differences between both methods were found with LEAP showing similar or even slightly better performance than manual volumetry. This suggests that automated measurements can be performed in multi-centre studies without strict standardisation of scan protocols. The percentage of technical failures was small for manual hippocampal measurement $(0.07 \%)$ and LEAP hippocampal measurement $(0.03 \%)$. All failures were observed in 2 DESCRIPA sites that apparently used scan frequencies which occasionally led to technical errors during the pre-processing phase (e.g. intensity problems), making these particular scans unsuitable for applying subsequent volumetric measurements. SIENAX related measurements (ICV measure and lateral ventricular volume) yielded a higher technical failure rate ( $1 \%$ for lateral ventricle) and were observed across all sites. These were due to calculation errors or a combination of software and image quality problems. These findings indicate that the lateral ventricle measurement is more sensitive for specific differences in image quality than a manual or automated hippocampal measurement. It should be noted than scan sequences were designed for routine clinical practice and not for automated measurements and it is likely that failure rate could be reduced by using protocols optimised for automated measurements.

\section{Limitations}

The present study had several limitations. There were differences in subject characteristics between the single-center and multicenter cohort. Subjects from 
the single-center VUmc cohort were more severely impaired and had lower baseline MMSE scores, more severe memory impairment, a lower hippocampal volume, higher CSF tau, and a higher conversion rate to dementia. Despite these differences, however, the predictive accuracy was similar in each cohort. Another limitation is that the cut-offs of all MRI measurements were determined within a study population that also included the subjects from the present analyses. This could have led to an overestimation of the predictive accuracy. However, it is unlikely that it influenced our findings with respect to the differences in predictive accuracy between MRI measurements, as we used the same method to define the cut-point for each measurement. Follow-up data with $A D$ diagnosis for all subjects was only available for relatively short follow-up intervals (2 years). For clinical trials, however, short-term prognosis may be important. For those subjects of whom longterm follow-up data was available, predictive accuracy for annual cognitive decline up to 5 years was additionally investigated. The diagnosis of $A D$ at follow-up was not validated neuropathologically which may have possibly led to the misclassification of some cases. We used scanners with different field strengths (1T and 1.5T) which reflected real-life situations in which scanners and magnetic field strengths do vary. However, these differences may have introduced bias. We therefore compared the hippocampal volume between subjects scanned on a 1.0T scanner $(n=127)$ and a 1.5T scanner $(n=201)$ after correction for age, gender, educational level, baseline MMSE score and follow-up diagnosis. The difference in volume between 1.0 and 1.5 T scanners was $0.2 \%$ for the LEAP method ( $p=0.9,[75]), 0.9 \%$ for manual hippocampal volume $(p=0.6)$, and $1.5 \%$ for the lateral ventricle volume $(p=0.8)$. We also tested whether field strength modulated the effect of the volumetric measures on conversion to AD-type dementia. For none of the measures the interaction between field strength and volumetric measure was statistically significantly associated with conversion $(p>0.15)$. A recent study also found a limited effect of fieldstrength on hippocampal volume. This study compared the hippocampal volume measured by the LEAP method between subjects scanned both on a 1.5T and 3T scanner (Wolz 2013, under review). This study found a very high correlation between the measurements on each scanner $(r=0.98)$. The volumes measured on 3T were on average $24.4 \mathrm{~mm}^{3}$ or $1.17 \%$ larger than on $1.5 \mathrm{~T}$ (Wolz 2013, under review). This variability was similar to that for volumes rescanned on scanners with the same field strength (1.5\%) (Wolz 2013, under review). Taken together, it is unlikely that the small difference in field strength in our study had a major effect 
on the volumetric measurements in our study. Different methods were used for ICV correction of both hippocampal measurements (see 2.3.2). Both correction methods were thoroughly compared and showed high correlations (ICC: 0.93 for LEAP with MNI versus FSL scaling and 0.95 for manual volumetric measurement with MNI versus FSL scaling) and similar results regarding predictive accuracy. As a result of this analysis, ICV correction of each individual method was applied. It can be considered a strength that for this diagnostic study a population from a memory clinic setting was used. A consequence however is that these findings may not be applicable to other settings, including the general population.

\section{Clinical implications}

Volumetric measurements of the MTL are the best predictors for AD-type dementia in subjects with $\mathrm{MCl}$. Both volumetric measurements strongly correlate with CSF markers of neuronal injury (CSF t-tau and $\mathrm{p}$-tau), are able to predict cognitive decline and show consistent cut-off values between different cohorts. LEAP hippocampal volume has the advantage over manual volumetry that it needs much less rater time and shows no interrater variability effects. In addition, LEAP has a low technical failure rate. Visual rating scales are also quick and easy to perform but show lower predictive accuracy rates and higher inter and intraindividual variability effects [23] compared to LEAP volumetric measurement. Another disadvantage of visual rating scales, although outside the scope of this study, is that they cannot detect subtle atrophy progression and are thus insensitive to change over time [24, 28]. Since the hippocampus is among the first areas affected by the disease [76, 77], repeated measurement of its volume is clinically important. LEAP hippocampal measurement is suitable for implementation in clinical practice with on average four minutes control time on a standard computer. Cut-points that maximised the balance between sensitivity and specificity as expressed by the Youden index or that provided a sensitivity of $85 \%$ were defined. The cut-point based on the Youden index may be preferred since it has shown to be more consistent between the multi and single centre cohort (Supplementary Table S3). Depending on the clinical needs also other cut-points may be chosen, that for example maximize the positive or negative predictive value [78]. Any choice for a specific cut-point has a trade-off between the chance of missing the disease (false negative rate, 1-sensitivity) or incorrectly diagnosing someone as having the disease (false positive rate, 1-specificity). The choice will therefore depend on its clinical use. For example, if a treatment for $\mathrm{MCl}$ 
due to $A D$ would be available the choice may depend on the safety profile of the treatment. If a treatment has many or severe side-effects, high specificity is more important than a high sensitivity and the opposite is true for treatments with little side-effects. In the situation that treatment is not available and biomarkers are used for diagnosis, one may prefer to use a cut-point with a low false positive rate as an incorrect diagnosis of $A D$ may have a major negative impact on the patient.

\section{Future directions}

Future MRI-studies need to investigate abnormalities in AD signature regions in and outside the MTL. A recent study found that subjects with future cognitive impairment (preclinical $A D$ and $\mathrm{MCl}$ ) also showed reduced brain volume in posterior cingulate/ precuneus and orbitofrontal cortex, at least 4 years before any cognitive symptoms [79]. Other structural MR studies also found abnormalities in $A D$ or $\mathrm{MCl}$ outside the MTL region such as the corpus callosum [80-83], cingulum [84-86], parietal [87], temporal lobe other than MTL [88] and frontal lobe [89]. Future MRI-studies need to investigate abnormalities in AD signature regions in and outside the MTL.

\section{CONCLUSION}

Volumetric hippocampal measurements are the best predictors of conversion to ADtype dementia in subjects with $\mathrm{MCl}$ after 2 years follow-up and are able to predict annual cognitive decline. For the limited rater time, LEAP automated hippocampal measurement may be preferred. 


\section{REFERENCES}

1. Masters CL, Beyreuther K. Alzheimer's centennial legacy: prospects for rational therapeutic intervention targeting the Abeta amyloid pathway. Brain 2006;129:2823-2839.

2. Vellas B, Andrieu S, Sampaio C, Wilcock G. Disease-modifying trials in Alzheimer's disease: a European task force consensus. The Lancet Neurology 2007;6:56-62.

3. Visser PJ, Verhey FRJ, Hofman PAM, Jolles J. Medial temporal lobe atrophy predicts Alzheimer's disease in patients with minor cognitive impairment. J Neurol Neurosurg Psychiatry 2002;72:491-497.

4. Rusinek HP, Endo YM, De Santi SP, et al. Atrophy rate in medial temporal lobe during progression of Alzheimer disease. Neurology 2004;63:2354-2359.

5. Schoonenboom NSM, van der Flier WM, Blankenstein MA, et al. CSF and MRI markers independently contribute to the diagnosis of Alzheimer's disease. Neurobiology of Aging 2008;29:669-675.

6. Visser PJ, Scheltens P, Verhey FRJ, et al. Medial temporal lobe atrophy and memory dysfunction as predictors for dementia in subjects with mild cognitive impairment. Journal of Neurology 1999;246:477-485.

7. DeCarli C, Frisoni GB, Clark CM, et al. Qualitative Estimates of Medial Temporal Atrophy as a Predictor of Progression From Mild Cognitive Impairment to Dementia. Arch neurology 2007;64:108-115.

8. Aljabar P, Heckemann RA, Hammers A, Hajnal JV, Rueckert D. Multi-atlas based segmentation of brain images: Atlas selection and its effect on accuracy. Neurolmage 2009;46:726-738.

9. Apostolova LG, Dinov ID, Dutton RA, et al. 3D comparison of hippocampal atrophy in amnestic mild cognitive impairment and Alzheimer's disease. Brain 2006;129:2867-2873.

10. Chou Y-Y, Lepore N, Saharan P, et al. Ventricular maps in 804 ADNI subjects: correlations with CSF biomarkers and clinical decline. Neurobiology of Aging 2010;31:1386-1400.

11. Echávarri C, Aalten $\mathrm{P}$, Uylings HBM, et al. Atrophy in the parahippocampal gyrus as an early biomarker of Alzheimer's disease. Brain Structure and Function 2011;215:265-271.

12. Jack CR, Shiung MM, Gunter JL, et al. Comparison of different MRI brain atrophy 
rate measures with clinical disease progression in AD. Neurology 2004;62:591600.

13. McHugh TL, Saykin AJ, Wishart HA, et al. Hippocampal Volume and Shape Analysis in an Older Adult Population. The Clinical Neuropsychologist 2007;21:130-145.

14. Nestor SM, Rupsingh R, Borrie M, et al. Ventricular enlargement as a possible measure of Alzheimer's disease progression validated using the Alzheimer's disease neuroimaging initiative database. Brain 2008;131:2443-2454.

15. Scheltens P, Leys D, Barkhof F, et al. Atrophy of medial temporal lobes on MRI in "probable" Alzheimer's disease and normal ageing: diagnostic value and neuropsychological correlates. J Neurol Neurosurg Psychiatry 1992;55:967972.

16. Varela-Nallar L, Aranguiz FC, Abbott AC, Slater PG, Inestrosa NC. Adult hippocampal neurogenesis in aging and Alzheimer's disease. Birth Defects Research Part C: Embryo Today: Reviews 2010;90:284-296.

17. Wolz R, Aljabar P, Hajnal JV, Hammers A, Rueckert D. LEAP: Learning embeddings for atlas propagation. Neurolmage 2010;49:1316-1325.

18. Wolz R, Heckemann RA, Aljabar P, et al. Measurement of hippocampal atrophy using $4 \mathrm{D}$ graph-cut segmentation: Application to ADNI. Neurolmage 2010;52:109-118.

19. Barnes J, Ourselin S, Fox NC. Clinical application of measurement of hippocampal atrophy in degenerative dementias. Hippocampus 2009;19:510-516.

20. Boccardi M, Ganzola R, Bocchetta M, et al. Survey of Protocols for the Manual Segmentation of the Hippocampus: Preparatory Steps Towards a Joint EADCADNI Harmonized Protocol. Journal of Alzheimer's Disease 2011;26:61-75.

21. van de Pol LA, Barnes J, Scahill RI, et al. Improved reliability of hippocampal atrophy rate measurement in mild cognitive impairment using fluid registration. Neurolmage 2007;34:1036-1041.

22. Galton CJ, Gomez-Anson B, Antoun N, et al. Temporal lobe rating scale: application to Alzheimer's disease and frontotemporal dementia. Journal of Neurology, Neurosurgery \& Psychiatry 2001;70:165-173.

23. DeCarli CM, Kaye JAM, Horwitz BP, Rapoport SIM. Critical analysis of the use of computer-assisted transverse axial tomography to study human brain in aging and dementia of the Alzheimer type. Neurology 1990;40:872-883.

24. Ridha BH, Barnes J, van de Pol LA, et al. Application of Automated Medial

74 | Chapter 3: MTL measurements for AD prediction in $\mathrm{MCl}$ 
Temporal Lobe Atrophy Scale to Alzheimer Disease. Archives of Neurology 2007;64:849-854.

25. Lehmann M, Douiri A, Kim LG, et al. Atrophy patterns in Alzheimer's disease and semantic dementia: A comparison of FreeSurfer and manual volumetric measurements. Neurolmage 2010;49:2264-2274.

26. Sanchez-Benavides G, Gomez-Ansun B, Sainz A, Vives Y, Delfino M, PeoaCasanova J. Manual validation of FreeSurfer's automated hippocampal segmentation in normal aging, mild cognitive impairment, and Alzheimer Disease subjects. Psychiatry Research: Neuroimaging 2010;181:219-225.

27. Shen L, Saykin A, Kim S, et al. Comparison of Manual and Automated Determination of Hippocampal Volumes in $\mathrm{MCl}$ and Early AD. Brain Imaging and Behavior 2010;4:86-95.

28. Urs R, Potter E, Barker W, et al. Visual Rating System for Assessing Magnetic Resonance Images: A Tool in the Diagnosis of Mild Cognitive Impairment and Alzheimer Disease. Journal of Computer Assisted Tomography 2009;33.

29. Wahlund L-O, Julin P, Johansson S-E, Scheltens P. Visual rating and volumetry of the medial temporal lobe on magnetic resonance imaging in dementia: a comparative study. Journal of Neurology, Neurosurgery \& Psychiatry 2000;69:630-635.

30. Westman E, Cavallin L, Muehlboeck JS, et al. Sensitivity and Specificity of Medial Temporal Lobe Visual Ratings and Multivariate Regional MRI Classification in Alzheimer's Disease. PLoS ONE 2011;6:e22506.

31. Hsu Y-Y, Schuff N, Du A-T, et al. Comparison of automated and manual MRI volumetry of hippocampus in normal aging and dementia. Journal of Magnetic Resonance Imaging 2002;16:305-310.

32. Carmichael OT, Aizenstein HA, Davis SW, et al. Atlas-based hippocampus segmentation in Alzheimer's disease and mild cognitive impairment. Neurolmage 2005;27:979-990.

33. Sanchez-Benavides G, Gomez-Anson B, Molinuevo JL, et al. Medial Temporal Lobe Correlates of Memory Screening Measures in Normal Aging, MCI, and AD. Journal of Geriatric Psychiatry and Neurology 2010;23:100-108.

34. Levy-Cooperman N, Ramirez J, Lobaugh NJ, Black SE. Misclassified Tissue Volumes in Alzheimer Disease Patients With White Matter Hyperintensities. Stroke 2008;39:1134-1141.

35. Visser PJ, Verhey FRJ, Boada M, et al. Development of Screening Guidelines and Chapter 3: MTL measurements for AD prediction in $\mathrm{MCl} \mid 75$ 
Clinical Criteria for Predementia Alzheimer,Äôs Disease. Neuroepidemiology 2008;30:254-265.

36. Morris JC. The Clinical Dementia Rating (CDR): current version and scoring rules. Neurology 1993;43:2412-2414.

37. Petersen RC. Mild cognitive impairment as a diagnostic entity. Journal of Internal Medicine 2004;256:183-194.

38. Petersen RC, Smith GE, Waring SC, Ivnik RJ, Tangalos EG, Kokmen E. Mild Cognitive Impairment: Clinical Characterization and Outcome. Archives of Neurology 1999;56:303-308.

39. Vos S, van Rossum I, Burns L, et al. Test sequence of CSF and MRI biomarkers for prediction of AD in subjects with $\mathrm{MCl}$. Neurobiology of Aging 2012.

40. Visser PJ, Verhey F, Knol DL, et al. Prevalence and prognostic value of CSF markers of Alzheimer's disease pathology in patients with subjective cognitive impairment or mild cognitive impairment in the DESCRIPA study: a prospective cohort study. The Lancet Neurology 2009;8:619-627.

41. APA APA. Diagnostic and Statistical Manual of Mental Disorders, 4th edition. Washington, D.C., 1994.

42. McKhann G, Drachman D, Folstein M, Katzman R, Price D, Stadlan EM. Clinical diagnosis of Alzheimer's disease: report of the NINCDS-ADRDA Work Group under the auspices of Department of Health and Human Services Task Force on Alzheimer's Disease. Neurology 1984;34:939-944.

43. Jack CR. MRI-Based Hippocampal Volume Measurements in Epilepsy. Epilepsia 1994;35:S21-S29.

44. van de Pol LA, van der Flier WM, Korf ESC, Fox NC, Barkhof F, Scheltens P. Baseline predictors of rates of hippocampal atrophy in mild cognitive impairment. Neurology 2007;69:1491-1497.

45. van de Pol LA, Verhey F, Frisoni GB, et al. White matter hyperintensities and medial temporal lobe atrophy in clinical subtypes of mild cognitive impairment: the DESCRIPA study. Journal of Neurology, Neurosurgery \& Psychiatry 2009;80:1069-1074.

46. Sluimer JD, van der Flier WM, Karas GB, et al. Whole-Brain Atrophy Rate and Cognitive Decline: Longitudinal MR Study of Memory Clinic Patients1. Radiology 2008;248:590-598.

47. Smith SM, De Stefano N, Jenkinson M, Matthews PM. Normalized Accurate Measurement of Longitudinal Brain Change. Journal of Computer Assisted 
Tomography 2001;25:466-475.

48. Smith SM, Zhang $Y$, Jenkinson $M$, et al. Accurate, Robust, and Automated Longitudinal and Cross-Sectional Brain Change Analysis. Neurolmage 2002;17:479-489.

49. Smith SM, Jenkinson M, Woolrich MW, et al. Advances in functional and structural MR image analysis and implementation as FSL. Neurolmage 2004;23:S208-S219.

50. Smith SM. Fast robust automated brain extraction. Human Brain Mapping 2002;17:143-155.

51. Jenkinson M, Smith S. A global optimisation method for robust affine registration of brain images. Medical Image Analysis 2001;5:143-156.

52. Jenkinson M, Bannister P, Brady M, Smith S. Improved Optimization for the Robust and Accurate Linear Registration and Motion Correction of Brain Images. Neurolmage 2002;17:825-841.

53. Zhang Y, Brady M, Smith S. Segmentation of brain MR images through a hidden Markov random field model and the expectation-maximization algorithm. Medical Imaging, IEEE Transactions on 2001;20:45-57.

54. Henneman WJP, Sluimer JD, Cordonnier C, et al. MRI Biomarkers of Vascular Damage and Atrophy Predicting Mortality in a Memory Clinic Population. Stroke 2009;40:492-498.

55. Andreasen N, Hesse C, Davidsson P, et al. Cerebrospinal Fluid \{beta\}Amyloid(1-42) in Alzheimer Disease: Differences Between Early- and LateOnset Alzheimer Disease and Stability During the Course of Disease. Archives of Neurology 1999;56:673-680.

56. Blennow K, Wallin A, Ågren H, Spenger C, Siegfried J, Vanmechelen E. Tau protein in cerebrospinal fluid: a biochemical diagnostic marker for axonal degeneration in Alzheimer's disease? . Mol Chem Neuropathol 1995;26:231245.

57. Kolen M BR. Test Equating: Methods and Practices. New York: Springer, 1995.

58. Mulder C, Verwey NA, van der Flier WM, et al. Amyloid- $\beta(1-42)$, Total Tau, and Phosphorylated Tau as Cerebrospinal Fluid Biomarkers for the Diagnosis of Alzheimer Disease. Clinical Chemistry 2010;56:248-253.

59. Heagerty PJ, Lumley T, Pepe MS. Time-Dependent ROC Curves for Censored Survival Data and a Diagnostic Marker. Biometrics 2000;56:337-344.

60. Hanley JA, McNeil BJ. A method of comparing the areas under receiver operating Chapter 3: MTL measurements for AD prediction in $\mathrm{MCl} \mid 77$ 
characteristic curves derived from the same cases. Radiology 1983;148:839843.

61. Risacher SL, Saykin AJ, West JD, et al. Baseline MRI Predictors of Conversion from $\mathrm{MCl}$ to Probable AD in the ADNI Cohort. Curr Alzheimer Res 2009;6:347361.

62. Colliot $\mathrm{O}$, Chetelat $\mathrm{G}$, Chupin $\mathrm{M}$, et al. Discrimination between Alzheimer Disease, Mild Cognitive Impairment, and Normal Aging by Using Automated Segmentation of the Hippocampus. Radiology 2008;248:194-201.

63. Sanchez-Benavides G, Gomez-Anson B, Sainz A, Vives Y, Delfino M, PenaCasanova J. Manual validation of FreeSurfer's automated hippocampal segmentation in normal aging, mild cognitive impairment, and Alzheimer Disease subjects. Psychiatry Res 2010;181:219-225.

64. Arlt S, Buchert R, Spies L, Eichenlaub M, Lehmbeck JT, Jahn H. Association between fully automated MRI-based volumetry of different brain regions and neuropsychological test performance in patients with amnestic mild cognitive impairment and Alzheimer's disease. EUROPEAN ARCHIVES OF PSYCHIATRY AND CLINICAL NEUROSCIENCE 2012.

65. Yavuz BB, Ariogul S, Cankurtaran M, et al. Hippocampal atrophy correlates with the severity of cognitive decline. International Psychogeriatrics 2007;19:767777.

66. Laakso MP, Soininen H, Partanen K, et al. Volumes of hippocampus, amygdala and frontal lobes in the MRI-based diagnosis of early Alzheimer's disease: Correlation with memory functions. Journal of Neural Transmission - Parkinson's Disease and Dementia Section 1995;9:73-86.

67. Apostolova LG, Hwang KS, Andrawis JP, et al. 3D PIB and CSF biomarker associations with hippocampal atrophy in ADNI subjects. Neurobiology of Aging 2010;31:1284-1303.

68. Carmichael O, Xie J, Fletcher E, Singh B, DeCarli C. Localized hippocampus measures are associated with Alzheimer pathology and cognition independent of total hippocampal volume. Neurobiology of Aging 2012;33:1124-1141.

69. de Souza LC, Chupin M, Lamari F, et al. CSF tau markers are correlated with hippocampal volume in Alzheimer's disease. Neurobiology of Aging 2011.

70. Csernansky JGMD, Hamstra JBS, Wang LP, et al. Correlations Between Antemortem Hippocampal Volume and Postmortem Neuropathology in AD Subjects. Alzheimer Disease \& Associated Disorders October/November/ 
December 2004;18:190-195.

71. Jack J, C.R., Dickson DW, Parisi JE, et al. Antemortem MRI Findings Correlate with Hippocampal Neuropathology in Typical Aging and Dementia. Neurology 2002;58:750-757.

72. Wahlund L-O, Blennow K. Cerebrospinal fluid biomarkers for disease stage and intensity in cognitively impaired patients. Neuroscience Letters 2003;339:99102.

73. Visser PJ, Verhey FRJ, Hofman PAM, Scheltens P, Jolles J. Medial temporal lobe atrophy predicts Alzheimer's disease in patients with minor cognitive impairment. Journal of Neurology, Neurosurgery \& Psychiatry 2002;72:491497.

74. Wahlund L-O, Julin P, Lindqvist J, Scheltens P. Visual assessment of medial temporal lobe atrophy in demented and healthy control subjects: correlation with volumetry. Psychiatry Research: Neuroimaging 1999;90:193-199.

75. van Rossum IA, Vos SJ, Burns $L$, et al. Injury markers predict time to dementia in subjects with $\mathrm{MCl}$ and amyloid pathology. Neurology 2012;79:1801-1816.

76. Chupin M, Gérardin E, Cuingnet R, et al. Fully automatic hippocampus segmentation and classification in Alzheimer's disease and mild cognitive impairment applied on data from ADNI. Hippocampus 2009;19:579-587.

77. Lötjönen J, Wolz R, Koikkalainen J, et al. Fast and robust extraction of hippocampus from MR images for diagnostics of Alzheimer's disease. Neurolmage 2011;56:185-196.

78. Bartlett JW, Frost C, Mattsson N, et al. Determining cut-points for Alzheimer,Äôs disease biomarkers: statistical issues, methods and challenges. Biomarkers in Medicine 2012;6:391-400.

79. Tondelli M, Wilcock GK, Nichelli P, De Jager CA, Jenkinson M, Zamboni G. Structural MRI changes detectable up to ten years before clinical Alzheimer's disease. Neurobiology of Aging 2012;33:825-836.

80. Di Paola M, Di lulio F, Cherubini A, et al. When, where, and how the corpus callosum changes in $\mathrm{MCl}$ and $\mathrm{AD}$ : A multimodal MRI study. Neurology 2010;74:1136-1142.

81. Chen T-F, Lin C-C, Chen Y-F, et al. Diffusion tensor changes in patients with amnesic mild cognitive impairment and various dementias. Psychiatry Research: Neuroimaging 2009;173:15-21.

82. Serra L, Cercignani M, Lenzi D, et al. Grey and White Matter Changes at Different 
Stages of Alzheimer's Disease. Journal of Alzheimer's Disease 2010;19:147159.

83. Wang H, Su MY. Regional Pattern of Increased Water Diffusivity in Hippocampus and Corpus Callosum in Mild Cognitive Impairment. Dementia and Geriatric Cognitive Disorders 2006;22:223-229.

84. Callen DJA, Black SE, Gao F, Caldwell CB, Szalai JP. Beyond the hippocampus: MRI volumetry confirms widespread limbic atrophy in AD. Neurology 2001;57:16691674.

85. Choo ILH, Lee DY, Oh JS, et al. Posterior cingulate cortex atrophy and regional cingulum disruption in mild cognitive impairment and Alzheimer's disease. Neurobiology of Aging 2010;31:772-779.

86. Jones BF, Barnes J, Uylings HBM, et al. Differential Regional Atrophy of the Cingulate Gyrus in Alzheimer Disease: A Volumetric MRI Study. Cerebral Cortex 2006;16:1701-1708.

87. Jacobs HIL, Van Boxtel MPJ, Jolles J, Verhey FRJ, Uylings HBM. Parietal cortex matters in Alzheimer's disease: An overview of structural, functional and metabolic findings. Neuroscience \& Biobehavioral Reviews 2011.

88. Chincarini A, Bosco P, Calvini P, et al. Local MRI analysis approach in the diagnosis of early and prodromal Alzheimer's disease. Neurolmage 2011;58:469-480.

89. Burgmans S, van Boxtel MPJ, Smeets F, et al. Prefrontal cortex atrophy predicts dementia over a six-year period. Neurobiology of Aging 2009;30:1413-1419. 


\section{SUPPLEMENTARY DATA}

Table S1. Measurement logistics MRI.

Rater time Computer calculation time

Manual hippocampal measurement 150

ICV measurement*

10

Segmentation with SIENAX**

$\begin{array}{ll}- & 75\end{array}$

LEAP hippocampal measurement

4

250

MTA-score

5

Lateral ventricle

30

Time in minutes per scan. *ICV measurement time should be added to manual hippocampal measurement. ${ }^{* *}$ Segmentation time should be added to time needed for lateral ventricle measurement. ICV: intracranial volume, LEAP: learning embeddings for altas propagation, MTA: medial temporal lobe atrophy. 
Table S2. Differences between $A D(A D+)$ and non-AD (AD-) in both cohorts.

\begin{tabular}{|c|c|c|c|c|}
\hline & \multicolumn{2}{|c|}{ Descripa } & \multicolumn{2}{|c|}{ VUmc } \\
\hline & $A D-(1)$ & $A D+(2)$ & $A D-(3)$ & $A D+(4)$ \\
\hline $\mathrm{N}$ & 126 & 30 & 111 & 61 \\
\hline Age & $69.4(55.2-89)$ & $74.1(63-90)^{* *}$ & $70.4(54-87)$ & $72(56-81)$ \\
\hline Female (\%) & 60.3 & 56.7 & 38.7 & 54.1 \\
\hline Years education & $8.4(3-18)^{\wedge \wedge}$ & $8.9(3-22)+$ & $11(6-18)$ & $11(6-17)$ \\
\hline $\begin{array}{l}\text { MMSE score } \\
\text { baseline }\end{array}$ & $27.5(18-30)$ & $26(21-29) * *$ & $27(19-30)$ & $26(18-30)^{* *}$ \\
\hline $\begin{array}{l}\text { Z-score wordlist - } \\
\text { delayed recal }\end{array}$ & $-1.07(-4.06 ; 1.96)$ & $\begin{array}{c}-1.9 \\
(-3.16 ;-0.21)^{* * *}\end{array}$ & $\begin{array}{c}-1.43 \\
(-3.16 ; 2.35)\end{array}$ & $\begin{array}{c}-1.94 \\
(-3.46 ;-0.05)^{*}\end{array}$ \\
\hline$\%$ ApoE 4 & 43.3 & 53.8 & 50 & 68.2 \\
\hline $\begin{array}{l}\text { Manual hippo- } \\
\text { campus }\end{array}$ & $\begin{array}{c}8107 \\
(5318-12882)^{\wedge \wedge}\end{array}$ & $\begin{array}{c}6891 \\
(5054-9149)^{* * *}\end{array}$ & $\begin{array}{c}7714 \\
(5454-10455)\end{array}$ & $\begin{array}{c}7003 \\
(4850-9892)^{* * *}\end{array}$ \\
\hline $\begin{array}{l}\text { Leap hippocam- } \\
\text { pus }\end{array}$ & $\begin{array}{c}6033 \\
(3684-8281)^{\wedge \wedge \wedge}\end{array}$ & $\begin{array}{c}5324 \\
(4297-7254)^{* * *}\end{array}$ & $\begin{array}{c}5601 \\
(4088-7061)\end{array}$ & $\begin{array}{c}5162 \\
(3327-6487) * * *\end{array}$ \\
\hline MTA-score & $2.5(0-6)^{\wedge \wedge \wedge}$ & $3.9(0-7)^{* * *+}$ & $1.8(0-6)$ & $3(0-8) * * *$ \\
\hline Lateral ventricle & $\begin{array}{c}51446 \\
(12128-126853)\end{array}$ & $\begin{array}{c}61652 \\
(25330-144640)\end{array}$ & $\begin{array}{c}53474 \\
(10786-162353)\end{array}$ & $\begin{array}{c}64470 \\
(8587-167163) *\end{array}$ \\
\hline
\end{tabular}

All volumetric measurements are corrected for intracranial volume.

AD: Alzheimer's disease, MMSE: Mini-Mental State Examination score, APOe4: apolipoprotein E4, LEAP: Learning Embeddings for Atlas Propagation, MTA: Medial temporal lobe atrophy. Differences between converters and non-converters in each separate cohort (group (1) vs group (2) and group (3) vs group (4): * $p<.05, * * p<.01, * * * p<.001$.

Differences between non-converters (group (1) vs group (3)): ${ }^{\wedge} p<.01,{ }^{\wedge \wedge \wedge} p<.001$. Differences between converters (group (2) vs group (4)): ${ }^{\dagger} p<.05$.

82 | Chapter 3: MTL measurements for AD prediction in $\mathrm{MCl}$ 
Table S3. Cut-off points.

\begin{tabular}{lllllll}
\hline & Youden & & \multicolumn{5}{c}{ Sensitivity $85 \%$} \\
& Descripa & VUmc & Both & Descripa & VUmc & Both \\
\hline Manual HC & $<7427$ & $<7590$ & $<7559$ & $<8647$ & $<8173$ & $<8379$ \\
LEAP HC & $<5374$ & $<5431$ & $<5374$ & $<6043$ & $<5794$ & $<5901$ \\
MTA-score (L+R) & $\geq 3$ & $\geq 3$ & $\geq 3$ & $\geq 3$ & $\geq 1$ & $\geq 2$ \\
Lateral ventricle & $\geq 58491$ & $\geq 45045$ & $\geq 58491$ & $\geq 31494$ & $\geq 38763$ & $\geq 34859$ \\
\hline
\end{tabular}

All volumetric measurements are corrected for intracranial volume and presented in $\mathrm{mm}^{3}$. HC: hippocampus, LEAP: Learning Embeddings for Atlas Propagation, MTA: Medial temporal lobe atrophy, L: left, R: right.

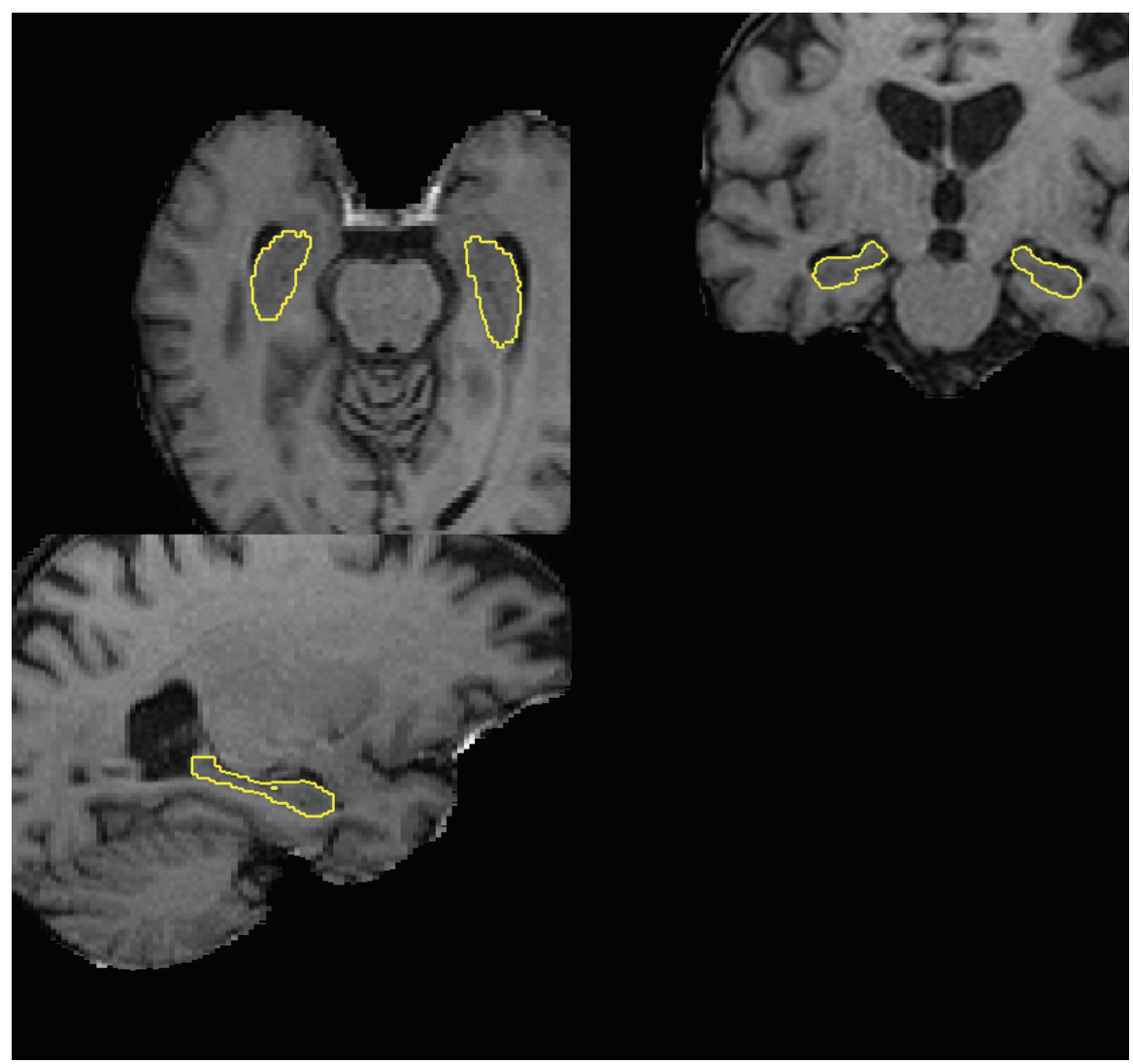

Fig. S1. Image of hippocampal LEAP segmentation. This figure shows an example of an image of hippocampal volume segmentation for horizontal, coronal, and sagittal sections using the atlas-based LEAP method. 


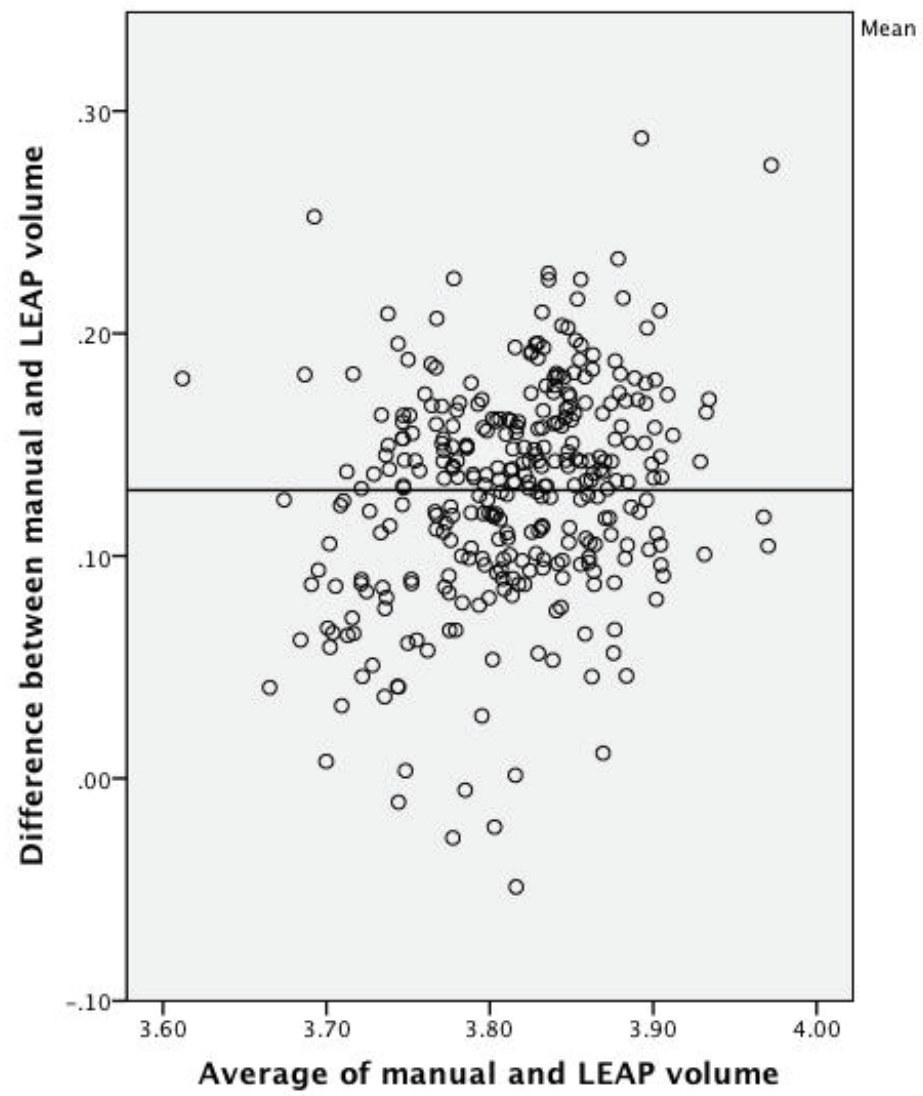

Fig. S2. Bland-Altman Plot displaying the agreement between both volumetric hippocampal measurements (values are log-transformed). Both measures are corrected for intracranial volume. 
Appendix S1. Scan parameters and MRI protocols used at each center.

\section{DESCRIPA}

\section{CENTER HUDDINGE}

Siemens Avanto 1.5 T, 21 slices, FOV 220 mm, FOV phase 87.1, distance factor 30, phase R>L, slice thickness 5.0 mm, TE: 96 ms, TR: 4000 ms, flip angle 150॰, number of averages 1 .

Siemens Symphony 1.5 T, 21 slices, FOV 220 mm, FOV phase 75.0, distance factor 30, phase R>L, slice thickness $5.0 \mathrm{~mm}$, TE: $99 \mathrm{~ms}$, TR: $4100 \mathrm{~ms}$, flip angle 150。, number of averages 2 .

\section{CENTER KUOPIO}

Siemens Vision 1.5 T, T1 3D-scan, MPRAGE OBL; COR>TRA, FOV 250, mat 256x256, 128 slices, TR $9.7 \mathrm{~ms}$, TE $4 \mathrm{~ms}$, Slice th $2.0 \mathrm{~mm}$, no slice gap, flip angle $12 \circ$

\section{CENTER MALMO}

Siemens Sonata 1.5 T, MPRAGE + Impr-cor, 144 slices, FOV $250 \mathrm{~mm}$, phase R>L, TR 1970, TE 3.93, distance factor 50, slice thickness $1.5 \mathrm{~mm}$, flip angle 15.

\section{CENTER MUNICH}

Siemens Magnetom Vision; 1.5 T; MPRAGE; Slice thickness $1.05 \mathrm{~mm}$, TR 11.4, TE 4.4, TI 300; FOV 256*256; Flip angle 8 graden; number of averages 1.

\section{CENTER THESSALONIKI}

Siemens Expert Plus unit 1.0 T, 3D-MPR: 15 (TR) ,7 TE ,8 FLIP ANG., 250 (Slabth), 1,49Ef thick, 168 Partitions, 250 FOV,256×192 Matrix, 1 Aquis. ,ACQ TIME 10,21min

\section{CENTER BRESCIA}

Philips Gyroscan PG 1.0 Tesla: sagittal 3D T1 scan: $T R=20 \mathrm{~ms}$, TE $=5 \mathrm{~ms}$, flip angle= $30^{\circ}$, field of view $=220 \mathrm{~mm}$, acquisition matrix $256 \times 256$, number of slices $=100 / 130$, slice thickness $1,3 \mathrm{~mm}$. 


\section{CENTER GENOA}

Philips Intera 1.5 Tesla: Sagittal MP RAGE : t1w/3d/tfe, TR 8.5, TE 3.9, Flip 8, thickness 1 , no overcontiguous, no gap, matrix $100 \% 256$, fov 256 , reconstructed voxel size 1.00/1.00/1.00, rfov 100\%, T1 t1w/se, TR 580, TE 15, flip 69, slices 22, thick 5mm, matrix $80 \% 240$, fov 240 , rfov $75 \%$, pre (tra)- and postGD-GDPA (tra, sag, cor)

\section{CENTER MAASTRICHT}

Philip NT, 1.5 T Gyroscan: T1-weighted images obtained in the coronal plane using a 3D-gradient fast field echo (FFE) sequence. $\mathrm{TR}=35 \mathrm{~ms}, \mathrm{TE}=7 \mathrm{~ms}, \mathrm{FA}=35, \mathrm{FOV}=240$ $\mathrm{mm}$, slice thickness $=1.5 \mathrm{~mm}$, matrix size $=256 \times 256$, voxelsize $=0.94 \mathrm{~mm} \times 0.94 \mathrm{~mm}$ $x 1.5 \mathrm{~mm}$.

\section{CENTER MANNHEIM}

Siemens Medical solution Magnetom, Vision plus 1.5 Tesla: T1 MPR 30, TR=11.4 $\mathrm{ms}, \mathrm{TE}=4.4 \mathrm{~ms}$, flipangle $=15, \mathrm{FoV}=256 \mathrm{~mm}$, format $8 / 8$, slices=162, no gap.

CENTER VUmc (VUmc data from DESCRIPA + an additional sample)

Siemens Magnetom Impact Expert 1.0 T, 3D scan, 168 slices, FOV 250 mm, matrix 256 × 256; slice thickness 1.5 mm, TE: 7 ms, TR: 15 ms, TI 300 ms, flip angle 15.

\section{REFERENCES}

Andreasen, N., Hesse, C., Davidsson, P., Minthon, L., Wallin, A., Winblad, B., et al. (1999). Cerebrospinal Fluid \{beta\}-Amyloid(1-42) in Alzheimer Disease: Differences Between Early- and Late-Onset Alzheimer Disease and Stability During the Course of Disease. Archives of Neurology, 56(6), 673-680. 



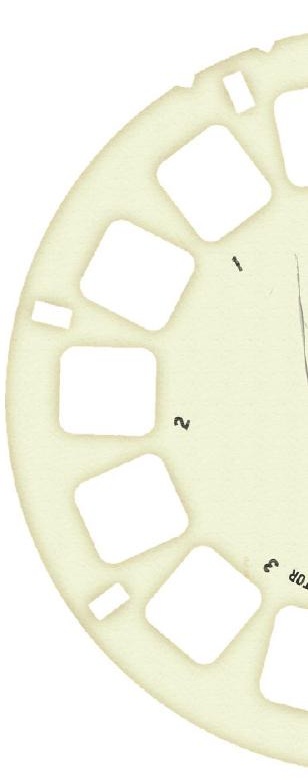




\section{Chapter 4}

Sensitivity of different MRI-techniques

to assess gray matter atrophy patterns in

Alzheimer's disease is region-specific

Current Alzheimer Research, 2013 Nov;10(9):940-51

$\uparrow$

c)

N

Lies Clerx, Heidi I.L. Jacobs, Saartje Burgmans, Ed H.B.M. Gronenschild, Harry B.M. Uylings, Carmen Echávarri, Pieter-Jelle Visser, Frans.R.J. Verhey \& Pauline Aalten 


\section{ABSTRACT}

The present study compares four different structural magnetic resonance imaging techniques used to measure gray matter (GM) atrophy in Alzheimer's disease (AD): manual and automated volumetry, cortical thickness (CT) and voxel-based morphometry (VBM). These techniques are used interchangeably in AD research and thus far it is unclear which technique is superior in detecting abnormalities early in the disease process. 18 healthy participants without any memory impairment, 18 patients with $\mathrm{MCl}$, and 17 patients with mild $A D$ were included and betweengroup differences were investigated in $A D$ signature regions (areas in the prefrontal cortex (PFC), medial temporal lobe (MTL) and posterior parietal cortex (PPC)). Both manual volumetric measurements and VBM were able to detect GM atrophy in the early stages (differentiation controls and $\mathrm{MCl}$ ), mainly in the MTL. In the early phase, automated volumetric measurements showed GM differences in the PPC but not in the MTL. In our sample, CT measurements were not sensitive for group differences in the early stages. PFC regions showed abnormalities in the later stages (controls vs $A D$ ) when manual volumetric measurements or VBM are employed. Manual volumetric measurements together with VBM are preferred techniques for assessing GM differences showing abnormalities in most of the investigated regions, with a predominance of the MTL in the early phase. Automated FreeSurfer volumetric measurements show similar performances in the early phase, displaying group differences in the PPC but not in MTL regions. Measurements of CT are less sensitive in the $\mathrm{MCl}$ stage and it's sensitivity is restricted to the MTL and PPC regions in later stages of the disease (AD). 


\section{INTRODUCTION}

Since the prevalence of Alzheimer's disease (AD) is expected to increase rapidly in the following decades, early detection and intervention in persons who are at risk for developing $A D$ is an important public health goal. Clinical and pathological evidence indicates that older individuals who have significant memory impairment but are not demented may be in a transitional phase between normal aging and $A D$, a state often referred to as as 'mild cognitive impairment' (MCI) [1]. A large part of the patients with $\mathrm{MCl}$ have a high likelihood of progressing to probable $A D$ within a few years [2-4]. Brain imaging can aid the early diagnostic process, ultimately by detecting neurodegenerative abnormalities at an earlier stage than the standard neurological examination or before objective signs are present $[5,6]$. Imaging can furthermore help to select $\mathrm{MCl}$-subjects for AD-trials by detecting differences in an early stage and is able to monitor disease progression.Within the domain of magnetic resonance imaging (MRI), the major focus lies on manual segmentation of structures known to be involved in dementia, especially the parahippocampal gyrus and the hippocampus [7-9].

The present study compares four different structural MRI techniques used to assess gray matter (GM) atrophy in AD. Manual volumetry, still considered gold standard [10-12], is however time-consuming, making it difficult to implement in routine clinical practice or for large-scale research use. To overcome the disadvantages of manual volumetry, techniques to perform automated (whole brain) analyses have been designed, e.g. Voxel based morphometry (VBM), automated segmentation methods (e.g. FreeSurfer) and cortical thickness (CT). Automated measurements are widely implemented and fast, but may underestimate atrophy status and thus lead to false negatives results [13]. CT analysis in AD patients has revealed cortical thinning in several brain regions known to be affected in $A D$ [14-16] and this thinning has furthermore shown to be related to the severity of $A D$, even in early stages [17]. The main disadvantage of the method is its inaccuracy in medial cortical areas (e.g. parahippocampal gyrus) and the impossibility to determine cortical thickness in the hippocampal region.

Another widely used technique to study brain atrophy in-vivo is voxel-based morphometry (VBM), a method to calculate voxel-wise gray matter concentration of 
brain areas based on a whole-brain analysis $[18,19]$. Volume changes of $G M$, white matter (WM) and cerebrospinal fluid (CSF) are examined separately. The automated computational procedures of VBM are unaffected by inter-rater differences and anatomical boundary shifts are minimized by the utilization of statistical maps and segmentation techniques [20]. VBM can be applied as a first-pass strategy, to generate hypotheses [21]. Disadvantages of VBM can be found in the pre-processing phase, when warping and smoothing of MRI data is applied. Warping algorithms may not always succeed in matching the anatomical characteristics across participants and smoothing furthermore reduces the individual variability of gyrus and sulcus features [22]. Especially when degenerated brains are studied, these steps might induce both type I and II errors in the outcome. VBM furthermore does not provide information of brain atrophy at a single-subject level, it only permits group-level analysis, limiting its diagnostic employability in the clinical setting.

The four techniques mentioned above are used interchangeably in $A D$ research and thus far it is unclear which technique is superior in detecting abnormalities early in the disease process. Since manual volumetric measurements of the medial temporal lobe (MTL) are hard to implement in everyday clinical practice, possible alternatives to measure GM atrophy in AD are evaluated. It is furthermore known that AD pathology is not restricted to the MTL lobe and that prefrontal [23] and posterior parietal regions [24] also show abnormalities early in the disease process. In this study, regions were selected based on their relevance for AD.

This is, to our knowledge, the first study directly comparing four techniques which measure gray matter atrophy in a group of healthy controls, patients with $\mathrm{MCl}$ and patients suffering from mild AD. We evaluated which technique is superior in detecting abnormalities early in the disease process. In addition, we investigated whether atrophy of prefrontal cortex (PFC), posterior parietal cortex (PPC) or medial temporal lobe (MTL) regions is most indicative of (preclinical) AD.

\section{MATERIALS AND METHODS \\ Subjects}

Three groups of older male participants were included: 20 healthy participants 
without any objective memory impairment (controls), 20 patients with amnestic $\mathrm{MCl}$, and 20 patients with mild AD. Controls were recruited by means of an advertisement in local newspapers. An extensive neuropsychological test battery was administered and subjects were included when their performance did not deviate from normal on the Verbal Learning Test $[25,26]$. Patients with $\mathrm{MCl}$ and mild $A D$ were recruited from the Memory Clinic of the Maastricht University Hospital. Diagnosis was made according to the Petersen criteria for $\mathrm{MCl}$ (with at least an impairment in the memory domain) [27, 28], and the DSM-IV [29] and NINCDSADRDA criteria for AD [30]. Diagnosis was based on medical history, co-morbidity, course, and MRI scanning. The MRI scan was only acquired to exclude neurosurgical lesions vascular pathology; levels of atrophy were not used for the diagnosis of $\mathrm{MCl}$ and AD.

Exclusion criteria were: MRI-contraindication, abuse of alcohol and drugs, other past or present psychiatric or neurological diseases or serious system diseases, and structural abnormalities in the brain that could account for the cognitive decline. Two participants in the control group were excluded because a brain infarct was detected on the MRI scans. Two participants in the $\mathrm{MCl}$ group, and three in the AD group were excluded because their MRI images showed motion artefacts. The study was approved by the local Medical Ethics Committee of the Maastricht University Medical Centre. Written informed consent was obtained from all participants and from the primary caregiver of the AD patients in accordance with the committee's guidelines and with the Declaration of Helsinki [31].

\section{Image acquisition and analysis}

MRI scans were acquired with a 3 Tesla Gyroscan NT MRI scanner (Philips, Best, The Netherlands). Structural T1 images were acquired in the sagittal plane using an MPRAGE sequence $\left(T R=8, T E=3.7 \mathrm{msec}, F A=8^{\circ}, F O V=240 \times 240\right.$, matrix size $=240$ $x 240$, number of slices $=180$ ). Regions of interest were selected in the prefrontal cortex (inferior prefrontal cortex, orbitofrontal cortex), posterior parietal cortex (precuneus, posterior cingulate cortex) and medial temporal lobe (hippocampus, parahippocampal gyrus). For each technique, left and right side of each selected region were analyzed separately. 


\section{Manual volumetry}

For the manual tracing we used GIANT (General Image Analysis Tools; [32]), a customized software program which allows tracing of regions of interest (ROI) in a triplanar and rotatable 3D surface-rendered view, and calculation of GM volumes of interest. Boundaries of selected frontal and temporal structures were set according to criteria described in a previous publication [33] (Supplementary Table S1). Boundaries of the posterior cingulate and precuneus cortex were adapted from Jones et al. [34] and Ryu et al. [35] respectively (Supplementary Table S1).

Both raters (LC, CE) were blind to the demographic and cognitive characteristics of the participants. Intra-rater reliability was determined by the Intraclass Correlation Coefficient (ICC) [36]. To account for age-related cortical shrinkage we measured the intracranial volumes (ICV) with the FSL Brain Extraction Tool [37]. Our analyses were corrected for ICV, which partials out individual variability in maximal total brain volume achieved in adulthood independent from possible brain atrophy at later ages (Supplementary Table 1). To control for Type I errors, we performed the false discovery rate (FDR) controlling procedure, a correction for multiple comparisons [38]. This method differs from the classical Bonferonni approach in that it is less stringent and as such also reduces the increased probability of making Type II errors.

\section{Automated volumetry}

In accordance with Gronenschild et al. [39], a Macintosh with OSX 10.5.8. was used for analysis. Cortical reconstruction and volumetric segmentation was performed with FreeSurfer version 4.5.0, which is freely available (http://surfer.nmr.mgh. harvard.edu). In this approach, brain areas are segmented using a nonlinear template matching [40]. After linearly registering the test image to the template, the algorithm estimates the nonlinear transformation between a given MRI and a probabilistic atlas of the selected brain structure constructed from a cohort of 14 young and middle-aged subjects using a maximum likelihood criterion. Probabilistic labels are warped back to the individual MRI using the inverse of this transform. The final segmentation is accomplished by maximizing the a posteriori probability in the Bayes formula at each voxel. Voxel-wise probabilistic labels and their predicted image intensities serve as the prior term, while the intensity similarity between the target image and the template serves as the likelihood term. 
In this study, FreeSurfer voxel volumes were used (not the tabulated volumes corrected for partial volume effects) in order to obtain a proper comparison with the manual volumetric measurement. The borders of the FreeSurfer ROI of the isthmus fits best with our definition of the posterior cingulate cortex as adopted for the manual segmentation and were subsequently used. The posterior cingulate cortex $\mathrm{ROI}$ included in the FreeSurfer automated measurement is more rostral compared to our definition.

\section{Cortical thickness (CT)}

The FreeSurfer CT pipeline has been described and validated in previous publications (Dale et al., 1999; Fischl et al., 1999; Fischl and Dale, 2000; Han et al., 2006). To summarize, processing involves intensity normalisation, registration to Talairach space, skull stripping, segmentation of WM, tesselation of the WM boundary, smoothing of the tesselated surface and automatic topology correction. The tesselated surface is used as the starting point for a deformable surface algorithm to find the WM and then the pial boundary. For each point on the tesselated WM surface, the CT is calculated as the average of the distance from the WM surface to the closest point on the pial surface and from that point back to the closest point on the WM surface [41]. The cortex of the brain was automatically subdivided into gyral-based regions of interest (ROIs) [42]. To accomplish this, a registration procedure was used that aligns the cortical folding patterns and probabilistically assigns every point on the cortical surface to one of the 32 ROIs. For the purposes of this study, we focused on 6 ROIs bilaterally. For each ROI the mean cortical thickness was extracted for subsequent statistical analysis.

For our second approach, a vertexwise analysis, we mapped the thickness measures on the inflated surface of each participant's reconstructed brain. This allows visualization across the surface without interference from cortical folding. Maps were smoothed using a circularly symmetric Gaussian kernel with a full width half maximum (FWHM) of $20 \mathrm{~mm}$ and averaged across participants using a non-rigid high-dimensional spherical method to align cortical folding patterns to a template supplied by FreeSurfer. This procedure provided accurate matching of morphologically homologous cortical locations among participants on the basis of each individual's anatomy while minimizing metric distortion, resulting in a mean measure of cortical thickness at each vertex on the reconstructed surface.

Statistical comparisons between the surface maps were generated by computing a 
general linear model of the group effects (corrected for age centered) on thickness at each vertex in the cortical mantle. Maps were created using a statistical threshold of $p=0.05$. A cluster-wise procedure was performed to correct for multiple comparisons. This method utilizes a simulation to get a measure of the distribution of the maximum cluster size under the null hypothesis. Z-maps are synthesized and smoothed using a residual FWHM, and then thresholded at $p=0.05$. Next, areas of maximum clusters are recorded, under these specifications, and the procedure is repeated for 5000 iterations. Once the distributions of the maximum cluster size are obtained, correction for multiple comparisons is performed by finding clusters in the statistical maps using the same threshold as was given in the simulation procedure. For each cluster, the $p$ value is the probability of seeing a maximum cluster of that size, or larger, during the simulation. Clusters remaining in similar areas of significance as in the original cortical thickness maps would indicate that the result is not likely due to chance. For each cluster, maximum, minimum, mean and standard deviation of the $p$-values were extracted.

\section{Voxel-based morphometry (VBM)}

Structural data was analyzed with FSL-VBM, a voxel-based morphometry style analysis carried out with FSL tools [43]. First, structural images were brain-extracted using BET [37]. Next, tissue-type segmentation was carried out using FAST4 [44]. The resulting GM partial volume images were then aligned to MNI152 standard space using the affine registration tool FLIRT $[45,46]$ followed by nonlinear registration using FNIRT $[47,48]$, which uses a b-spline representation of the registration warp field [49]. The resulting images were averaged to create a studyspecific template, to which the native gray matter images were then non-linearly reregistered. The registered partial volume images were then modulated (to correct for local expansion or contraction) by dividing by the Jacobian of the warp field. The modulated segmentated images were then smoothed with an isotropic Gaussian kernel with a sigma of $2 \mathrm{~mm}$. Finally, voxelwise general linear modeling (GLM) was applied using permutationbased nonparametric testing (5000 permutations). [50] ROI's for displaying significant volume loss were selected based on the HarvardOxford cortical and subcortical atlas, which is also integrated in FSLview for inspection and identification. The following regions were selected: precuneus, hippocampus, parahippocampal gyrus, posterior cingulate gyrus, inferior frontal gyrus (sum of pars opercularis and pars triangularis) and frontal orbital cortex. 


\section{Statistical analysis}

Statistical analyses were performed with the Statistical Package for the Social Sciences (SPSS, Chicago, IL, USA) version 19.0. Demographical and cognitive group differences were compared with ANOVA. The ROI-based measures (manual volumetric measurement, FreeSurfer automated measurement and mean CT) were compared between the 3 groups by means of a pairwise ANCOVA for each separate technique (manual volumetric measurement, FreeSurfer automated measurement and CT-ROI measurement), with volume as dependent variable, group as independent variable (fixed factor), and intracranial volume (ICV) and centered age as covariates. For the vertex-wise CT-analysis, statistical analysis was carried out by means of FreeSurfer (see above, paragraph 2.2.3). For VBM, voxelwise GLM was applied using permutation-based non-parametric testing, including a correction for multiple comparisons across space. Corrected $p$-values are stated in the result section $\left(p_{\text {corr }}\right)$.

\section{RESULTS}

\section{Subject characteristics}

The three groups significantly differed with respect to age ( $M C l \angle A D, p=0.03$; $\mathrm{CON}<\mathrm{AD}, p=0.02$ ), Mini-Mental state examination (MMSE) score ( $\mathrm{CON}>\mathrm{MCl}, p=0.03$; $\mathrm{MCl}>\mathrm{AD}, p<.001 ; \mathrm{CON}>\mathrm{AD}, p<.001)$ and score on the delayed recall task (CON>MCl, $p<.001 ; \mathrm{MCl}>\mathrm{AD}, p<.05 ; \mathrm{CON}>\mathrm{AD}, p<.001)$, but not with respect to educational level. Subject characteristics are shown in table 1 and volumetric comparisons between the three groups are shown in supplementary table S2 (manual segmentation) and supplementary table S3 (automated segmentation).

\section{Group differences}

\section{Manual volumetric measurement}

Manual volumetric measurements of the right parahippocampal gyrus ( $\mathrm{PhG}$ ) were able to differentiate between controls and $\mathrm{MCl}$ patients ( $\left.\mathrm{F}=15.93, p_{\text {corr }}<.001\right)$. In later stages of the disease (controls versus $A D$ ) differentiation was possible in the following structures: left hippocampus $\left(F=5.14, p_{\text {corr }}<.05\right)$, right hippocampus $\left(\mathrm{F}=6.86, p_{\text {corr }}<.05\right)$, left PhG $\left(\mathrm{F}=29.87, p_{\text {corr }}<.001\right)$, right $\mathrm{PhG}\left(\mathrm{F}=21.21, p_{\text {corr }}<.001\right)$, left orbitofrontal cortex (OFC) ( $\left.F=20.24, p_{\text {corr }}<.001\right)$, right orbitofrontal cortex (OFC) $\left(\mathrm{F}=11.28, p_{\text {corr }}<.01\right)$, left posterior cingulate cortex $(\mathrm{PCC})\left(\mathrm{F}=4.90, p_{\text {corr }}<.05\right)$, left 
precuneus $(\mathrm{PC})\left(\mathrm{F}=7.50, p_{\text {corr }}<.01\right)$ and right $\mathrm{PC}\left(\mathrm{F}=15.88, p_{\text {corr }}<.001\right)$. Discrimination between early and later stages of the disease ( $M C l$ versus $A D)$ was possible by manual measurements of the left PhG $\left(\mathrm{F}=17.20, p_{\text {corr }}<.001\right)$, left inferior prefrontal cortex (IPFC) (F=11.11, $\left.p_{\text {corr }}<.01\right)$, left OFC ( $\left.F=10.66, p_{\text {corr }}<.01\right)$ and right PC (F=6.48, $\left.p_{\text {corr }}<.01\right)$ (Table 2).

Table 1. Subject characteristics

\begin{tabular}{lccc}
\hline & Con & $\mathrm{MCl}$ & $\mathrm{AD}$ \\
\hline $\mathrm{N}$ & 18 & 18 & 17 \\
Age & $64.56(3.4)$ & $65.11(4.5)$ & $70.59(9.1)$ \\
Educational level & $4(1.4)$ & $4(1.8)$ & $4(1.9)$ \\
MMSE score & $28.89(0.9)$ & $27.61(2.3)$ & $21.18(3.9)$ \\
15 WLT learning & $37.50(7.6)$ & $26.06(9.8)$ & $23.47(11.7)$ \\
15 WLT memory & $8.56(1.9)$ & $3.67(2.8)$ & $1.73(2.4)$ \\
Fluency animals & $23(5.3)$ & $21(5.4)$ & $13.93(4.7)$ \\
Manual hippocampus & $4656(308) /$ & $4410(482) /$ & $3883(817) /$ \\
volume L/R (mm $\left.{ }^{3}\right)$ & $4758(637)$ & $4308(796)$ & $3807(876)$ \\
ICV (ml) & $1492(100)$ & $1539(121)$ & $1574(125)$ \\
\hline
\end{tabular}

All volumetric measurements are corrected for intracranial volume. Values are mean (sd). MMSE: Mini-Mental state examination; WLT: wordlist; Con: controls; $\mathrm{MCl}$ : mild cognitive impairment; AD: Alzheimer's disease.

\section{Automated volumetric measurement}

FreeSurfer automated volumetric measurements were able to differentiate between controls and $\mathrm{MCl}$-patients in the left PCC $\left(\mathrm{F}=7.82, p_{\text {corr }}<.01\right)$ and right PC $\left(F=6.78, p_{\text {corr }}<.05\right)$. As the disease progresses, differences between controls and $A D$ were most notably in the left hippocampus $\left(\mathrm{F}=11.54, p_{\text {corr }}<.01\right)$, right hippocampus $\left(\mathrm{F}=13.35, p_{\text {corr }}<.001\right)$, left PCC $\left(\mathrm{F}=14.04, p_{\text {corr }}<.001\right)$, right PCC $\left(\mathrm{F}=16.34, p_{\text {corr }}<.001\right)$ and right $\mathrm{PC}\left(\mathrm{F}=12.69, p_{\text {corr }}<.001\right)$ regions. $\mathrm{MCl}$ and $\mathrm{AD}$ patients could be distinguished by automated measurements of the right PCC $\left(\mathrm{F}=9.85, p_{\text {corr }}<.01\right)$. 


\section{Cortical thickness}

\section{Quantification of cortical thinning by atlas-based ROI-analysis}

Differences in CT were only present in the comparison between controls and ADpatients in the right PCC ( $\left.\mathrm{F}=9.27, p_{\text {corr }}<.01\right)$ and right ERC (Table 2$)$.

\section{Vertex-wise group comparison with clusterbased correction}

Vertex-wise comparison of $C T$ between controls and $A D$ revealed one cluster in the left hemisphere and three clusters in the right hemisphere that demonstrated significantly reduced cortical thickness in AD (Figure 1, Table 3). These clusters mainly comprised temporal and parietal cortical areas. Two clusters in the left hemisphere, entirely situated in the frontal lobe, showed increased cortical thickness between controls and $A D$. For the comparison between $M C l$ and $A D$, thinning was found in $A D$ patients compared to $\mathrm{MCl}$ in the left hemisphere in parietal and temporal regions. In the right hemisphere thinning was found in parietal, frontal and temporal regions (Figure 2, Table 3).

\section{VBM}

The comparison between controls and $\mathrm{MCl}$ or $\mathrm{AD}$ showed several regions of significant GM loss compared with controls ( $p<0.05$, TFCE-corrected). VBM measurements of the left and right hippocampus were able to differentiate between controls and $\mathrm{MCl}$ patients ( $p<.05$ and $p<.001$ respectively, TFCE corrected). In later stages (controls versus $A D)$, differentiation was possible in the following structures: left $(p<.001$, TFCE corrected) and right ( $p<.001$, TFCE corrected) hippocampus, left $(p<.001$, TFCE corrected) and right PhG $(p<.001$, TFCE corrected $)$, right IPFC $(p<.001$, TFCE corrected), left ( $p<.01$, TFCE corrected) and right OFC ( $p<.01$, TFCE corrected), left $(p<.001$, TFCE corrected) and right PCC $(p<.001$, TFCE corrected $)$ and left $(p<.01$, TFCE corrected) and right PC ( $p<.001$, TFCE corrected). Discrimination between early and later stages of the disease was found for the left ( $p<.001$, TFCE corrected) and right ( $p<.001$, TFCE corrected) hippocampus, left ( $p<.001$, TFCE corrected) and right PhG ( $p<.001$, TFCE corrected), left ( $p<.01$, TFCE corrected) and right IPFC ( $p$ $<.01$, TFCE corrected), left $(p<.01$, TFCE corrected) and right OFC $(p<.001$, TFCE corrected), right PCC ( $p<.05$, TFCE corrected), and left ( $(p<.01$, TFCE corrected) and right PC ( $p<.05$, TFCE corrected)) (Table 4$)$. 


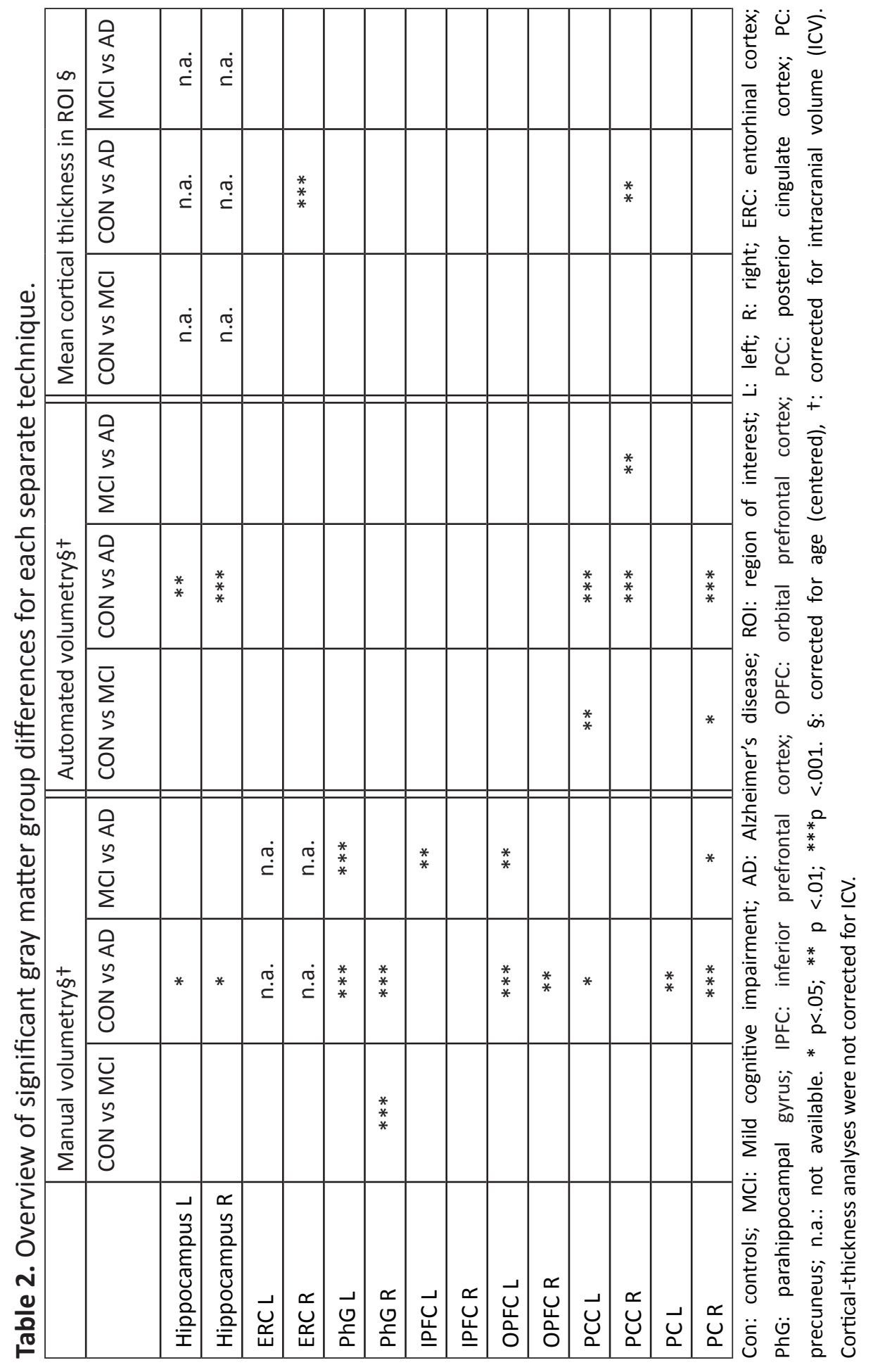




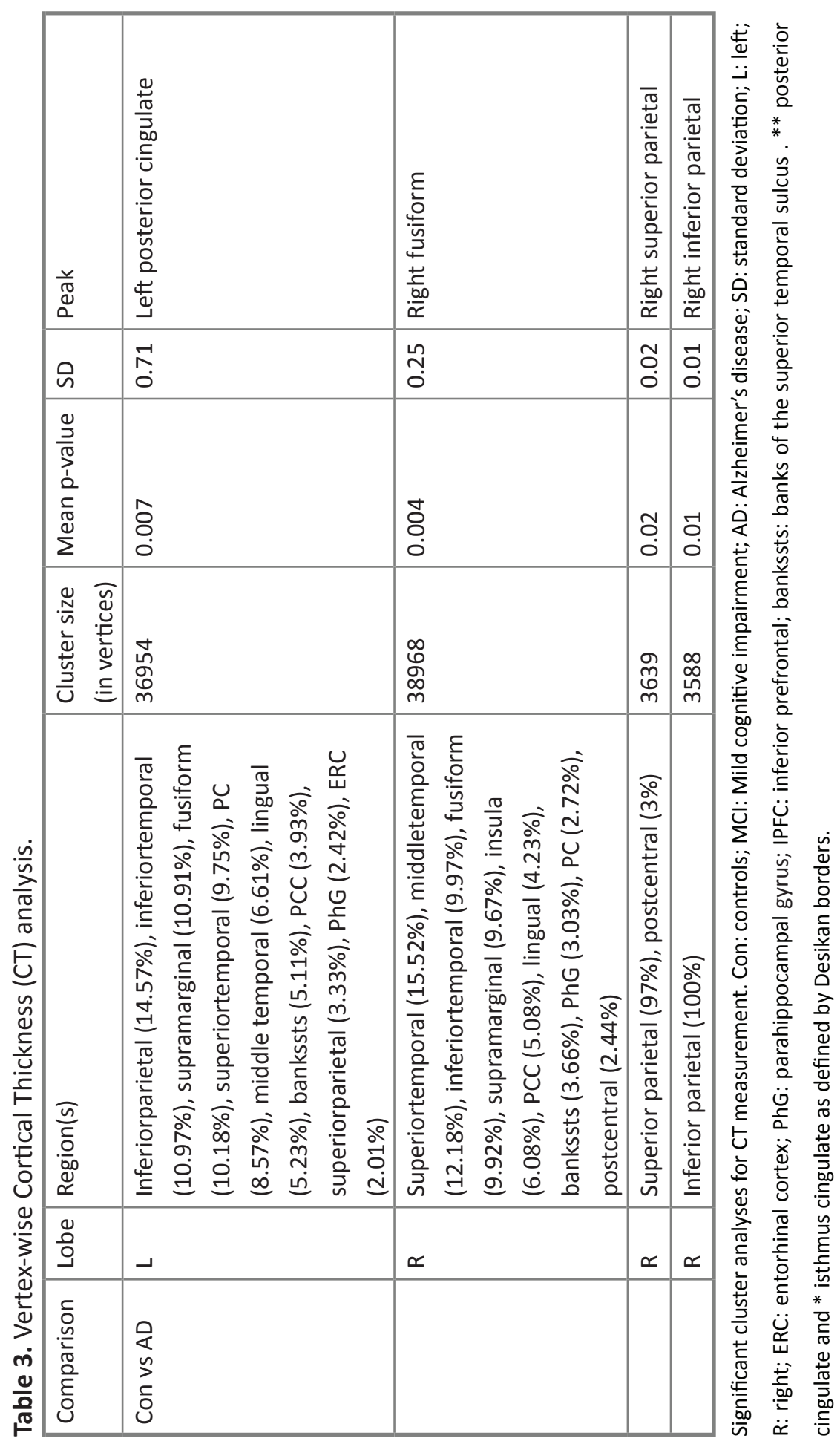




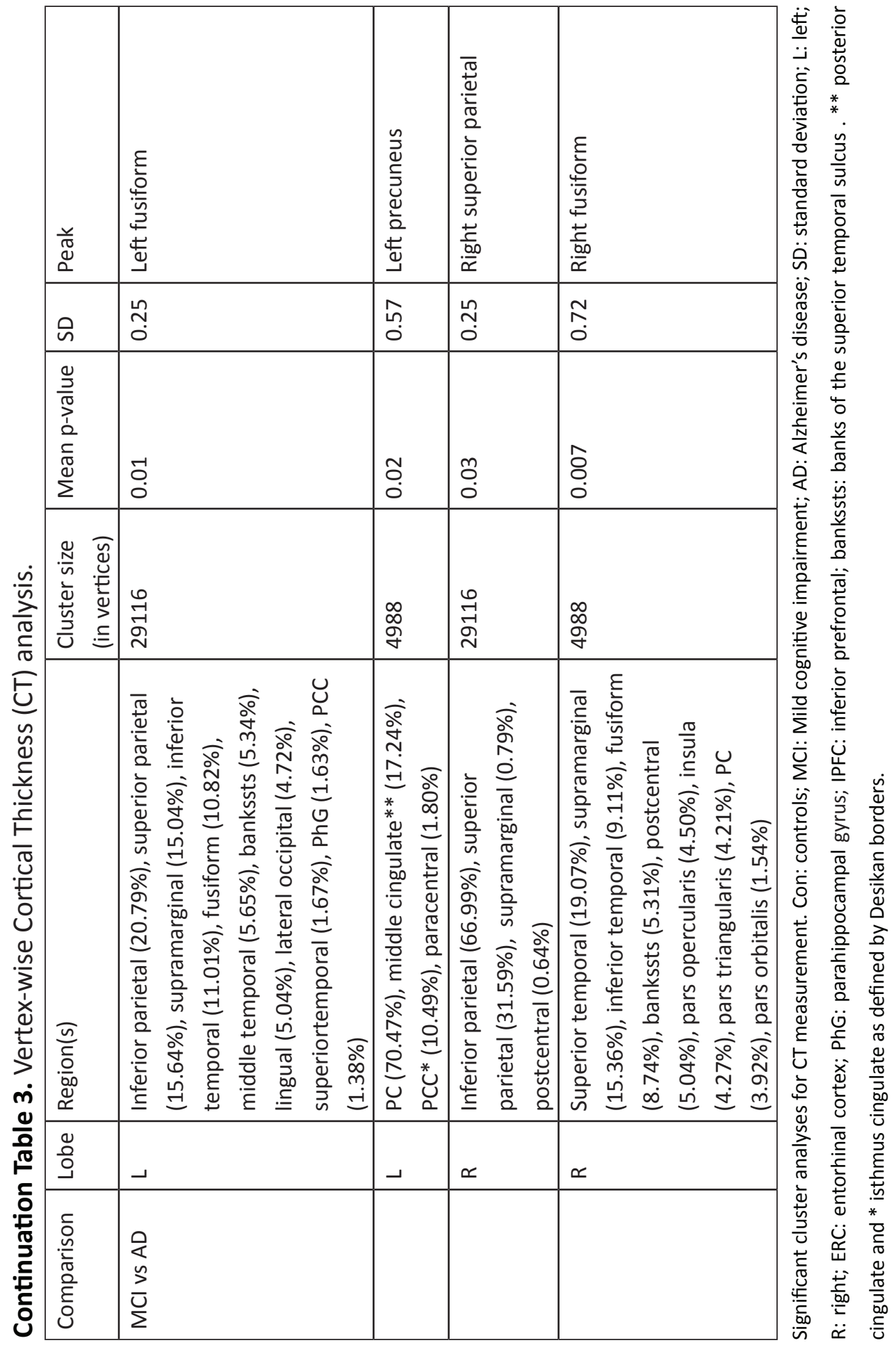



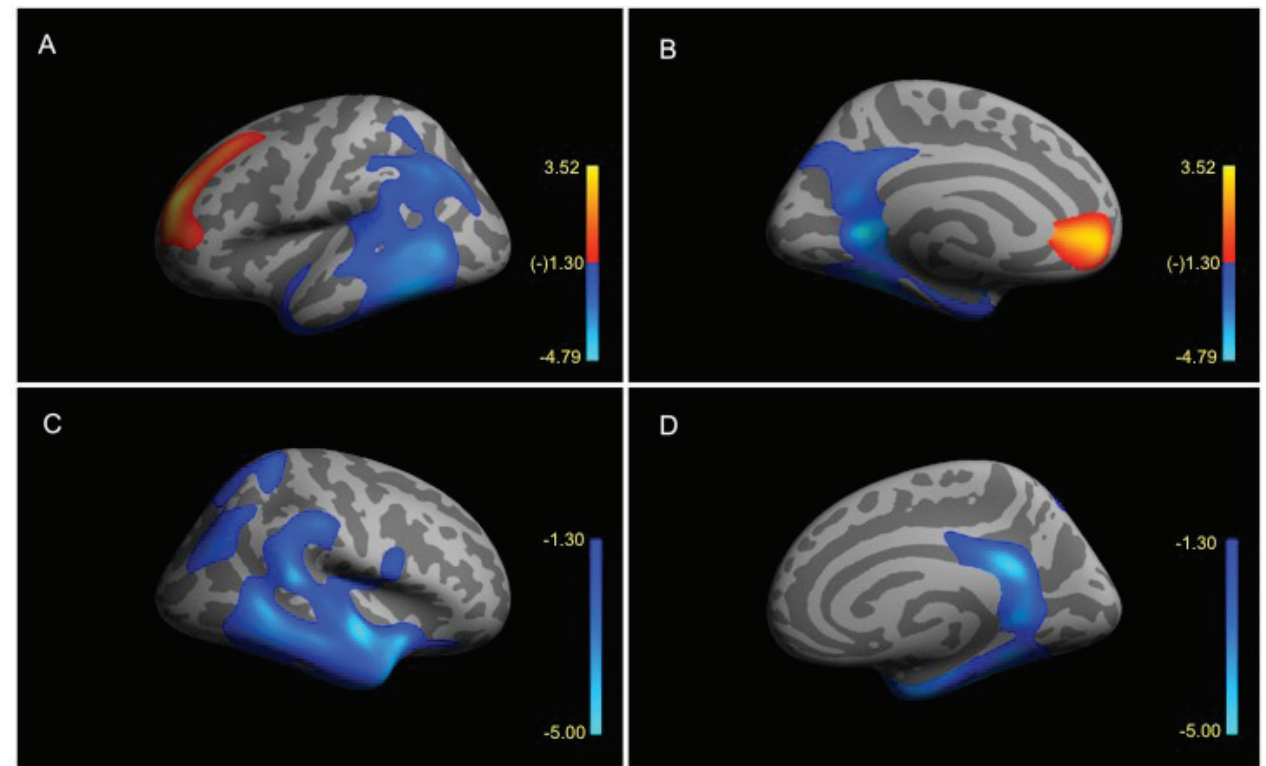

Figure 1. Cortical-thickness (CT) analyses for the differentiation between controls and AD-patients as measured by FreeSurfer. A. Left hemisphere lateral view, B. Left hemisphere medial view, $C$. Right hemisphere lateral view and D. Right hemisphere medial view. Vertex-wise comparison showed one cluster in the left hemisphere and three clusters in the right hemisphere displaying significantly reduced cortical thickness in AD (A, B, C and D: temporal and parietal cortical areas; blue) compared to controls. Two clusters in the left hemisphere, entirely situated in the frontal lobe showed increased cortical thickness between controls and AD ( $A$ and $B$ : red-yellow). 


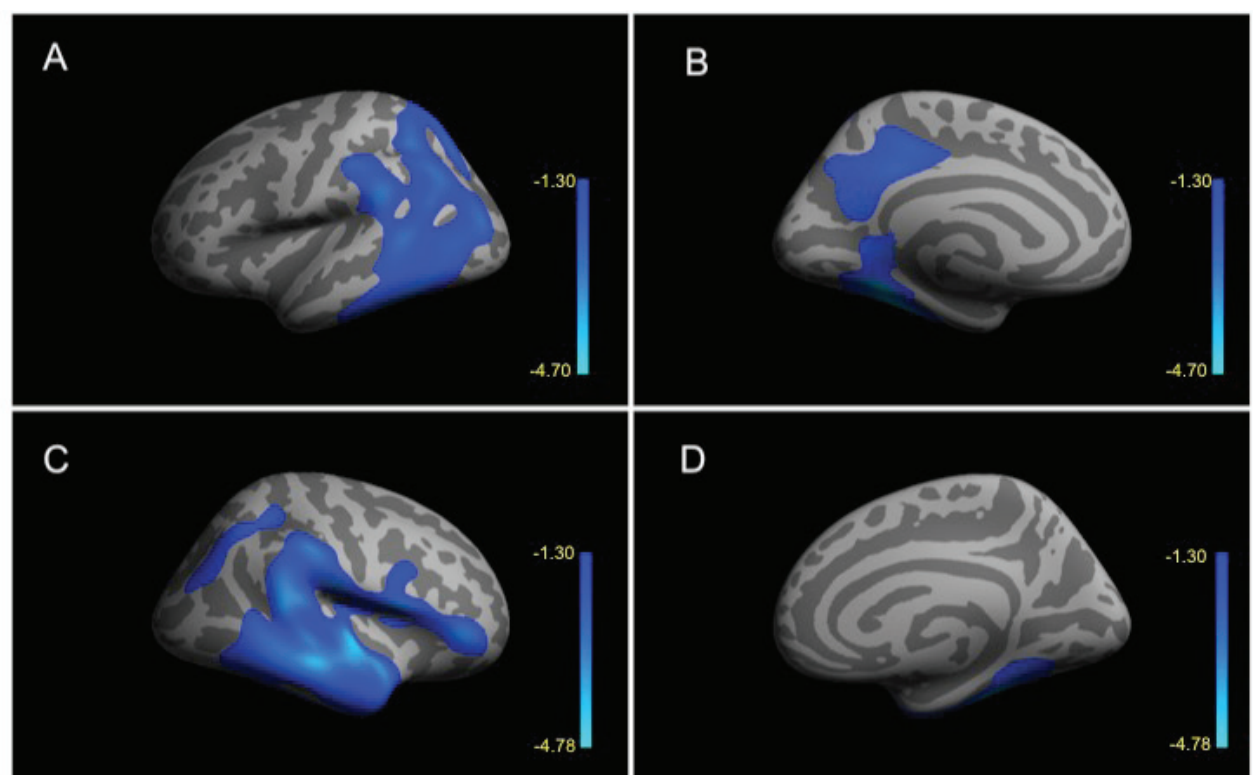

Figure 2. Cortical-thickness analyses for the differentiation between $\mathrm{MCl}$ and AD-patients as measured by FreeSurfer. A. Left hemisphere lateral view, B. Left hemisphere medial view, $C$. Right hemisphere lateral view and D. Right hemisphere medial view. Vertex-wise comparison showed thinning patterns in AD patients compared to $\mathrm{MCl}$ in the left hemisphere in parietal and temporal regions ( $A$ and $B$ ). In the right hemisphere thinning was found in parietal, frontal and temporal regions (C and D). 


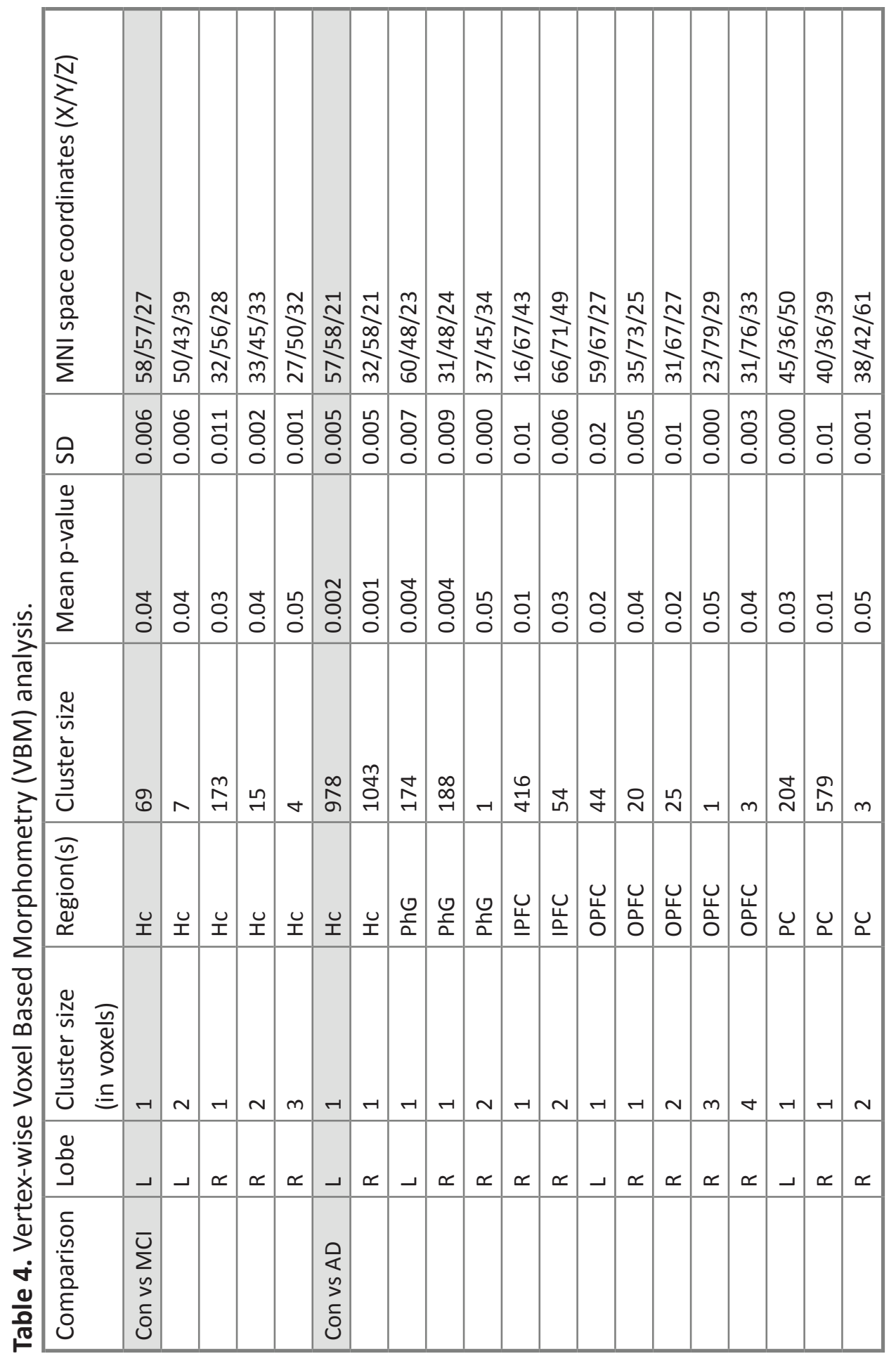




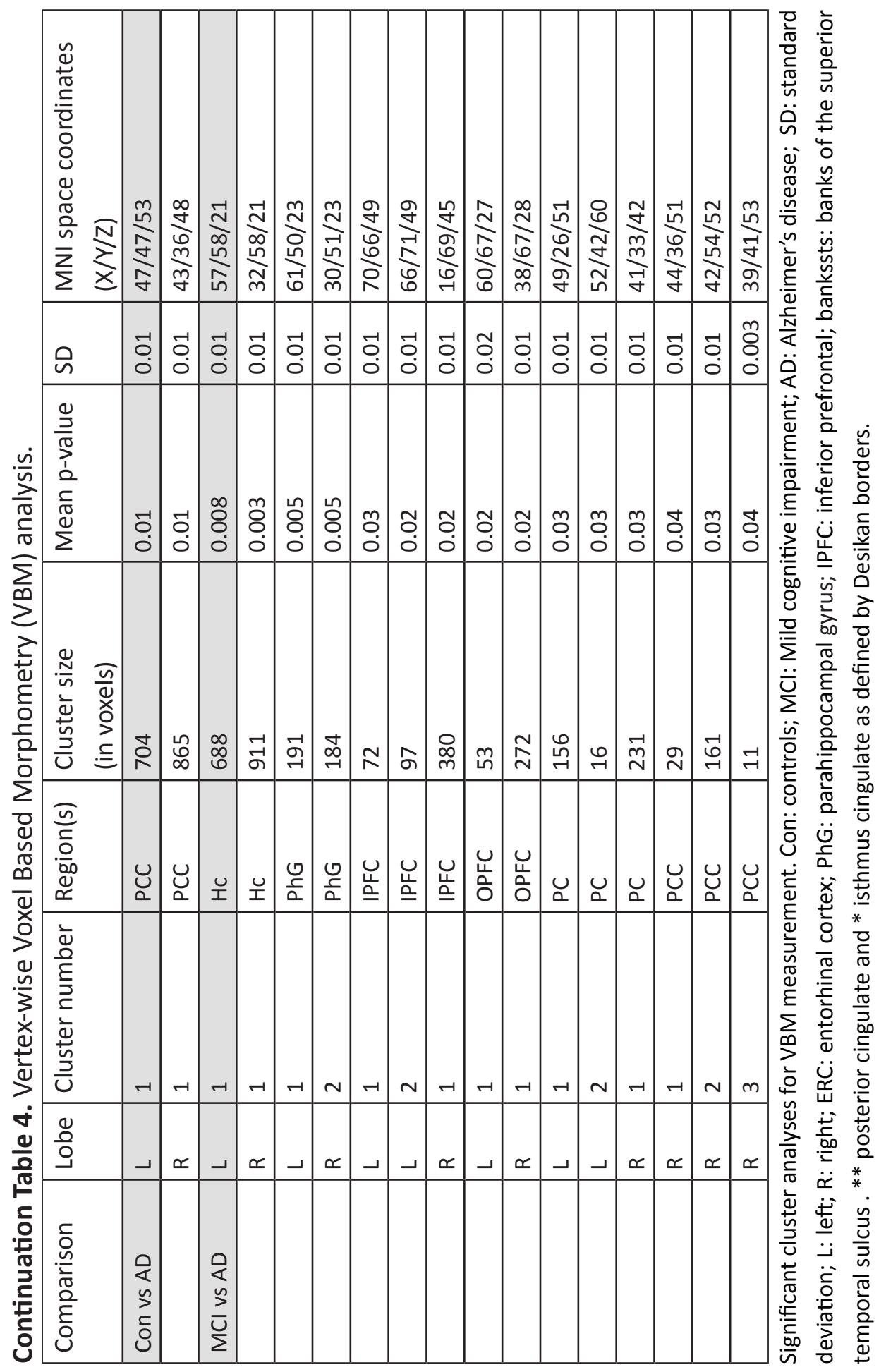




\section{DISCUSSION}

The present study showed that four different techniques used to assess gray matter atrophy in the brain show different results in the same dataset. When comparing controls with $\mathrm{MCl}$-patients, manual volumetric and VBM measurements show abnormalities in the MTL region whereas automated volumetric measurements show abnormalities in PPC but not in MTL regions. In AD, abnormalities in the PPC regions (manual volumetric measurements, $C T$ and $V B M$ ) and less pronounced in PFC regions (manual volumetric measurements, $C T$ and $V B M$ ), become apparent. Differences between early $(\mathrm{MCl})$ and late $(A D)$ phases of the disease were most noticeable in PFC regions (manual volumetric measurements and VBM).

This is to our knowledge the first study directly comparing four different measures to assess gray matter atrophy in the brain. Previous studies assessing gray matter atrophy patterns in AD mainly focused on MTL regions [51-55]. This study evaluates the discriminative power of each of these measurements, in and outside the MTL.

\section{Differences between techniques}

In the early phase of the disease when differentiation between controls and $\mathrm{MCl}$ is valuable, manual volumetric measurements and VBM were both able to detect differences in the MTL (PhG and Hc respectively) whereas automated FreeSurfer segmentation was sensitive to differences in the posterior parietal lobe. This is in line with current evidence stating that both manual volumetric measurements $[10,56,57]$ and VBM are sensitive in an early stage, with a predominance of MTL regions [58-60]. A recent study found that brain volume differences measured by VBM were already present at an early presymptomatic stage of $A D$, when subjects had no memory complaint or measurable cognitive impairment that would allow a clinical diagnosis of $\mathrm{MCl}$ or dementia according to current criteria [50]. It is furthermore known from the literature that automated segmentation techniques such as FreeSurfer are less suitable for hippocampal segmentation due to a frequent overestimation of hippocampal tissue volume leading to false-negative results [61]. This method seems however accurate in differentiating between controls and $\mathrm{MCl}$ in the parietal lobe $[62,63]$, which is again in line with our findings.

In later stages of the disease, manual and VBM measurements showed significant group differences in MTL, PPC and PFC regions. Automated segmentation 
and, less pronounced, cortical thickness measurements were sensitive to differences in MTL and PPC, but not in PFC regions.

For differential diagnosis and to monitor disease progression, it is also relevant to investigate which techniques were able to detect affected regions between subjects with $\mathrm{MCl}$ and $\mathrm{AD}$. Manual volumetry and VBM were able to differentiate $\mathrm{MCl}$ and $\mathrm{AD}$ patients in the $\mathrm{PhG}$ (manual volumetry), hippocampus (VBM), IPFC, OPFC and PC. Automated volumetry was able to differentiate $\mathrm{MCl}$ and $A D$ in the right $P C C$, and cluster based CT-analysis in the left precuneus.

\section{Regional abnormalities}

Our study showed that regional distribution of gray matter abnormalities was technique-dependent. Manual volumetric segmentation and VBM first showed abnormalities in the MTL regions, followed by PPC and PFC regions in the later stages. Automated volumetric measurements first showed abnormalities in the PPC region, followed by MTL regions. Abnormalities in cortical thickness became apparent later in the disease process, mainly in MTL and PPC regions. The increased cortical thickness found in the comparison between controls and AD was found in middlefrontal and orbitofrontal cortex areas, regions previously reported to show increased volume in older subjects [64]. The nature of this increase however remains unclear. In later stages of the disease only manual volumetric and VBM measurements were able to detect differences between controls and $A D$ in PFC regions.

\section{Atrophy progression in Alzheimer's disease}

Based on the current literature and our findings a sequence of brain abnormalities in $A D$ can be formulated. Gray matter atrophy initially starts in the anterior parahippocampal gyrus (entorhinal cortex region), then spreads to other medial temporal lobe (hippocampus) and posterior parietal regions (posterior cingulate and precuneus regions) $[50,65-67]$. In later stages of the disease, pathology extends to prefrontal cortex regions [68]. This pattern is consistent with the spread of $A D$ pathology evidenced by histopathology.

\section{Limitations}

The present study had several limitations. Since only cross-sectional data were available, no evaluation of predictive value for early Alzheimer's disease can be 
made for each of these techniques. Only males were included in this study in order to reduce interindividual variation. Generalisation of our findings to females should therefore be done with caution. Previous studies did however not reveal substantial genderrelated differences with respect to atrophy in AD. In this study, efforts were made towards an equality of anatomical boundaries for all of the investigated techniques. Although differences between ROI's were small, borders of manual, automated and VBM measurements were not entirely overlapping. This could have led to some bias in the dataset. Furthermore, all techniques assessed gray matter atrophy but approaches of each technique differed. For example, the comparison of VBM with manual and automated volumetric measurements is not straightforward since VBM analyzes the brain by a voxel-wise analysis compared to the ROI-approach in volumetric measurement. The same is true for the CT ROI-measurement where the mean thickness of gray matter in a ROI is analyzed compared to total cortical volume of a ROI in the volumetric approaches. However, the power of each technique in discriminating between groups was analyzed in a similar statistical manner (with a same amount of statistical corrections). Finally, in this study only FreeSurfer automated volumetry was evaluated. Other (atlasbased) automated measurements, which are outside the scope of this study, are also valuable for evaluation of gray matter atrophy in $\operatorname{AD}[12,69,70]$.

\section{Clinical implications}

Manual volumetry can still be considered as the gold standard for the evaluation

of gray matter atrophy in $\operatorname{AD}[10,56,57]$. The discriminative power of manual volumetric measurements is however restricted to the MTL in the early phase. Since medial temporal lobe regions such as the parahippocampal gyrus are among the first areas affected by the disease [7], measurement of its volume is clinically important and manual volumetric measurements, however time-consuming, could complement standard clinical practice by reporting GM abnormalities at the single subject-level. Despite the methodological considerations discussed in the introduction, VBM is also sensitive in the early stage. VBM is however less suitable for clinical implementation due to it's incapability for reporting reduced GM volume at the single subject-level. A recent study [71] showed that the predictive accuracy for AD-conversion in subjects with $\mathrm{MCl}$ is similar for automated (LEAP segmentation) [70] and manual hippocampal measurement. However, the automated measurement used in our study (FreeSurfer), seems less suitable for 
evaluation of medial temporal lobe atrophy in the early phase, but can complement the diagnostic process by assessing the degree of (posterior) parietal atrophy [72]. Evaluation of both medial temporal and posterior parietal regions in the early phase can strengthen the diagnosis of underlying AD pathology.

\section{CONCLUSION}

We conclude that the discriminative ability of different techniques measuring gray matter atrophy in the brain is region-specific. Manual volumetric measurements together with VBM are preferred showing abnormalities in most of the investigated regions, with a predominance of the MTL in the early phase. Automated volumetric measurements show similar performances in the early phase of the disease, displaying significant differences in the PPC but show limited discriminative power in MTL regions. Automated measurements of cortical thickness are less sensitive in the $\mathrm{MCl}$ stage and it's sensitivity is furthermore restricted to the MTL and PPC regions in later stages of the disease (AD). Our data furthermore suggests, in line with the literature, that patients suffering from $\mathrm{MCl}$ first show abnormalities in the MTL (PhG and hippocampus) and PPC (posterior cingulate and precuneus) followed by abnormalities in the PFC (orbitofrontal en inferior prefrontal cortex) in the later stages. 


\section{REFERENCES}

1. Petersen RC, Smith GE, Waring SC, Ivnik RJ, Tangalos EG, Kokmen E. Mild Cognitive Impairment: Clinical Characterization and Outcome. Archives of Neurology. 1999;56(3):303-8.

2. Eckerström C, Andreasson U, Olsson E, Rolstad S, Blennow K, Zetterberg H, et al. Combination of Hippocampal Volume and Cerebrospinal Fluid Biomarkers Improves Predictive Value in Mild Cognitive Impairment. Dementia and Geriatric Cognitive Disorders. 2010;29(4):294-300.

3. Galluzzi S, Geroldi C, Ghidoni R, Paghera B, Amicucci G, Bonetti M, et al. The new Alzheimer's criteria in a naturalistic series of patients with mild cognitive impairment. Journal of Neurology. 2010;257(12):2004-14.

4. Vemuri P, Wiste HJ, Weigand SD, Shaw LM, Trojanowski JQ, Weiner MW, et al. MRI and CSF biomarkers in normal, $\mathrm{MCl}$, and AD subjects. Neurology. 2009;73:294-301.

5. Chetelat G, Baron J-C. Early diagnosis of alzheimer's disease: contribution of structural neuroimaging. Neurolmage. 2003;18(2):525-41.

6. Zakzanis KK, Graham SJ, Campbell Z. A Meta-Analysis of Structural and Functional Brain Imaging in Dementia of the Alzheimer's Type: A Neuroimaging Profile. Neuropsychology Review. 2003;13(1):1-18.

7. Echávarri $C$, Aalten $P$, Uylings $H B M$, Jacobs $H$, Visser $P$, Gronenschild $E$, et al. Atrophy in the parahippocampal gyrus as an early biomarker of Alzheimer's disease. Brain Structure and Function. 2011;215(3):265-71.

8. Lerch JP, Pruessner J, Zijdenbos AP, Collins DL, Teipel SJ, Hampel H, et al. Automated cortical thickness measurements from MRI can accurately separate Alzheimer's patients from normal elderly controls. Neurobiology of Aging. 2008;29(1):23-30.

9. Weniger G, Irle E. Posterior parahippocampal gyrus lesions in the human impair egocentric learning in a virtual environment. European Journal of Neuroscience. 2006;24(8):2406-14.

10. Barnes J, Ourselin S, Fox NC. Clinical application of measurement of hippocampal atrophy in degenerative dementias. Hippocampus. 2009;19(6):510-6.

11. van de Pol LA, Barnes J, Scahill RI, Frost C, Lewis EB, Boyes RG, et al. Improved reliability of hippocampal atrophy rate measurement in mild cognitive impairment using fluid registration. Neurolmage. 2007;34(3):1036-41. 
12. Tisserand DJ, Pruessner JC, Sanz Arigita EJ, van Boxtel MPJ, Evans AC, Jolles J, et al. Regional Frontal Cortical Volumes Decrease Differentially in Aging: An MRI Study to Compare Volumetric Approaches and Voxel-Based Morphometry. Neurolmage. 2002;17(2):657-69.

13. Sanchez-Benavides G, Gomez-Anson B, Molinuevo JL, Blesa R, Monte GC, Buschke $\mathrm{H}$, et al. Medial Temporal Lobe Correlates of Memory Screening Measures in Normal Aging, $\mathrm{MCl}$, and AD. Journal of Geriatric Psychiatry and Neurology. 2010;23(2):100-8.

14. Im K, Lee J-M, Seo SW, Yoon U, Kim ST, Kim Y-H, et al. Variations in cortical thickness with dementia severity in Alzheimer's disease. Neuroscience Letters. 2008;436(2):227-31.

15. Julkunen V, Niskanen E, Koikkalainen J, Herukka S-K, Pihlajamäki M, Hallikainen $M$, et al. Differences in Cortical Thickness in Healthy Controls, Subjects with Mild Cognitive Impairment, and Alzheimer's Disease Patients: A Longitudinal Study. Journal of Alzheimer's Disease. 2010;21(4):1141-51.

16. Jacobs HIL, Van Boxtel MPJ, Gronenschild EHBM, Uylings HBM, Jolles J, Verhey FRJ. Decreased gray matter diffusivity: a potential early Alzheimer disease biomarker? . Alzheimer's \& Dementia. 2012.

17. Dickerson BC, Bakkour A, Salat DH, Feczko E, Pacheco J, Greve DN, et al. The Cortical Signature of Alzheimer's Disease: Regionally Specific Cortical Thinning Relates to Symptom Severity in Very Mild to Mild AD Dementia and is Detectable in Asymptomatic Amyloid-Positive Individuals. Cerebral Cortex. 2009;19(3):497-510.

18. Ashburner J, Friston KJ. Voxel-Based Morphometry, The Methods. Neurolmage. 2000;11(6):805-21.

19. Good CD, Johnsrude IS, Ashburner J, Henson RNA, Friston KJ, Frackowiak RSJ. A Voxel-Based Morphometric Study of Ageing in 465 Normal Adult Human Brains. Neurolmage. 2001;14(1):21-36.

20. Barnes J, Scahill RI, Boyes RG, Frost C, Lewis EB, Rossor CL, et al. Differentiating $A D$ from aging using semiautomated measurement of hippocampal atrophy rates. Neurolmage. 2004;23(2):574-81.

21. Kennedy KM, Erickson KI, Rodrigue KM, Voss MW, Colcombe SJ, Kramer AF, et al. Age-related differences in regional brain volumes: A comparison of optimized voxel-based morphometry to manual volumetry. Neurobiology of Aging. 2009;30(10):1657-76.

112 | Chapter 4: Sensitivity of different MRI-techniques 
22. Bookstein FL. Voxel-Based Morphometry Should Not Be Used with Imperfectly Registered Images. Neurolmage. 2001;14(6):1454-62.

23. Burgmans $S$, van Boxtel MPJ, Smeets F, Vuurman EFPM, Gronenschild EHBM, Verhey FRJ, et al. Prefrontal cortex atrophy predicts dementia over a six-year period. Neurobiology of Aging. 2009;30(9):1413-9.

24. Jacobs HIL, Van Boxtel MPJ, Uylings HBM, Gronenschild EHBM, Verhey FR, Jolles J. Atrophy of the parietal lobe in preclinical dementia. Brain and Cognition. 2011;75(2):154-63.

25. Van der Elst W, van Boxtel MP, van Breukelen GJ, Jolles J. Rey's verbal learning test: normative data for 1855 healthy participants aged 24-81 years and the influence of age, sex, education, and mode of presentation. J Int Neuropsychol Soc. 2005 May;11(3):290-302.

26. Folstein MF, Folstein SE, McHugh PR. "Mini-mental state". A practical method for grading the cognitive state of patients for the clinician. J Psychiatr Res. 1975 Nov;12(3):189-98.

27. Petersen RC, Smith GE, Waring SC, Ivnik RJ, Tangalos EG, Kokmen E. Mild cognitive impairment: clinical characterization and outcome. Arch Neurol. 1999 Mar;56(3):303-8.

28. Petersen RC, Doody R, Kurz A, Mohs RC, Morris JC, Rabins PV, et al. Current concepts in mild cognitive impairment. Arch Neurol. 2001 Dec;58(12):1985-92.

29. APA APA. Diagnostic and Statistical Manual of Mental Disorders, 4th edition. Washington, D.C.1994.

30. McKhann G, Drachman D, Folstein M, Katzman R, Price D, Stadlan EM. Clinical diagnosis of Alzheimer's disease: report of the NINCDS-ADRDA Work Group under the auspices of Department of Health and Human Services Task Force on Alzheimer's Disease. Neurology. 1984 Jul;34(7):939-44.

31. Nylenna M, Riis P. Identification of patients in medical publications: need for informed consent. British Medical Journal. 1991;302(6789):1182.

32. Gronenschild EH, Burgmans S, Smeets F, Vuurman EF, Uylings HB, Jolles J. A time-saving and facilitating approach for segmentation of anatomically defined cortical regions: MRI volumetry. Psychiatry Res. 2010 Mar 30;181(3):211-8.

33. Burgmans $\mathrm{S}$, van Boxtel MPJ, van den Berg KEM, Gronenschild EHBM, Jacobs HIL, Jolles J, et al. The posterior parahippocampal gyrus is preferentially affected in age-related memory decline. Neurobiology of Aging. 2011;32(9):1572-8.

34. Jones BF, Barnes J, Uylings HBM, Fox NC, Frost C, Witter MP, et al. Differential 
Regional Atrophy of the Cingulate Gyrus in Alzheimer Disease: A Volumetric MRI Study. Cerebral Cortex. 2006;16(12):1701-8.

35. Ryu SY, Kwon MJ, Lee SB, Yang DW, Kim TW, Song IU, et al. Measurement of precuneal and hippocampal volumes using magnetic resonance volumetry in Alzheimer's disease. Journal of clinical neurology (Seoul, Korea). 2010;6(4):196203.

36. Shrout PE, Fleiss JL. Intraclass correlations: uses in assessing rater reliability. Psychol Bull. 1979 Mar;86(2):420-8.

37. Smith SM. Fast robust automated brain extraction. Human Brain Mapping. 2002;17(3):143-55.

38. Benjamini Y, Hochberg Y. Controlling the False Discovery Rate: A Practical and Powerful Approach to Multiple Testing. Journal of the Royal Statistical Society Series B (Methodological) 1995;57(1):289-300.

39. Gronenschild EHBM, Habets P, Jacobs HIL, Mengelers R, Rozendaal N, van Os J, et al. The Effects of FreeSurfer Version, Workstation Type, and Macintosh Operating System Version on Anatomical Volume and Cortical Thickness Measurements. PLoS ONE. 2012;7(6):e38234.

40. Fischl B, Salat DH, Busa E, Albert M, Dieterich M, Haselgrove C, et al. Whole Brain Segmentation: Automated Labeling of Neuroanatomical Structures in the Human Brain. Neuron. 2002;33(3):341-55.

41. Fischl B, Dale AM. Measuring the Thickness of the Human Cerebral Cortex from Magnetic Resonance Images,. Proceedings of the National Academy of Sciences. 2000;97:11044-9.

42. Desikan RS, Ségonne F, Fischl B, Quinn BT, Dickerson BC, Blacker D, et al. An automated labeling system for subdividing the human cerebral cortex on MRI scans into gyral based regions of interest. Neurolmage. 2006;31(3):968-80.

43. Smith SM, Jenkinson M, Woolrich MW, Beckmann CF, Behrens TEJ, JohansenBerg $\mathrm{H}$, et al. Advances in functional and structural MR image analysis and implementation as FSL. Neurolmage. 2004;23, Supplement 1(0):S208-S19.

44. Zhang Y, Brady M, Smith S, . Segmentation of brain MR images through a hidden Markov random field model and the expectation maximization algorithm. . IEEE Trans on Medical Imaging. 2001;20(1):45-57.

45. Jenkinson M, Bannister PR, Brady JM, Smith S. Improved optimisation for the robust and accurate linear registration and motion correction of brain images. . Neurolmage. 2002;17(2):825-41. 
46. Jenkinson $M$, Smith S. A global optimisation method for robust affine registration of brain images. . Medical Image Analysis. 2001;5(2):143-56.

47. Andersson JLR, Jenkinson M, Smith S. Non-linear optimisation. 2007a.

48. Andersson JLR, Jenkinson M, Smith S. Non-linear registration, aka Spatial normalisation 2007b.

49. Rueckert D, Sonoda LI, Hayes C, Hill DLG, Leach MO, Hawkes DJ. Non-rigid registration using free-form deformations: Application to breast MR images. . IEEE Transactions on Medical Imaging. 1999;18(8):712-21.

50. Tondelli M, Wilcock GK, Nichelli P, De Jager CA, Jenkinson M, Zamboni G. Structural MRI changes detectable up to ten years before clinical Alzheimer's disease. Neurobiology of Aging. 2012;33(4):825.e25-.e36.

51. Kalus P, Slotboom J, Gallinat Jr, Mahlberg R, Cattapan-Ludewig K, Wiest R, et al. Examining the gateway to the limbic system with diffusion tensor imaging: The perforant pathway in dementia. Neurolmage. 2006;30(3):713-20.

52. Lehmann M, Douiri A, Kim LG, Modat M, Chan D, Ourselin S, et al. Atrophy patterns in Alzheimer's disease and semantic dementia: A comparison of FreeSurfer and manual volumetric measurements. Neurolmage. 2010;49(3):2264-74.

53. Pantel J, Häger DR, Kratz B, Minnemann E, Martin M, Schad LR, et al. Strukturelle zerebraleVeranderungen beiProbanden mitleichterkognitiver Beeintrachtigung Eine MR-volumetrische Studie. Der Nervenarzt. 2002;73(9):845-50.

54. Rogalski EJ, Murphy CM, deToledo-Morrell L, Shah RC, Moseley ME, Bammer $\mathrm{R}$, et al. Changes in parahippocampal white matter integrity in amnestic mild cognitive impairment: A diffusion tensor imaging study. Behavioural Neurology. 2009;21(1):51-61.

55. Teipel S, Pruessner J, Faltraco F, Born C, Rocha-Unold M, Evans A, et al. Comprehensive dissection of the medial temporal lobe in $A D$ : measurement of hippocampus, amygdala, entorhinal, perirhinal and parahippocampal cortices using MRI. Journal of Neurology. 2006;253(6):794-800.

56. Boccardi M, Ganzola R, Bocchetta M, Pievani M, Redolfi A, Bartzokis G, et al. Survey of Protocols for the Manual Segmentation of the Hippocampus: Preparatory Steps Towards a Joint EADC-ADNI Harmonized Protocol. Journal of Alzheimer's Disease. 2011;26:61-75.

57. van de Pol LA, van der Flier WM, Korf ESC, Fox NC, Barkhof F, Scheltens $P$. Baseline predictors of rates of hippocampal atrophy in mild cognitive 
impairment. Neurology. 2007;69(15):1491-7.

58. Frisoni GB, Testa C, Zorzan A, Sabattoli F, Beltramello A, Soininen $H$, et al. Detection of grey matter loss in mild Alzheimer's disease with voxel based morphometry. Journal of Neurology, Neurosurgery \& Psychiatry. 2002;73(6):657-64.

59. Chetelat G, Landeau B, Eustache F, Mezenge F, Viader F, de la Sayette V, et al. Using voxel-based morphometry to map the structural changes associated with rapid conversion in $\mathrm{MCl}$ : A longitudinal MRI study. Neurolmage. 2005;27(4):934-46.

60. Whitwell JL, Przybelski SA, Weigand SD, Knopman DS, Boeve BF, Petersen RC, et al. 3D maps from multiple MRI illustrate changing atrophy patterns as subjects progress from mild cognitive impairment to Alzheimers disease. . Brain: A journal of neurology. 2007;130(7):1777-86.

61. Sanchez-Benavides G, Gomez-Ansun B, Sainz A, Vives Y, Delfino M, PeoaCasanova J. Manual validation of FreeSurfer's automated hippocampal segmentation in normal aging, mild cognitive impairment, and Alzheimer Disease subjects. Psychiatry Research: Neuroimaging. 2010;181(3):219-25.

62. Li C, Wang J, Li G, Zheng J, Liu C, Du H. Alterations of Whole-Brain Cortical Area and Thickness in Mild Cognitive Impairment and Alzheimer's Disease. Journal of Alzheimer's Disease. 2011;27(2):281-90.

63. GreeneSJ, Killiany RJ. Subregions of the inferior parietal lobule are affected in the progression to Alzheimer's disease. Neurobiology of Aging. 2010;31(8):130411.

64. Salat DH, Kaye JA, Janowsky JS. Greater Orbital Prefrontal Volume Selectively Predicts Worse Working Memory Performance in Older Adults. Cerebral Cortex. 2002;12(5):494-505.

65. Busatto GF, Diniz BS, Zanetti MV. Voxel-based morphometry in Alzheimer's disease. Expert Review of Neurotherapeutics. 2008 2012/08/05;8(11):1691702.

66. Karas GB, Scheltens P, Rombouts SARB, Visser PJ, van Schijndel RA, Fox NC, et al. Global and local gray matter loss in mild cognitive impairment and Alzheimer's disease. Neurolmage. 2004;23(2):708-16.

67. Jacobs HIL, Van Boxtel MPJ, Jolles J, Verhey FRJ, Uylings HBM. Parietal cortex matters in Alzheimer's disease: An overview of structural, functional and metabolic findings. Neuroscience \& Biobehavioral Reviews. 2011(0). 
68. Scahill RI, Schott JM, Stevens JM, Rossor MN, Fox NC. Mapping the evolution of regional atrophy in Alzheimer's disease: Unbiased analysis of fluid-registered serial MRI. Proceedings of the National Academy of Sciences. 2002;99(7):47037.

69. Tabert M, Manly JJ, Liu X. Neuropsychological prediction of conversion to alzheimer disease in patients with mild cognitive impairment. Archives of General Psychiatry. 2006;63(8):916-24.

70. Wolz R, Aljabar P, Hajnal JV, Hammers A, Rueckert D. LEAP: Learning embeddings for atlas propagation. Neurolmage. 2010;49(2):1316-25.

71. Clerx L, van Rossum I, Burns L, Knol DL, Scheltens P, Verhey FRJ, et al. Comparison of measurements of medial temporal lobe atrophy in the prediction of Alzheimer's Disease in subjects with MCl. Alzheimer's \& dementia : the journal of the Alzheimer's Association. 2011;7(4):S133.

72. Jacobs HIL, Van Boxtel MPJ, van der Elst W, Burgmans S, Smeets F, Gronenschild EHBM, et al. Increasing the Diagnostic Accuracy of Medial Temporal Lobe Atrophy in Alzheimer's Disease. Journal of Alzheimer's Disease. 2011;25(3):47790.

73. Filipek PA, Richelme C, Kennedy DN, Caviness VS. The Young Adult Human Brain: An MRI-based Morphometric Analysis. Cerebral Cortex. 1994;4(4):34460.

74. Uylings HBM, Arigita EJS, de Vos K, Pool CW, Evers P, Rajkowska G. 3-D Cytoarchitectonic parcellation of human orbitofrontal cortex: Correlation with postmortem MRI. Psychiatry Research: Neuroimaging. 2010;183(1):1-20. 
SUPPLEMENTAL MATERIAL

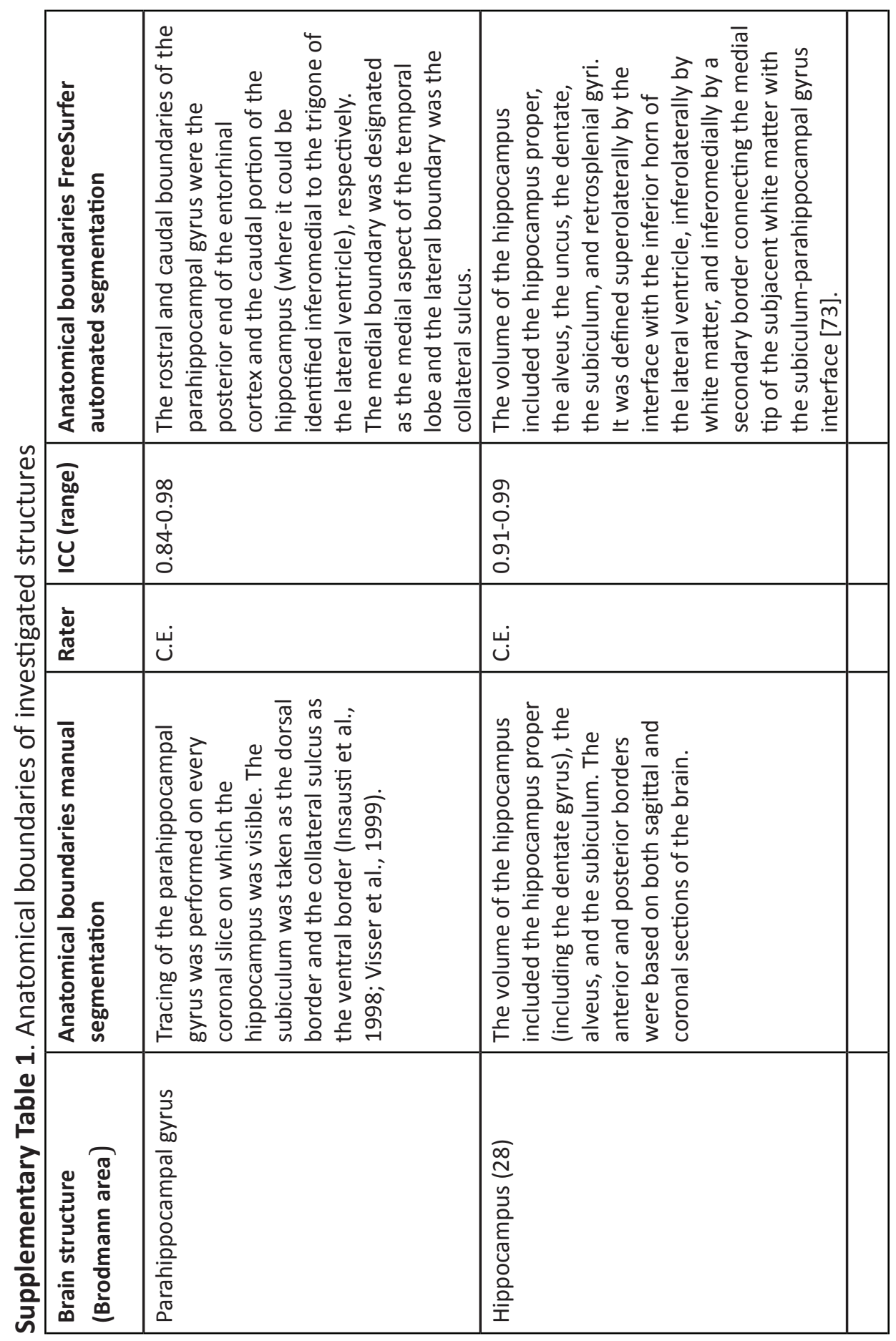

118 | Chapter 4: Sensitivity of different MRI-techniques 


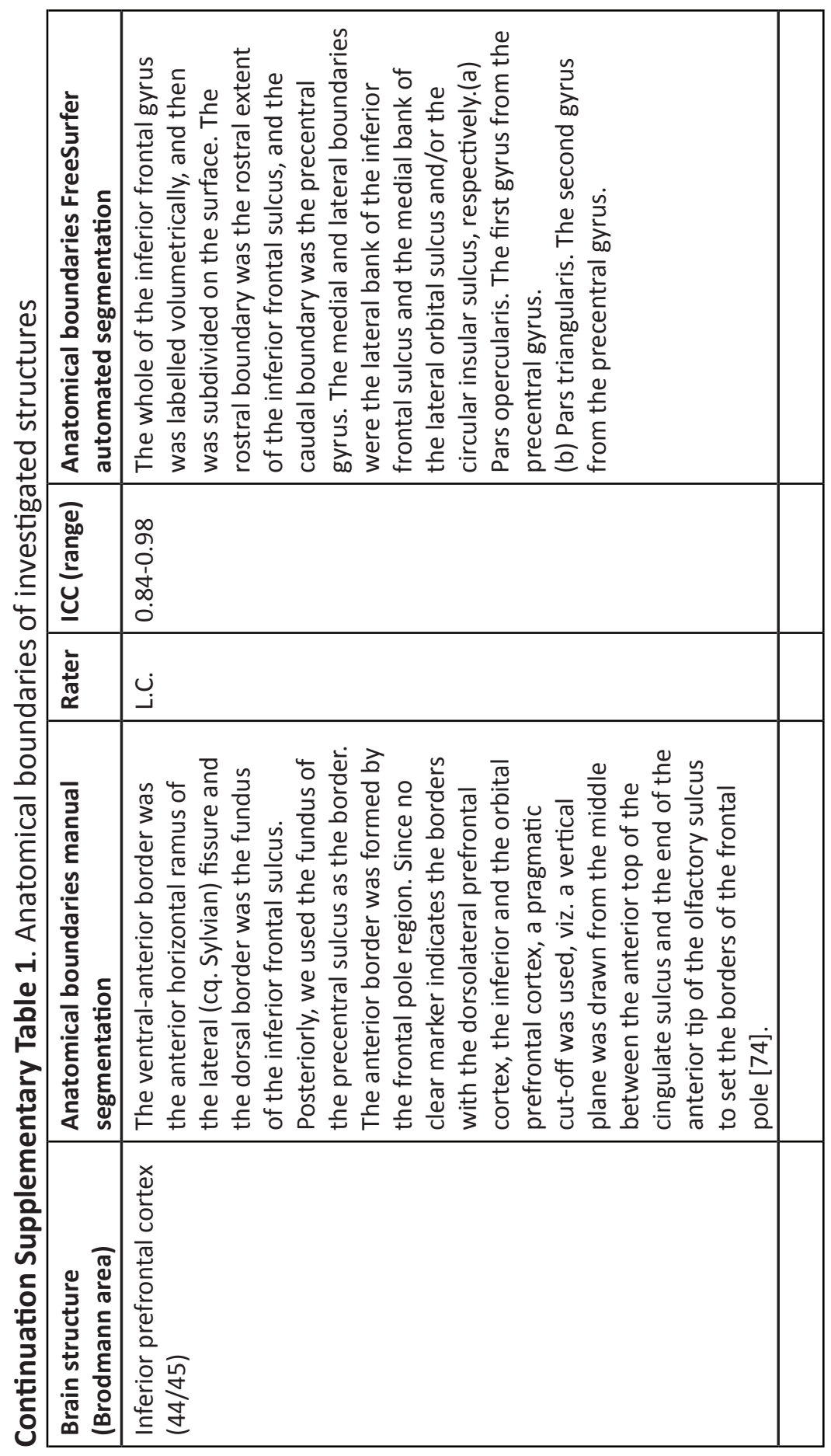




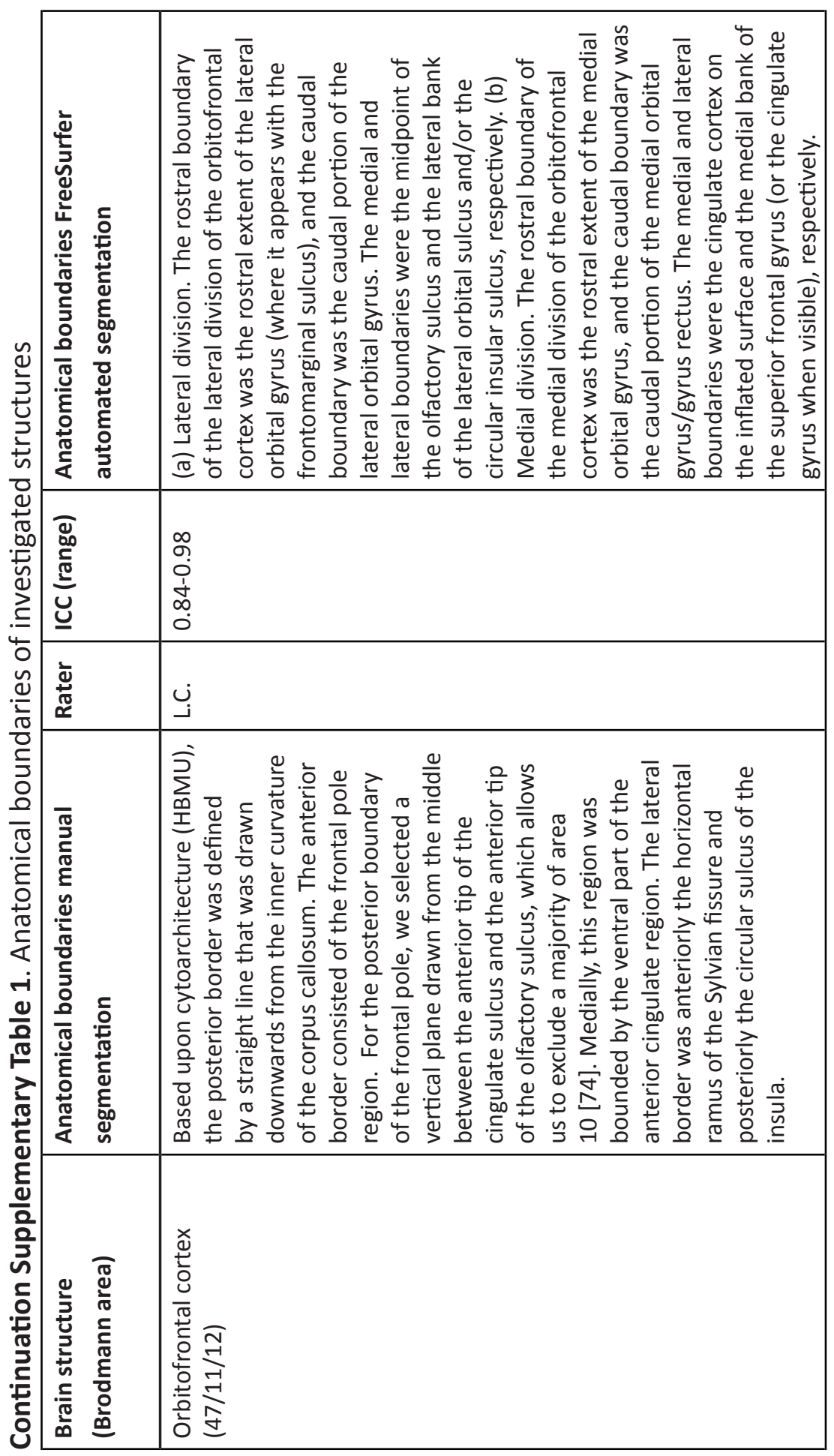




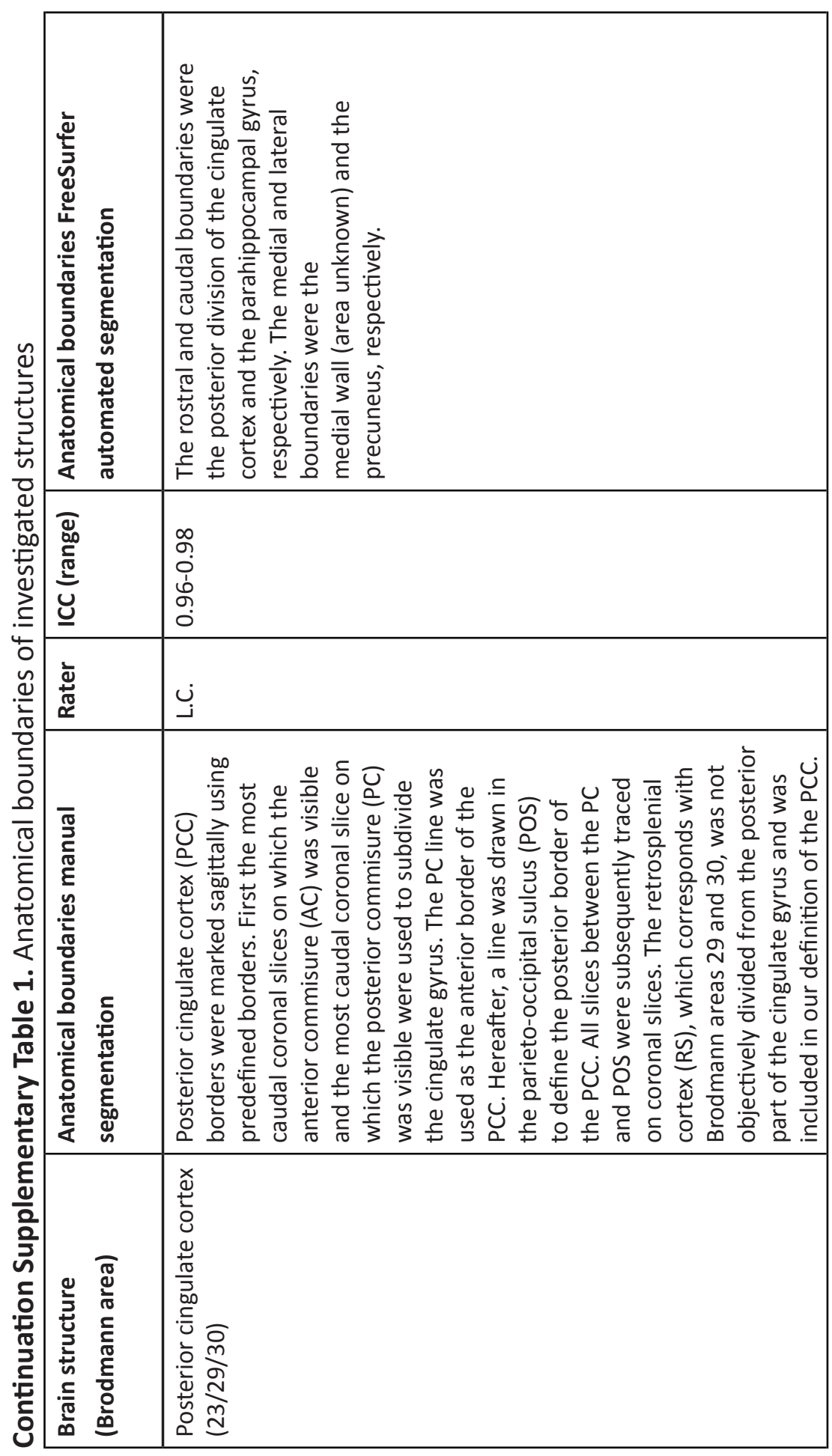




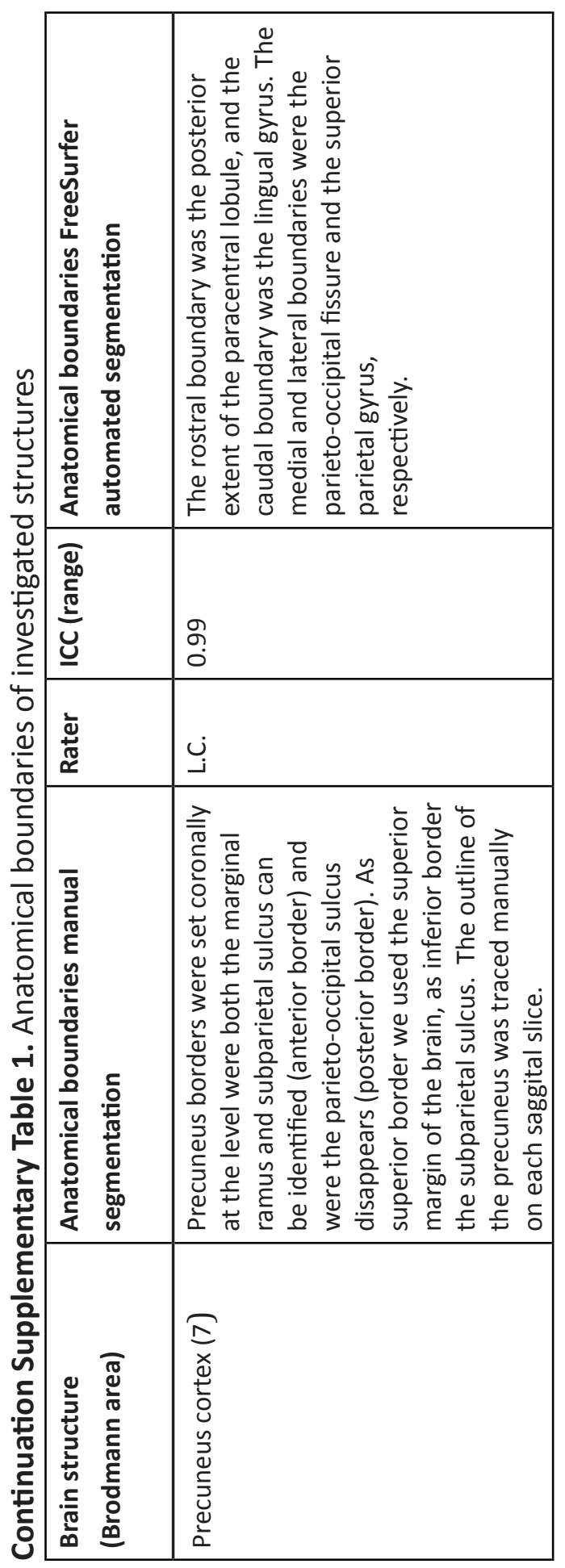

122 | Chapter 4: Sensitivity of different MRI-techniques 


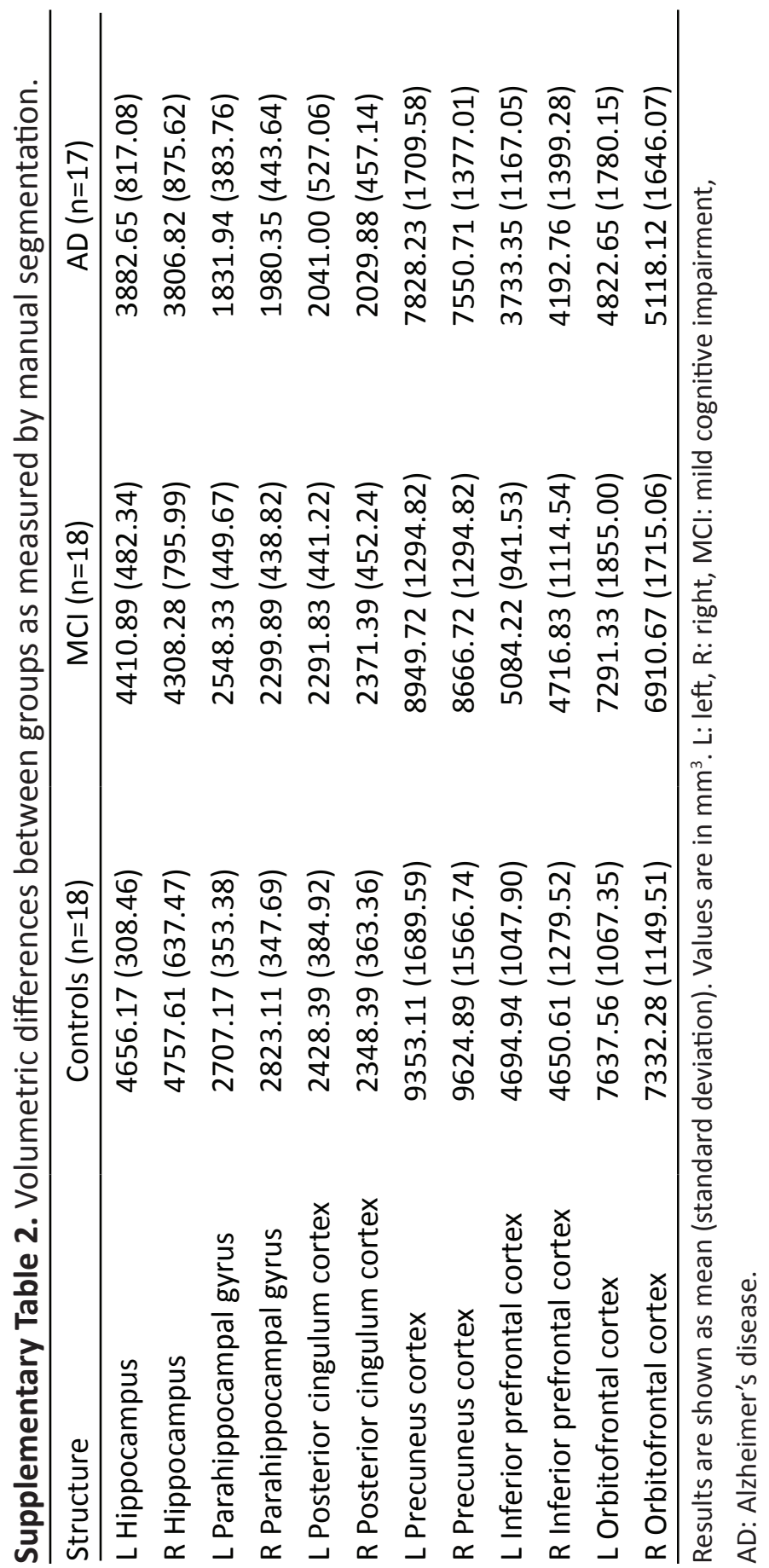

Chapter 4: Sensitivity of different MRI-techniques | 123 


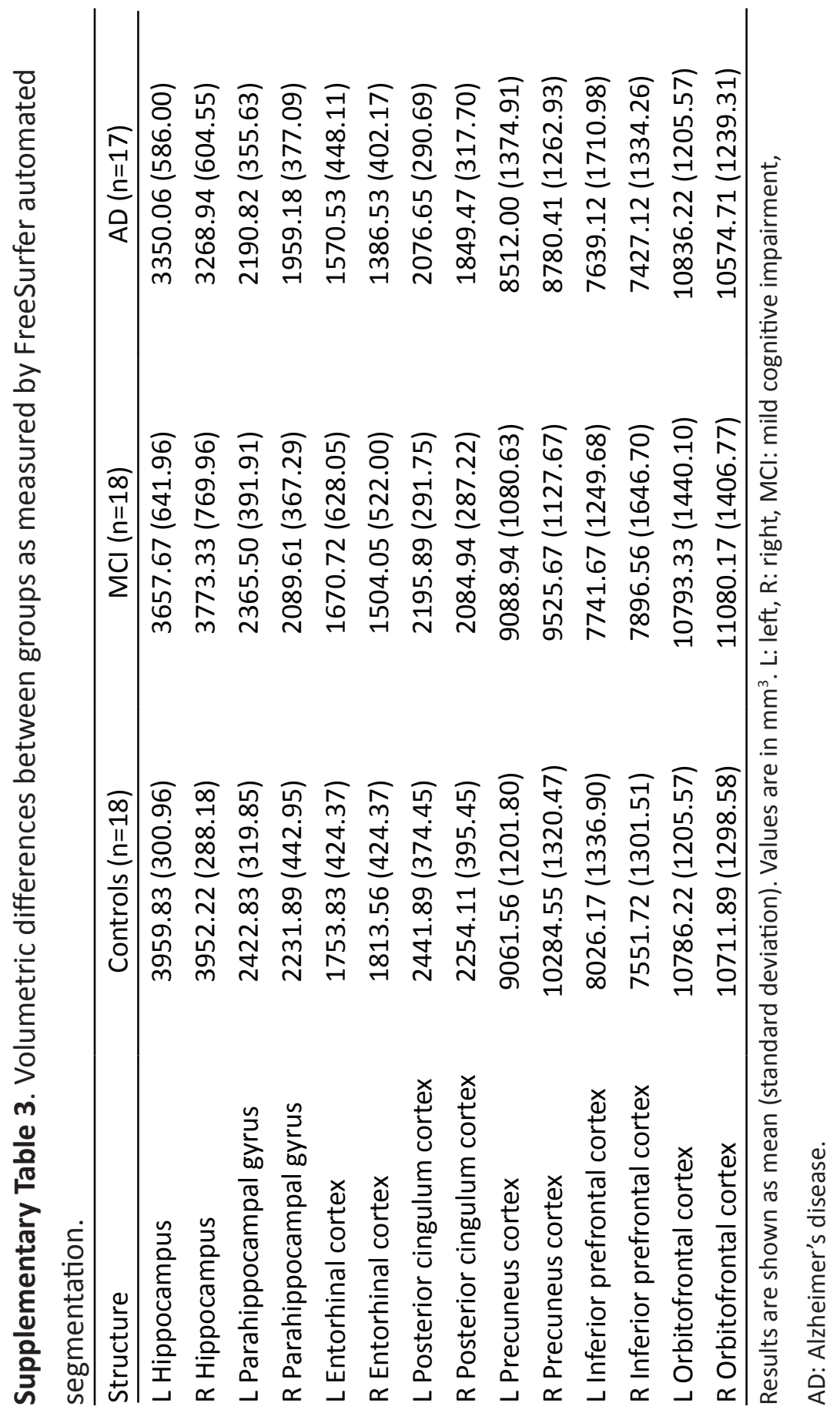





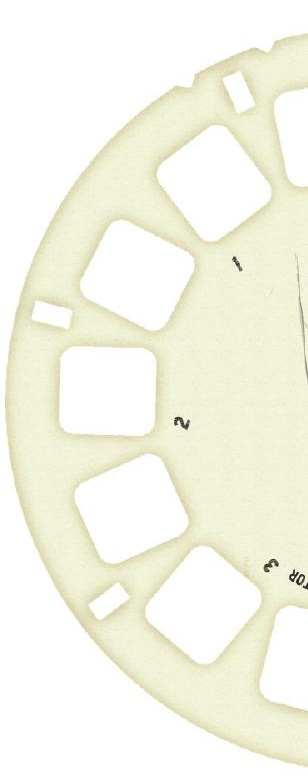




\section{Chapter 5}

New MRI markers for AD: a metaanalysis of diffusion tensor imaging and a comparison with medial temporal lobe measurements

Journal of Alzheimer's Disease. 2012;29(2):405-29

$\uparrow$

c) 


\section{ABSTRACT}

The aim of the present study is to evaluate the diagnostic value of diffusion tensor imaging (DTI) for early Alzheimer's disease (AD) in comparison to widely accepted medial temporal lobe (MTL) atrophy measurements. A systematic literature research was performed into DTI and MTL atrophy in AD and mild cognitive impairment (MCI). We included seventy-six studies on MTL atrophy including 8122 subjects and fifty-five DTI studies including 2791 subjects. Outcome measure was the effect size (ES) expressed as Hedges g. In volumetric studies, atrophy of the MTL significantly differentiated between $A D$ and controls (ES 1.32-1.98) and $\mathrm{MCl}$ and controls (ES 0.61-1.46). In DTI-Fractional anisotropy (FA) studies, the total cingulum differentiated best between $A D$ and controls $(E S=1.73)$ and the parahippocampal cingulum between $\mathrm{MCl}$ and controls ( $E S=0.97)$. In DTI-Mean diffusivity (MD) studies, the hippocampus differentiated best between $A D$ and controls ( $E S=-1.17$ ) and between $\mathrm{MCl}$ and controls (ES=-1.00). We can conclude that in general, the ES of volumetric MTL atrophy measurements was equal or larger than that of DTI measurements. However, for the comparison between controls and $\mathrm{MCl}$-patients, ES of hippocampal MD was larger than ES of hippocampal volume. Furthermore, it seems that MD values have somewhat more discriminative power than FA values with higher ES in the frontal, parietal, occipital and temporal lobe. 


\section{INTRODUCTION}

Alzheimer's disease (AD), the most common cause of dementia, affects about 33.9 million people worldwide. Over the next forty years, the prevalence of $A D$ is expected to triple due to the aging population [1]. This creates an urgent need for early diagnosis of the disease to enable treatment at an early stage. Many ADrelated research has focused on mild cognitive impairment $(\mathrm{MCl})$, a transitional state between normal aging and early $A D[2,3]$. Estimates of annual conversion from $\mathrm{MCl}$ to $A D$ show a great variability, from $5 \%$ to $40 \%$ per year [4], depending primarily upon the setting in which the studies were carried out (clinical versus populationbased studies).Medial temporal lobe (MTL) atrophy as assessed on structural magnetic resonance imaging (MRI) has proven to be an effective clinical aid in the early diagnosis of $A D$ [5], and this method could predict $A D$ in subjects with $\mathrm{MCl}$ [68]. Still, the diagnostic accuracy of MTL atrophy for AD is only moderately high [9].

Recently, MRI techniques have become available which can measure the integrity of cerebral white matter (WM). It has been shown that WM integrity is decreased in subjects with $\mathrm{MCl}$ and $\mathrm{AD}$ suggesting a potential diagnostic value that may possibly help to improve the diagnostic accuracy of MTL atrophy measurements $[10,11]$. Integrity of WM tracts can be assessed using diffusion tensor imaging (DTI), which measures the diffusion of water molecules in neural tissue. By means of DTI one can detect whole-brain diffusional abnormalities at a stage where macrostructural changes may not have become visible yet. Hence, DTI may be an early marker for neurodegeneration. DTI-studies mostly use fractional anisotropy (FA) and mean diffusivity (MD) measurements in a priori defined regions of interest (ROI) or whole-brain voxel wise analyses to evaluate the integrity of WM in subjects with $\mathrm{AD}$ and $\mathrm{MCl}$. FA is a measure of anisotropic water diffusion and reflects the degree of directional diffusion in cellular structures within the fiber tracts. By using FA one can evaluate fiber density, axonal diameter and myelination in WM [12]. A decrease in FA indicates a loss of fiber tract integrity and thus WM damage [13]. MD is a measure of diffusion in the noncolinear direction of free diffusion (translational diffusion). MD reflects the average diffusion within a voxel [13, 14]. An increase in $M D$ represents a loss of anisotropy due to an increase in free water diffusion [15]. Abnormally decreased $F A$ and increased $\mathrm{MD}$ in subjects with $\mathrm{MCl}$ and $\mathrm{AD}$ compared to controls suggests tissue damage which is consistent with neuropathological data 
demonstrating partial loss of myelin, axons, and oligodendrial cells in white matter [16].

To date there have been several descriptive reviews of DTI studies of $A D$ and $M C l$ $[15,17,18]$ and one review recently quantified the diagnostic value of DTI in subjects with $\mathrm{MCl}$ and $A D$ [19]. The aim of the present study was to evaluate the potential diagnostic value of DTI for early AD in comparison to widely accepted MTL atrophy measurements by using a meta-analysis approach. We tested the ability of MTL and DTI measurements to discriminate between controls, subjects with $\mathrm{MCl}$, and subjects with AD-type dementia. This meta-analysis is the first to quantify the potential diagnostic value of DTI in comparison with widely used MTL atrophy measurements. Furthermore, it is the first time that effect size (ES) measures between subjects with $\mathrm{MCl}$ and $\mathrm{AD}$ are compared with each other. This is important from a clinical point of view, as it indicates the degree of overlap between $\mathrm{MCl}$ and AD.

\section{METHOD}

\section{Search strategy}

An online literature search of the database PUBMED was conducted by one rater (LC) up to August 2011. The keywords used for searching volumetric MTL and DTI studies are listed in Table 1. Abstracts of the retrieved articles were screened for relevance. Searching the references of relevant papers identified further articles for inclusion.

\section{Data selection}

Inclusion criteria were: (i) the study included subjects with AD and/or $\mathrm{MCl}$; (ii) the data on average DTI and/or MTL values and standard deviation were provided; (iii) the article was limited to humans; and (iv) the article was written in English, Dutch, German, or French. If raw scores were not presented in the article, authors were contacted and resulting data were added to the meta-analysis. Since ES was calculated with correction for sample size (see below), studies with a relatively small sample size in one of the groups $(n<10)$ were also included. We excluded MTL-studies that did not correct for intracranial volume (ICV), post mortem studies and studies using patient subgroups of 'vascular' MCl or AD. For 
the DTI articles, limitation of the search to the time period of the last 10 years ensured that the scanning techniques met current standards. We included DTI studies that used different methods of extracting DTI data: manual segmentation, (semi-) automated ROI or tractography. Studies using whole-brain voxelwise analyses (VBM) and tract-based spatial statistics (TBSS) were also included.

\section{Data extraction and analysis}

One rater (LC) recorded the mean and standard deviation of all the investigated structures for each subgroup (controls, $\mathrm{MCl}, \mathrm{AD}$ ) by means of a data extraction form using Microsoft Excel (Microsoft Corporation, Redmond, USA). Data on different MTL structures (hippocampus, entorhinal cortex and parahippocampal gyrus) were extracted separately. Values in ROI were taken from the average of left and right hemisphere. Articles that reported the visually rated MTA score [20] were also included. Diffusional data were classified in the same way, according to region and DTI modality. DTI- measurements of the following regions were included in the meta-analysis: frontal, parietal, temporal, or occipital lobe white matter (WM), hippocampus, parahippocampal gyrus, anterior, middle, posterior, parahippocampal and total cingulum, anterior and posterior corpus callosum (CC), uncinate fasciculus (UF), superior longitudinal fasciculus (SLF), and internal capsule. To correct for small sample size, ES was calculated using Hedge's g, which is the difference between group means divided by the pooled standard deviation [21]. Effect sizes between 0.2 and 0.5 were classified as small, between 0.5 and 0.8 as medium, and over 0.8 as large [22]. The following group comparisons were made: subjects with AD versus controls, subjects with $\mathrm{MCl}$ versus controls and subjects with $A D$ versus subjects with $\mathrm{MCl}$. We only performed pooled analyses if at least 3 independent studies contributed data for a specific combination of region and technique [23].

Heterogeneity was assessed with Cochrane's $Q$, which is calculated as the weighted sum of squared differences between individual study effects and the pooled effect across studies [24]. Since there was a significant level of heterogeneity between the studies, pooled Hedge's g was calculated using a random effects model [25]. Basic assumption of the random effects model is that the effects being estimated in the different studies are not identical, but follow some distribution around a mean global effect. We performed meta-analysis to identify possible explanations for heterogeneity. We tested the effect of subject characteristics (age, Mini Mental 
State Examination (MMSE) and Clinical Dementia Rating Scale (global score) (CDR)) and data acquisition parameters (MTL: field strength and slice thickness; DTI: field strength, b-value, voxel size, number of directions and number of excitations (NEX)) on Hedge's g using random effects meta-regression. Analyses were only performed if at least 6 studies provided data for the analysis.

Publication bias or the tendency of researchers to handle the reporting of experimental results that are positive differently from those that are negative (or inconclusive), leads to bias in the overall published literature [26]. This bias may lead to an overestimation of observed effects. We tested for this with the Begg and Mazumdar adjusted rank correlation test [27] and with funnel plots.

\section{RESULTS}

\section{MTL atrophy}

In total 2544 articles assessing MTL structures in $A D$ and/or $M C l$ were found. Seventy-six studies investigated atrophy in the MTL region and were subsequently included with a total of 8122 participants: 2431 subjects with AD, 2515 subjects with $\mathrm{MCl}$ and 3176 controls. Subject characteristics of the included MTL atrophy studies are provided in Table 2. Technical specifications of the MTL atrophy studies can be found in Supplementary Table 1.

\section{MTL measurements}

For the comparison between subjects with $A D$ and controls, ES Hedge's g was significant for all 4 measures studied (Table 3 ). Effect sizes were all large, ranging from 1.32 (parahippocampal gyrus) to 1.98 (entorhinal cortex). When comparing subjects with $\mathrm{MCl}$ and controls, Hedge's g was again significant in all 4 measures investigated (Table 3). ES was large for entorhinal cortex volume (1.46), MTA-score (1.11) and hippocampal volume (0.89) and medium for parahippocampal gyrus volume (0.61). The comparison of subjects with $\mathrm{MCl}$ and $\mathrm{AD}$ was significant for all 4 measures (Table 3). ES was large for entorhinal cortex (1.17), hippocampus (0.93) and parahippocampal gyrus (0.92) and medium for MTA-score (0.74).

\section{Heterogeneity and meta-regression}

Nine of 12 effect sizes showed a significant level of heterogeneity (Table 3). Fixed effects meta-regression showed no significant associations between ES and subject 
characteristics or data acquisition parameters.

\section{Publication bias}

Four of 12 group comparisons (controls vs AD; entorhinal cortex and hippocampal volume, controls vs $\mathrm{MCl}$; entorhinal cortex, $\mathrm{MCl}$ vs $\mathrm{AD}$; hippocampal volume) displayed a significant level of publication bias as measured by Begg and Mazumdar's rank correlations (Table 3).

\section{$D T I$}

In total 415 articles on DTI in $A D$ and/or $\mathrm{MCl}$ were found. Fifty-five studies were included in the meta-analysis with a total of 2791 participants: $819 \mathrm{AD}, 715 \mathrm{MCl}$ and 1257 controls. Subject characteristics of the included DTI studies are provided in Table 4. Technical specifications of the DTI studies can be found in Supplementary Table 2.

\section{Fractional anisotropy}

For the comparison between subjects with $A D$ and controls, the ES was significant in 10 of the 15 investigated regions (Table 5). A large ES was found in the total cingulum (1.73), parahippocampal cingulum (1.17), splenium of the corpus callosum (CC) (1.10), uncinate fasciculus (1.03) and posterior cingulum (0.84). Medium effect sizes were found in the SLF (0.77), frontal lobe $(0.70)$, genu CC (0.63), temporal lobe (0.57) and middle cingulum (0.42).

Between subjects with $\mathrm{MCl}$ and controls, Hedge's g was significant in 5 of the 12 regions studied (Table 5). A large ES was found in the parahippocampal cingulum (0.97) and a medium effect size in the posterior cingulum (0.65). A small ES was detected in splenium CC (0.44), genu CC (0.41) and temporal lobe (0.38). The comparison between subjects with $\mathrm{MCl}$ and $\mathrm{AD}$ was significant in 5 of the 8 regions studied: splenium CC (0.51), posterior cingulum (0.37), genu CC (0.35), frontal lobe (0.29) and temporal lobe (0.27). Effect sizes were all small (Table 5).

\section{Mean diffusivity}

For the comparison of $A D$ and controls, the ES was significant in all the 10 investigated regions (Table 6). A large ES was found in the hippocampus (-1.17), parietal lobe (-1.03), splenium CC (-0.94) and temporal lobe $(-0.89)$. A medium ES was detected in the frontal lobe $(-0.75)$, posterior cingulum $(-0.74)$, UF $(-0.72)$, genu 
CC (-0.67), occipital lobe (-0.67) and internal capsule (-0.66). When comparing $\mathrm{MCl}$ with controls, Hedge's g was significant in 7 of the 9 investigated regions (Table 5). A large ES was found in the hippocampus $(-1.00)$ and a medium ES in the parietal lobe (-0.69). A small ES was found in splenium CC (-0.46), genu CC (-0.43), temporal lobe $(-0.40)$, frontal lobe $(-0.32)$ and posterior cingulum $(-0.26)$. The comparison between subjects with $\mathrm{MCl}$ and $\mathrm{AD}$ was significant in 3 of the 5 regions studied: posterior cingulum (-0.40), genu CC (-0.32) and splenium CC (-0.04). All effect sizes were small (Table 5).

\section{Heterogeneity and meta-regression}

Twenty of 35 effect sizes for FA (Table 5) and 10 of 24 effect sizes for MD (Table 6) showed a significant level of heterogeneity. To investigate possible sources of heterogeneity, fixed effect regression was performed using patient characteristics (age, MMSE, CDR) and acquisition details (field strength, b-value, voxel size, number of directions and number of excitation). Meta-regression found some evidence that the ES was dependent on disease severity. In the comparison between controls and subjects with $A D$ ES increased with severity of the disease: ES of parietal lobe $F A(B=-0.1, p=0.04)$ and genu $C C M D(B=-0.2, p=0.01)$ increased with lower MMSE-scores. Comparing controls and $\mathrm{MCl}$-patients: $\mathrm{ES}$ of occipital lobe $\mathrm{MD}(\mathrm{B}=$ $-0.18, p<0.001)$ and hippocampal MD $(B=-0.5, p=0.02)$ increased with lower MMSEscores (Figure 1). Meta-regression furthermore indicated that a number of technical parameters were associated with ES (Supplementary Table 3).

\section{Publication bias}

Seven of 59 group comparisons for DTI displayed a significant level of publication bias, measured by Begg and Mazumdar's rank correlations. For FA, publication bias was present at comparisons between controls and $A D$ in frontal $W M$, genu $C C$, splenium $\mathrm{CC}$ and uncinate fasciculus, and between controls and $\mathrm{MCl}$ in splenium CC. For MD, the parietal lobe (controls vs $A D$ ) and the hippocampus (controls vs $\mathrm{MCl}$ ) showed a significant level of publication bias (Tables 5 and 6, Figure 2). 


\section{DISCUSSION}

The aim of the present study was to compare the value of structural MTL measurements with DTI modalities in discriminating controls, subjects with $\mathrm{MCl}$ and AD. Volumetric MTL atrophy measurements showed large effect sizes in all MTL regions investigated. Besides volumetric assessment of MTL regions, also diffusivity measurements seem to be sensitive for group differences in distinct brain regions, especially in temporal, parietal, cingulate and callosal regions.

\section{Group differences within and between MRI modalities}

For all group comparisons, the absolute ES of volumetric MTL atrophy measurements was somewhat larger than for the DTI measurements. However, for the comparison between controls and $\mathrm{MCl}$-patients, ES of hippocampal MD was larger than ES of hippocampal volume. This is in line with Müller [28] who stated that elevated diffusivity (MD) in hippocampal regions, particularly on the left side, was the strongest independent predictor of poor verbal memory performance in the total group of $\mathrm{MCl}$ patients and controls whereas hippocampus size could explain rather low proportions of variation in memory function.

As expected, for both structural and DTI measurements, ES for the differences between $A D$ and controls were larger than the ES for the differences between $\mathrm{MCl}$ / controls, and $\mathrm{MCl} / \mathrm{AD}$.

The largest difference in ES between MTL atrophy and DTI measurements was seen in the comparison between $\mathrm{MCl}$ and $\mathrm{AD}$. In this comparison, the DTI measurements had smaller ES than MTL atrophy measurements, indicating that DTI measurements showed more overlap between subjects with $\mathrm{MCl}$ and $\mathrm{AD}$ than MTL atrophy measurements. This could mean that in subjects with cognitive impairment loss of WM integrity precedes macroscopic atrophy. It could also be due to the fact that the difference in average MMSE score between subjects with $\mathrm{MCl}$ and $A D$ was slightly larger in the MTL atrophy studies (5.10) compared to the DTI studies (4.45), which may increase the ES for the MTL atrophy studies (see below).

There may be several explanations for the differences in ES between MTL atrophy and DTI measurements. First, there are more studies available investigating MTL atrophy compared to DTI, resulting in somewhat smaller ES and larger standard 
deviations for the DTI studies. Second, the ES is dependent on both the difference between the groups and the standard deviation and thus it is possible that in some regions DTI modalities have a somewhat lower ES due to a larger variability between subjects. Third, as mentioned above, subjects with $\mathrm{MCl}$ or AD included in MTL atrophy studies might have more severe cognitive impairment than subjects with $\mathrm{MCl}$ and $\mathrm{AD}$ included in DTI studies. However, we found no significant differences in severity of cognitive impairment in subjects with AD between MTL atrophy and DTI studies, as measured by the MMSE. For the MTL studies mean (sd) MMSE score for AD-patients was 21.41 (2.38) compared to 21.39 (3.04) for the AD-patients in the DTI studies $(p=0.85)$. Although it is possible that for specific comparisons there may be differences in disease severity (see above), it seems unlikely that the differences in ES between MTL and DTI studies are in general attributable to differences in disease severity between the MTL atrophy and DTI studies.

\section{Topographic differences}

DTI abnormalities were found in many different regions. FA and MD changes seem to follow a slightly different anatomical pattern. Highest ES (relative to controls) for DTI-FA were typically seen in cingulate and callosal areas (total cingulum, posterior cingulum, parahippocampal cingulum, splenium $\mathrm{CC}$ and uncinate fasciculus). For DTI-MD the largest differences were seen in the hippocampus, followed by the parietal lobe, splenium CC and temporal lobe. In general effect sizes of DTI-FA were comparable to those of DTI-MD. Smaller effect sizes in DTI-FA compared to DTI-MD were most notable in frontal, parietal, occipital and temporal lobe. This could be due to the fact that in regions with a more complex architecture, FA values are more variable due to a decrease in crossing fibers or other non-parallel organization [15]. It furthermore seems that the discriminative power of DTI-MD is slightly higher than for DTI-FA, with a broader set of regions showing significant white matter abnormalities. This finding is in line with the current literature stating that DTI-FA is a less sensitive marker for decreased white matter integrity than DTI-MD [29-31].

MTL atrophy was assessed in different ways and data suggest that entorhinal cortex (ERC) volume is the best volumetric discriminator as ERC showed the largest ES for all group comparisons. These results are in line with previous histological studies, which showed that the earliest neuropathological changes in AD appear in the ERC [32-34]. Furthermore, several MRI studies have suggested that volumetric measurements of 
the ERC are more sensitive than hippocampal volume for predicting $A D$ in subjects with $\mathrm{MCl}$ [35-37]. Structural MR studies also revealed abnormalities in $A D$ or $\mathrm{MCl}$ outside the MTL region. Callosal atrophy [38-41], cingulum atrophy [33, 42, 43], parietal [44] and frontal atrophy [45] have all been reported to be abnormal in $\mathrm{MCl}$ or AD.

\section{Clinical implications}

Our findings suggest that DTI may have the potential to be used in clinical practice in a similar way as MTL measurements. However, it is less likely that DTI will soon replace MTL atrophy assessment. First, there is limited information on how well DTI can discriminate subjects with AD from subjects with other types of dementia. At present there are insufficient studies available to provide pooled estimates for these differences. Preliminary findings suggest that FA values of the anterior WM are decreased in vascular dementia as well [46]. Another study [47] found that subjects with Lewy Body Dementia showed abnormalities in the corpus callosum, pericallosal areas and the frontal, parietal, occipital and, less prominently, in temporal white matter. Subjects with frontotemporal dementia (FTD) had greater reductions of FA in frontal brain regions relative to $A D$, whereas no other region in $A D$ showed greater reductions of FA when compared to FTD [48]. Second, longitudinal DTI-studies on the prediction of $A D$ in non-demented subjects are currently lacking. In the case of MTL measurements, studies indicate that MTL atrophy is a strong predictor for $A D$ in subjects with $\mathrm{MCl}$ with an ES ranging from 0.54 to 2.25 [9]. Third, to date DTI measurements generally provided group differences rather than dichotomized values that can be used in clinical practice. Sensitive cut-off scores for different DTI-modalities in distinct brain regions need to be determined and validated.

\section{Limitations}

The present study had several limitations. With regard to some brain structures our meta-analysis was based on relatively few studies, which reduced the accuracy of the parameter estimates. Moreover, as analyses of the different techniques were based on different studies, we cannot exclude the possibility that differences in ES resulted from differences in sample selection and other study characteristics. We frequently observed statistically significant heterogeneity between the studies. Meta-regression could only explain part of this heterogeneity. Moreover, for some parameters, the number of studies available for meta-regression analyses was small, 
which limited statistical power. In general slice thickness in DTI studies was higher than in MTL studies. This factor might have reduced sensitivity of DTI markers. The only outcome measure calculated in this study is the ES. Accuracy measurements such as sensitivity or specificity could not be taken into account since dichotomous data of the included studies was not available. One author [30] reported a sensitivity up to $89 \%$ for $\mathrm{MD}$ and $78 \%$ for FA measurements for the detection of hippocampal abnormalities in subjects with $\mathrm{MCl}$. For a number of regions and comparisons, data had been published in less than 3 studies and could therefore not be included in the meta-analysis. For example, only a few studies investigated DTI in the entorhinal cortex or the perforant pathway. Furtermore, it was only possible to examine FA differences in all cingulum regions between controls and subjects with AD. Superior longitudinal fasciculus, uncinate fasciculus and internal capsule could also not be examined for all group comparisons. Our results may have been affected by publication bias and by the fact that not all studies presented means and standard deviations for the regions studied or did not reply to our request for data. However, it is unlikely that publication bias substantially influenced our results since Begg and Mazumdar's rank correlation was only significant $(p<.05)$ for 4 group comparison in the MTL studies and 7 group comparisons in the DTI studies. Furthermore, a number of these significant comparisons could be due to multiple testing effects since only one of these comparisons was significant at $p<.01$. We limited our meta-analysis to FA and MD. However, it has recently been suggested that the examination of directional diffusivities such as axial (DA) and radial diffusion (DR) may yield important information about the underlying neuropathology driving differences in FA as well [49-52]. One author suggested that DA and DR may be even more reliable markers of degeneration than FA [29]. Still, there are not enough studies to conduct a separate meta-analysis of these modalities. It has to be noted that in many studies FA values in the hippocampal region, which mainly consists of gray matter, are usually ignored. Mean FA values in the hippocampus are generally lower than 0.2 , which is close to the noise level. Hippocampal FA therefore needs to be interpreted with caution. 


\section{CONCLUSION}

Despite the fact that one study suggested that DTI measurements seem to be more sensitive than volumetric measurements [30] or that measures of microstructure provide unique information not obtainable with volumetric mapping in regions known to be crucial in AD [53], our review does not support the notion that DTI is superior to structural MTL assessment in detecting early stage AD. Nonetheless, based on the current literature [19] and our findings, we can conclude that DTI is a sensitive method to detect white matter changes in subjects with $\mathrm{MCl}$ and $\mathrm{AD}$. It seems that in widespread brain regions diffusivity is already impaired early in the disease process, with more severe white matter disruptions with increasing disease severity. There is a need of additional studies that test the predictive accuracy of DTI measurements for $A D$ in subjects with $\mathrm{MCl}$ and that test differences in DTI measurements between $A D$ and other dementias. In order to better understand the development of WM integrity loss in relation to grey matter loss and the diagnostic potential of DTI measurements, there is a particular need for longitudinal MRI studies that combine structural with DTI assessments. Such studies will advance our understanding of the effects of neurodegeneration on gray matter, white matter, and their interaction and will enhance our ability to detect subtle brain changes early in the disease process. Since diagnostic criteria for prodromal AD require the presence of episodic memory impairment in combination with at least one $A D$ biomarker [5, 54], future studies should compare the use of DTI makers for the diagnosis of prodromal $A D$ with that of other $A D$ biomarkers such as CSF markers [55, 56], PET imaging markers [57] or familial genetic mutations [58]. 


\section{REFERENCES}

1. Barnes DE, Yaffe K. The projected effect of risk factor reduction on Alzheimer's disease prevalence. The Lancet Neurology 2011;10:819-828.

2. Jack $C R$, Shiung $M M$, Weigand SD, et al. Brain atrophy rates predict subsequent clinical conversion in normal elderly and amnestic $\mathrm{MCl}$. Neurology 2005;65:1227-1231.

3. Petersen RC, Smith GE, Waring SC, Ivnik RJ, Tangalos EG, Kokmen E. Mild Cognitive Impairment: Clinical Characterization and Outcome. Archives of Neurology 1999;56:303-308.

4. Bruscoli $\mathrm{M}$, Lovestone $\mathrm{S}$. Is $\mathrm{MCl}$ really just early dementia? A systematic review of conversion studies. International Psychogeriatrics 2004;16:129-140.

5. Dubois B, Feldman HH, Jacova C, et al. Research criteria for the diagnosis of Alzheimer's disease: revising the NINCDS-ADRDA criteria. The Lancet Neurology 2007;6:734-746.

6. Rusinek HP, Endo YM, De Santi SP, et al. Atrophy rate in medial temporal lobe during progression of Alzheimer disease. Neurology 2004;63:2354-2359.

7. Schoonenboom NSM, van der Flier WM, Blankenstein MA, et al. CSF and MRI markers independently contribute to the diagnosis of Alzheimer's disease. Neurobiology of Aging 2008;29:669-675.

8. Visser PJ, Scheltens P, Verhey FRJ, et al. Medial temporal lobe atrophy and memory dysfunction as predictors for dementia in subjects with mild cognitive impairment. Journal of Neurology 1999;246:477-485.

9. Schmand B, Huizenga HM, van Gool WA. Meta-analysis of CSF and MRI biomarkers for detecting preclinical Alzheimer's disease. Psychological Medicine 2010;40:135-145.

10. Medina D, deToledo-Morrell L, Urresta F, et al. White matter changes in mild cognitive impairment and AD: A diffusion tensor imaging study. Neurobiology of Aging 2006;27:663-672.

11. Wang L, Goldstein FC, Veledar E, et al. Alterations in Cortical Thickness and White Matter Integrity in Mild Cognitive Impairment Measured by WholeBrain Cortical Thickness Mapping and Diffusion Tensor Imaging. AJNR. American Journal of Neuroradiology 2009;30:893-899.

12. Smith SM, Johansen-Berg $\mathrm{H}$, Jenkinson $\mathrm{M}$, et al. Acquisition and voxelwise analysis of multi-subject diffusion data with Tract-Based Spatial Statistics. Nat. Protocols 2007;2:499-503. 
13. Pierpaoli C, Jezzard P, Basser PJ, Barnett A, Di Chiro G. Diffusion tensor MR imaging of the human brain. Radiology 1996;201:637-648.

14. Basser PJ, Mattiello J, Lebihan D. Estimation of the Effective Self-Diffusion Tensor from the NMR Spin Echo. Journal of Magnetic Resonance, Series B 1994;103:247-254.

15. Stebbins GT, Murphy CM. Diffusion tensor imaging in Alzheimer's disease and mild cognitive impairment, 2009.

16. Nakata $\mathrm{Y}$, Sato $\mathrm{N}, \mathrm{Abe} \mathrm{O}$, et al. Diffusion abnormality in posterior cingulate fiber tracts in Alzheimer's disease: tract-specific analysis. Radiation Medicine 2008;26:466-473.

17. Bozzali M, Cherubini A. Diffusion tensor MRI to investigate dementias: a brief review. Magnetic Resonance Imaging 2007;25:969-977.

18. Medina DA, Gaviria M. Diffusion tensor imaging investigations in Alzheimer's disease: the resurgence of white matter compromise in the cortical dysfunction of the aging brain. Neuropsychiatr Dis Treat 2008;4.

19. Sexton CE, Kalu UG, Filippini N, Mackay CE, Ebmeier KP. A meta-analysis of diffusion tensor imaging in mild cognitive impairment and Alzheimer's disease. Neurobiology of Aging 2010;32.

20. Scheltens P, Leys D, Barkhof F, et al. Atrophy of medial temporal lobes on MRI in "probable" Alzheimer's disease and normal ageing: diagnostic value and neuropsychological correlates. Journal of Neurology, Neurosurgery and Psychiatry 1992;55:967-972.

21. Hedges LV. Distribution Theory for Glass's Estimator of Effect size and Related Estimators. Journal of Educational and Behavioral Statistics 1981;6:107-128.

22. Cohen J. Statistical Power Analysis for the Behavioral Sciences, Second ed: Lawrence Erlbaum Associates, Hillsdale, New Jersey, USA., 1988.

23. Kempton MJ, Geddes JR, Ettinger U, Williams SCR, Grasby PM. Meta-analysis, Database, and Meta-regression of 98 Structural Imaging Studies in Bipolar Disorder. Archives of General Psychiatry 2008;65:1017-1032.

24. Gavaghan DJ, Moore RA, McQuay HJ. An evaluation of homogeneity tests in meta-analyses in pain using simulations of individual patient data. Pain 2000;85:415-424.

25. DerSimonian R, Laird N. Meta-analysis in clinical trials. Controlled Clinical Trials 1986;7:177-188.

26. Easterbrook PJ, Gopalan R, Berlin JA, Matthews DR. Publication bias in clinical 
research. The Lancet 1991;337:867-872.

27. Begg CB, Mazumdar M. Operating Characteristics of a Rank Correlation Test for Publication Bias. Biometrics 1994;50:1088-1101.

28. Müller MJ, Greverus D, Dellani PR, et al. Functional implications of hippocampal volume and diffusivity in mild cognitive impairment. Neuroimage 2005;28:1033-1042.

29. Acosta-Cabronero J, Williams GB, Pengas G, Nestor PJ. Absolute diffusivities define the landscape of white matter degeneration in Alzheimer's disease. Brain 2010;133:529-539.

30. Müller MJ, Greverus D, Weibrich C, et al. Diagnostic utility of hippocampal size and mean diffusivity in amnestic MCl. Neurobiology of Aging 2007;28:398403.

31. Sexton CE, Mackay CE, Lonie JA, et al. MRI correlates of episodic memory in Alzheimer's disease, mild cognitive impairment, and healthy aging. Psychiatry Research: Neuroimaging 2010;184:57-62.

32. Braak H, Braak E. Neurofibrillary changes confined to the entorhinal region and an abundance of cortical amyloid in cases of presenile and senile dementia. Acta Neuropathologica 1990;80:479-486.

33. Choo ILH, Lee DY, Oh JS, et al. Posterior cingulate cortex atrophy and regional cingulum disruption in mild cognitive impairment and Alzheimer's disease. Neurobiology of Aging 2010;31:772-779.

34. Van Hoesen GW. The parahippocampal gyrus: New observations regarding its cortical connections in the monkey. Trends in Neurosciences 1982;5:345-350.

35. deToledo-Morrell L, Stoub TR, Bulgakova M, et al. MRI-derived entorhinal volume is a good predictor of conversion from $\mathrm{MCl}$ to $A D$. Neurobiology of Aging 2004;25:1197-1203.

36. Dickerson BC, Goncharova I, Sullivan MP, et al. MRI-derived entorhinal and hippocampal atrophy in incipient and very mild Alzheimer's disease. Neurobiology of Aging 2001;22:747-754.

37. Pennanen C, Kivipelto M, Tuomainen S, et al. Hippocampus and entorhinal cortex in mild cognitive impairment and early AD. Neurobiology of Aging 2004;25:303-310.

38. Di Paola M, Di lulio F, Cherubini A, et al. When, where, and how the corpus callosum changes in $\mathrm{MCl}$ and $\mathrm{AD}$ : A multimodal MRI study. Neurology 2010;74:1136-1142.

142 | Chapter 5: Meta-analysis DTI and MTL measurements 
39. Chen $T-F$, Lin $C-C$, Chen $Y-F$, et al. Diffusion tensor changes in patients with amnesic mild cognitive impairment and various dementias. Psychiatry Research: Neuroimaging 2009;173:15-21.

40. Serra L, Cercignani M, Lenzi D, et al. Grey and White Matter Changes at Different Stages of Alzheimer's Disease. Journal of Alzheimer's Disease 2010;19:147-159.

41. Wang H, Su MY. Regional Pattern of Increased Water Diffusivity in Hippocampus and Corpus Callosum in Mild Cognitive Impairment. Dementia and Geriatric Cognitive Disorders 2006;22:223-229.

42. Callen DJA, Black SE, Gao F, Caldwell CB, Szalai JP. Beyond the hippocampus: MRI volumetry confirms widespread limbic atrophy in AD. Neurology 2001;57:1669-1674.

43. Jones BF, Barnes J, Uylings HBM, et al. Differential Regional Atrophy of the Cingulate Gyrus in Alzheimer Disease: A Volumetric MRI Study. Cerebral Cortex 2006;16:1701-1708.

44. Jacobs HIL, Van Boxtel MPJ, Jolles J, Verhey FRJ, Uylings HBM. Parietal cortex matters in Alzheimer's disease: An overview of structural, functional and metabolic findings. Neuroscience and Biobehavioral Reviews 2011.

45. Burgmans S, van Boxtel MPJ, Smeets F, et al. Prefrontal cortex atrophy predicts dementia over a six-year period. Neurobiology of Aging 2009;30:1413-1419.

46. Sugihara S, Kinoshita T, Matsusue E, Fujii S, Ogawa T. Usefulness of Diffusion Tensor Imaging of White Matter in Alzheimer Disease and Vascular Dementia. Acta Radiologica 2004;45:658-663.

47. Bozzali M, Falini A, Cercignani M, et al. Brain tissue damage in dementia with Lewy bodies: an in vivo diffusion tensor MRI study. Brain 2005;128:15951604.

48. Zhang $Y$, Schuff $N$, Du A-T, et al. White matter damage in frontotemporal dementia and Alzheimer's disease measured by diffusion MRI. Brain 2009;132:2579-2592.

49. Bosch B, Arenaza-Urquijo EM, Rami L, et al. Multiple DTI index analysis in normal aging, amnestic $\mathrm{MCl}$ and $\mathrm{AD}$. Relationship with neuropsychological performance. Neurobiology of Aging 2012;33:61-74.

50. Salat DH, Tuch DS, van der Kouwe AJW, et al. White matter pathology isolates the hippocampal formation in Alzheimer's disease. Neurobiology of Aging 2010;31:244-256. 
51. Song S-K, Sun S-W, Ju W-K, Lin S-J, Cross AH, Neufeld AH. Diffusion tensor imaging detects and differentiates axon and myelin degeneration in mouse optic nerve after retinal ischemia. Neuroimage 2003;20:1714-1722.

52. Sun S-W, Song S-K, Harms MP, et al. Detection of age-dependent brain injury in a mouse model of brain amyloidosis associated with Alzheimer's disease using magnetic resonance diffusion tensor imaging. Experimental Neurology 2005;191:77-85.

53. Canu E, McLaren DG, Fitzgerald ME, et al. Microstructural Diffusion Changes are Independent of Macrostructural Volume Loss in Moderate to Severe Alzheimer's Disease. Journal of Alzheimer's Disease 2010;19:963-976.

54. Dubois B, Feldman HH, Jacova C, et al. Revising the definition of Alzheimer's disease: a new lexicon. The Lancet Neurology 2010;9:1118-1127.

55. Motter R, Vigo-Pelfrey $C$, Kholodenko $D$, et al. Reduction of $\beta$-amyloid peptide42 in the cerebrospinal fluid of patients with Alzheimer's disease. Annals of Neurology 1995;38:643-648.

56. Vandermeeren M, Mercken M, Vanmechelen E, et al. Detection of Proteins in Normal and Alzheimer's Disease Cerebrospinal Fluid with a Sensitive Sandwich Enzyme-Linked Immunosorbent Assay. Journal of Neurochemistry 1993;61:1828-1834.

57. Coleman RE. Positron Emission Tomography Diagnosis of Alzheimer's Disease. Neuroimaging Clinics of North America 2005;15:837-846.

58. Bird TDM. Genetic aspects of Alzheimer disease. Genetics in Medicine 2005;10:231-239.

59. Pitkanen A, Laakso $M$, Kalviainen $R$, et al. Severity of hippocampal atrophy correlates with the prolongation of MRI T sub 2 relaxation time in temporal lobe epilepsy but not in Alzheimer's disease. Neurology 1996;46:1724-1730.

60. Convit A, De Leon MJ, Tarshish C, et al. Specific Hippocampal Volume Reductions in Individuals at Risk for Alzheimer's Disease. Neurobiology of Aging 1997;18:131-138.

61. Kaye JA, Swihart T, Howieson D, et al. Volume loss of the hippocampus and temporal lobe in healthy elderly persons destined to develop dementia. Neurology 1997;48:1297-1304.

62. Mori E, Yoneda $\mathrm{Y}$, Yamashita H, Hirono N, Ikeda M, Yamadori A. Medial temporal structures relate to memory impairment in Alzheimer's disease: an MRI volumetric study. Journal of Neurology, Neurosurgery and Psychiatry 
1997;63:214-221.

63. Jack CR, Jr., Petersen RC, Xu Y, et al. Rate of medial temporal lobe atrophy in typical aging and Alzheimer's disease. Neurology 1998;51:993-999.

64. Juottonen K, Laakso MP, Insausti R, et al. Volumes of the Entorhinal and Perirhinal Cortices in Alzheimer's Disease. Neurobiology of Aging 1998;19:1522.

65. Krasuski JS, Alexander GE, Horwitz B, et al. Volumes of medial temporal lobe structures in patients with Alzheimer's disease and mild cognitive impairment (and in healthy controls). Biological Psychiatry 1998;43:60-68.

66. Laakso MP, Soininen $\mathrm{H}$, Partanen $\mathrm{K}$, et al. MRI of the Hippocampus in Alzheimer's Disease: Sensitivity, Specificity, and Analysis of the Incorrectly Classified Subjects. Neurobiology of Aging 1998;19:23-31.

67. Barber R, McKeith IG, Ballard C, Gholkar A, O'Brien JT. A Comparison of Medial and Lateral Temporal Lobe Atrophy in Dementia with Lewy Bodies and Alzheimer's Disease: Magnetic Resonance Imaging Volumetric Study. Dementia and Geriatric Cognitive Disorders 2000;12:198-205.

68. De Toledo-Morrell L, Goncharova I, Dickerson B, Wilson RS, Bennett DA. From Healthy Aging to Early Alzheimer's Disease: In Vivo Detection of Entorhinal Cortex Atrophy. Annals of the New York Academy of Sciences 2000;911:240253.

69. Laakso MP, Frisoni GB, Könönen M, et al. Hippocampus and entorhinal cortex in frontotemporal dementia and Alzheimer's disease: a morphometric MRI study. Biological Psychiatry 2000;47:1056-1063.

70. Xu Y, Jack CR, O'Brien PC, et al. Usefulness of MRI measures of entorhinal cortex versus hippocampus in AD. Neurology 2000;54:1760-1767.

71. De Santi S, de Leon MJ, Rusinek $\mathrm{H}$, et al. Hippocampal formation glucose metabolism and volume losses in $\mathrm{MCl}$ and $\mathrm{AD}$. Neurobiology of Aging 2001;22:529-539.

72. Du AT, Schuff N, Amend D, et al. Magnetic resonance imaging of the entorhinal cortex and hippocampus in mild cognitive impairment and Alzheimer's disease. Journal of Neurology, Neurosurgery and Psychiatry 2001;71:441447.

73. Goncharova II, Dickerson BC, Stoub TR, deToledo-Morrell L. MRI of human entorhinal cortex: a reliable protocol for volumetric measurement. Neurobiology of Aging 2001;22:737-745. 
74. Wolf $H$, Grunwald $M$, Kruggel $F$, et al. Hippocampal volume discriminates between normal cognition; questionable and mild dementia in the elderly. Neurobiology of Aging 2001;22:177-186.

75. Bottino C, aacute, ssio MC, et al. Volumetric MRI Measurements Can Differentiate Alzheimer's Disease, Mild Cognitive Impairment, and Normal Aging. International Psychogeriatrics 2002;14:59-72.

76. Hsu Y-Y, Schuff N, Du A-T, et al. Comparison of automated and manual MRI volumetry of hippocampus in normal aging and dementia. Journal of Magnetic Resonance Imaging 2002;16:305-310.

77. Killiany RJ, Hyman BT, Gomez-Isla T, et al. MRI measures of entorhinal cortex vs hippocampus in preclinical AD. Neurology 2002;58:1188-1196.

78. Mega MS, Small GW, Xu ML, et al. Hippocampal Atrophy in Persons With Age-Associated Memory Impairment: Volumetry Within a Common Space. Psychosomatic Medicine 2002;64:487-492.

79. Pantel J, Häger DR, Kratz B, et al. Strukturelle zerebrale Veranderungen bei Probanden mit leichter kognitiver Beeintrachtigung Eine MR-volumetrische Studie. Der Nervenarzt 2002;73:845-850.

80. Du AT, Schuff $N$, Zhu XP, et al. Atrophy rates of entorhinal cortex in $A D$ and normal aging. Neurology 2003;60:481-486.

81. Du AT, Schuff N, Kramer JH, et al. Higher atrophy rate of entorhinal cortex than hippocampus in AD. Neurology 2004;62:422-427.

82. Testa C, Laakso MP, Sabattoli F, et al. A comparison between the accuracy of voxel-based morphometry and hippocampal volumetry in Alzheimer's disease. Journal of Magnetic Resonance Imaging 2004;19:274-282.

83. Bastos-Leite AJ, van Waesberghe JH, Oen AL, van der Flier WM, Scheltens P, Barkhof F. Hippocampal Sulcus Width and Cavities: Comparison Between Patients with Alzheimer Disease and Nondemented Elderly Subjects. AJNR. American Journal of Neuroradiology 2006;27:2141-2145.

84. Kalus P, Slotboom J, Gallinat Jr, et al. Examining the gateway to the limbic system with diffusion tensor imaging: The perforant pathway in dementia. Neuroimage 2006;30:713-720.

85. Teipel S, Pruessner J, Faltraco F, et al. Comprehensive dissection of the medial temporal lobe in AD: measurement of hippocampus, amygdala, entorhinal, perirhinal and parahippocampal cortices using MRI. Journal of Neurology 2006;253:794-800. 
86. Uotani C, Sugimori K, Kobayashi K. Association of minimal thickness of the medial temporal lobe with hippocampal volume, maximal and minimal hippocampal length: Volumetric approach with horizontal magnetic resonance imaging scans for evaluation of a diagnostic marker for neuroimaging of Alzheimer's disease. Psychiatry and Clinical Neurosciences 2006;60:319-326.

87. van de Pol LA, Hensel A, van der Flier WM, et al. Hippocampal atrophy on MRI in frontotemporal lobar degeneration and Alzheimer disease. Journal of Neurology, Neurosurgery and Psychiatry 2006;77:439-442.

88. Barnes J, Godbolt AK, Frost C, et al. Atrophy rates of the cingulate gyrus and hippocampus in AD and FTLD. Neurobiology of Aging 2007;28:20-28.

89. Meyer JS, Huang J, Chowdhury MH. MRI confirms mild cognitive impairments prodromal for Alzheimer's, vascular and Parkinson-Lewy body dementias. Journal of the Neurological Sciences 2007;257:97-104.

90. Ridha BH, Barnes J, van de Pol LA, et al. Application of Automated Medial Temporal Lobe Atrophy Scale to Alzheimer Disease. Archives of Neurology 2007;64:849-854.

91. Slavin MJ, Sandstrom CK, Tran T-TT, Doraiswamy PM, Petrella JR. Hippocampal Volume and the Mini-Mental State Examination in the Diagnosis of Amnestic Mild Cognitive Impairment. American Journal of Roentgenology 2007;188:1404-1410.

92. Colliot $\mathrm{O}$, Chételat $\mathrm{G}$, Chupin M, et al. Discrimination between Alzheimer Disease, Mild Cognitive Impairment, and Normal Aging by Using Automated Segmentation of the Hippocampus1. Radiology 2008;248:194-201.

93. Delano-Wood L, Bondi MW, Jak AJ, et al. Stroke risk modifies regional white matter differences in mild cognitive impairment. Neurobiology of Aging 2008;31:1721-1731.

94. Duara R, Loewenstein DA, Potter E, et al. Medial temporal lobe atrophy on MRI scans and the diagnosis of Alzheimer disease. Neurology 2008;71:19861992.

95. Kenny ER, Burton EJ, O'Brien JT. A volumetric magnetic resonance imaging study of entorhinal cortex volume in dementia with lewy bodies. A comparison with Alzheimer's disease and Parkinson's disease with and without dementia. Dementia and Geriatric Cognitive Disorders 2008;26:218-225.

96. Appel J, Potter E, Bhatia N, et al. Association of White Matter Hyperintensity Measurements on Brain MR Imaging with Cognitive Status, Medial Temporal 
Atrophy, and Cardiovascular Risk Factors. American Journal of Neuroradiology 2009;30:1870-1876.

97. Bai F, Zhang Z, Watson DR, et al. Absent gender differences of hippocampal atrophy in amnestic type mild cognitive impairment. Neuroscience Letters 2009;450:85-89.

98. Cho H, Kwon J-H, Seo H-J. Medial temporal lobe atrophy in vascular dementia: Visual temporal lobe rating scale. Archives of Gerontology and Geriatrics 2009;48:415-418.

99. Feczko E, Augustinack JC, Fischl B, Dickerson BC. An MRI-based method for measuring volume, thickness and surface area of entorhinal, perirhinal, and posterior parahippocampal cortex. Neurobiology of Aging 2009;30:420-431.

100. Ferrarini L, Frisoni GB, Pievani M, Reiber JHC, Ganzola R, Milles J. Morphological Hippocampal Markers for Automated Detection of Alzheimer's Disease and Mild Cognitive Impairment Converters in Magnetic Resonance Images. Journal of Alzheimer's Disease 2009;17:643-659.

101. Henneman WJP, Sluimer JD, Barnes J, et al. Hippocampal atrophy rates in Alzheimer disease: Added value over whole brain volume measures. Neurology 2009;72:999-1007.

102. Henneman WJP, Vrenken $H$, Barnes J, et al. Baseline CSF p-tau levels independently predict progression of hippocampal atrophy in Alzheimer disease. Neurology 2009;73:935-940.

103. Hyun C, Jee-Hyun K, Hyun-Jin S. Medial temporal lobe atrophy in vascular dementia: Visual temporal lobe rating scale. Archives of Gerontology and Geriatrics 2009;48:415-418.

104. Jauhiainen $A M$, Pihlajamäki $M$, Tervo $S$, et al. Discriminating accuracy of medial temporal lobe volumetry and $\mathrm{FMRI}$ in mild cognitive impairment. Hippocampus 2009;19:166-175.

105. Lee DY, Fletcher E, Martinez $\mathrm{O}$, et al. Regional pattern of white matter microstructural changes in normal aging, $\mathrm{MCl}$, and AD. Neurology 2009;73:1722-1728.

106. Loewenstein DA, Acevedo A, Potter E, et al. Severity of Medial Temporal Atrophy and Amnestic Mild Cognitive Impairment: Selecting Type and Number of Memory Tests. American Journal of Geriatric Psych 2009;17:10501058.

107. Morra JH, Tu Z, Apostolova LG, et al. Automated 3D mapping of hippocampal 148 | Chapter 5: Meta-analysis DTI and MTL measurements 
atrophy and its clinical correlates in 400 subjects with Alzheimer's disease, mild cognitive impairment, and elderly controls. Human Brain Mapping 2009;30:2766-2788.

108. Rogalski EJ, Murphy CM, deToledo-Morrell L, et al. Changes in parahippocampal white matter integrity in amnestic mild cognitive impairment: A diffusion tensor imaging study. Behavioural Neurology 2009;21:51-61.

109. Bird CM, Chan D, Hartley T, Pijnenburg YA, Rossor MN, Burgess N. Topographical short-term memory differentiates Alzheimer's disease from frontotemporal lobar degeneration. Hippocampus 2010;20:1154-1169.

110. Bouwman FH, Verwey NA, Klein M, et al. New Research Criteria for the Diagnosis of Alzheimer's Disease Applied in a Memory Clinic Population. Dementia and Geriatric Cognitive Disorders 2010;30:1-7.

111. Cherubini A, Péran P, Spoletini I, et al. Combined Volumetry and DTI in Subcortical Structures of Mild Cognitive Impairment and Alzheimer's Disease Patients. Journal of Alzheimer's Disease 2010;19:1273-1282.

112. Desikan RS, Sabuncu MR, Schmansky NJ, et al. Selective disruption of the cerebral neocortex in Alzheimer's disease. PLoS One 2010;5:e12853-e12853.

113. Jhoo JH, Lee DY, Choo $\mathrm{IH}$, et al. Discrimination of normal aging, $\mathrm{MCl}$ and $\mathrm{AD}$ with multimodal imaging measures on the medial temporal lobe. Psychiatry Research: Neuroimaging 2010;183:237-243.

114. Johnson DK, Barrow W, Anderson R, et al. Diagnostic Utility of Cerebral White Matter Integrity in Early Alzheimer's Disease. International Journal of Neuroscience 2010;120:544-550.

115. Lehmann M, Douiri A, Kim LG, et al. Atrophy patterns in Alzheimer's disease and semantic dementia: A comparison of FreeSurfer and manual volumetric measurements. Neuroimage 2010;49:2264-2274.

116. Liu Y, Paajanen T, Zhang $\mathrm{Y}$, et al. Analysis of regional MRI volumes and thicknesses as predictors of conversion from mild cognitive impairment to Alzheimer's disease. Neurobiology of Aging 2010;In Press, Corrected Proof.

117. Luckhaus C, Jänner M, Cohnen M, et al. A novel MRI-biomarker candidate for Alzheimer's disease composed of regional brain volume and perfusion variables. European Journal of Neurology 2010;17:1437-1444.

118. Mueller SG, Schuff N, Yaffe K, Madison C, Miller B, Weiner MW. Hippocampal atrophy patterns in mild cognitive impairment and Alzheimer's disease. Human Brain Mapping 2010;31:1339-1347. 
119. Pengas $G$, Hodges JR, Watson P, Nestor PJ. Focal posterior cingulate atrophy in incipient Alzheimer's disease. Neurobiology of Aging 2010;31:25-33.

120. Ryu SY, Kwon MJ, Lee SB, et al. Measurement of precuneal and hippocampal volumes using magnetic resonance volumetry in Alzheimer's disease. Journal of clinical neurology (Seoul, Korea) 2010;6:196-203.

121. Sanchez-Benavides G, Gomez-Anson B, Molinuevo JL, et al. Medial Temporal Lobe Correlates of Memory Screening Measures in Normal Aging, $\mathrm{MCl}$, and AD. Journal of Geriatric Psychiatry and Neurology 2010;23:100-108.

122. Schott JM, Bartlett JW, Barnes J, Leung KK, Ourselin S, Fox NC. Reduced sample sizes for atrophy outcomes in Alzheimer's disease trials: baseline adjustment. Neurobiology of Aging 2010;31:1452-1462.e1452.

123. Westman E, Wahlund L-O, Foy C, et al. Combining MRI and MRS to Distinguish Between Alzheimer's Disease and Healthy Controls. Journal of Alzheimer's Disease 2010;22:171-181.

124. Yakushev I, Müller MJ, Lorscheider M, et al. Increased hippocampal head diffusivity predicts impaired episodic memory performance in early Alzheimer's disease. Neuropsychologia 2010;48:1447-1453.

125. Zarei M, Patenaude B, Damoiseaux J, et al. Combining shape and connectivity analysis: An MRI study of thalamic degeneration in Alzheimer's disease. Neuroimage 2010;49:1-8.

126. Echavarri $\mathrm{C}$, Aalten $\mathrm{P}$, Uylings $\mathrm{H}$, et al. Atrophy in the parahippocampal gyrus as an early biomarker of Alzheimer's disease. Brain Structure and Function 2011;215:265-271.

127. Prestia A, Boccardi M, Galluzzi S, et al. Hippocampal and amygdalar volume changes in elderly patients with Alzheimer's disease and schizophrenia. Psychiatry Research: Neuroimaging 2011;192:77-83.

128. Zhang Y-Z, Chang C, Wei X-e, Fu J-L, Li W-B. Comparison of diffusion tensor image study in association fiber tracts among normal, amnestic mild cognitive impairment, and Alzheimer's patients. Neurology India 2011;59:168-173.

129. McKhann G, Drachman D, Folstein M, Katzman R, Price D, Stadlan EM. Clinical diagnosis of Alzheimer's disease. Neurology 1984;34:939-939.

130. Petersen RC, Doody R, Kurz A, et al. Current concepts in mild cognitive impairment. Archives of Neurology 2001;58:1985-1992.

131. Rose SE, Chen F, Chalk JB, et al. Loss of connectivity in Alzheimer's disease: an evaluation of white matter tract integrity with colour coded MR diffusion 
tensor imaging. Journal of Neurology, Neurosurgery and Psychiatry 2000;69:528-530.

132. Bozzali M, Falini A, Franceschi M, et al. White matter damage in Alzheimer's disease assessed in vivo using diffusion tensor magnetic resonance imaging. Journal of Neurology, Neurosurgery and Psychiatry 2002;72:742-746.

133. Stahl R, Dietrich O, Teipel S, Hampel H, Reiser MF, Schoenberg SO. Diffusion tensor imaging zur Erfassung axonaler Degeneration bei Morbus Alzheimer. Der Radiologe 2003;43:566-575.

134. Fellgiebel A, Wille P, Müller MJ, et al. Ultrastructural Hippocampal and White Matter Alterations in Mild Cognitive Impairment: A Diffusion Tensor Imaging Study. Dementia and Geriatric Cognitive Disorders 2004;18:101-108.

135. Head D, Buckner RL, Shimony JS, et al. Differential Vulnerability of Anterior White Matter in Nondemented Aging with Minimal Acceleration in Dementia of the Alzheimer Type: Evidence from Diffusion Tensor Imaging. Cerebral Cortex 2004;14:410-423.

136. Choi SJ, Lim KO, Monteiro I, Reisberg B. Diffusion Tensor Imaging of Frontal White Matter Microstructure in Early Alzheimer's Disease: A Preliminary Study. Journal of Geriatric Psychiatry and Neurology 2005;18:12-19.

137. Fellgiebel $A, M$, ller MJ, Wille $P$, et al. Color-coded diffusion-tensor-imaging of posterior cingulate fiber tracts in mild cognitive impairment. Neurobiology of Aging 2005;26:1193-1198.

138. Duan J-H, Wang $\mathrm{H}-\mathrm{Q}, \mathrm{Xu} \mathrm{J}$, et al. White matter damage of patients with Alzheimer's disease correlated with the decreased cognitive function. Surgical and Radiologic Anatomy 2006;28:150-156.

139. Naggara O, Oppenheim C, Rieu D, et al. Diffusion tensor imaging in early Alzheimer's disease. Psychiatry Research: Neuroimaging 2006;146:243-249.

140. Rose SE, McMahon KL, Janke AL, et al. Diffusion indices on magnetic resonance imaging and neuropsychological performance in amnestic mild cognitive impairment. Journal of Neurology, Neurosurgery and Psychiatry 2006;77:1122-1128.

141. Taoka T, Iwasaki S, Sakamoto M, et al. Diffusion Anisotropy and Diffusivity of White Matter Tracts Within the Temporal Stem in Alzheimer Disease: Evaluation of the "Tract of Interest" by Diffusion Tensor Tractography. American Journal of Neuroradiology 2006;27:1040-1045.

142. Chen S-q, Kang Z, Hu X-q, Hu B, Zou Y. Diffusion tensor imaging of the brain 
in patients with Alzheimer's disease and cerebrovascular lesions. Journal of Zhejiang University - Science B 2007;8:242-247.

143. Firbank MJ, Blamire AM, Krishnan MS, et al. Diffusion tensor imaging in dementia with Lewy bodies and Alzheimer's disease. Psychiatry Research: Neuroimaging 2007;155:135-145.

144. Huang J, Auchus AP. Diffusion Tensor Imaging of Normal Appearing White Matter and Its Correlation with Cognitive Functioning in Mild Cognitive Impairment and Alzheimer's Disease. Annals of the New York Academy of Sciences 2007;1097:259-264.

145. Stahl R, Dietrich O, Teipel SJ, Hampel H, Reiser MF, Schoenberg SO. White Matter Damage in Alzheimer Disease and Mild Cognitive Impairment: Assessment with Diffusion-Tensor MR Imaging and Parallel Imaging Techniques1. Radiology 2007;243:483-492.

146. Zhang $\mathrm{Y}$, Schuff $\mathrm{N}$, Jahng $\mathrm{GH}$, et al. Diffusion tensor imaging of cingulum fibers in mild cognitive impairment and Alzheimer disease. Neurology 2007;68:1319.

147. Cho H, Yang DW, Shon YM, et al. Abnormal Integrity of Corticocortical Tracts in Mild Cognitive Impairment: A Diffusion Tensor Imaging Study. Journal of Korean Medical Science 2008;23:477-483.

148. Ding B, Chen KM, Ling HW, et al. Diffusion Tensor Imaging Correlates with Proton Magnetic Resonance Spectroscopy in Posterior Cingulate Region of Patients with Alzheimer,Äôs Disease. Dementia and Geriatric Cognitive Disorders 2008;25:218-225.

149. Fujie $\mathrm{S}$, Namiki C, Nishi $\mathrm{H}$, et al. The role of the uncinate fasciculus in memory and emotional recognition in amnestic mild cognitive impairment. Dementia and Geriatric Cognitive Disorders 2008;26:432-439.

150. Parente D, Gasparetto E, da Cruz LCH, et al. Potential Role of Diffusion Tensor MRI in the Differential Diagnosis of Mild Cognitive Impairment and Alzheimer's Disease. American Journal of Radiology 2008;190:1369-1374.

151. Shim YS, Yoon B, Shon Y-M, Ahn K-J, Yang D-W. Difference of the hippocampal and white matter microalterations in $\mathrm{MCl}$ patients according to the severity of subcortical vascular changes: Neuropsychological correlates of diffusion tensor imaging. Clinical Neurology and Neurosurgery 2008;110:552-561.

152. Ukmar M, Makuc E, Onor M, Garbin G, Trevisiol M, Cova M. Evaluation of white matter damage in patients with Alzheimer's disease and in patients with mild 
cognitive impairment by using diffusion tensor imaging. La Radiologia Medica 2008;113:915-922.

153. Yasmin H, Nakata Y, Aoki S, et al. Diffusion abnormalities of the uncinate fasciculus in Alzheimer's disease: diffusion tensor tract-specific analysis using a new method to measure the core of the tract. Neuroradiology 2008;50:293299.

154. Bai F, Zhang Z, Watson DR, et al. Abnormal integrity of association fiber tracts in amnestic mild cognitive impairment. Journal of the Neurological Sciences 2009;278:102-106.

155. Chen T-F, Chen Y-F, Cheng T-W, Hua M-S, Liu H-M, Chiu M-J. Executive dysfunction and periventricular diffusion tensor changes in amnesic mild cognitive impairment and early Alzheimer's disease. Human Brain Mapping 2009;30:3826-3836.

156. Kiuchi K, Morikawa M, Taoka T, et al. Abnormalities of the uncinate fasciculus and posterior cingulate fasciculus in mild cognitive impairment and early Alzheimer's disease: A diffusion tensor tractography study. Brain Research 2009;1287:184-191.

157. Liu Y, Spulber G, Lehtimäki KK, et al. Diffusion tensor imaging and Tract-Based Spatial Statistics in Alzheimer's disease and mild cognitive impairment. Neurobiology of Aging 2009; In Press, Corrected Proof.

158. Mielke MM, Kozauer NA, Chan KCG, et al. Regionally-specific diffusion tensor imaging in mild cognitive impairment and Alzheimer's disease. Neuroimage 2009;46:47-55.

159. Nakata $\mathrm{Y}$, Sato $\mathrm{N}$, Nemoto K, et al. Diffusion abnormality in the posterior cingulum and hippocampal volume: correlation with disease progression in Alzheimer's disease. Magnetic Resonance Imaging 2009;27:347-354.

160. Stricker NH, Schweinsburg BC, Delano-Wood L, et al. Decreased white matter integrity in late-myelinating fiber pathways in Alzheimer's disease supports retrogenesis. Neuroimage 2009;45:10-16.

161. Zarei M, Damoiseaux JS, Morgese C, et al. Regional White Matter Integrity Differentiates Between Vascular Dementia and Alzheimer Disease. Stroke 2009;40:773-779.

162. Catheline G, Periot O, Amirault M, et al. Distinctive alterations of the cingulum bundle during aging and Alzheimer's disease. Neurobiology of Aging 2010;31:1582-1592. 
163. Hong YJ, Yoon B, Shim YS, et al. Differences in Microstructural Alterations of the Hippocampus in Alzheimer Disease and Idiopathic Normal Pressure Hydrocephalus: A Diffusion Tensor Imaging Study. American Journal of Neuroradiology 2010;31:1867-1872.

164. Kantarci K, Avula R, Senjem ML, et al. Dementia with Lewy bodies and Alzheimer disease: neurodegenerative patterns characterized by DTI. Neurology 2010;74:1814-1821.

165. Pievani M, Agosta F, Pagani E, et al. Assessment of white matter tract damage in mild cognitive impairment and Alzheimer's disease. Human Brain Mapping 2010;31:1862-1875.

166. Pitel A-L, Chanraud S, Sullivan EV, Pfefferbaum A. Callosal microstructural abnormalities in Alzheimer's disease and alcoholism: same phenotype, different mechanisms. Psychiatry Research: Neuroimaging 2010;184:49-56.

167. Scola E, Bozzali M, Agosta F, et al. A diffusion tensor MRI study of patients with $\mathrm{MCl}$ and $A D$ with a 2-year clinical follow-up. Journal of Neurology, Neurosurgery and Psychiatry 2010;81:798-805.

168. Sjöbeck M, Elfgren C, Larsson E-M, et al. Alzheimer's disease (AD) and executive dysfunction. A case-control study on the significance of frontal white matter changes detected by diffusion tensor imaging (DTI). Archives of Gerontology and Geriatrics 2010;50:260-266.

169. Thillainadesan S, Wen W, Zhuang L, et al. Changes in Mild Cognitive Impairment and its subtypes as seen on Diffusion Tensor Imaging In Press.

170. Winblad B, Palmer K, Kivipelto $M$, et al. Mild cognitive impairment - beyond controversies, towards a consensus: report of the International Working Group on Mild Cognitive Impairment. Journal of Internal Medicine 2004;256:240246.

171. Mohs RC, Knopman D, Petersen RC, et al. Development of cognitive instruments for use in clinical trials of antidementia drugs : Additions to the Alzheimer's disease assessment scale that broaden its scope. Hagerstown, MD, ETATS-UNIS: Lippincott Williams \& Wilkins, 1997. 
Table 1. Search terms used to search the databases PUBMED and ISI WEB OF KNOWLEDGE

\section{Search terms}

("medial temporal lobe") and ("magnetic resonance imaging") and ("Alzheimer*" or "mild cognitive impairment")

("hippocampus") and ("magnetic resonance imaging") and ("Alzheimer*" or "mild cognitive impairment")

("entorhinal cortex") and ("magnetic resonance imaging") and ("Alzheimer*" or "mild cognitive impairment")

("parahippocampal gyrus") and ("magnetic resonance imaging") and ("Alzheimer*" or "mild cognitive impairment")

("diffusion tensor imaging") and ("Alzheimer*" or "mild cognitive impairment")

Table 2. Subject characteristics medial temporal lobe studies

\begin{tabular}{|c|c|c|c|c|c|c|}
\hline Study & $\mathrm{N}$ & Age & MMSE & CDR & Regions & $\begin{array}{l}\text { Diagnostic } \\
\text { criteria }\end{array}$ \\
\hline Pitkänen et al. (1996) [59] & & & & & $\mathrm{HC}$ & 1 \\
\hline Controls & 76 & $\begin{array}{l}59.7 \\
(19.9)\end{array}$ & & & & \\
\hline$A D$ & 55 & $\begin{array}{l}69.9 \\
(8.3)\end{array}$ & $\begin{array}{l}\text { No } \\
\text { data }\end{array}$ & No data & & \\
\hline Convit et al. (1997) [60] & & & & & $\mathrm{HC}$ & 5 \\
\hline Controls & 27 & $\begin{array}{l}69.3 \\
(8.3)\end{array}$ & $\begin{array}{l}29.3 \\
(5.5)\end{array}$ & & & \\
\hline $\mathrm{MCl}$ & 22 & $\begin{array}{l}74.1 \\
(7.3)\end{array}$ & $\begin{array}{l}28.3 \\
(5.4)\end{array}$ & & & \\
\hline$A D$ & 27 & $\begin{array}{l}72.3 \\
(7.4)\end{array}$ & $\begin{array}{l}18.3 \\
(4.3)\end{array}$ & No data & & \\
\hline Kaye et al. (1997) [61] & & & & & $\mathrm{HC}, \mathrm{PHG}$ & 1 \\
\hline Controls & 18 & $\begin{array}{l}86.8 \\
(1.9)\end{array}$ & 28.4 & & & \\
\hline$A D$ & 12 & $\begin{array}{l}90.4 \\
(5.2)\end{array}$ & 26.9 & No data & & \\
\hline Mori et al. (1997) [62] & & & & & $\mathrm{HC}, \mathrm{PHG}$ & 1 \\
\hline Controls & 12 & $\begin{array}{l}66.2 \\
(4.9)\end{array}$ & $\geq 28$ & & & \\
\hline$A D$ & 46 & $\begin{array}{l}70.3 \\
(7.1)\end{array}$ & $\begin{array}{l}19.6 \\
(3.5)\end{array}$ & No data & & \\
\hline Jack et al. (1998) [63] & & & & & $\mathrm{HC}$ & 1 \\
\hline
\end{tabular}




\begin{tabular}{|c|c|c|c|c|c|c|}
\hline Controls & 24 & $\begin{array}{l}81.04 \\
(3.78)\end{array}$ & $\begin{array}{l}28.79 \\
(1.28)\end{array}$ & & & \\
\hline$A D$ & 24 & $\begin{array}{l}80.42 \\
(4.02)\end{array}$ & $\begin{array}{l}20.74 \\
(4.60)\end{array}$ & $\begin{array}{l}0.85 \\
(0.43)\end{array}$ & & \\
\hline Juottonen et al. (1998) [64] & & & & & ERC & 2 \\
\hline Controls & 32 & $\begin{array}{l}72 \\
(4.1)\end{array}$ & $\begin{array}{l}28.3 \\
(1.4)\end{array}$ & & & \\
\hline$A D$ & 30 & $\begin{array}{l}70.3 \\
(8.5)\end{array}$ & $\begin{array}{l}20.7 \\
(3.7)\end{array}$ & $\begin{array}{l}1.53 \\
(0.51)\end{array}$ & & \\
\hline Krasuski et al. (1998) [65] & & & & & $\mathrm{HC}, \mathrm{PHG}$ & 1 \\
\hline Controls & 21 & $\begin{array}{l}69.3 \\
(6.8)\end{array}$ & $\begin{array}{l}29.7 \\
(0.2)\end{array}$ & & & \\
\hline$A D$ & 13 & $\begin{array}{l}71.2 \\
(8.3)\end{array}$ & $\begin{array}{l}23.7 \\
(2.7)\end{array}$ & No data & & \\
\hline Laakso et al. (1998) [66] & & & & & $\mathrm{HC}$ & 1 \\
\hline Controls & 42 & $\begin{array}{l}72.0 \\
(4.0)\end{array}$ & $\begin{array}{l}28.0 \\
(1.0)\end{array}$ & & & \\
\hline $\mathrm{MCl}$ & 42 & $\begin{array}{l}70.0 \\
(5.0)\end{array}$ & $\begin{array}{l}28.0 \\
(2.0)\end{array}$ & & & \\
\hline$A D$ & 55 & $\begin{array}{l}70.0 \\
(8.0)\end{array}$ & $\begin{array}{l}22.0 \\
(4.0)\end{array}$ & No data & & \\
\hline Visser et al. (1999) [8] & & & & & $\begin{array}{l}\text { HC, PHG, } \\
\text { MTA }\end{array}$ & 1 \\
\hline - Con & 18 & $\begin{array}{l}76.8 \\
(4.0)\end{array}$ & $\begin{array}{l}27.1 \\
(2.8)\end{array}$ & & & \\
\hline$-\quad A D$ & 7 & $\begin{array}{l}79.6 \\
(4.9) \\
\end{array}$ & $\begin{array}{l}16.6 \\
(6.0)\end{array}$ & No data & & \\
\hline Barber et al. (2000) [67] & & & & & $\mathrm{HC}, \mathrm{PHG}$ & 1 \\
\hline - Con & 26 & $\begin{array}{l}76.2 \\
(5.0)\end{array}$ & $\begin{array}{l}28.1 \\
(2.0)\end{array}$ & & & \\
\hline$-\quad A D$ & 22 & $\begin{array}{l}77.3 \\
(5.0)\end{array}$ & $\begin{array}{l}16.5 \\
(4.0)\end{array}$ & No data & & \\
\hline $\begin{array}{l}\text { de Toledo-Morrell et al. } \\
\text { (2000) [68] }\end{array}$ & & & & & $\mathrm{HC}, \mathrm{PHG}$ & 1 \\
\hline Controls & 30 & $\begin{array}{l}72.4 \\
(64-84)\end{array}$ & $\geq 28$ & & & \\
\hline$A D$ & 18 & $\begin{array}{l}68.6 \\
(49-84)\end{array}$ & $\begin{array}{l}24.1 \\
(20-28)\end{array}$ & No datta & & \\
\hline Laakso et al. (2000) [69] & & & & & $\mathrm{HC}, \mathrm{ERC}$ & 2 \\
\hline
\end{tabular}




\begin{tabular}{|c|c|c|c|c|c|c|}
\hline Con & 30 & $\begin{array}{l}69.0 \\
(9.0)\end{array}$ & $\begin{array}{l}29.0 \\
(1.0)\end{array}$ & & & \\
\hline$A D$ & 30 & $\begin{array}{l}73.0 \\
(9.0)\end{array}$ & $\begin{array}{l}20.0 \\
(4.0)\end{array}$ & $\begin{array}{l}1.27 \\
(0.83)\end{array}$ & & \\
\hline Xu et al. (2000) [70] & & & & & HC. ERC & 1 \\
\hline Controls & 30 & $\begin{array}{l}78.9 \\
(6.2)\end{array}$ & $\begin{array}{l}28.5 \\
(1.6)\end{array}$ & & & \\
\hline $\mathrm{MCl}$ & 30 & $\begin{array}{l}78.4 \\
(6.4)\end{array}$ & $\begin{array}{l}25.7 \\
(2.8)\end{array}$ & & & \\
\hline$A D$ & 30 & $\begin{array}{l}78.5 \\
(6.3)\end{array}$ & $\begin{array}{l}20.6 \\
(5.0)\end{array}$ & No data & & \\
\hline De Santi et al. (2001) [71] & & & & & $\mathrm{HC}$ & $1 ; 5$ \\
\hline Controls & 11 & $\begin{array}{l}76.5 \\
(5.4) \\
\end{array}$ & $\begin{array}{l}29.1 \\
(1.5)\end{array}$ & & & \\
\hline $\mathrm{MCl}$ & 15 & $\begin{array}{l}74.6 \\
(6.7)\end{array}$ & $\begin{array}{l}28.5 \\
(1.9)\end{array}$ & & & \\
\hline$A D$ & 12 & $\begin{array}{l}76.1 \\
(7.0) \\
\end{array}$ & $\begin{array}{l}20.3 \\
(7.2) \\
\end{array}$ & No data & & \\
\hline Du et al. (2001) [72] & & & & & $\mathrm{HC}, \mathrm{ERC}$ & 1 \\
\hline Controls & 40 & $\begin{array}{l}75.1 \\
(4.3)\end{array}$ & $\begin{array}{l}29.0 \\
(0.9)\end{array}$ & & & \\
\hline $\mathrm{MCl}$ & 36 & $\begin{array}{l}75.1 \\
(8.2) \\
\end{array}$ & $\begin{array}{l}25.8 \\
(3.6) \\
\end{array}$ & & & \\
\hline$A D$ & 29 & $\begin{array}{l}75.8 \\
(5.1) \\
\end{array}$ & $\begin{array}{l}17.7 \\
(5.7) \\
\end{array}$ & No data & & \\
\hline $\begin{array}{l}\text { Goncharova et al. (2001) } \\
\text { [73] }\end{array}$ & & & & & ERC & 1 \\
\hline Controls & 34 & $\begin{array}{l}70.3 \\
(6.6) \\
\end{array}$ & $\begin{array}{l}29.2 \\
(0.7) \\
\end{array}$ & & & \\
\hline$A D$ & 16 & $\begin{array}{l}71.4 \\
(9.1) \\
\end{array}$ & $\begin{array}{l}27.3 \\
(1.1) \\
\end{array}$ & No data & & \\
\hline Wolf et al. (2001) [74] & & & & & $\mathrm{HC}$ & 1 \\
\hline Controls & 17 & $\begin{array}{l}78.5 \\
(3.1) \\
\end{array}$ & $\begin{array}{l}28.3 \\
(1.3) \\
\end{array}$ & & & \\
\hline $\mathrm{MCl}$ & 12 & $\begin{array}{l}78.5 \\
(2.2) \\
\end{array}$ & $\begin{array}{l}25.7 \\
(1.1) \\
\end{array}$ & & & \\
\hline$A D$ & 10 & $\begin{array}{l}78.2 \\
(3.0)\end{array}$ & $\begin{array}{l}22.9 \\
(2.0) \\
\end{array}$ & No data & & \\
\hline Bottino et al. (2002) [75] & & & & & HC, PHG & 1 \\
\hline
\end{tabular}




\begin{tabular}{|c|c|c|c|c|c|c|}
\hline Controls & 20 & $\begin{array}{l}69.1 \\
(4.84)\end{array}$ & $\begin{array}{l}29.1 \\
(0.7)\end{array}$ & & & \\
\hline$A D$ & 39 & $\begin{array}{l}73.1 \\
(7.21)\end{array}$ & $\begin{array}{l}20.2 \\
(3.6)\end{array}$ & No data & & \\
\hline Hsu et al. (2002) [76] & & & & & $\mathrm{HC}$ & 1 \\
\hline Controls & 20 & $\begin{array}{l}74.0 \\
(6.2)\end{array}$ & $\begin{array}{l}29.0 \\
(1.3)\end{array}$ & & & \\
\hline $\mathrm{MCl}$ & 20 & $\begin{array}{l}74.2 \\
(6.7)\end{array}$ & $\begin{array}{l}27.7 \\
(2.7)\end{array}$ & & & \\
\hline$A D$ & 20 & $\begin{array}{l}74.5 \\
(6.2)\end{array}$ & $\begin{array}{l}22.7 \\
(3.5)\end{array}$ & No data & & \\
\hline Killiany et al. (2002) [77] & & & & & HC, ERC & 2 \\
\hline Controls & 28 & 71.8 & 29.2 & & & \\
\hline $\mathrm{MCl}$ & 94 & 72 & 29.1 & & & \\
\hline$A D$ & 16 & 69.8 & 24.2 & No data & & \\
\hline Mega et al. (2002) [78] & & & & & $\mathrm{HC}$ & 3 \\
\hline Controls & 10 & $\begin{array}{l}73.0 \\
(7.4)\end{array}$ & $\begin{array}{l}29.2 \\
(1.0)\end{array}$ & & & \\
\hline $\mathrm{MCl}$ & 10 & $\begin{array}{l}71.0 \\
(5.2)\end{array}$ & $\begin{array}{l}28.9 \\
(1.3)\end{array}$ & No data & & \\
\hline Pantel et al. (2002) [79] & & & & & HC, PHG & 1 \\
\hline Controls & 22 & $\begin{array}{l}65.3 \\
(0.8) \\
\end{array}$ & & & & \\
\hline $\mathrm{MCl}$ & 21 & $\begin{array}{l}65.2 \\
(0.9)\end{array}$ & & & & \\
\hline$A D$ & 12 & $\begin{array}{l}63.7 \\
(7.4)\end{array}$ & $\begin{array}{l}18.75 \\
(4.0)\end{array}$ & No data & & \\
\hline Du et al. (2003) [80] & & & & & ERC & 1 \\
\hline Controls & 23 & $\begin{array}{l}76.5 \\
(7.9) \\
\end{array}$ & $\begin{array}{l}29 \\
(1.0)\end{array}$ & & & \\
\hline$A D$ & 21 & $\begin{array}{l}74.5 \\
(6.7)\end{array}$ & $\begin{array}{l}22 \\
(7.0)\end{array}$ & No data & & \\
\hline Du et al. (2004) [81] & & & & & HC, ERC & 1 \\
\hline Controls & 25 & $\begin{array}{r}76.8 \\
(7.8) \\
\end{array}$ & $\begin{array}{l}29.0 \\
(1.0) \\
\end{array}$ & & & \\
\hline$A D$ & 20 & $\begin{array}{l}75.3 \\
(7.2)\end{array}$ & $\begin{array}{l}21.0 \\
(7.2)\end{array}$ & No data & & \\
\hline Pennanen et al. (2004) [37] & & & & & HC, ERC & $1 ; 5$ \\
\hline
\end{tabular}




\begin{tabular}{|c|c|c|c|c|c|c|}
\hline Controls & 59 & $\begin{array}{l}72.7 \\
(4.3)\end{array}$ & $\begin{array}{l}27.3 \\
(1.8)\end{array}$ & & & \\
\hline $\mathrm{MCl}$ & 65 & $\begin{array}{l}72.8 \\
(4.5)\end{array}$ & $\begin{array}{l}24.0 \\
(2.5)\end{array}$ & & & \\
\hline$A D$ & 48 & $\begin{array}{l}71.1 \\
(8.1)\end{array}$ & $\begin{array}{l}21.4 \\
(3.5)\end{array}$ & No data & & \\
\hline Testa et al. (2004) [82] & & & & & $\mathrm{HC}$ & 2 \\
\hline Controls & 25 & $\begin{array}{l}70.0 \\
(8.0)\end{array}$ & $\begin{array}{l}29.0 \\
(1.0)\end{array}$ & & & \\
\hline$A D$ & 27 & $\begin{array}{l}74.0 \\
(9.0)\end{array}$ & $\begin{array}{l}21.0 \\
(4.0) \\
\end{array}$ & No data & & \\
\hline Müller et al. (2005) [28] & & & & & $\mathrm{HC}$ & 3 \\
\hline Controls & 18 & $\begin{array}{l}66.9 \\
(9.0)\end{array}$ & $\begin{array}{l}28.7 \\
(1.0)\end{array}$ & & & \\
\hline $\mathrm{aMCl}$ & 18 & $\begin{array}{l}67.3 \\
(8.7)\end{array}$ & $\begin{array}{l}25.2 \\
(2.2)\end{array}$ & No data & & \\
\hline $\begin{array}{l}\text { Bastos-Leite et al. (2006) } \\
\text { [83] }\end{array}$ & & & & & $\begin{array}{l}\text { MTA- } \\
\text { score }\end{array}$ & 2 \\
\hline Controls & 15 & $\begin{array}{l}68.9 \\
(8.0)\end{array}$ & $\begin{array}{l}27.9 \\
(1.9)\end{array}$ & & & \\
\hline$A D$ & 21 & $\begin{array}{l}69.3 \\
(10.9)\end{array}$ & $\begin{array}{l}18.8 \\
(4.5)\end{array}$ & No data & & \\
\hline Kalus et al. (2006) [84] & & & & & $\begin{array}{l}\text { HC, ERC, } \\
\text { PHG }\end{array}$ & 1 \\
\hline Controls & 10 & $\begin{array}{l}70.8 \\
(5.6)\end{array}$ & $\begin{array}{l}29.0 \\
(27-30)\end{array}$ & & & \\
\hline $\mathrm{MCl}$ & 10 & $\begin{array}{l}76.0 \\
(11.5)\end{array}$ & $\begin{array}{l}22.4 \\
(19-28)\end{array}$ & & & \\
\hline$A D$ & 10 & $\begin{array}{l}73.9 \\
(9.8)\end{array}$ & $\begin{array}{l}19.2 \\
(6-27)\end{array}$ & No data & & \\
\hline Teipel et al. (2006) [85] & & & & & $\begin{array}{l}\mathrm{HC}, \mathrm{ERC}, \\
\mathrm{PHG}\end{array}$ & 2 \\
\hline Controls & 22 & $\begin{array}{l}61.5 \\
(8.9)\end{array}$ & $\begin{array}{l}29.4 \\
(0.7)\end{array}$ & & & \\
\hline$A D$ & 34 & $\begin{array}{l}69.0 \\
(8.0)\end{array}$ & $\begin{array}{l}23.1 \\
(4.1)\end{array}$ & No data & & \\
\hline Uotani et al. (2006) [86] & & & & & $\mathrm{HC}$ & 5 \\
\hline Controls & 20 & $\begin{array}{l}65.7 \\
(8.7)\end{array}$ & $\begin{array}{l}29.6 \\
(0.8)\end{array}$ & & & \\
\hline
\end{tabular}




\begin{tabular}{|c|c|c|c|c|c|c|}
\hline $\mathrm{MCl}$ & 7 & $\begin{array}{l}73.2 \\
(8.6)\end{array}$ & $\begin{array}{l}26.9 \\
(2.1)\end{array}$ & & & \\
\hline$A D$ & 33 & $\begin{array}{l}70.9 \\
(8.3)\end{array}$ & $\begin{array}{l}14.1 \\
(8.1)\end{array}$ & No data & & \\
\hline van de Pol et al. (2006) [87] & & & & & $\begin{array}{l}\text { HC, MTA- } \\
\text { score }\end{array}$ & 1 \\
\hline Controls & 73 & $\begin{array}{l}75.0 \\
(8.0)\end{array}$ & $\begin{array}{l}\text { No } \\
\text { data }\end{array}$ & & & \\
\hline$A D$ & 103 & $\begin{array}{l}71.0 \\
(9.0)\end{array}$ & $\begin{array}{l}\text { No } \\
\text { data }\end{array}$ & $\begin{array}{l}1.0(0.5- \\
3)\end{array}$ & & \\
\hline Barnes et al. (2007) [88] & & & & & $\mathrm{HC}$ & 7 \\
\hline Controls & 11 & $\begin{array}{l}56.0 \\
(14.3)\end{array}$ & $29(1)$ & & & \\
\hline$A D$ & 19 & $\begin{array}{l}56.3 \\
(10.6)\end{array}$ & $20(7)$ & No data & & \\
\hline Meyer et al. (2007) [89] & & & & & HC, ERC & 1,4 \\
\hline Controls & 52 & $\begin{array}{l}65.6 \\
(11.0)\end{array}$ & $\begin{array}{l}29.50 \\
(0.73)\end{array}$ & & & \\
\hline $\mathrm{MCl}$ & 30 & $\begin{array}{l}77.2 \\
(8.52)\end{array}$ & $\begin{array}{l}26.27 \\
(1.80)\end{array}$ & & & \\
\hline$A D$ & 19 & $\begin{array}{l}78.74 \\
(5.05)\end{array}$ & $\begin{array}{l}16.84 \\
(9.66)\end{array}$ & No data & & \\
\hline Ridha et al. (2007) [90] & & & & & $\begin{array}{l}\text { HC, MTA- } \\
\text { score }\end{array}$ & 2 \\
\hline Controls & 47 & $\begin{array}{l}65.5 \\
(11.4) \\
\end{array}$ & $\begin{array}{l}29.5 \\
(0.7) \\
\end{array}$ & & & \\
\hline$A D$ & 26 & $\begin{array}{l}65.7 \\
(11.5)\end{array}$ & $\begin{array}{l}19.4 \\
(4.0)\end{array}$ & No data & & \\
\hline Slavin et al. (2007) [91] & & & & & $\mathrm{HC}$ & $1 ; 5$ \\
\hline Controls & 17 & $\begin{array}{l}70.2 \\
(3.6)\end{array}$ & $\begin{array}{l}28.2 \\
(1.3)\end{array}$ & & & \\
\hline $\mathrm{aMCl}$ & 18 & $\begin{array}{l}74.4 \\
(7.7) \\
\end{array}$ & $\begin{array}{l}26.8 \\
(1.3)\end{array}$ & No data & & \\
\hline Colliot et al. (2008) [92] & & & & & $\mathrm{HC}$ & $1 ; 3$ \\
\hline Controls & 25 & $\begin{array}{l}64.0 \\
(8.0) \\
\end{array}$ & $\begin{array}{l}\text { No } \\
\text { data }\end{array}$ & & & \\
\hline $\mathrm{aMCl}$ & 24 & $\begin{array}{l}74.0 \\
(8.0)\end{array}$ & $\begin{array}{l}27.2 \\
(1.4)\end{array}$ & & & \\
\hline
\end{tabular}




\begin{tabular}{|c|c|c|c|c|c|c|}
\hline$A D$ & 25 & $\begin{array}{l}73.0 \\
(6.0)\end{array}$ & $\begin{array}{l}24.4 \\
(2.7)\end{array}$ & No data & & \\
\hline $\begin{array}{l}\text { Delano-Wood et al. (2008) } \\
\text { [93] }\end{array}$ & & & & & $\mathrm{HC}$ & 3 \\
\hline Controls & 20 & $\begin{array}{l}78.14 \\
(7.06) \\
\end{array}$ & & & & \\
\hline $\mathrm{MCl}$ & 20 & $\begin{array}{l}78.00 \\
(7.32)\end{array}$ & $\begin{array}{l}\text { No } \\
\text { data }\end{array}$ & No data & & \\
\hline Duara et al. (2008) [94] & & & & & $\begin{array}{l}\text { MTA- } \\
\text { score }\end{array}$ & $1 ; 3$ \\
\hline Controls & 117 & $\begin{array}{l}71.7 \\
(5.7)\end{array}$ & $\begin{array}{l}29 \\
(1.2)\end{array}$ & & & \\
\hline $\mathrm{aMCl}$ & 91 & $\begin{array}{l}76.4 \\
(6.0) \\
\end{array}$ & $\begin{array}{l}26.6 \\
(2.3) \\
\end{array}$ & & & \\
\hline$A D$ & 53 & $\begin{array}{l}79.9 \\
(6.0)\end{array}$ & $\begin{array}{l}22.9 \\
(3.8)\end{array}$ & No data & & \\
\hline Kenny et al. (2008) [95] & & & & & ERC & 1 \\
\hline Controls & 37 & $\begin{array}{r}75.4 \\
(6.8) \\
\end{array}$ & $\begin{array}{l}28.1 \\
(1.6) \\
\end{array}$ & & & \\
\hline$A D$ & 26 & $\begin{array}{l}78.1 \\
(5.0) \\
\end{array}$ & $\begin{array}{l}18.7 \\
(4.4) \\
\end{array}$ & No data & & \\
\hline Appel et al. (2009) [96] & & & & & $\begin{array}{l}\text { MTA- } \\
\text { score }\end{array}$ & $1 ; 3$ \\
\hline Controls & 40 & $\begin{array}{l}71.0 \\
(5.6) \\
\end{array}$ & $\begin{array}{l}29.3 \\
(0.9) \\
\end{array}$ & & & \\
\hline (a) $\mathrm{MCl}$ & 118 & $\begin{array}{l}74.7 \\
(6.3) \\
\end{array}$ & $\begin{array}{l}26.9 \\
(2.3) \\
\end{array}$ & & & \\
\hline$A D$ & 34 & $\begin{array}{l}76.6 \\
(6.5) \\
\end{array}$ & $\begin{array}{l}22.7 \\
(2.8) \\
\end{array}$ & No data & & \\
\hline Bai et al. (2009) [97] & & & & & $\mathrm{HC}$ & 3 \\
\hline Controls & 23 & $\begin{array}{l}70 \\
(5.0)\end{array}$ & $\begin{array}{l}28.3 \\
(1.5) \\
\end{array}$ & & & \\
\hline $\mathrm{aMCl}$ & 39 & $\begin{array}{l}71 \\
(5.0) \\
\end{array}$ & $\begin{array}{l}27 \\
(1.6) \\
\end{array}$ & No data & & \\
\hline Cho et al. (2009) [98] & & & & & $\begin{array}{l}\text { MTA- } \\
\text { score }\end{array}$ & 1 \\
\hline Controls & 27 & $\begin{array}{l}\text { No } \\
\text { data }\end{array}$ & $\begin{array}{l}\text { No } \\
\text { data }\end{array}$ & & & \\
\hline
\end{tabular}




\begin{tabular}{|c|c|c|c|c|c|c|}
\hline$A D$ & 33 & $\begin{array}{l}74.4 \\
(7.8) \\
\end{array}$ & $\begin{array}{l}16.55 \\
(4.40) \\
\end{array}$ & $1.7(0.81)$ & & \\
\hline Feczko et al. (2009) [99] & & & & & ERC & 1 \\
\hline Controls & 47 & $\begin{array}{l}76.2 \\
(6.8) \\
\end{array}$ & $\begin{array}{l}29.0 \\
(1.3) \\
\end{array}$ & 0 & & \\
\hline$A D$ & 29 & $\begin{array}{l}73.8 \\
(11.7)\end{array}$ & $\begin{array}{l}22.9 \\
(3.8)\end{array}$ & $\begin{array}{l}0.88 \\
(0.22) \\
\end{array}$ & & \\
\hline Ferrarini et al. (2009) [100] & & & & & $\mathrm{HC}$ & 1 \\
\hline Controls & 50 & $\begin{array}{l}70.8 \\
(5.8) \\
\end{array}$ & $\begin{array}{l}28.1 \\
(1.4) \\
\end{array}$ & & & \\
\hline $\mathrm{MCl}$ & 30 & $\begin{array}{l}71.9 \\
(6.2) \\
\end{array}$ & $\begin{array}{l}26.7 \\
(1.3) \\
\end{array}$ & & & \\
\hline$A D$ & 50 & $\begin{array}{l}71.3 \\
(7.7) \\
\end{array}$ & $\begin{array}{l}18.5 \\
(3.4)\end{array}$ & No data & & \\
\hline $\begin{array}{l}\text { Henneman et al. (2009) } \\
\text { [101] }\end{array}$ & & & & & $\begin{array}{l}\text { MTA- } \\
\text { score }\end{array}$ & $2 ; 3$ \\
\hline Controls & 19 & $\begin{array}{l}66 \\
(9.0) \\
\end{array}$ & $\begin{array}{l}29 \\
(1.0)\end{array}$ & & & \\
\hline $\mathrm{MCl}$ & 25 & $\begin{array}{l}71 \\
(6.0) \\
\end{array}$ & $\begin{array}{l}25 \\
(3.0) \\
\end{array}$ & & & \\
\hline$A D$ & 31 & $\begin{array}{l}67 \\
(8.0)\end{array}$ & $\begin{array}{l}23 \\
(4.0)\end{array}$ & No data & & \\
\hline $\begin{array}{l}\text { Henneman et al. (2009) } \\
\text { [102] }\end{array}$ & & & & & $\begin{array}{l}\text { HC, MTA- } \\
\text { score }\end{array}$ & $2 ; 3$ \\
\hline Controls & 34 & $\begin{array}{l}67 \\
(9.0) \\
\end{array}$ & $\begin{array}{l}28 \\
(2.0) \\
\end{array}$ & & & \\
\hline $\mathrm{MCl}$ & 44 & $\begin{array}{l}71 \\
(6.0) \\
\end{array}$ & $\begin{array}{l}26 \\
(3.0) \\
\end{array}$ & & & \\
\hline$A D$ & 64 & $\begin{array}{l}67 \\
(9.0) \\
\end{array}$ & $\begin{array}{l}22 \\
(5.0) \\
\end{array}$ & No data & & \\
\hline Hyun et al. (2009) [103] & & & & & $\begin{array}{l}\text { MTA- } \\
\text { score }\end{array}$ & 1 \\
\hline Controls & 27 & $\begin{array}{l}\text { No } \\
\text { data }\end{array}$ & $\begin{array}{l}\text { No } \\
\text { data }\end{array}$ & & & \\
\hline$A D$ & 33 & $\begin{array}{l}74.4 \\
(7.8) \\
\end{array}$ & $\begin{array}{l}16.55 \\
(4.40) \\
\end{array}$ & $1.7(0.81)$ & & \\
\hline $\begin{array}{l}\text { Jauhiainen et al. (2009) } \\
\text { [104] }\end{array}$ & & & & & HC, ERC & $2 ; 3$ \\
\hline
\end{tabular}




\begin{tabular}{|c|c|c|c|c|c|c|}
\hline Controls & 21 & $\begin{array}{l}71.2 \\
(4.9)\end{array}$ & $\begin{array}{l}27.7 \\
(2.0)\end{array}$ & & & \\
\hline aMCl & 14 & $\begin{array}{l}72.4 \\
(7.3)\end{array}$ & $\begin{array}{l}25.6 \\
(3.1)\end{array}$ & & & \\
\hline$A D$ & 15 & $\begin{array}{l}73.1 \\
(6.7)\end{array}$ & $\begin{array}{l}21.7 \\
(3.7)\end{array}$ & $0.8(0.3)$ & & \\
\hline Lee et al. (2009) [105] & & & & & $\mathrm{HC}$ & $2 ; 4$ \\
\hline Controls & 95 & $\begin{array}{l}72.7 \\
(7.1)\end{array}$ & $\begin{array}{l}29.7 \\
(10.5)\end{array}$ & & & \\
\hline $\mathrm{MCl}$ & 73 & $\begin{array}{l}73.6 \\
(7.5)\end{array}$ & $\begin{array}{l}27.2 \\
(12.9)\end{array}$ & & & \\
\hline$A D$ & 47 & $\begin{array}{l}77.2 \\
(7.2)\end{array}$ & $\begin{array}{l}21.2 \\
(12.9)\end{array}$ & No data & & \\
\hline $\begin{array}{l}\text { Loewenstein et al. (2009) } \\
\text { [106] }\end{array}$ & & & & & MTA & 6 \\
\hline Controls & 108 & $\begin{array}{l}72.23 \\
(5.4)\end{array}$ & & & & \\
\hline $\mathrm{aMCl}$ & 78 & $\begin{array}{l}76.27 \\
(6.4)\end{array}$ & & & & \\
\hline$A D$ & 48 & $\begin{array}{l}77.08 \\
(6.2)\end{array}$ & $\begin{array}{l}\text { No } \\
\text { data }\end{array}$ & No data & & \\
\hline Morra et al. (2009) [107] & & & & & $\mathrm{HC}$ & $1 ; 3$ \\
\hline Controls & 100 & $\begin{array}{l}76.6 \\
(4.8)\end{array}$ & $\begin{array}{l}29.1 \\
(0.9)\end{array}$ & $0.0(0.9)$ & & \\
\hline $\mathrm{MCl}$ & 200 & $\begin{array}{l}75.4 \\
(7.0)\end{array}$ & $\begin{array}{l}26.9 \\
(1.9)\end{array}$ & $1.5(0.8)$ & & \\
\hline$A D$ & 100 & $\begin{array}{l}75.9 \\
(7.2)\end{array}$ & $\begin{array}{l}23.4 \\
(1.9)\end{array}$ & $4.5(1.6)$ & & \\
\hline Rogalski et al. (2009) [108] & & & & & $\begin{array}{l}\mathrm{HC}, \mathrm{ERC}, \\
\mathrm{PHG}\end{array}$ & 3 \\
\hline Controls & 14 & $\begin{array}{l}73.6 \\
(6.7) \\
\end{array}$ & $\begin{array}{l}29.4 \\
(0.8)\end{array}$ & & & \\
\hline $\mathrm{aMCl}$ & 14 & $\begin{array}{l}76.8 \\
(7.0)\end{array}$ & $\begin{array}{l}26.9 \\
(2.0)\end{array}$ & No data & & \\
\hline Bird et al. (2010) [109] & & & & & MTA & 1 \\
\hline Controls & 25 & $\begin{array}{l}65.3 \\
(7.6)\end{array}$ & & & & \\
\hline $\mathrm{aMCl}$ & 6 & $\begin{array}{l}65.3 \\
(11.0)\end{array}$ & $\begin{array}{l}27.0 \\
(1.5)\end{array}$ & & & \\
\hline
\end{tabular}




\begin{tabular}{|c|c|c|c|c|c|c|}
\hline$A D$ & 7 & $\begin{array}{l}66.6 \\
(7.5)\end{array}$ & $\begin{array}{l}26.1 \\
(2.8)\end{array}$ & No data & & \\
\hline $\begin{array}{l}\text { Bouwman et al. (2010) } \\
\text { [110] }\end{array}$ & & & & & MTA & 1 \\
\hline Controls & 138 & $\begin{array}{l}60.0 \\
(10.0)\end{array}$ & $\begin{array}{l}28.3 \\
(1.8)\end{array}$ & & & \\
\hline $\mathrm{MCl}$ & 65 & $\begin{array}{l}70.0 \\
(8.0)\end{array}$ & $\begin{array}{l}26.3 \\
(2.7)\end{array}$ & & & \\
\hline$A D$ & 145 & $\begin{array}{l}68.8 \\
(8.0) \\
\end{array}$ & $\begin{array}{l}21.5 \\
(4.6)\end{array}$ & No data & & \\
\hline Cherubini et al. (2010) [111] & & & & & $\mathrm{HC}$ & $2 ; 3$ \\
\hline Controls & 30 & $\begin{array}{l}67.9 \\
(7.4)\end{array}$ & $\begin{array}{l}28.97 \\
(1.24)\end{array}$ & & & \\
\hline $\mathrm{aMCl}$ & 30 & $\begin{array}{l}66.8 \\
(6.0)\end{array}$ & $\begin{array}{l}27.57 \\
(2.10)\end{array}$ & & & \\
\hline$A D$ & 30 & $\begin{array}{l}68.7 \\
(7.8)\end{array}$ & $\begin{array}{l}21.33 \\
(4.03) \\
\end{array}$ & No data & & \\
\hline Choo et al. (2010) [33] & & & & & $\mathrm{HC}, \mathrm{ERC}$ & $2 ; 5$ \\
\hline Controls & 18 & $\begin{array}{l}70.7 \\
(5.2) \\
\end{array}$ & $\begin{array}{l}\text { No } \\
\text { data }\end{array}$ & & & \\
\hline $\mathrm{MCl}$ & 19 & $\begin{array}{l}71.6 \\
(7.1)\end{array}$ & & & & \\
\hline$A D$ & 19 & $\begin{array}{l}71.1 \\
(5.1)\end{array}$ & & $\begin{array}{l}0.95 \\
(0.44)\end{array}$ & & \\
\hline Desikan et al. (2010) [112] & & & & & $\mathrm{HC}$ & $1 ; 3$ \\
\hline Controls & 208 & $\begin{array}{l}76.0 \\
(4.9) \\
\end{array}$ & $\begin{array}{l}29.1 \\
(1.0)\end{array}$ & 0 & & \\
\hline $\mathrm{MCl}$ & 353 & $\begin{array}{l}74.5 \\
(7.4)\end{array}$ & $\begin{array}{l}27.0 \\
(1.8)\end{array}$ & $1.6(0.9)$ & & \\
\hline$A D$ & 163 & $\begin{array}{l}74.9 \\
(7.5) \\
\end{array}$ & $\begin{array}{l}23.3 \\
(1.9) \\
\end{array}$ & $4.2(1.6)$ & & \\
\hline Jhoo et al. (2010) [113] & & & & & $\mathrm{HC}$ & $2 ; 4 ; 5$ \\
\hline Controls & 17 & $\begin{array}{l}70.8 \\
(5.4) \\
\end{array}$ & $\begin{array}{l}28.4 \\
(1.9) \\
\end{array}$ & & & \\
\hline $\mathrm{aMCl}$ & 17 & $\begin{array}{l}70.8 \\
(7.0) \\
\end{array}$ & $\begin{array}{l}24.4 \\
(3.7) \\
\end{array}$ & & & \\
\hline$A D$ & 17 & $\begin{array}{l}70.7 \\
(5.8) \\
\end{array}$ & $\begin{array}{l}16.9 \\
(3.8)\end{array}$ & $\begin{array}{l}0.79 \\
(0.25) \\
\end{array}$ & & \\
\hline Johnson et al. (2010) [114] & & & & & $\mathrm{HC}$ & 1 \\
\hline
\end{tabular}




\begin{tabular}{|c|c|c|c|c|c|c|}
\hline Controls & 32 & $\begin{array}{l}70.3 \\
(6.3)\end{array}$ & $\begin{array}{l}29.4 \\
(0.84)\end{array}$ & & & \\
\hline$A D$ & 27 & $\begin{array}{l}73.0 \\
(6.5)\end{array}$ & $\begin{array}{l}27.5 \\
(2.25)\end{array}$ & 0.5 & & \\
\hline Lehmann et al. (2010) [115] & & & & & $\begin{array}{l}\mathrm{HC}, \mathrm{ERC} \text {, } \\
\mathrm{PHG}\end{array}$ & 2 \\
\hline Controls & 10 & $\begin{array}{l}59.7 \\
(6.3)\end{array}$ & $\begin{array}{l}29.8 \\
(0.4)\end{array}$ & & & \\
\hline$A D$ & 10 & $\begin{array}{l}60.0 \\
(7.6)\end{array}$ & $\begin{array}{l}20.4 \\
(5.8)\end{array}$ & No data & & \\
\hline Liu et al. (2010) [116] & & & & & $\mathrm{HC}$ & $2 ; 3$ \\
\hline Controls & 94 & $\begin{array}{l}74.0 \\
(5.0)\end{array}$ & $\begin{array}{l}29.0 \\
(1.0)\end{array}$ & & & \\
\hline $\mathrm{MCl}$ & 79 & $\begin{array}{l}74.0 \\
(6.0)\end{array}$ & $\begin{array}{l}27.0 \\
(2.0)\end{array}$ & & & \\
\hline$A D$ & 118 & $\begin{array}{l}75.0 \\
(6.0)\end{array}$ & $\begin{array}{l}21.0 \\
(5.0)\end{array}$ & $1.3(0.5)$ & & \\
\hline Luckhaus et al. (2010) [117] & & & & & $\mathrm{HC}$ & 2 \\
\hline Controls & 12 & $\begin{array}{l}65.7 \\
(4.1) \\
\end{array}$ & $\begin{array}{l}28.0 \\
(1.53) \\
\end{array}$ & & & \\
\hline $\mathrm{MCl}$ & 30 & $\begin{array}{l}64.1 \\
(9.5)\end{array}$ & $\begin{array}{l}27.5 \\
(1.88)\end{array}$ & No data & & \\
\hline$A D$ & 15 & $\begin{array}{l}68.7 \\
(7.6) \\
\end{array}$ & $\begin{array}{l}23.47 \\
(1.88) \\
\end{array}$ & & & \\
\hline Mueller et al. (2010) [118] & & & & & HC, ERC & $1 ; 3$ \\
\hline Controls & 53 & $\begin{array}{l}69.5 \\
(7.3) \\
\end{array}$ & $\begin{array}{l}29.3 \\
(1.1) \\
\end{array}$ & & & \\
\hline $\mathrm{aMCl}$ & 20 & $\begin{array}{l}73.5 \\
(7.1) \\
\end{array}$ & $\begin{array}{l}28.0 \\
(2.1)\end{array}$ & & & \\
\hline$A D$ & 18 & $\begin{array}{l}69.1 \\
(9.6) \\
\end{array}$ & $\begin{array}{l}21.6 \\
(5.1) \\
\end{array}$ & No data & & \\
\hline Pengas et al. (2010) [119] & & & & & $\mathrm{HC}$ & 2 \\
\hline Controls & 28 & $\begin{array}{l}64.9 \\
(61-73) \\
\end{array}$ & $\begin{array}{l}29.3 \\
(0.8) \\
\end{array}$ & & & \\
\hline $\mathrm{aMCl}$ & 24 & $\begin{array}{l}67.6 \\
(63-72) \\
\end{array}$ & $\begin{array}{l}26.8 \\
(1.4)\end{array}$ & No data & & \\
\hline Ryu et al. (2010) [120] & & & & & $\mathrm{HC}$ & 2 \\
\hline Controls & 14 & $\begin{array}{l}70.6 \\
(5.2)\end{array}$ & $\begin{array}{l}24.1 \\
(2.3)\end{array}$ & & & \\
\hline
\end{tabular}




\begin{tabular}{|c|c|c|c|c|c|c|}
\hline$A D$ & 23 & $\begin{array}{l}74.4 \\
(6.3)\end{array}$ & $\begin{array}{l}17.6 \\
(4.8)\end{array}$ & No data & & \\
\hline $\begin{array}{l}\text { Sanchez-Benavides et al. } \\
\text { (2010) [121] }\end{array}$ & & & & & HC, ERC & $1 ; 5$ \\
\hline Controls & 34 & $\begin{array}{l}71.7 \\
(5.9)\end{array}$ & $\begin{array}{l}28.9 \\
(1.2)\end{array}$ & & & \\
\hline $\mathrm{MCl}$ & 24 & $\begin{array}{l}74.3 \\
(6.2)\end{array}$ & $\begin{array}{l}26.0 \\
(2.3)\end{array}$ & No data & & \\
\hline$A D$ & 20 & $\begin{array}{l}75.3 \\
(6.6)\end{array}$ & $\begin{array}{l}21.4 \\
(3.4)\end{array}$ & & & \\
\hline Schott et al. (2010) [122] & & & & & $\mathrm{HC}$ & $1 ; 3$ \\
\hline Controls & 199 & $\begin{array}{l}76.0 \\
(5.1)\end{array}$ & $\begin{array}{l}29.1 \\
(1.0)\end{array}$ & $\begin{array}{l}0.03 \\
(0.11)\end{array}$ & & \\
\hline $\mathrm{MCl}$ & 334 & $\begin{array}{l}74.9 \\
(7.2)\end{array}$ & $\begin{array}{l}27.0 \\
(1.8)\end{array}$ & $\begin{array}{l}1.58 \\
(0.89)\end{array}$ & & \\
\hline$A D$ & 144 & $\begin{array}{l}75.2 \\
(7.3)\end{array}$ & $\begin{array}{l}23.5 \\
(1.9)\end{array}$ & $\begin{array}{l}4.19 \\
(1.55)\end{array}$ & & \\
\hline Westman et al. (2010) [123] & & & & & $\mathrm{HC}$ & $1 ; 5$ \\
\hline Controls & 100 & $\begin{array}{l}73.0 \\
(6.0) \\
\end{array}$ & $\begin{array}{l}29.0 \\
(1.0) \\
\end{array}$ & 0 & & \\
\hline $\mathrm{MCl}$ & 100 & $\begin{array}{l}75.0 \\
(5.0)\end{array}$ & $\begin{array}{l}27.0 \\
(3.0)\end{array}$ & 0.5 & & \\
\hline$A D$ & 100 & $\begin{array}{l}76.0 \\
(6.0)\end{array}$ & $\begin{array}{l}21.0 \\
(5.0)\end{array}$ & $1.2(0.5)$ & & \\
\hline Yakushev et al. (2010) [124] & & & & & $\mathrm{HC}$ & 1 \\
\hline Controls & 18 & $\begin{array}{l}69.0 \\
(6.7) \\
\end{array}$ & $\begin{array}{l}28.9 \\
(1.0) \\
\end{array}$ & & & \\
\hline$A D$ & 20 & $\begin{array}{l}69.8 \\
(7.4) \\
\end{array}$ & $\begin{array}{l}25.7 \\
(1.7)\end{array}$ & $\begin{array}{l}0.63 \\
(0.22)\end{array}$ & & \\
\hline Zarei et al. (2010) [125] & & & & & $\mathrm{HC}$ & 2 \\
\hline Controls & 22 & $\begin{array}{l}70.7 \\
(6.0) \\
\end{array}$ & $\begin{array}{l}28.7 \\
(1.4) \\
\end{array}$ & & & \\
\hline$A D$ & 16 & $\begin{array}{l}69.5 \\
(6.7)\end{array}$ & $\begin{array}{l}22.9 \\
(3.2)\end{array}$ & No data & & \\
\hline Echavarri et al. (2011) [126] & & & & & $\mathrm{HC}, \mathrm{PHG}$ & $1 ; 3$ \\
\hline Controls & 18 & $\begin{array}{l}64.5 \\
(3.3)\end{array}$ & $\begin{array}{l}28.8 \\
(27-30)\end{array}$ & & & \\
\hline $\mathrm{MCl}$ & 18 & $\begin{array}{l}65.1 \\
(4.5)\end{array}$ & $\begin{array}{l}27.6 \\
(22-30)\end{array}$ & & & \\
\hline
\end{tabular}




\begin{tabular}{|l|l|l|l|l|l|l|}
\hline AD & 18 & $\begin{array}{l}72.2 \\
(9.7)\end{array}$ & $\begin{array}{l}21(10- \\
28)\end{array}$ & No data & & \\
\hline Jacobs et al. (2011) [44] & & & & & HC, PHG & 5 \\
\hline Controls & 35 & $\begin{array}{l}69.1 \\
(7.7)\end{array}$ & $\begin{array}{l}28.2 \\
(1.5)\end{array}$ & & & \\
\hline $\mathrm{MCl}$ & 30 & $\begin{array}{l}69.2 \\
(8.1)\end{array}$ & $\begin{array}{l}26.3 \\
(2.0)\end{array}$ & & & \\
\hline AD & 9 & $\begin{array}{l}73.8 \\
(4.3)\end{array}$ & $\begin{array}{l}24.8 \\
(3.1)\end{array}$ & No data & & \\
\hline Prestia et al. (2011) [127] & & & & & HC & 1 \\
\hline Controls & 19 & $\begin{array}{l}72.5 \\
(7.8)\end{array}$ & $\begin{array}{l}29.1 \\
(1.0)\end{array}$ & & & \\
\hline AD & 20 & 72.7 & 22.0 & No data & & \\
& & $(9.1)$ & $(4.3)$ & & & \\
\hline Zhang et al. (2011) [128] & & & & & HC & 3 \\
\hline Controls & 243 & 77.8 & & & & \\
\hline MCl & 146 & 78.8 & No & No data & & \\
\hline & & & & & \\
\hline
\end{tabular}

Data are mean (sd) or mean (range).Volumetric measurements of HC: hippocampus, ERC: entorhinal cortex, PHG: parahippocampal gyrus.MMSE: Mini-mental state examination; CDR: Clinical Dementia rating scale (AD group); MTA: Medial temporal lobe atrophy; Con: healthy controls; $\mathrm{MCl}$ : mild cognitive impairment, subtypes not specified or all subtypes included; aMCl: amnestic-type $\mathrm{MCl}$; AD: Alzheimer's disease. Main diagnostic criteria: (1) NINCDS-ADRDA: probable and/or possible AD = McKhann et al., 1984 [129]; (2) NINCDS-ADRDA: probable AD - McKhann et al., 1984 [129]; (3) MCI according to Petersen et al., (1999) [3] or (2001) [130]; (4) MCl according to Winblad et al., 2004; (5) Diagnostic and Statistical Manual of Mental Disorders (DSM) Fourth edition; (6) National Alzheimer Coordinating Center guidelines (NACC) - Washington.; (7) Based on genetic or pathological analysis. 


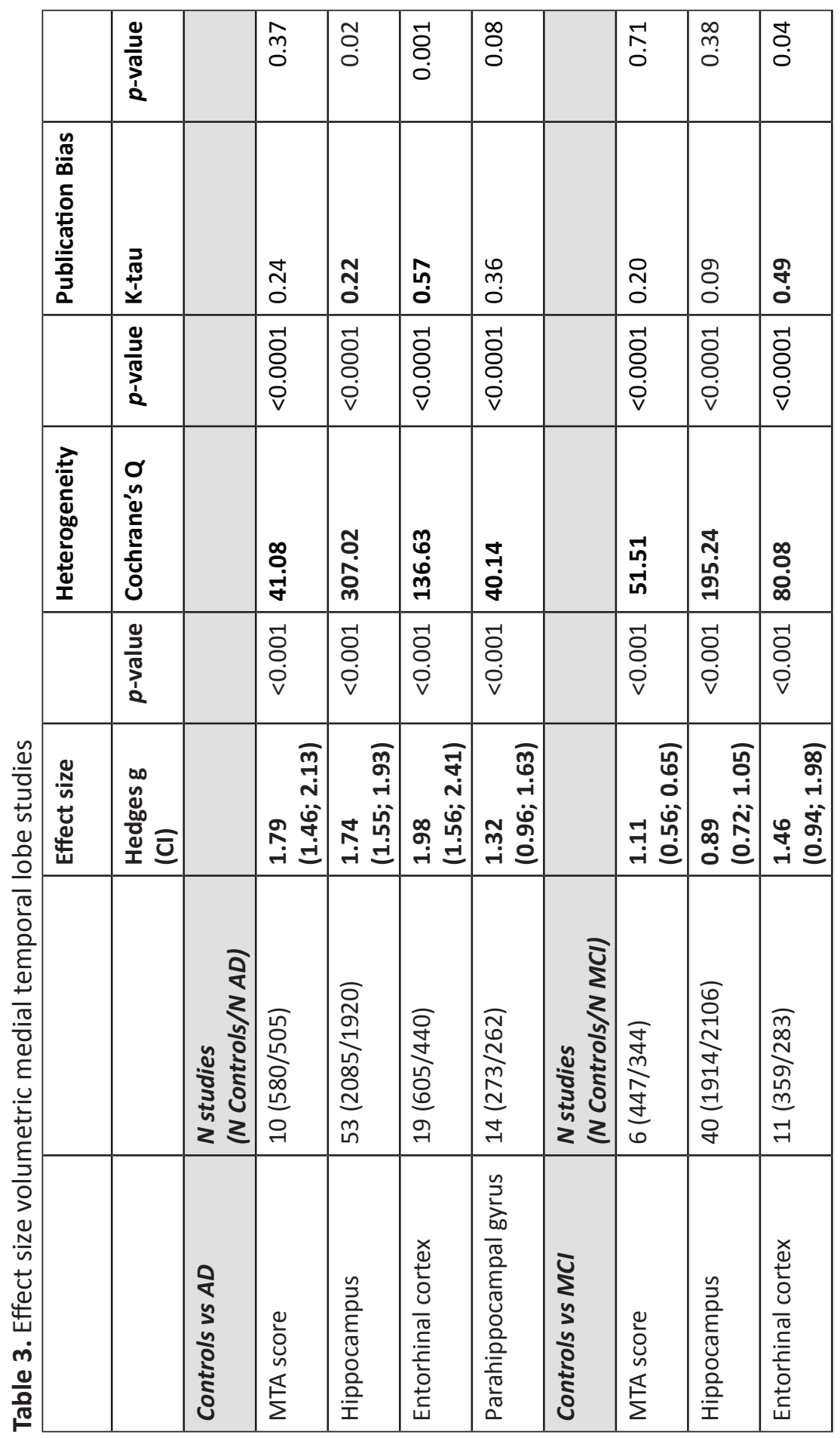

168 | Chapter 5: Meta-analysis DTI and MTL measurements 


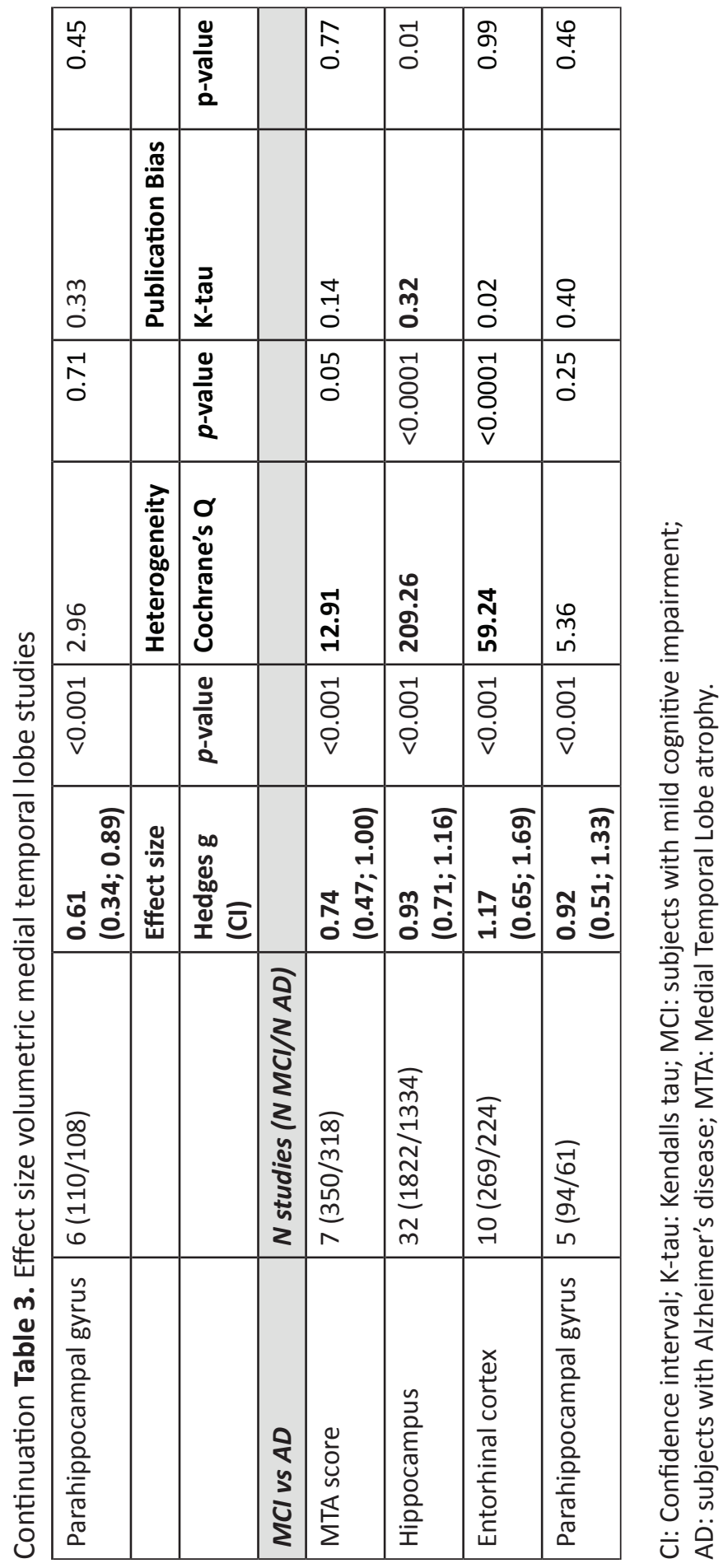

Chapter 5: Meta-analysis DTI and MTL measurements | 169 


\begin{tabular}{|c|c|c|c|c|c|c|c|c|c|c|c|c|}
\hline & $\underline{\underline{u}}$ & $\times$ & $x$ & $x$ & & & & & & $x$ & $x$ & \\
\hline & $\underline{x}$ & & & & $x$ & & & & & & & \\
\hline & 꼼 & & & & & & & & & & & \\
\hline & ज & $\times$ & & & & & & & & & & \\
\hline & Ј & & & & & & & & & & & \\
\hline & 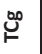 & $\times$ & & & & & & & & & & \\
\hline & $\frac{\text { mon }}{2}$ & & & & & & & & & & & \\
\hline & ס̊ & & & & & & & & $x$ & & & $x$ \\
\hline & $\sum^{\infty}$ & & & & & & & & & & & \\
\hline & 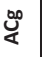 & & & & & & & & & & & \\
\hline & U్ & $\times$ & & $x$ & $x$ & $x$ & $x$ & $\times$ & & $x$ & $\times$ & \\
\hline & U్ & & & $x$ & $x$ & $x$ & $x$ & $\times$ & & $x$ & $x$ & \\
\hline & $\vec{F}$ & & $x$ & $x$ & $x$ & $x$ & & $x$ & & $x$ & $x$ & \\
\hline 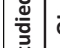 & ठ & & $x$ & $\times$ & $x$ & $x$ & & & & $x$ & $x$ & \\
\hline $\begin{array}{ll}n \\
\underline{n} \\
\vdots\end{array}$ & $\vec{a}$ & & $x$ & $\times$ & $x$ & $x$ & & & & $x$ & $x$ & \\
\hline & $\vec{u}$ & & $\times$ & $x$ & $\times$ & $\times$ & & $x$ & & $x$ & $x$ & \\
\hline & $\stackrel{\square}{\circ}$ & 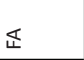 & 足匹 & $\llbracket$ & 足匹 & 足匹 & $\overleftarrow{4}$ & 足匹 & 号匹 & 号芯 & 号匹 & $\overleftrightarrow{4}$ \\
\hline & ப̆ & -1 & n & $\sim$ & $\begin{array}{l}m \\
\dot{i} \\
\end{array}$ & $\rightarrow$ & $\begin{array}{l}0 \\
\end{array}$ & $\begin{array}{l}0 \\
\stackrel{-}{*} \\
-1\end{array}$ & $\begin{array}{l}m \\
\dot{i} \\
\end{array}$ & N & $\sim$ & $\because \ddot{m}$ \\
\hline & ů & $\begin{array}{l}\frac{8}{0} \\
\frac{\pi}{8} \\
0 \\
2 \\
\end{array}$ & $\begin{array}{l}\frac{\pi}{0} \\
\frac{\pi}{0} \\
0 \\
2 \\
\end{array}$ & $\begin{array}{l}\mathbb{0} \\
\frac{\pi}{0} \\
0 \\
0 \\
2\end{array}$ & $\begin{array}{l}\frac{5}{5} \\
\frac{\pi}{10} \\
0 \\
2 \\
\end{array}$ & $\begin{array}{l}\sqrt{n} \\
0 \\
\\
0 \\
\end{array}$ & $\begin{array}{l}\frac{\pi}{0} \\
\frac{\pi}{10} \\
0 \\
2\end{array}$ & $\begin{array}{l}\frac{\pi}{70} \\
\frac{\pi}{0} \\
2 \\
2 \\
\end{array}$ & $\begin{array}{l}\frac{\pi}{0} \\
\frac{\pi}{0} \\
0 \\
2 \\
\end{array}$ & $\begin{array}{l}\frac{\pi}{0} \\
\frac{\pi}{0} \\
\frac{0}{2} \\
2\end{array}$ & $\begin{array}{l}\frac{\pi}{10} \\
\frac{10}{0} \\
0 \\
2 \\
\end{array}$ & $\begin{array}{l}\frac{\pi}{0} \\
\frac{\pi}{80} \\
2 \\
2\end{array}$ \\
\hline & $\sum_{\Sigma}^{u}$ & 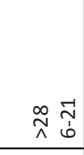 & 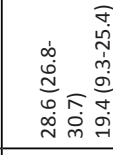 & 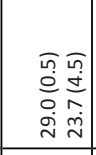 & 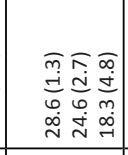 & 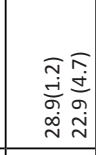 & $\begin{array}{l}\frac{\pi}{10} \\
\frac{\pi}{0} \\
2 \\
2\end{array}$ & 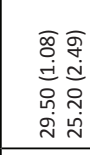 & 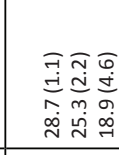 & 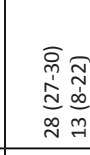 & 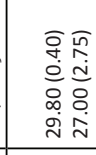 & 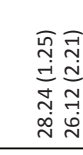 \\
\hline & 总 & 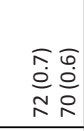 & 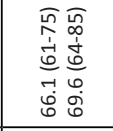 & 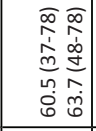 & 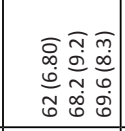 & $\begin{array}{l}\sqrt{n} \frac{\Phi}{R} \\
\therefore\end{array}$ & 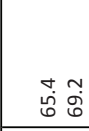 & 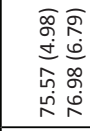 & 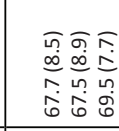 & 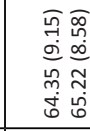 & 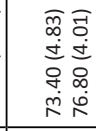 & 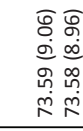 \\
\hline & $z$ & の 7 & 우국 & 요 & 욱 ${ }_{f}$ 의 & $\stackrel{\sim}{\sim}$ & 우 요 & 억으 & $\vec{\sim} \approx \stackrel{\Perp}{\sim}$ & $\approx \stackrel{0}{\sim}$ & $\approx \approx$ & 구 \\
\hline & $\begin{array}{l}\text { 咅 } \\
\text { w }\end{array}$ & 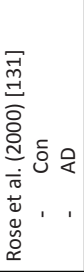 & 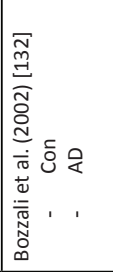 & 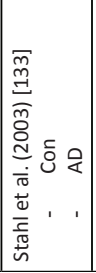 & 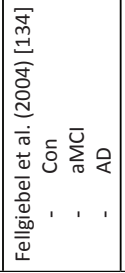 & 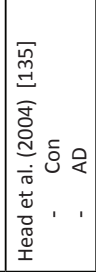 & 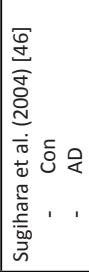 & 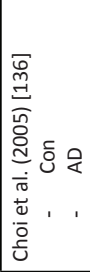 & 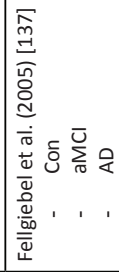 & 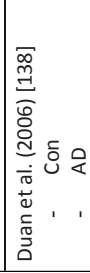 & 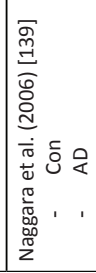 & 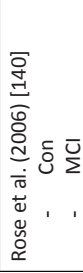 \\
\hline
\end{tabular}




\begin{tabular}{|c|c|c|c|c|c|c|c|c|c|c|c|}
\hline & $\underline{\underline{u}}$ & & & $x$ & & & $x$ & $x$ & $x$ & & \\
\hline & uㅗ & & $x$ & & & $x$ & & & $x$ & & \\
\hline & 꼼 & & & & & & & $x$ & & & \\
\hline & 岕 & & & & & & & & $x$ & & \\
\hline & 岂 & $x$ & & & & & & & & & \\
\hline & 吅 & & & & & & & & & & \\
\hline & $\frac{\text { mo }}{a}$ & & & & & & & & & & \\
\hline & ֻ̊ & & $\times$ & & & & & $x$ & $x$ & & $\times$ \\
\hline & $\stackrel{\infty}{\Sigma}^{\infty}$ & & $\times$ & & & & & & & & \\
\hline & 苂 & & & & & & & & & & \\
\hline & U్ & & & & & & $\times$ & $x$ & $x$ & $\times$ & \\
\hline & U্ & & & & & & $x$ & $x$ & $x$ & $x$ & \\
\hline & $F$ & & & $x$ & $\times$ & & $x$ & $x$ & $x$ & & \\
\hline 㕺 & ठ & & & $x$ & $\times$ & & $x$ & & $x$ & & \\
\hline $\begin{array}{l}n \\
\underline{n} \\
\underline{0}\end{array}$ & $\vec{a}$ & & & $x$ & $\times$ & & $x$ & & $x$ & & \\
\hline 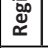 & $\vec{\mu}$ & & & $x$ & $\times$ & & $x$ & & $x$ & & \\
\hline & $\begin{array}{l}\bar{Z} \\
\Sigma \\
\Sigma\end{array}$ & 选 & 足匹 & 这 & 뚜 & 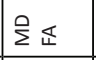 & 近 & 这 & 这 & 足芯 & 足匹 \\
\hline & 口 & 0 & N & $\sim$ & $\stackrel{m}{m}$ & $m$ & $\begin{array}{l}m \\
i \\
i\end{array}$ & $\dot{\sim} \hat{m}$ & $m$ & $m$ & $N$ \\
\hline & ơ & $\begin{array}{l}\frac{7}{7} \\
\frac{\pi}{10} \\
0 \\
2 \\
\end{array}$ & $\begin{array}{l}\frac{5}{0} \\
\frac{\pi}{8} \\
0 \\
2 \\
\end{array}$ & $\begin{array}{l}\frac{9}{10} \\
\frac{10}{0} \\
2 \\
2\end{array}$ & $\begin{array}{l}\frac{\pi}{0} \\
\text { 苂 } \\
0 \\
2 \\
\end{array}$ & $\begin{array}{l}\frac{\pi}{0} \\
\frac{\pi}{8} \\
0 \\
2 \\
\end{array}$ & $\begin{array}{l}\frac{\pi}{0} \\
\frac{\pi}{0} \\
0 \\
2 \\
\end{array}$ & 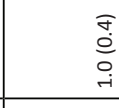 & $\begin{array}{l}\frac{\pi}{0} \\
\frac{\pi}{8} \\
0 \\
2 \\
\end{array}$ & $\begin{array}{l}\frac{\pi}{0} \\
\frac{\pi}{0} \\
0 \\
2 \\
\end{array}$ & 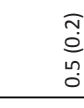 \\
\hline & $\sum_{\Sigma}^{\underline{\omega}}$ & 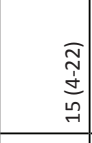 & $\begin{array}{l}\frac{9}{0} \\
\frac{\pi}{0} \\
0 \\
2 \\
\end{array}$ & 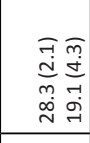 & 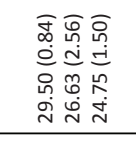 & 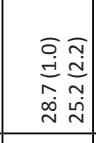 & 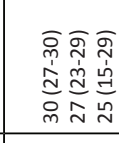 & 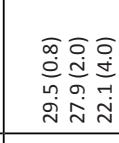 & 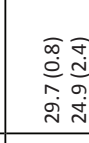 & $\begin{array}{l}\frac{\pi}{0} \\
\frac{\pi}{0} \\
0 \\
2 \\
\end{array}$ & 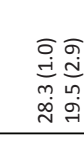 \\
\hline & $\begin{array}{l}\text { : } \\
\stackrel{4}{<} \\
\end{array}$ & 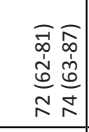 & 욧 & $\begin{array}{l}0 \\
0 \\
\end{array}$ & 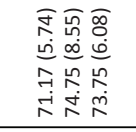 & 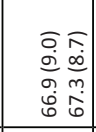 & 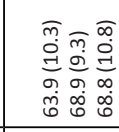 & 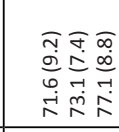 & 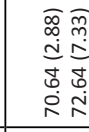 & 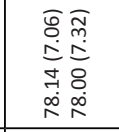 & 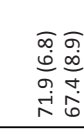 \\
\hline & $z$ & 녹 ص & 어요 이 & 녹 워 & $\sigma \infty \sigma$ & $\stackrel{\infty}{\infty} \underset{-}{-\infty}$ & 의 & $\stackrel{\infty}{\sim}$ ન & $\exists \exists$ & 오 & 오 \\
\hline & 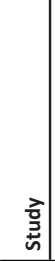 & 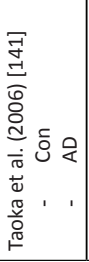 & 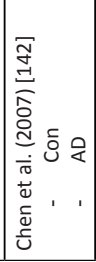 & 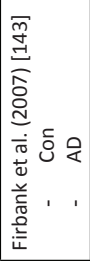 & 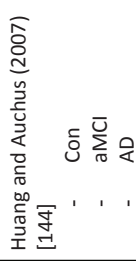 & 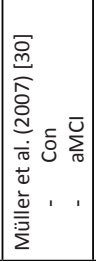 & 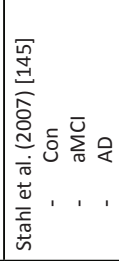 & 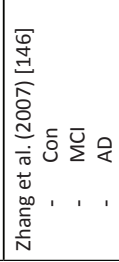 & 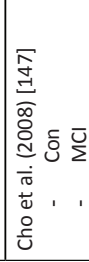 & 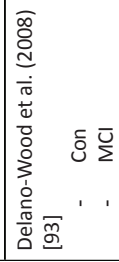 & 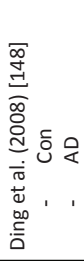 \\
\hline
\end{tabular}




\begin{tabular}{|c|c|c|c|c|c|c|c|c|c|c|c|}
\hline & $\underline{\cup}$ & & & & & & & & & & \\
\hline & $\underline{\underline{x}}$ & & $x$ & $x$ & & & & & & & \\
\hline & 옴 & & & & & & & & & & \\
\hline & 岕 & & $\times$ & & & & $x$ & & & & \\
\hline & 岂 & $x$ & & & & $x$ & & & & $x$ & \\
\hline & 吅 & & & & & & & & & & \\
\hline & 꼼 & & & & & & & & & & \\
\hline & هొ & & $x$ & & & & $x$ & & & $x$ & \\
\hline & $\tilde{\Sigma}^{\infty}$ & & & & & & & & & & \\
\hline & 幽 & & & & & & & & & & \\
\hline & ü & & $x$ & $x$ & $\times$ & & $x$ & $\times$ & $x$ & & $x$ \\
\hline & Ŭ & & & $x$ & $x$ & & $x$ & $\times$ & $x$ & & $\times$ \\
\hline & $F$ & & & $x$ & $x$ & & & $x$ & $x$ & & \\
\hline 䓀 & ठ & & & $\times$ & $\times$ & & & & & & $x$ \\
\hline $\begin{array}{l}n \\
\underline{n} \\
\underline{0}\end{array}$ & $\vec{a}$ & & & $x$ & $x$ & & & & & & $\times$ \\
\hline 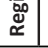 & $\vec{\mu}$ & & & $x$ & $x$ & & & & & & $x$ \\
\hline & $\begin{array}{l}\text { D. } \\
\Sigma \\
\end{array}$ & $\overleftarrow{4}$ & 匹 & 这 & $\llbracket$ & 足匹 & 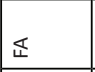 & 这 & 这匹 & $\sum_{\Sigma}^{\mathbb{L}}$ & 芒 \\
\hline & ષ̆ & $m$ & 6 & in & $\begin{array}{l}\ddot{0} \\
\dot{m} \\
\dot{m} \overline{\bar{\varepsilon}}\end{array}$ & n & $m$ & $\begin{array}{c}m \\
\dot{i} \\
\end{array}$ & $\begin{array}{l}m \\
\dot{i} \\
\end{array}$ & $\begin{array}{l}\infty \\
\dot{N} \\
\end{array}$ & $\stackrel{n}{i}$ \\
\hline & ố & $\begin{array}{l}\frac{8}{0} \\
\frac{\pi}{8} \\
0 \\
2 \\
\end{array}$ & $\begin{array}{l}\frac{\pi}{10} \\
\frac{\pi}{0} \\
0 \\
2 \\
\end{array}$ & $\begin{array}{l}\frac{5}{0} \\
\frac{\pi}{0} \\
0 \\
2 \\
\end{array}$ & $\begin{array}{l}\frac{\pi}{0} \\
\frac{\pi}{0} \\
0 \\
2 \\
\end{array}$ & $\begin{array}{l}\frac{\pi}{\pi} \\
\frac{\pi}{0} \\
0 \\
2\end{array}$ & $\begin{array}{l}\frac{\pi}{0} \\
\frac{\pi}{0} \\
0 \\
2 \\
\end{array}$ & $\begin{array}{l}\frac{8}{5} \\
\frac{\pi}{0} \\
2 \\
\end{array}$ & 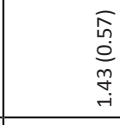 & $\begin{array}{l}\frac{9}{0} \\
\frac{\pi}{10} \\
0 \\
2 \\
\end{array}$ & $\begin{array}{l}\frac{\pi}{0} \\
\frac{\pi}{0} \\
\frac{0}{2} \\
\end{array}$ \\
\hline & $\sum_{\Sigma}^{u}$ & 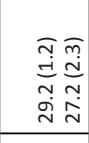 & 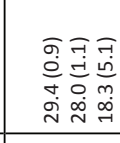 & 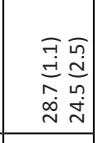 & 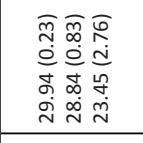 & 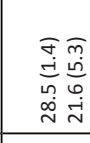 & 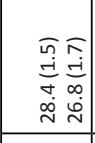 & $\begin{array}{l}\frac{5}{5} \\
\frac{\pi}{0} \\
0 \\
2 \\
\end{array}$ & $\begin{array}{l}\frac{\pi}{10} \\
\frac{10}{0} \\
2 \\
\end{array}$ & 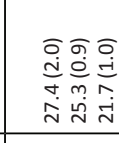 & 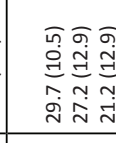 \\
\hline & \begin{tabular}{l} 
\\
$\square$ \\
\hdashline
\end{tabular} & 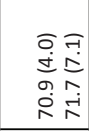 & 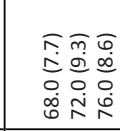 & 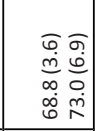 & 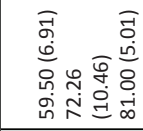 & 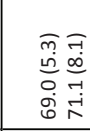 & 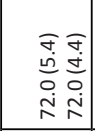 & 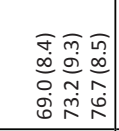 & 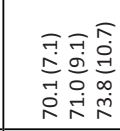 & 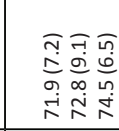 & 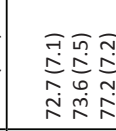 \\
\hline & $z$ & $\stackrel{9}{\varphi}$ & $\stackrel{\sim}{\sim} \stackrel{\sim}{\sim}$ & $\bar{A} \vec{N}$ & $\stackrel{\infty}{\sim} \stackrel{\sim}{\sim}$ & 욱 & $\approx \approx$ & $\stackrel{r}{\rightarrow} \underset{\sim}{-1}$ & 옹 ㅇ & $\stackrel{\sim}{0} \underset{-1}{-1}$ & ถักร \\
\hline & 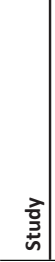 & 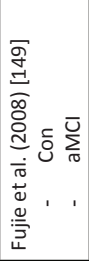 & 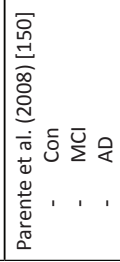 & 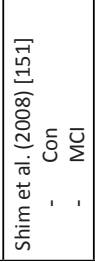 & 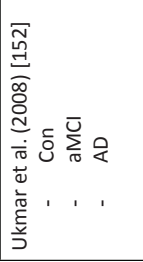 & 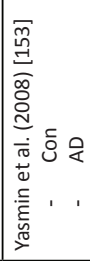 & 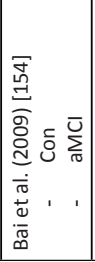 & 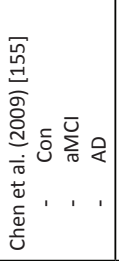 & 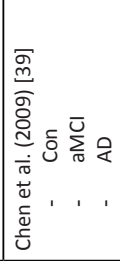 & 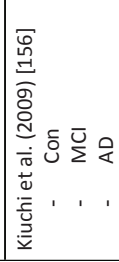 & 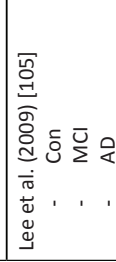 \\
\hline
\end{tabular}




\begin{tabular}{|c|c|c|c|c|c|c|c|c|c|c|c|}
\hline & $\underline{\underline{u}}$ & & & & & $\times$ & & & & & \\
\hline & $\underline{x}$ & & & & & & & & & & $\times$ \\
\hline & 옴 & & & & $x$ & & & & & & \\
\hline & 岕 & $\times$ & & & & $\times$ & & & & & \\
\hline & 岂 & & & & & & & & $x$ & & \\
\hline & ֻٌ & & & & & & & & & & \\
\hline & $\frac{\text { on }}{a}$ & & $\times$ & & & & & & $\times$ & $\times$ & \\
\hline & "̊ & & $x$ & $x$ & & & & & $\times$ & $\times$ & \\
\hline & $\stackrel{n}{\Sigma}^{\infty}$ & & & & & & & & & $\times$ & \\
\hline & 这 & & $\times$ & & & & & & $\times$ & $\times$ & \\
\hline & U్ & $\times$ & $x$ & & & & $x$ & & $\times$ & & \\
\hline & Ŭ & $\times$ & & & & & $\times$ & & $\times$ & & \\
\hline & $\vec{F}$ & $\times$ & & & & & $x$ & $\times$ & & & \\
\hline 㕺 & ठ & & & & & & & $\times$ & & & \\
\hline $\begin{array}{l}n \\
\tilde{c} \\
\vdots\end{array}$ & $\vec{a}$ & & & & & & & & & & \\
\hline 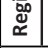 & $\vec{\mu}$ & & & & & & $\times$ & & & & \\
\hline & $\begin{array}{l}\overline{0} \\
\Sigma\end{array}$ & $\overleftarrow{4}$ & 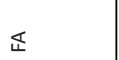 & 号匹 & 足匹 & $\overleftarrow{\Psi}$ & $\sum \overleftarrow{\Sigma}$ & $\overleftarrow{\Psi}$ & $\mathbb{4}$ & $\mathbb{4}$ & $\Sigma$ \\
\hline & : & $\stackrel{m}{i}$ & $\begin{array}{l}\infty \\
\dot{i} \\
\end{array}$ & N & $m$ & 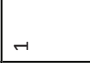 & $m$ & N & -1 & - & $\stackrel{m}{i}$ \\
\hline & ố & 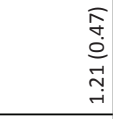 & 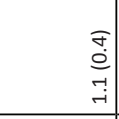 & $\begin{array}{l}\frac{\pi}{2} \\
\text { so } \\
0 \\
2 \\
\end{array}$ & $\begin{array}{l}\frac{\pi}{0} \\
\frac{\pi}{0} \\
0 \\
2 \\
\end{array}$ & $\begin{array}{l}\frac{\pi}{0} \\
\text { 贾 } \\
0 \\
2 \\
\end{array}$ & $\begin{array}{l}\frac{\pi}{0} \\
\frac{\pi}{0} \\
0 \\
2 \\
\end{array}$ & $\begin{array}{l}\bar{y} \\
0 \\
. \\
-1 \\
0 \\
0\end{array}$ & $\begin{array}{l}\frac{\pi}{0} \\
\frac{\pi}{0} \\
0 \\
2\end{array}$ & $\begin{array}{l}\frac{9}{0} \\
\frac{\pi}{10} \\
0 \\
2 \\
\end{array}$ & $\begin{array}{l}\frac{5}{0} \\
\frac{\pi}{0} \\
0 \\
2\end{array}$ \\
\hline & $\sum_{\Sigma}^{\underline{\omega}}$ & 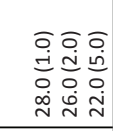 & 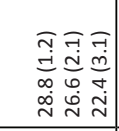 & 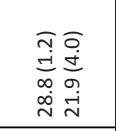 & 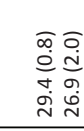 & 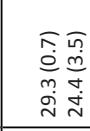 & 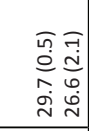 & 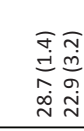 & 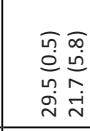 & 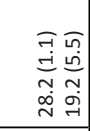 & 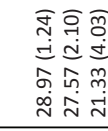 \\
\hline & 总 & 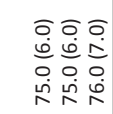 & 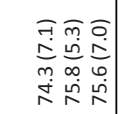 & 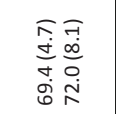 & 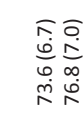 & 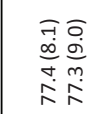 & 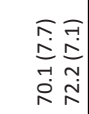 & 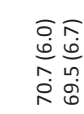 & 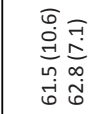 & 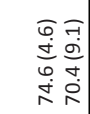 & 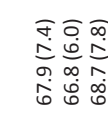 \\
\hline & $z$ & ㅊำ & $\stackrel{\sim}{\sim} \stackrel{n}{\sim}$ & $\stackrel{\infty}{\sim} \sim$ & ন & $\underset{\neg}{\neg}$ & 융ㅇ & $\approx \stackrel{1}{\circ}$ & g) & 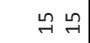 & 앺요 \\
\hline & 를 & 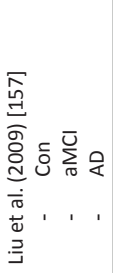 & 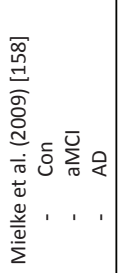 & 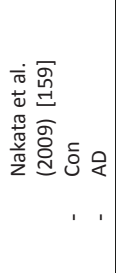 & 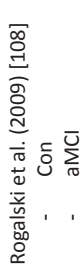 & 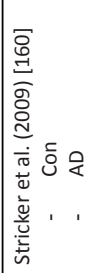 & 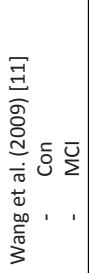 & 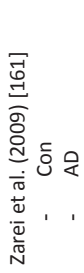 & 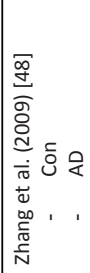 & 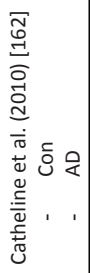 & 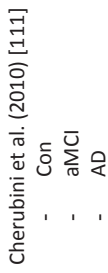 \\
\hline
\end{tabular}




\begin{tabular}{|c|c|c|c|c|c|c|c|c|c|c|}
\hline & $\underline{u}$ & & & $\times$ & & & & & & \\
\hline & ب̃ & $x$ & & & & & & $\times$ & & \\
\hline & 꼼 & & & $\times$ & & & & & & \\
\hline & 岕 & & & $x$ & $\times$ & $x$ & & & & \\
\hline & 岇 & & & $\times$ & & $\times$ & & & & \\
\hline & $\stackrel{\circ}{\stackrel{n}{\llcorner}}$ & & & $x$ & & & & & $\times$ & \\
\hline & 㕸 & & $\times$ & & & & & & $\times$ & \\
\hline & סֵّ & & & & $\times$ & & & $\times$ & & \\
\hline & $\sum_{\Sigma}^{\infty}$ & & & & & & & & & \\
\hline & סֶ & & & & & & & $x$ & & \\
\hline & ŭ & & & $\times$ & & & $\times$ & $\times$ & & \\
\hline & પ્ & & & $\times$ & & & $x$ & $x$ & & \\
\hline & $\vec{F}$ & & & & & & & $x$ & & \\
\hline 岁 & ठ & & & & & & & $\times$ & & $\times$ \\
\hline $\begin{array}{l}\bar{n} \\
\underline{c} \\
\bar{c}\end{array}$ & $\vec{a}$ & & & $\times$ & & & & $x$ & & \\
\hline 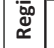 & $\vec{u}$ & & & $x$ & & & & $x$ & & $\times$ \\
\hline & $\stackrel{\text { Do }}{\Sigma}$ & $\varangle \frac{1}{\Sigma}$ & 氐 & $\llbracket \frac{0}{\Sigma}$ & $\llbracket \frac{0}{\Sigma}$ & 足匹 & $\llbracket \frac{0}{\Sigma}$ & ¿ष & 足出 & $\overleftarrow{\longleftarrow}$ \\
\hline & ப̆ & n & $\dot{\sim} \stackrel{0}{\sim}$ & -1 & - & $\stackrel{m}{\rightarrow}$ & n & n & $\stackrel{0}{-i}$ & N \\
\hline & 啥 & $\begin{array}{l}\infty \\
0 \\
0 \\
0 \\
\hat{0} \\
0 \\
\end{array}$ & 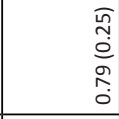 & $\stackrel{\text { ก }}{\text { o }}$ & 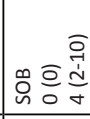 & $\begin{array}{l}\frac{\pi}{2} \\
\frac{\pi}{0} \\
0 \\
2 \\
\end{array}$ & $\begin{array}{l}\frac{9}{5} \\
\frac{\pi}{\pi} \\
0 \\
2 \\
\end{array}$ & $\begin{array}{l}\text { 焉 } \\
\frac{10}{10} \\
2 \\
2 \\
\end{array}$ & \begin{tabular}{l|} 
\\
$\frac{9}{10}$ \\
$\frac{10}{10}$ \\
2 \\
2
\end{tabular} & $\begin{array}{l}\frac{9}{0} \\
\frac{10}{0} \\
0 \\
2\end{array}$ \\
\hline & $\sum_{\Sigma}^{u}$ & 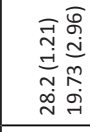 & 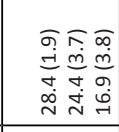 & 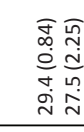 & $\begin{array}{l}\frac{\pi}{0} \\
\frac{\pi}{8} \\
0 \\
2\end{array}$ & 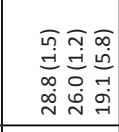 & 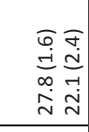 & 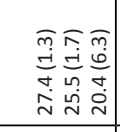 & 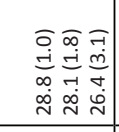 & 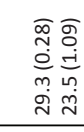 \\
\hline & 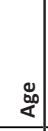 & 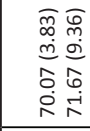 & 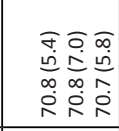 & 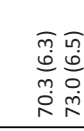 & 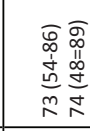 & 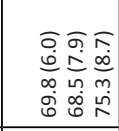 & 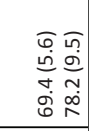 & 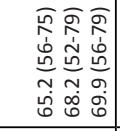 & 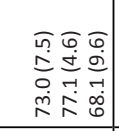 & 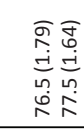 \\
\hline & $z$ & 늑 의 & 촉 & $\tilde{m} \approx$ & 8 i & $\stackrel{\sim}{\sim} \underset{\sim}{\mathcal{\sim}} \stackrel{\sim}{\sim}$ & $\tilde{m} \rightarrow$ & 우 & $\infty \infty \wedge$ & 늠 \\
\hline & 竧 & 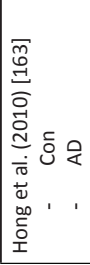 & 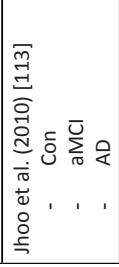 & 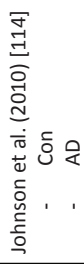 & 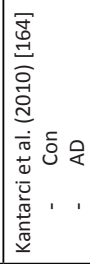 & 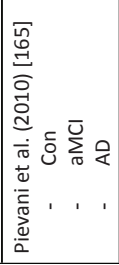 & 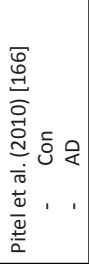 & 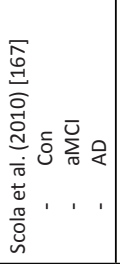 & 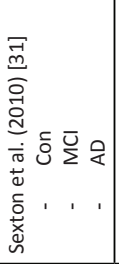 & 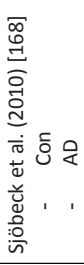 \\
\hline
\end{tabular}




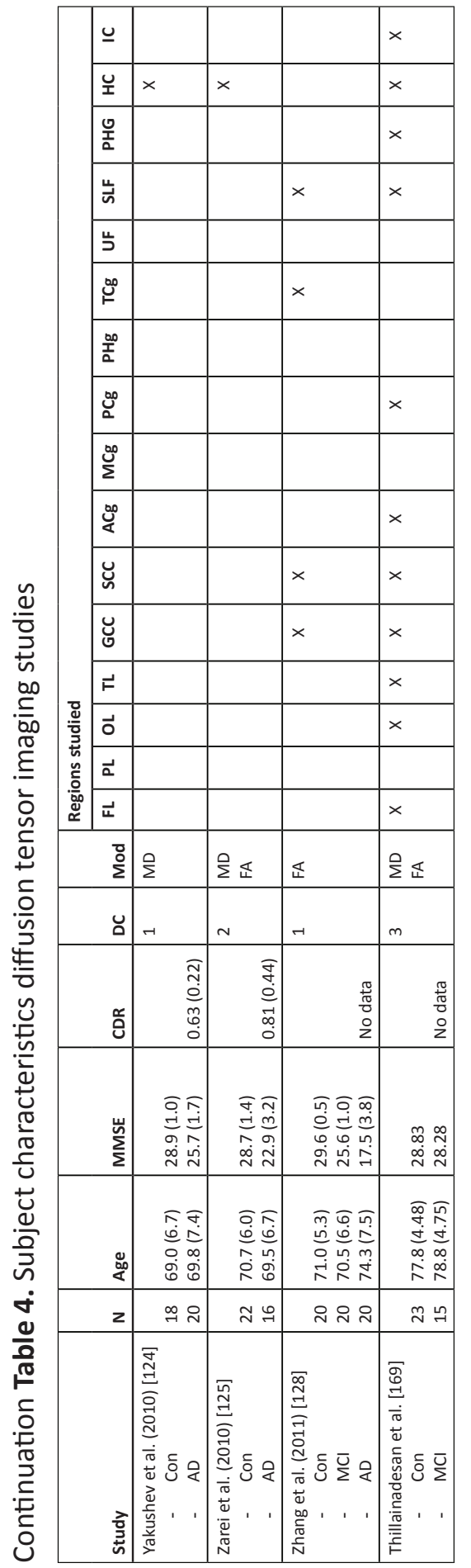

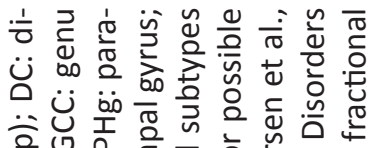

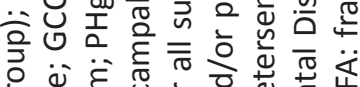

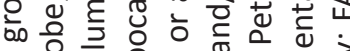

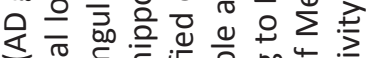

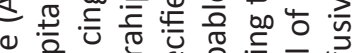

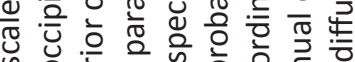
जั

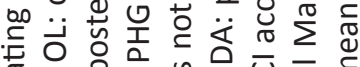

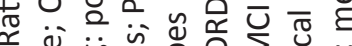

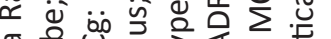

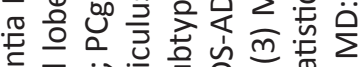

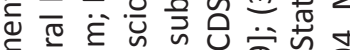

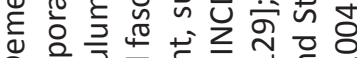

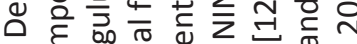

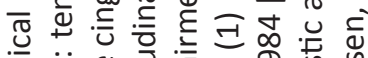

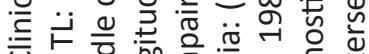
บ

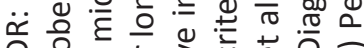
宅 으 헌

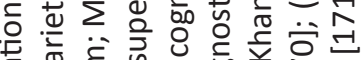
은 हैं⿰讠⿱士⿻尸丨 元

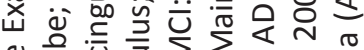
은 는 $\sum \sum \frac{0}{\overline{0}}$

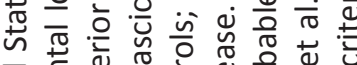
ত ธ

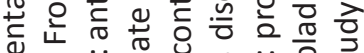

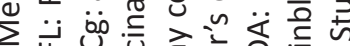

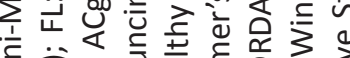

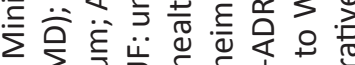

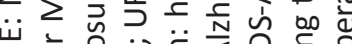

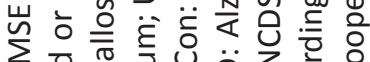

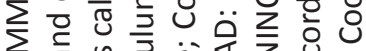

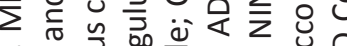

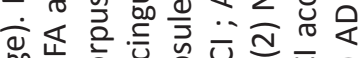

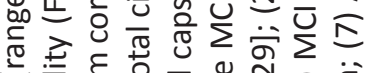

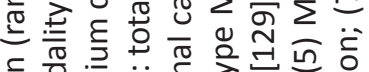
듀유ำ ๕ $\Sigma \frac{0}{2}$ थ

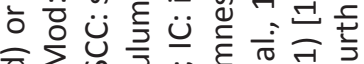
대 $\sum$ 잉 ᄃ

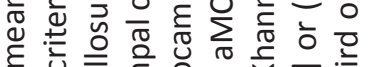

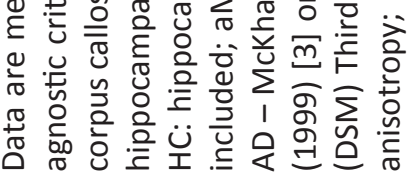


Table 5. Effect size DTI-fractional anisotropy studies

\begin{tabular}{|c|c|c|c|c|c|c|c|}
\hline & & Effect size & & Heterogeneity & & Public & ion Bias \\
\hline & & Hedges $\mathrm{g}(\mathrm{Cl})$ & $p$-value & Cochrane's Q & $p$-value & K-tau & $p$-value \\
\hline Controls vs $A D$ & $\begin{array}{l}\text { N studies } \\
\text { ( N Controls /N AD) }\end{array}$ & & & & & & \\
\hline Frontal Lobe & $15(309 / 265)$ & $0.70(0.35 ; 1.04)$ & 0.001 & 48.13 & $<0.001$ & 0.56 & 0.004 \\
\hline Parietal Lobe & $13(284 / 240)$ & $0.26(0.13 ; 0.66)$ & 0.19 & 51.04 & $<0.001$ & 0.38 & 0.08 \\
\hline Occipital Lobe & $14(298 / 244)$ & $0.11(-0.09 ; 0.32)$ & 0.29 & 10.11 & 0.52 & -0.39 & 0.09 \\
\hline Temporal Lobe & $18(357 / 314)$ & $0.57(0.29 ; 0.84)$ & $<0.001$ & 45.0 & $<0.001$ & 0.24 & 0.17 \\
\hline Hippocampus & $4(63 / 70)$ & $1.36(-0.16 ; 2.88)$ & 0.08 & 40.88 & $<0.001$ & 0.67 & 0.31 \\
\hline Total cingulum & $5(84 / 90)$ & $1.73(0.22 ; 3.23)$ & 0.02 & 63.33 & $<0.001$ & 0.80 & 0.09 \\
\hline $\mathrm{ACg}$ & $3(59 / 58)$ & $1.37(-0.30 ; 3.04)$ & 0.11 & 30.18 & $<0.001$ & 0.33 & 1.00 \\
\hline $\mathrm{MCg}$ & $3(43 / 44)$ & $0.42(-0.006 ; 0.85)$ & 0.05 & 0.12 & 0.94 & -0.33 & 1.00 \\
\hline PCg & $10(186 / 196)$ & $0.84(0.63 ; 1.05)$ & $<0.001$ & 7.11 & 0.63 & -0.16 & 0.60 \\
\hline PHg & $7(134 / 128)$ & $1.17(0.40 ; 1.95)$ & 0.003 & 22.88 & $<0.001$ & 0.80 & 0.09 \\
\hline Genu CC & $19(417 / 361)$ & $0.63(0.42 ; 0.83)$ & $<0.001$ & 31.29 & 0.03 & 0.39 & 0.02 \\
\hline Splenium CC & $22(467 / 417)$ & $1.10(0.65 ; 1.55)$ & $<0.001$ & 178.92 & $<0.001$ & 0.34 & 0.03 \\
\hline SLF & $7(125 / 136)$ & $0.77(0.15 ; 1.39)$ & 0.01 & 31.54 & $<0.001$ & 0.62 & 0.07 \\
\hline UF & $5(84 / 94)$ & $1.03(0.48 ; 1.58)$ & $<0.001$ & 11.68 & 0.02 & 1.00 & 0.03 \\
\hline Internal capsule & $9(136 / 139)$ & $0.09(-0.19 ; 0.37)$ & 0.54 & 1.81 & 0.94 & 0.14 & 0.76 \\
\hline Controls vs $\mathrm{MCl}$ & $\begin{array}{l}N \text { studies } \\
\text { ( } N \text { Controls /N MCl) }\end{array}$ & & & & & & \\
\hline Frontal Lobe & $10(444 / 345)$ & $0.26(-0.11 ; 0.62)$ & 0.17 & 37.26 & $<0.001$ & 0.02 & 1.00 \\
\hline Parietal Lobe & $8(196 / 177)$ & $0.02(-0.26 ; 0.29)$ & 0.90 & 10.31 & 0.17 & 0.50 & 0.11 \\
\hline Occipital Lobe & $9(434 / 335)$ & $0.18(-0.13 ; 0.49)$ & 0.25 & 5.47 & 0.37 & -0.47 & 0.26 \\
\hline Temporal Lobe & $14(517 / 412)$ & $0.38(0.11 ; 0.65)$ & 0.006 & 37.34 & $<0.001$ & 0.30 & 0.15 \\
\hline Hippocampus & $6(310 / 247)$ & $0.55(-0.16 ; 1.26)$ & 0.12 & 42.12 & $<0.001$ & 0.33 & 0.45 \\
\hline PHG & $3(270 / 189)$ & $0.06(-0.12 ; 0.25)$ & 0.52 & 0.39 & 0.84 & 1.00 & 0.30 \\
\hline $\mathrm{PCg}$ & $10(402 / 327)$ & $0.65(0.32 ; 0.98)$ & $<0.001$ & 29.47 & $<0.001$ & 0.38 & 0.15 \\
\hline PHg & $4(68 / 68)$ & $0.97(0.61 ; 1.32)$ & $<0.001$ & 0.09 & 0.99 & 0.00 & 1.00 \\
\hline Genu CC & $16(573 / 466)$ & $0.41(0.18 ; 0.64)$ & $<0.001$ & 37.74 & $<0.001$ & 0.27 & 0.16 \\
\hline Splenium CC & $17(602 / 504)$ & $0.44(0.25 ; 0.64)$ & $<0.001$ & 31.97 & 0.01 & 0.40 & 0.03 \\
\hline SLF & $4(285 / 214)$ & $0.66(-0.02 ; 1.34)$ & 0.06 & 17.45 & $<0.001$ & 1.00 & 0.09 \\
\hline UF & $3(47 / 51)$ & $0.26(-0.28 ; 0.81)$ & 0.35 & 3.71 & 0.16 & 1.00 & 0.30 \\
\hline$M C l$ vs $A D$ & $\begin{array}{l}\text { N studies } \\
\text { (N MCI/N AD) }\end{array}$ & & & & & & \\
\hline Frontal Lobe & $6(145 / 120)$ & $0.29(0.05 ; 0.54)$ & 0.02 & 2.67 & 0.75 & 0.07 & 1.00 \\
\hline Parietal Lobe & $6(167 / 146)$ & $-0.09(-0.32 ; 0.13)$ & 0.40 & 1.35 & 0.93 & 0.13 & 0.85 \\
\hline Occipital Lobe & $6(145 / 120)$ & $-0.07(-0.40 ; 0.26)$ & 0.69 & 1.65 & 0.80 & -0.1 & 1.00 \\
\hline Temporal Lobe & $10(212 / 195)$ & $0.27(0.03 ; 0.51)$ & 0.03 & 11.90 & 0.22 & -0.11 & 0.72 \\
\hline $\mathrm{PCg}$ & $6(120 / 123)$ & $0.37(0.12 ; 0.63)$ & 0.004 & 4.88 & 0.43 & 0.07 & 1.00 \\
\hline PHg & $4(68 / 68)$ & $0.46(-0.14 ; 1.06)$ & 0.13 & 8.44 & 0.04 & -0.67 & 0.31 \\
\hline Genu CC & $10(224 / 211)$ & $0.35(0.16 ; 0.54)$ & $<0.001$ & 4.69 & 0.86 & -0.2 & 1.00 \\
\hline Splenium CC & $12(274 / 256)$ & $0.51(0.11 ; 0.91)$ & 0.01 & 51.01 & $<0.001$ & 0.06 & 0.84 \\
\hline
\end{tabular}

$\mathrm{Cl}$ : Confidence interval; $\mathrm{MCl}$ : mild cognitive impairment; $\mathrm{AD}$ : Alzheimer's disease. Data shown in bold are statistically significant. ACg: anterior cingulum; $\mathrm{MCg}$ : middle cingulum; $\mathrm{PCg}$ : posterior cingulum; $\mathrm{PHg}$ : parahippocampal cingulum; UF: uncinate fasciculus; SLF: superior longitudinal fasciculus, PHG: parahippocampal gyrus. 
Table 6. Effect size DTI-mean diffusivity studies

\begin{tabular}{|c|c|c|c|c|c|c|c|}
\hline & & Effect size & & Heterogeneity & & Public & ion Bias \\
\hline & & Hedges $\mathrm{g}(\mathrm{Cl})$ & $p$-value & Cochrane's Q & $p$-value & K-tau & $p$-value \\
\hline Controls vs $A D$ & $\begin{array}{l}\text { N studies } \\
\text { (N Controls /N AD) }\end{array}$ & & & & & & \\
\hline Frontal Lobe & $10(165 / 176)$ & $-0.75(-1.24 ;-0.27)$ & 0.002 & 38 & $<0.001$ & -0.47 & 0.07 \\
\hline Parietal Lobe & $9(155 / 166)$ & $-1.03(-1.58 ;-0.49)$ & $<0.001$ & 38.73 & $<0.001$ & -0.61 & 0.03 \\
\hline Occipital Lobe & $8(123 / 139)$ & $-0.67(-1.00 ;-0.33)$ & $<0.001$ & 11.81 & 0.11 & 0.14 & 0.71 \\
\hline Temporal Lobe & $12(187 / 207)$ & $-0.89(-1.30 ;-0.48)$ & $<0.001$ & 37.76 & $<0.001$ & -0.27 & 0.24 \\
\hline Hippocampus & $7(125 / 131)$ & $-1.17(-1.81 ;-0.53)$ & $<0.001$ & 31.35 & $<0.001$ & -0.62 & 0.07 \\
\hline PCg & $9(156 / 164)$ & $-0.74(-0.97 ;-0.51)$ & $<0.001$ & 5.57 & 0.70 & -0.11 & 0.75 \\
\hline Genu CC & $12(226 / 216)$ & $-0.67(-0.98 ;-0.35)$ & $<0.001$ & 26.79 & 0.005 & -0.30 & 0.19 \\
\hline Splenium CC & $12(226 / 216)$ & $-0.94(-1.53 ;-0.35)$ & 0.002 & 81.10 & $<0.001$ & -0.36 & 0.12 \\
\hline UF & $4(65 / 76)$ & $-0.72(-1.27 ;-0.17)$ & 0.01 & 7.32 & 0.06 & 0.00 & 1.00 \\
\hline Internal capsule & $7(118 / 118)$ & $-0.66(-1.19 ;-0.13)$ & 0.01 & 21.66 & $<0.001$ & -0.43 & 0.23 \\
\hline Controls vs $\mathrm{MCl}$ & $\begin{array}{l}\text { studies } \\
\text { (N Controls /N MCI) }\end{array}$ & & & & & & \\
\hline Frontal Lobe & $7(325 / 249)$ & $-0.32(-0.62 ; 0.001)$ & 0.05 & 10.88 & 0.09 & -0.24 & 0.55 \\
\hline Parietal Lobe & $5(77 / 786)$ & $-0.69(-1.09 ;-0.28)$ & 0.01 & 6.12 & 0.19 & 0.00 & 1.00 \\
\hline Occipital Lobe & $6(315 / 244)$ & $-0.19(-0.55 ;-0.17)$ & 0.30 & 11.49 & 0.04 & 0.20 & 0.71 \\
\hline Temporal Lobe & $10(379 / 289)$ & $-0.40(-0.56 ;-0.24)$ & $<0.001$ & 7.41 & 0.60 & -0.24 & 0.37 \\
\hline Hippocampus & $7(344 / 276)$ & $-1.00(-1.62 ;-0.40)$ & 0.001 & 46.74 & $<0.001$ & -0.81 & 0.02 \\
\hline PHG & $3(270 / 189)$ & $-2.52(-6.62 ; 1.58)$ & 0.23 & 246.13 & $<0.001$ & 0.33 & 1.00 \\
\hline PCg & $7(342 / 257)$ & $-0.26(-0.43 ;-0.10)$ & 0.002 & 5.16 & 0.52 & -0.62 & 0.07 \\
\hline Genu CC & $10(379 / 289)$ & $-0.43(-0.69 ;-0.17)$ & 0.001 & 14.96 & 0.09 & -0.02 & 1.00 \\
\hline Splenium CC & $10(379 / 289)$ & $-0.46(-0.76 ;-0.15)$ & 0.004 & 20.79 & 0.01 & -0.21 & 0.53 \\
\hline$M C l$ vs $A D$ & $\begin{array}{l}N \text { studies } \\
\text { (N MCI/N AD) }\end{array}$ & & & & & & \\
\hline Temporal Lobe & $6(89 / 113)$ & $-0.17(-0.45 ; 0.12)$ & 0.25 & 3.74 & 0.59 & 0.13 & 0.85 \\
\hline Hippocampus & $3(68 / 70)$ & $-0.33(-0.77 ;-0.11)$ & 0.14 & 3.39 & 0.18 & 1.00 & 0.30 \\
\hline $\mathrm{PCg}$ & $5(88 / 99)$ & $-0.40(-0.70 ;-0.11)$ & 0.007 & 2.12 & 0.70 & 0.00 & 1.00 \\
\hline Genu CC & $6(89 / 113)$ & $-0.32(-0.61 ;-0.03)$ & 0.03 & 2.61 & 0.76 & 0.07 & 1.00 \\
\hline Splenium CC & $6(89 / 113)$ & $-0.04(-0.07 ; 0.003)$ & 0.03 & 8.54 & 0.13 & 0.00 & 1.00 \\
\hline
\end{tabular}

$\mathrm{Cl}$ : Confidence interval; $\mathrm{MCl}$ : mild cognitive impairment; $\mathrm{AD}$ : Alzheimer's disease. Data shown in bold are statistically significant. PCg: posterior cingulum, UF: uncinate fasciculus; PHG: parahippocampal gyrus. 

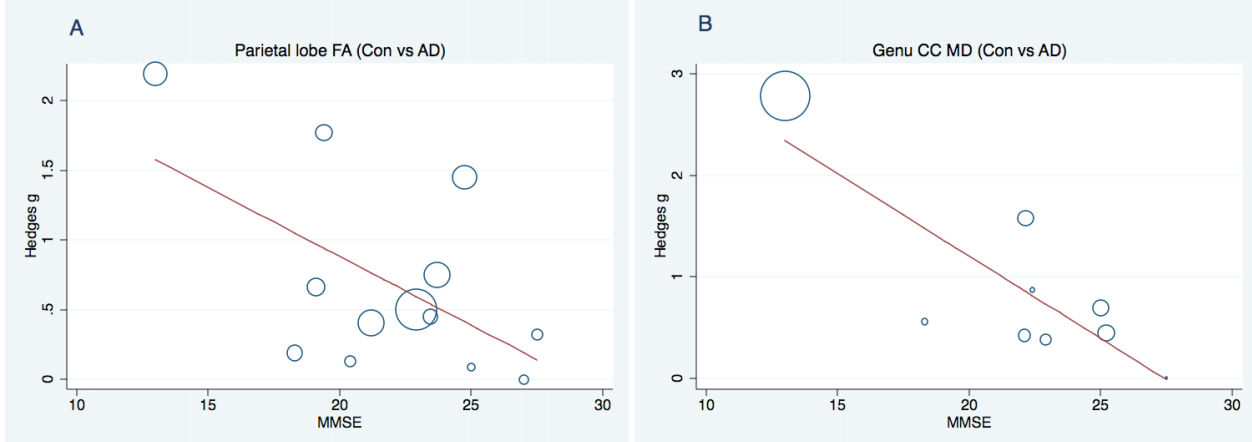

Figure 1. Meta-regression with MMSE as predictor and absolute effect size as outcome measure. (A) FA values of the parietal lobe between controls and subjects with Alzheimer's disease $(A D) ; B=-0.1 \pm 0.04, p=0.04$. (B) MD of the genu corpus callosum $(C C)$ between controls and subjects with $A D ; B=-0.2 \pm 0.05, p=0.01$. 

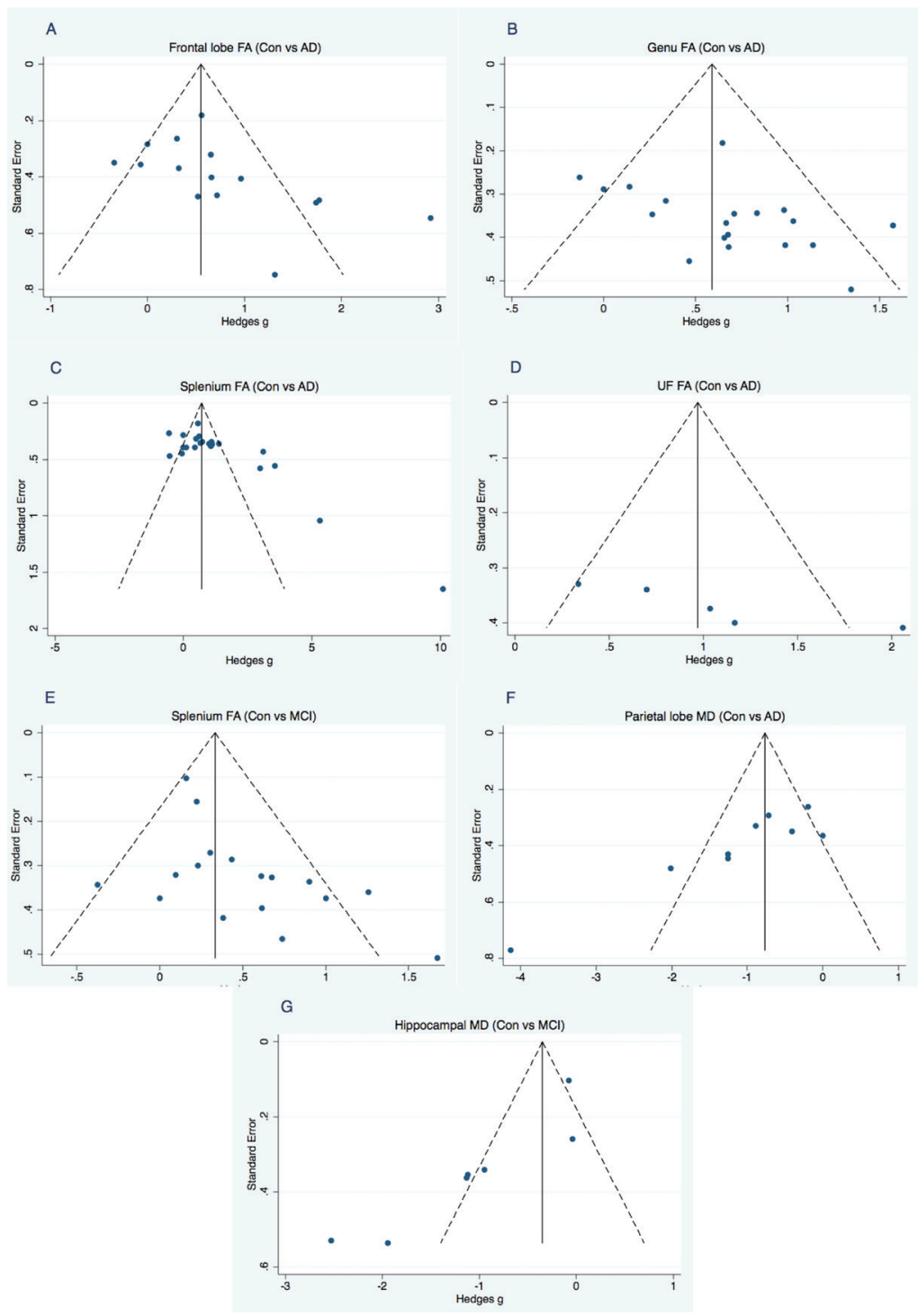

Figure 2. Funnel plots of standard errors plotted against effect sizes in order to identify publication bias. (A) Comparison between controls and subjects with Alzheimer's disease (AD) for frontal lobe fractional anisotropy (FA). Begg and Mazumdar tau $=0.56, p=0.004$. (B) Comparison between controls and AD for FA of the genu corpus callosum (CC). Begg and Mazumdar tau $=0.39, p=0.02$. (C) Comparison between controls and AD for FA of the splenium CC. Begg and Mazumdar tau $=0.34, p=0.03$. (D) Comparison between controls and AD for FA of the uncinate fasciculus. Begg and Mazumdar tau $=1.00, p=0.03$. (E) Comparison between controls and subjects with $\mathrm{MCl}$ for FA of the splenium CC. Begg and Mazumdar tau $=0.40, p=0.03$. (F) Comparison between controls and AD for mean diffusivity (MD) of parietal WM. Begg and Mazumdar tau $=-0.61, p=0.03$. (G) Comparison between controls and subjects with $\mathrm{MCl}$ for MD of the hippocampus. Begg and Mazumdar tau $=-0.81, p=0.02$. 


\section{SUPPLEMENTAL MATERIAL}

\section{Supplementary table 1. Technical specifications medial temporal lobe studies. Technical details of MTL studies included in the meta-analysis.}

\begin{tabular}{|c|c|c|c|c|}
\hline Study & $\begin{array}{c}\text { Total } \\
\mathbf{N}\end{array}$ & $\begin{array}{c}\text { Field } \\
\text { strength } \\
\text { (Tesla) }\end{array}$ & $\begin{array}{l}\text { Slice thick- } \\
\text { ness (in } \mathrm{mm} \text { ) }\end{array}$ & $\begin{array}{c}\text { Volumetric measurement of MTL } \\
\text { structures }\end{array}$ \\
\hline Pitkänen et al. (1996) [1] & 131 & 1.5 & 1.5 & Manual tracing \\
\hline Convit et al. (1997) [2] & 76 & 1.5 & 4 & Manual tracing \\
\hline Kaye et al. (1997) [3] & 30 & 1.5 & 2.5 & Manual tracing \\
\hline Mori et al. (1997) [4] & 58 & 1.5 & 1.5 & Semi-automated tracing \\
\hline Jack et al. (1998) [5] & 48 & 1.5 & 1.6 & Manual tracing \\
\hline Juottonen et al. (1998) [6] & 62 & 1.5 & 1.75 & Manual tracing \\
\hline Krasuski et al. (1998) [7] & 34 & 1.5 & 0.5 & Manual tracing \\
\hline Laakso et al. (1998) [8] & 139 & 1.5 & 1.5 & Manual tracing \\
\hline Visser et al. (1999) [9] & 25 & 0.6 & 5 & Manual tracing \\
\hline Barber et al. (2000) [10] & 48 & 1 & 1 & Manual tracing \\
\hline de Toledo-Morrell (2000) [11] & 48 & 1.5 & 5 & Manual tracing \\
\hline Laakso et al. (2000) [12] & 60 & 1.5 & 2 & Manual tracing \\
\hline Xu et al. (2000) [13] & 90 & 1.5 & 1.6 & Manual tracing \\
\hline Du et al. (2001) [14] & 105 & 1.5 & 1.4 & Manual tracing \\
\hline De Santi et al. (2001) [15] & 38 & 1.5 & 1.3 & Manual tracing \\
\hline Goncharova et al. (2001) [16] & 50 & 1.4 & 5 & Manual tracing \\
\hline Wolf et al. (2001) [17] & 39 & 1.5 & 1.5 & Manual tracing \\
\hline Bottino et al. (2002) [18] & 59 & 1.5 & 0.6 & Manual tracing \\
\hline Hsu et al. (2002) [19] & 60 & 1.5 & 1 & Manual tracing \\
\hline Killiany et al. (2002) [20] & 138 & 1.5 & 1.5 & Manual tracing \\
\hline Mega et al. (2002) [21] & 20 & 1.5 & 1.5 & Manual tracing \\
\hline Pantel et al. (2002) [22] & 55 & 1.5 & - & Manual tracing \\
\hline Du et al. (2003) [23] & 44 & 1.5 & 1.4 & Manual tracing \\
\hline Du et al. (2004) [24] & 45 & 1.5 & 1.4 & Manual tracing \\
\hline Pennanen et al. (2004) [25] & 172 & 1.5 & 2 & Manual tracing \\
\hline Testa et al. (2004) [26] & 52 & 1.5 & 1.3 & VBM + manual tracing \\
\hline Müller et al. (2005) [27] & 36 & 1.5 & 1 & Manual tracing \\
\hline Bastos-Leite et al. (2006) [28] & 36 & 1.5 & 1.5 & N.A. \\
\hline Kalus et al. (2006) [29] & 30 & 1.5 & 1 & Manual tracing \\
\hline Teipel et al. (2006) [30] & 56 & 1.5 & 1.2 & Manual tracing \\
\hline
\end{tabular}




\begin{tabular}{|c|c|c|c|c|}
\hline Study & $\begin{array}{l}\text { Total } \\
\mathbf{N}\end{array}$ & $\begin{array}{c}\text { Field } \\
\text { strength } \\
\text { (Tesla) }\end{array}$ & $\begin{array}{l}\text { Slice thick- } \\
\text { ness (in mm) }\end{array}$ & $\begin{array}{l}\text { Volumetric measurement of MTL } \\
\text { structures }\end{array}$ \\
\hline Uotani et al. (2006) [31] & 60 & - & 5 & Manual tracing \\
\hline van de Pol et al. (2006) [32] & 176 & 1.5 & 1.5 & Manual tracing \\
\hline Barnes et al. (2007) [33] & 30 & 1.5 & 1.5 & Manual tracing \\
\hline Meyer et al. (2007) [34] & 101 & 1.5 & - & Manual tracing \\
\hline Ridha et al. (2007) [35] & 73 & 1.5 & 1.5 & Manual tracing \\
\hline Slavin et al. (2007) [36] & 35 & 4 & 1.5 & Manual tracing \\
\hline Colliot et al. (2008) [37] & 74 & 1.5 & 1.5 & Automated segmentation \\
\hline Delano-Wood (2008) [38] & 40 & 1.5 & 3.8 & Manual tracing \\
\hline Duara et al. (2008) [39] & 261 & 1.5 & 1.5 & N.A. \\
\hline Kenny et al. (2008) [40] & 63 & 1.5 & 1.6 & Manual tracing \\
\hline Appel et al. (2009) [41] & 192 & 1.5 & 1.5 & N.A. \\
\hline Bai et al. (2009) [42] & 62 & 1.5 & 2 & VBM + semi-automated tracing \\
\hline Cho et al. (2009) [43] & 60 & 1.5 & - & N.A. \\
\hline Feczko et al. (2009) [44] & 76 & 1.5 & 1.25 & Automated segmentation \\
\hline Ferrarini et al. (2009) [45] & 130 & 1 & 1.3 & Manual tracing \\
\hline Henneman et al. (2009) [46] & 75 & 1.5 & 1.5 & N.A. \\
\hline Henneman et al. (2009) [47] & 142 & 1.5 & 1.5 & Automated segmentation \\
\hline Hyun et al. (2009) [48] & 60 & 1.5 & - & N.A. \\
\hline Jauhiainen et al. (2009) [49] & 50 & 1.5 & 1 & Manual tracing \\
\hline Lee et al. (2009) [50] & 215 & 1.5 & - & Semi-automated tracing \\
\hline Loewenstein et al. (2009) [51] & 234 & 1.5 & 1.5 & N.A. \\
\hline Morra et al. (2009) [52] & 400 & 1.5 & 1.2 & Automated segmentation \\
\hline Rogalski et al. (2009) [53] & 28 & 1.5 & 1.6 & Manual tracing \\
\hline Bird et al. (2010) [54] & 38 & 1.5 & 1.5 & N.A. \\
\hline Bouwman et al. (2010) [55] & 348 & 1 & 1.5 & N.A. \\
\hline Cherubini et al. (2010) [56] & 90 & 3 & 1 & Automated segmentation \\
\hline Choo et al. (2010) [57] & 56 & 3 & 1.4 & Manual tracing \\
\hline Desikan et al. (2010) [58] & 724 & 1.5 & - & Automated segmentation \\
\hline Jhoo et al. (2010) [59] & 51 & 3 & 1.4 & Manual tracing \\
\hline Johnson et al. (2010) [60] & 59 & 3 & 1 & Automated segmentation \\
\hline Lehmann et al. (2010) [61] & 20 & 1.5 & 1.5 & Manual tracing \\
\hline Liu et al. (2010) [62] & 291 & 1.5 & 1.2 & Automated segmentation \\
\hline Luckhaus et al. (2010) [63] & 57 & 1.5 & 1.8 & Manual tracing \\
\hline Mueller et al. (2010) [63] & 91 & 4 & 2 & Manual tracing \\
\hline
\end{tabular}




\begin{tabular}{lcccc}
\hline Study & $\begin{array}{c}\text { Total } \\
\text { N }\end{array}$ & $\begin{array}{c}\text { Field } \\
\text { strength } \\
\text { (Tesla) }\end{array}$ & $\begin{array}{c}\text { Slice thick- } \\
\text { ness (in mm) }\end{array}$ & $\begin{array}{c}\text { Volumetric measurement of MTL } \\
\text { structures }\end{array}$ \\
\hline Pengas et al. (2010) [64] & 52 & 1.5 & 1.8 & Manual tracing \\
Ryu et al. (2010) [65] & 37 & 1.5 & 1 & Automated segmentation \\
Sanchez-Benavides et al. (2010) & 78 & 1.5 & 1.5 & Semi-automated tracing \\
[66] & 677 & 1.5 & - & Semi-automated tracing \\
Schott et al. (2010) [67] & 300 & 1.5 & 1.2 & Manual tracing \\
Westman et al. (2010) [68] & 38 & 1.5 & 1 & Manual tracing \\
Yakushev et al. (2010) [69] & 38 & 1.5 & 1 & Automated segmentation \\
Zarei et al. (2010) [70] & 54 & 3 & 1 & Manual tracing \\
Echavarri et al. (2011) [71] & 74 & 1.5 & 1.5 & Semi-automated tracing \\
Jacobs et al. (2011) [72] & 39 & 1 & 1.3 & Automated segmentation \\
Prestia et al. (2011) [73] & 389 & 3 & 1 & Manual tracing \\
Zhang et al. (2011) [74] & & &
\end{tabular}

MTL: Medial Temporal Lobe; VBM: Voxel Based Morphometry. N.A.: not applicable (only qualitative MTA-rating was provided in the study). 


\section{Supplementary table 2. Technical specifications diffusion tensor imaging studies}

Technical details of DTI studies included in the meta-analysis.

\begin{tabular}{|c|c|c|c|c|c|c|c|}
\hline Study & $\begin{array}{c}\text { Total } \\
\mathbf{N}\end{array}$ & $\begin{array}{c}\text { Field } \\
\text { strength } \\
\text { (Tesla) }\end{array}$ & $\begin{array}{l}\text { Number of } \\
\text { directions }\end{array}$ & $\begin{array}{l}\text { Voxel size } \\
\text { (mm) }\end{array}$ & $\begin{array}{l}\text { b-value } \\
\left(\mathrm{s} / \mathrm{mm}^{2}\right)\end{array}$ & NEX & DTI modality \\
\hline Rose et al. (2000) [75] & 20 & 1.5 & 6 & $1.2 \times 2.3 \times 6.0$ & 875 & 1 & $\begin{array}{l}\text { Manual placement of standard } \\
\text { ROI's }\end{array}$ \\
\hline Bozzali et al. (2002) [76] & 26 & 1.5 & 8 & $2.0 \times 2.0 \times 5.0$ & 1044 & 1 & Automated segmentation \\
\hline Stahl et al. (2003) [77] & 19 & 1.5 & 6 & $1.8 \times 1.8 \times 3.6$ & 1000 & 10 & VBM \\
\hline Fellgiebel et al. (2004) [78] & 43 & 1.5 & 6 & Slice thickness 5.0 & 900 & 6 & $\begin{array}{l}\text { Manual placement of standard } \\
\text { ROI's }\end{array}$ \\
\hline Head et al. (2004) [79] & 50 & 1.5 & 7 & $2.5 \times 2.5 \times 4.0$ & 1005 & 4 & Manual tracing \\
\hline Sugihara et al. (2004) [80] & 30 & 1.5 & 6 & Slice thickness 5.0 & 1000 & 1 & $\begin{array}{l}\text { Manual placement of standard } \\
\text { ROI's }\end{array}$ \\
\hline Choi et al. (2005) [81] & 20 & 1.5 & 6 & $1.9 \times 1.9 \times 5.0$ & 1000 & 4 & $\begin{array}{l}\text { Manual placement of standard } \\
\text { ROI's }\end{array}$ \\
\hline Fellgiebel et al. (2005) [82] & 63 & 1.5 & 6 & $1.8 \times 1.8 \times 5.0$ & 900 & 4 & Tractography \\
\hline Duan et al. (2006) [83] & 28 & 1.5 & 25 & $1.9 \times 1.9 \times 5.0$ & 1000 & 1 & $\begin{array}{l}\text { Manual placement of standard } \\
\text { ROI's }\end{array}$ \\
\hline Naggara et al. (2006) [84] & 24 & 1.5 & 25 & $1.8 \times 1.8 \times 3.0$ & 1000 & 2 & $\begin{array}{l}\text { Manual placement of standard } \\
\text { ROI's }\end{array}$ \\
\hline Rose et al. (2006) [85] & 34 & 1.5 & 6 & Slice thickness 2.5 & 1100 & 1 & VBM \\
\hline Taoka et al. (2006) [86] & 30 & 1.5 & 6 & $1.8 \times 1.8 \times 6.3$ & 1000 & 6 & Tractography \\
\hline Chen et al. (2007) [87] & 20 & 1.5 & 25 & $1.9 \times 1.9 \times 3.0$ & 1000 & 1 & Automated segmentation \\
\hline Firbank et al. (2007) [88] & 30 & 1.5 & 6 & $2.6 \times 1.9 \times 6.0$ & 4000 & 1 & $\begin{array}{l}\text { Manual placement of standard } \\
\text { ROI's }\end{array}$ \\
\hline Huang and Auchus (2007) [89] & 18 & 1.5 & 12 & $1.9 \times 1.9 \times 3.0$ & 1000 & 1 & $\begin{array}{l}\text { Manual placement of standard } \\
\text { ROI's }\end{array}$ \\
\hline Müller et al. (2007) [90] & 36 & 1.5 & 6 & $1.8 \times 1.8 \times 5.0$ & 900 & 1 & $\begin{array}{l}\text { Manual placement of standard } \\
\text { ROI's }\end{array}$ \\
\hline Stahl et al. (2007) [91] & 50 & 1.5 & 6 & $1.8 \times 1.8 \times 3.6$ & 1000 & 10 & $\begin{array}{l}\text { Manual placement of standard } \\
\text { ROI's }\end{array}$ \\
\hline Zhang et al. (2007) [92] & 52 & 1.5 & 6 & $2.3 \times 2.3 \times 5.0$ & 1000 & 1 & $\begin{array}{l}\text { Manual placement of standard } \\
\text { ROI's }\end{array}$ \\
\hline Cho et al. (2008) [93] & 22 & 1.5 & 25 & $2.0 \times 2.0 \times 4.0$ & 1000 & 1 & $\begin{array}{l}\text { Manual placement of standard } \\
\text { ROI's }\end{array}$ \\
\hline Delano-Wood (2008) [38] & 40 & 1.5 & 42 & $3.8 \times 3.8 \times 2.8$ & 1990 & 7 & $\begin{array}{c}\text { Tractography + manual place- } \\
\text { ment of ROI's }\end{array}$ \\
\hline Ding et al. (2008) [94] & 37 & 1.5 & 25 & $1.9 \times 1.9 \times 5.0$ & 1000 & 1 & $\begin{array}{l}\text { Manual placement of standard } \\
\text { ROI's }\end{array}$ \\
\hline Fujie et al. (2008) [95] & 32 & 3 & 12 & $1.8 \times 1.8 \times 3.0$ & 700 & 1 & Tractography \\
\hline
\end{tabular}




\begin{tabular}{|c|c|c|c|c|c|c|c|}
\hline Study & $\begin{array}{c}\text { Total } \\
\mathbf{N}\end{array}$ & $\begin{array}{c}\text { Field } \\
\text { strength } \\
\text { (Tesla) }\end{array}$ & $\begin{array}{l}\text { Number of } \\
\text { directions }\end{array}$ & $\begin{array}{c}\text { Voxel size } \\
\text { (mm) }\end{array}$ & $\begin{array}{l}\text { b-value } \\
\left(\mathrm{s} / \mathrm{mm}^{2}\right)\end{array}$ & NEX & DTI modality \\
\hline Parente et al. (2008) [96] & 61 & 1.5 & 6 & $1.8 \times 1.8 \times 6.5$ & 1000 & 3 & $\begin{array}{l}\text { Manual placement of standard } \\
\text { ROI's }\end{array}$ \\
\hline Shim et al. (2008) [97] & 38 & 1.5 & 25 & $2.0 \times 2.0 \times 5.0$ & 1000 & 1 & $\begin{array}{l}\text { Manual placement of standard } \\
\text { ROI's }\end{array}$ \\
\hline Ukmar et al. (2008) [98] & 47 & 1.5 & 6 & $2.5 \times 1.9 \times 6.0$ & 1000 & 1 & $\begin{array}{l}\text { Manual placement of standard } \\
\text { ROI's }\end{array}$ \\
\hline Yasmin et al. (2008) [99] & 38 & 1 & 12 & $1.8 \times 1.8 \times 3.0$ & 700 & 6 & Tractography \\
\hline Bai et al. (2009) [100] & 44 & 1.5 & 25 & $1.9 \times 1.9 \times 4.0$ & 1000 & 2 & $\begin{array}{l}\text { Manual placement of standard } \\
\text { ROI's }\end{array}$ \\
\hline Chen et al. (2009) [101] & 40 & 1.5 & 25 & $1.9 \times 1.9 \times 5.0$ & 1000 & 2 & $\begin{array}{l}\text { Manual placement of standard } \\
\text { ROI's }\end{array}$ \\
\hline Chen et al. (2009) [102] & 60 & 1.5 & 25 & $1.9 \times 1.9 \times 5.0$ & 1000 & 2 & $\begin{array}{c}\text { Manual placement of standard } \\
\text { ROI's }\end{array}$ \\
\hline Kiuchi et al. (2009) [103] & 49 & 1.5 & 6 & $1.8 \times 1.8 \times 3.0$ & 1000 & 6 & Tractography \\
\hline Liu et al. (2009) [104] & 63 & 1.5 & 30 & $1.7 \times 1.7 \times 5.0$ & 1000 & 1 & $\begin{array}{c}\text { TBSS + manual placement of } \\
\text { ROI's }\end{array}$ \\
\hline Mielke et al. (2009) [105] & 75 & 3 & 32 & $2.2 \times 2.2 \times 2.2$ & 700 & 2 & Tractography + manual tracing \\
\hline Nakata et al. (2009) [106] & 41 & 1 & 12 & $1.8 \times 1.8 \times 3.0$ & 700 & 6 & Tractography \\
\hline Rogalski (2009) [53] & 28 & 1.5 & 24 & $2.0 \times 2.0 \times 3.0$ & 800 & 6 & $\begin{array}{l}\text { Manual placement of standard } \\
\text { ROI's }\end{array}$ \\
\hline Stricker et al. (2009) [107] & 30 & 3 & 15 & $1.9 \times 1.9 \times 3.0$ & 1500 & 4 & $\begin{array}{c}\text { TBSS + manual placement of } \\
\text { ROI's }\end{array}$ \\
\hline Wang et al. (2009) [108] & 20 & 3 & 16 & $1.9 \times 1.9 \times 2.0$ & 1000 & 1 & $\begin{array}{l}\text { Manual placement of standard } \\
\text { ROI's }\end{array}$ \\
\hline Zarei et al. (2009) [109] & 38 & 1.5 & 60 & $2.0 \times 2.0 \times 2.0$ & 700 & 1 & TBSS, tractography \\
\hline Zhang et al. (2009) [110] & 37 & 4 & 6 & $2.0 \times 2.0 \times 3.0$ & 800 & 4 & Tractography \\
\hline Catheline et al. (2010) [111] & 30 & 1.5 & 6 & $2.4 \times 2.4 \times 2.5$ & 800 & 1 & Tractography \\
\hline Cherubini et al. (2010) [56] & 90 & 3 & 12 & $1.5 \times 1.5 \times 2.0$ & 1000 & 1 & Automated segmentation \\
\hline Choo et al. (2010) [57] & 56 & 3 & 25 & $0.94 \times 0.94 \times 0.94$ & 1000 & 1 & $\begin{array}{l}\text { Tractography, manual placement } \\
\text { of VOI's }\end{array}$ \\
\hline Hong et al. (2010) [112] & 30 & 1.5 & 25 & Slice thickness 4.0 & 1000 & 1 & $\begin{array}{c}\text { Manual placement of standard } \\
\text { ROI's }\end{array}$ \\
\hline Jhoo et al. (2010) [59] & 51 & 3 & 25 & $0.94 \times 0.94 \times 0.94$ & 1000 & 1 & $\begin{array}{c}\text { Tractography, manual placement } \\
\text { of VOI's }\end{array}$ \\
\hline
\end{tabular}

NEX: Number of excitations; ROI: Region of interest; VOI: Volume of Interest; TBSS: Tract-based Spatial Statistics. 


\section{Supplementary table 3. Significant univariate regressions for DTI effect sizes and technical parameters}

Meta-regression indicated that a number of technical parameters were associated with effect size (ES). An increasing number of directions was associated with a higher ES. A possible explanation for this finding is that with an increasing number of directions contrast of FA/MD maps improves. The contrast-to-signal variance ratio between the main WM and the surrounding regions significantly increases as number of directions increases allowing a better delineation of the gray-white matter junction and thus a more precise FA determination $[120,121]$. The ES of splenium FA seemed to increase with decreasing $b$-value and increasing field strength ( $M C l$ vs $A D)$. This could be explained by the fact that a higher field strength provides better spatial and contrast resolution compared to a lower field strength. In one study FA values were significantly higher at 3.0 Tesla compared with 1.5 Tesla [122]. In general, a higher b-value leads to lower signal-to-noise levels and higher FA values $[89,124]$. Our finding is therefore somewhat counterintuitive. However, there seems to be a lot of variability between studies regarding the effect of b-value on FA, since other studies found that FA values were not dependent on changes in b-value [123, 124]. The ES of parietal MD (controls vs $\mathrm{MCl}$ ) and genu CC MD (MCI vs $A D$ ) seems to increase with increasing voxel size. The ES of hippocampal MD on the contrary, seems to increase with a decrease in voxel size. A possible explanation is that in broader structures (e.g. corpus callosum) a higher voxel size can clearly reveal possible differences whereas in smaller structures (e.g. hippocampus) a smaller voxel size is needed for better resolution. Current evidence indicates that in general, mean diffusivity values are not affected by voxel size [125]. 


\section{Supplementary table 3}

\begin{tabular}{|c|c|c|c|c|c|}
\hline & & \multirow{2}{*}{$\begin{array}{l}\text { Effect size } \\
\text { Hedges g (Cl) }\end{array}$} & \multirow[b]{2}{*}{$p$-value } & \multicolumn{2}{|c|}{ Significant univariate regressions } \\
\hline & & & & DTI parameter & $p$-value \\
\hline \multicolumn{6}{|l|}{ FA } \\
\hline Controls vs $A D$ & $N$ studies ( $N$ Controls /N AD) & & & & \\
\hline Occipital Lobe & $14(298 / 244)$ & $0.11(-0.09 ; 0.32)$ & 0.29 & Directions & 0.05 \\
\hline Total cingulum & $5(84 / 90)$ & $1.73(0.22 ; 3.23)$ & 0.02 & Field srength & 0.008 \\
\hline ACC & $3(59 / 58)$ & $1.37(-0.30 ; 3.04)$ & 0.11 & $\begin{array}{l}\text { Directions } \\
\text { B-value (-) }\end{array}$ & $\begin{array}{l}0.04 \\
0.04\end{array}$ \\
\hline Splenium CC & $22(467 / 417)$ & $1.10(0.65 ; 1.55)$ & $<0.001$ & Directions & 0.008 \\
\hline Controls vs $\mathrm{MCl}$ & N studies (N Controls /N MCI) & & & & \\
\hline PCC & $10(402 / 327)$ & $0.65(0.32 ; 0.98)$ & $<0.001$ & Directions & 0.006 \\
\hline$M C l$ vs $A D$ & $N$ studies (N MCI/N AD) & & & & \\
\hline Splenium CC & $12(274 / 256)$ & $0.51(0.11 ; 0.91)$ & 0.01 & $\begin{array}{l}\text { Field srength } \\
\text { B-value (-) }\end{array}$ & $\begin{array}{l}0.03 \\
0.01\end{array}$ \\
\hline \multicolumn{6}{|l|}{ MD } \\
\hline Controls vs $A D$ & $N$ studies ( $N$ Controls /N AD) & & & & \\
\hline Frontal Lobe & $10(165 / 176)$ & $-0.75(-1.24 ;-0.27)$ & 0.002 & Directions & 0.053 \\
\hline Parietal Lobe & $9(155 / 166)$ & $-1.03(-1.58 ;-0.49)$ & $<0.001$ & Directions & 0.009 \\
\hline Splenium CC & $12(226 / 216)$ & $-0.94(-1.53 ;-0.35)$ & 0.002 & Directions & 0.04 \\
\hline Internal capsule & $7(118 / 118)$ & $-0.66(-1.19 ;-0.13)$ & 0.01 & Directions & 0.02 \\
\hline Controls vs $\mathrm{MCl}$ & N studies (N Controls /N MCI) & & & & \\
\hline Parietal Lobe & $5(77 / 786)$ & $-0.69(-1.09 ;-0.28)$ & 0.01 & Voxel size (+) & 0.04 \\
\hline$M C l$ vs $A D$ & $N$ studies (N MCI/N AD) & & & & \\
\hline Hippocampus & $3(68 / 70)$ & $-0.33(-0.77 ;-0.11)$ & 0.14 & $\begin{array}{l}\text { Field strength } \\
\text { Voxel size (-) }\end{array}$ & $\begin{array}{l}0.06 \\
0.06\end{array}$ \\
\hline Genu CC & $6(89 / 113)$ & $-0.32(-0.61 ;-0.03)$ & 0.03 & Voxel size (+) & 0.01 \\
\hline
\end{tabular}

$\mathrm{Cl}$ : Confidence interval; $\mathrm{MCl}$ : mild cognitive impairment; AD: Alzheimer's disease. Data shown in bold are statistically significant. PCC: posterior cingulum; PHC: parahippocampal cingulum; UF: uncinate fasciculus; SLF: superior longitudinal fasciculus.

Regression: + or $-=$ polarity of regression coefficient/slope. 



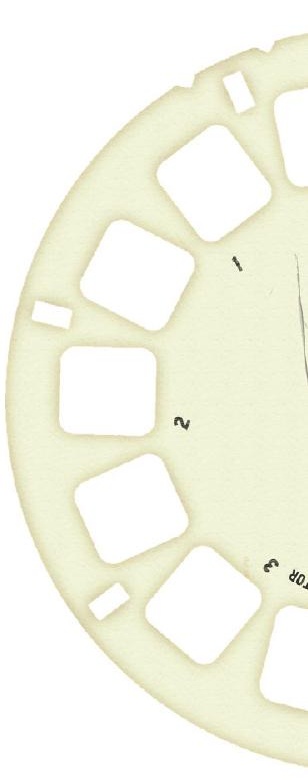




\section{Chapter 6}

Reduced callosal white matter integrity surpasses cerebrospinal fluid and atrophy markers as predictor of decline in subjects with $\mathrm{MCl}$ : a combined volumetry and DTI study

$\uparrow^{\prime}$

,

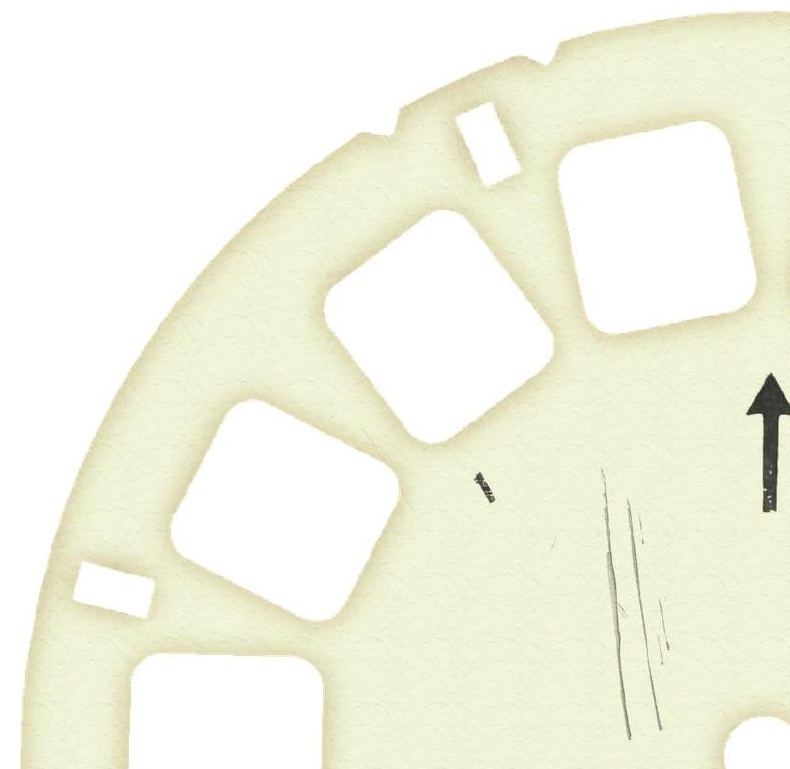

N

Lies Clerx, Heidi I.L. Jacobs, Ed H.B.M. Gronenschild, Walter H. Backes, Marcel G.M. Olde Rikkert, Jeroen A.H.R. Claassen, Frederik Barkhof, Maja Binnewijzend, Mark A. van Buchem, Jeroen van der Grond, Pieter-Jelle Visser, Frans R.J. Verhey and Pauline Aalten 


\section{ABSTRACT}

Background: Microstructural damage of white matter (WM) tracts is a prominent feature of Alzheimer's disease (AD). To date, it is still unknown whether subtle abnormalities in WM tissue microstructure precede volumetric alterations in gray matter (GM) and how alterations in both tissue-types are related to each other. Methods: In 3 memory clinics, we recruited 57 patients with mild cognitive impairment $(\mathrm{MCl})$ and 35 patients with mild $A D$, and combined these with data of 18 healthy controls (Con). The discriminative ability of atrophy and diffusion tensor imaging (DTI) was explored in 6 regions-of-interest: hippocampus, parahippocampal gyrus, posterior cingulum, isthmus of the cingulum and genu and splenium of the corpus callosum. Effect sizes (ES) were calculated in order to detect the measurement with the highest diagnostic potential. The value of both DTI and volumetry for predicting decline in $\mathrm{MCl}$ patients at 2 years follow-up was additionally investigated. Results: Compared to controls, $\mathrm{MCl}$-patients had lower $\mathrm{GM}$ volumes in the parahippocampus ( $E S=1.34)$, posterior cingulum $(E S=1.29)$ and isthmus $(E S=1.03$ ) and AD-patients showed lower baseline volumes in the hippocampus ( $E S=1.48$ ), parahippocampus $(E S=1.33)$ and isthmus cingulate $(E S=0.72)$. All investigated ROIs showed group differences in WM integrity with highest ES found for parahippocampal (ES>3.0) and hippocampal diffusivity (ES>2.5), followed by posterior cingulum $(E S \pm 2.5)$, isthmus $(E S \pm 2.0)$ and genu ( $E S \pm 1.8)$. No differences were found between MCI/AD. Smaller hippocampal volumes in the patient group $(\mathrm{MCl}+\mathrm{AD})$ were associated with higher hippocampal diffusivity. Baseline axial and mean diffusivity values in the genu were able to discriminate $\mathrm{MCl}$-stable and $\mathrm{MCl}$ decline.

Conclusions: DTI metrics are more sensitive for group differences than atrophy measurements. Our results suggest that diffusion changes precede and occur independent of GM atrophy. DTI metrics in the genu are able to predict cognitive decline in $\mathrm{MCl}$. 


\section{INTRODUCTION}

Medial temporal lobe (MTL) atrophy as assessed on structural MRI has proven to be an effective clinical aid in the early diagnosis of AD [1]. MTL atrophy is however not entirely specific for $A D$ since it can also be found in other forms of dementia [2]. It is furthermore known that $A D$ pathology is not restricted to the MTL, also posterior parietal and prefrontal regions are involved $[3,4]$. Apart from GM atrophy, it has been shown that white matter (WM) integrity is decreased in subjects with $\mathrm{MCl}$ and $A D[5]$, suggesting a potential diagnostic value on a microstructural level that may help to improve the diagnostic accuracy of volumetric AD biomarkers. The integrity of WM can be assessed using diffusion tensor imaging (DTI) which measures the diffusion of water molecules in neural tissue [6]. In general, WM integrity of the following regions seems to be affected: hippocampal gyrus, temporal white matter, genu and splenium of the corpus callosum and posterior cingulum [6-9].

The underlying mechanism responsible for these WM changes is not yet known. It is presumed that there is a local variation in cell density, oligodendrocytes, myelination and amyloid plaques causing altered diffusion of water molecules in certain brain regions. In the majority of DTI studies of $A D$, the investigation of WM abnormalities was only performed assessing changes in fractional anisotropy (FA) and mean diffusivity (MD). However, contributions to FA and MD alterations could be due to changes of diffusion either parallel or perpendicular to the principal direction of the tensor. These measurements are referred to as axial (AXD), and radial diffusivity (RAD) respectively. An increase in RAD is thought to signify increased space between fibers (suggesting demyelination or dysmyelination) [10] whereas increases in AXD suggest axonal damage [11]. Alterations in diffusion metrics which reflect the integrity of WM tracts not only may give important cues to elucidate the pathophysiological cascade, but could also have diagnostic potential and thus possibly serve as an early marker for AD.

The aim of this study is to investigate the pattern of gray and white matter alterations in $\mathrm{MCl}$ and AD-patients in a multicenter setting. To date, no study has compared the diagnostic value of both volumetry and DTI-markers in a multicenter setting in both $\mathrm{MCl}$ and $\mathrm{AD}$-patients [12]. Multicenter study designs are important since they show greater generalizability of findings than single-center studies. First, we 
explored the ability of automated GM atrophy measurements and DTI metrics to discriminate between healthy controls (Con), subjects with $\mathrm{MCl}$, and subjects with $A D$ in 6 regions of interest (ROI). These ROls were selected based on their pivotal role in $A D$ and consisted of the hippocampus, parahippocampal gyrus, posterior cingulum, isthmus of the cingulum and genu and splenium of the corpus callosum. Effect sizes (ES) were calculated in order to quantify and compare the potential diagnostic value of both DTI and atrophy measurements. In the second place we investigated associations between the degree of GM loss and WM integrity in the selected ROls. Third, we investigated the value of these markers for predicting cognitive decline at 2 years follow-up in $\mathrm{MCl}$ patients.

\section{METHODS}

\section{Subjects}

Healthy controls were recruited by means of an advertisement in local newspapers. An extensive neuropsychological test battery was administered and subjects were included when their performance did not deviate from normal on the Verbal Learning Test $[13,14]$. Patients with $M C l$ and $A D$ were selected from LeARN, a multicenter study investigating the clinical value of novel biomarkers for AD. Four academic memory clinics (Leiden University Medical Centre, Maastricht University Medical Centre, Radboud University Nijmegen Medical Centre and VU University Medical Centre) specialized in the diagnosis and treatment of memory disorders participated in the study. For the present study, participants were selected from 3 of the 4 participating centers where diagnosis based on expert panel judgments was available. Inclusion and exclusion criteria are described in a previous paper [15]. The MCl-group consisted of both amnestic and non-amnestic subjects, in order to obtain a representative sample of subjects visiting a memory clinic. An overview of clinical and biomarker characteristics of the AD-group can be found in Appendix 1.

\section{Clinical and cognitive assesment}

Baseline clinical assessment

All participants underwent a standard diagnostic workup, including clinical history taking, medical and neurological examination, clinical chemistry, functional evaluation using the Clinical Dementia Rating scale (CDR) [16], rating scales for depression and neuropsychiatric symptoms, a neuropsychological test battery and neuroimaging. Cerebrospinal fluid (CSF) was collected for a subsample of the total 
population. The MMSE [17] was administered as a general cognitive screening instrument. Subjects were invited annually for follow-up assessment during 2 years, including questionnaires, MMSE and cognitive tests. Diagnosis was made by an expert panel which consisted of a neurologist, a psychiatrist and a geriatrician with at least 5 years of clinical experience. An adjusted Delphi method was applied starting with the assessment of each case by each expert individually, followed by solving all diagnostic discrepancies during a group discussion meeting. Outcome measure was decline (both on a cognitive and/or functional level), which was again determined by the three clinical experts. They were asked to individually judge observed decline by answering the question "In your opinion, what was the course of cognitive or daily functioning during the 2-year follow-up?". The study was approved by the Medical Ethics Committee of each participating center and written informed consent was obtained from all participants and from the primary caregiver of the AD patients in accordance with the committee's guidelines and with the Declaration of Helsinki [18].

\section{Neuropsychological examination}

A neuropsychological test battery was performed to evaluate performance in several cognitive domains. An overview of both patient and informal caregiver assessments at baseline and follow-up is presented in a previous publication [15].

\section{MRI acquisition and imaging analysis}

\section{Scan protocol}

At each site, patients were scanned according to the routine MRI-protocol. Scanners and protocols at different sites varied but all scanning was performed at 3.0 Tesla (See AppendixS1 for a detailed description of the scan protocols). Before the start of the study, a dummy procedure was carried out in order to optimize the homogeneity of scan quality in each center. All scans included a three-dimensional T1 weighted gradient echo (3DT1) sequence and diffusion weighted images (DTI) (See Tables S1 and S2 for a detailed description of the scan protocols).

\section{Preprocessing of diffusion data}

The DTI data were processed with the freely available MR diffusion toolbox ExploreDTI version 4.8.3 (www.exploredti.com) [19]. Briefly, in a sequence of preprocessing steps the diffusion-weighted images were corrected for subject motion 
and eddy current induced distortions, incorporating the B-matrix rotation to preserve the diffusion gradient orientation information correctly [20]. Correction for echo-planar imaging (EPI) distortion was performed using T1 data as reference [21]. The diffusion tensor was estimated with "RESTORE", a robust non-linear leastsquares method [22]. From this diffusion tensor, the DTI metrics FA, MD, RAD and AXD were derived.

\section{FreeSurfer automated volumetry}

Cortical reconstruction and volumetric segmentation was performed with FreeSurfer version 5.1 .0 (http://surfer.nmr.mgh.harvard.edu). The precise technical details of these procedures are described elsewhere [23, 24]. In short, each dataset is corrected for intensity non-uniformities, skull-stripped, and then segmented to identify the boundaries between GM and WM and to create a surface representation of the cortical WM. Finally, the cerebral cortex is parcellated into units based on its gyral and sulcal structure [25]. According to probabilistic information estimated from a reference atlas, a neuroanatomical label is assigned to each vertex of the surface model and the corresponding measures (i.e., volume) are calculated for each section. All procedures with FreeSurfer are conducted in native space. The following GM regions have been chosen for further analysis because they all share an early vulnerability to AD pathology: hippocampus, parahippocampal gyrus, posterior and isthmus cingulate (See supplemental figure S1).

Template based ROI-analyses of DTI metrics

Regional WM integrity was assessed using the DTI metrics FA, MD, RAD and AXD. ROIs were mainly adopted from the cortical parcellation analysis, now focussing on WM regions just below the cortical mantle. Because of the importance of diffusivity changes in callosal regions in early $A D$, we additionally investigated diffusion abnormalities in the genu and splenium of the corpus callosum. Bilateral ROIs were created based on a template (called 'Bert') provided by FreeSurfer. Because the results of ExploreDTI were in native T1 space, the FA, MD, AXD, and RAD volumes were mapped to this participant on the basis of the registration of each T1 to this participant using FSL's flirt and fnirt from the FSL toolbox (version 5.0.4, see http:// fsl.fmrib.ox.ac.uk/fsl/fslwiki [26]). Within the selected WM ROIs the average of each DTI metrics was computed. 


\section{CSF analyses}

CSF was obtained by lumbar puncture between the L3/L4 or L4/L5 intervertebral space, and collected and aliquoted into polypropylene tubes. CSF samples were stored at $-80^{\circ} \mathrm{C}$ at each center and samples of Maastricht and Nijmegen were transported on dry ice to Amsterdam for analysis after up to 2.5 years. The commercially available INNOTEST enzyme-linked immunosorbent assay was used (ELISAs; Innogenetics, Ghent, Belgium) to quantify CSF Ab1-42, performed by experienced laboratory technicians.

\section{Statistical analysis}

Analysis was performed with the Statistical Package for Social Sciences (SPSS version 20). Between-group differences on clinical, demographic and cognitive characteristics were analyzed using the independent sample t-test for continuous and $\chi 2$ for categorical variables (significant values set at $p<0.05$ ). Univariate analysis of covariance (ANCOVA) was used to test the discriminative ability of CSF markers, gray matter volume and average DTI metrics. Group differences were investigated in the splenium and genu of the corpus callosum, left and right hippocampus, parahipocampal gyrus, posterior and isthmus cingulum. Gender, demeaned age and demeaned center code were included as covariates to account for the potential effects of age, gender and multicenter settings on group differences. Effect sizes were calculated using Hedge's $g$, which is the difference between the group means divided by the pooled standard deviation. Effect sizes between 0.2 and 0.5 were classified as small, between 0.5 and 0.8 as medium, and over 0.8 as large [27]. In order to investigate the relation between GM and WM tissue abnormalities, correlation analysis (one-tailed) were conducted within the patient group ( $\mathrm{MCl}$ and $A D$ ). To detect whether WM abnormalities were independent of GM abnormalities, GM volumes were entered as a covariate in one of the statistical models. When volumetric data were analysed, intracranial volume (ICV) was added as a control variable. In order to detect which markers (CSF, atrophy or DTI) at baseline were able to predict decline at follow-up, we investigated which markers differed between the groups of $\mathrm{MCl}$-decline and $\mathrm{MCl}$-stable.

\section{Correction for intracranial volume (ICV)}

To account for age-related cortical shrinkage we measured the intracranial volumes 
(ICV) with the FSL Brain Extraction Tool [28].

\section{Correction for multiple comparisons}

To control for Type I errors, we performed the false discovery rate (FDR) controlling procedure in all cases, a correction for multiple comparisons [29]. This method differs from the classical Bonferroni approach in that it is less stringent and as such also reduces the increased probability of making Type II errors.

\section{RESULTS}

\section{Subject characteristics}

$\mathrm{MCl}$ and $\mathrm{AD}$-patients differed from healthy controls in terms of age (Con vs $\mathrm{MCl}$ : $p<.01$, Con vs AD: $p<.001)$, gender $(p<.001)$, MMSE $(p<.001)$, delayed recall $(p<.001)$ and MTA-score (Con vs $\mathrm{MCl}: p<.05$, Con vs AD: $p<.001$ ) (Table 1). AD-patients showed lower MMSE $(p<.001)$ and lower delayed recall scores $(p<.05)$ compared to $\mathrm{MCl}$-patients. CSF values of t-tau $(p<.05)$ were higher in AD-patients compared to $\mathrm{MCl}$. Values of CSF $A \beta 1-42$ and the degree of MTL atrophy as assessed by a visual rating scale (MTA-score) did not differ between both patient groups (Table 1 ).

\section{Patterns of gray matter atrophy}

Relative to controls, $\mathrm{MCl}$-patients had significantly lower GM volumes in the left and right parahippocampus $(p<.001)$, right posterior cingulum $(p<.05)$, and left $(p<.01)$ and right isthmus of the cingulum $(p<.001)$ (Table 2$)$. After correction for multiple comparisons, only left $(p<.01)$ and right parahippocampal gyrus volume $(p<.01)$ significantly differed between Con and AD. No significant GM atrophy differences were found between $\mathrm{MCl}$ and $\mathrm{AD}$-patients in the investigated regions (Table 2).

\section{Loss of WM integrity}

$\mathrm{MCl}$-patients showed lower FA values in the bilateral isthmus and posterior cingulate, parahippocampal gyrus (all $p<.001$ ), hippocampus (left: $p=.013$, right: $p=.002)$ and genu $(p<.001)$ and splenium $(p<.05)$ of the corpus callosum compared to healthy controls. Radial and mean diffusivity of the isthmus, parahippocampal gyrus, hippocampus and genu were higher in $\mathrm{MCl}$-patients compared to controls (all $p<.001$ ). Diffusivity differences between controls and $\mathrm{MCl}$ were also found in the splenium ( $p=.003$ for MD and $p=.004$ for RAD). Values of axial diffusivity were increased in $\mathrm{MCl}$-patients relative to controls in the posterior and isthmus cingulum, 
parahippocampal gyrus, genu (all $p<.001)$, splenium $(p=.028)$ and hippocampus (left: $p=.01$, right: $p<.001$ ) (Table 3). Patients with AD showed lower FA values in the bilateral isthmus (left: $p<.001$, right: $p<.01)$, posterior cingulum $(p<.001)$, parahippocampal gyrus $(p<.001)$, left hippocampus $(p=.036)$, and genu $(p<.001)$ of the corpus callosum compared to healthy controls. Axial, radial and mean diffusivity of the genu, isthmus, posterior cingulum, parahippocampal gyrus, and hippocampus were higher in AD-patients compared to controls (all $p<.001$ ). Mean diffusivity of the splenium ( $p=.035)$ was higher in AD-patients compared to controls. (Table 3). Similar to the GM atrophy results, no DTI metrics significantly differed between $\mathrm{MCl}$ and AD-patients.

Table 1. Subject characteristics.

\begin{tabular}{|c|c|c|c|}
\hline & Controls & $\mathrm{MCl}$ & $A D$ \\
\hline $\mathrm{N}$ & 18 & 57 & 35 \\
\hline Age (years) & $64.6(3.4) \mp 9$ & $68.5(8.5)$ & $71.80(9.3)$ \\
\hline Male (\%) & $100+9$ & 71 & 66 \\
\hline Years education & $5.9(2.3)$ & $6.0(2.8)$ & $5.2(2.7)$ \\
\hline MMSE score & $28.9(1.0)+9$ & $25.8(3.0) 9$ & $22.6(3.2)$ \\
\hline Wordlist delayed recall & $8.6(1.9)+9$ & $3.7(3.0)^{\wedge}$ & $2.3(3.3)$ \\
\hline MTA-score & $1.1(0.9) \diamond \emptyset$ & $2.1(2.8)$ & $2.7(2.0)$ \\
\hline CSF A $\beta 1-42$ & - & $684.3(250.7)$ & $598.6(230.8)$ \\
\hline CSF t-tau (pg/mL) & - & $471.9(285.6)^{\wedge}$ & $644.8(348.6)$ \\
\hline CSF p-tau $(p g / m L)$ & - & $54.6(26.4)$ & $68.2(35.3)$ \\
\hline
\end{tabular}

NOTE: Values are mean (SD). Abbreviations: MMSE $=$ Mini-Mental state examination; MTA $=$ medial temporal lobe atrophy; $\mathrm{A} \beta 1-42=$ beta amyloid $1-42 ; \mathrm{t}$-tau $=$ total tau, $\mathrm{p}$-tau $=$ phosphorylated tau; $\mathrm{MCl}=$ mild cognitive impairment; $\mathrm{AD}=$ Alzheimer's disease.

$\dagger p<0.001$ compared with the $\mathrm{MCl}$ group. $¥ p<0.01$ compared with the $\mathrm{MCl}$ group. $\diamond p<0.05$ compared with the $\mathrm{MCl}$ group. $\uparrow p<0.001$ compared with the $\mathrm{AD}$ group. $\$ p<0.01$ compared with the AD group. $\wedge^{\wedge} p<0.05$ compared with the $A D$ group. 
Table 2. Regional gray matter volumes (in $\mathrm{mm}^{3}$ ) of controls and patients with $\mathrm{MCl}$ or AD.

\begin{tabular}{llll}
\hline & Controls & $\mathrm{MCl}$ & $\mathrm{AD}$ \\
\hline Hippocampus & & & \\
Left & $3833(288)$ & $3460(857)$ & $3147(590)$ \\
Right & $3837(286)$ & $3589(773)$ & $3195(655)$ \\
Parahippocampus & & & \\
Left & $1314(170)+\S$ & $951(342)$ & $957(340)$ \\
Right & $1357(295)+\S$ & $965(305)$ & $978(383)$ \\
Posterior cingulate & & & $3835(541)$ \\
Left & $4465(344)$ & $3795(652)$ & $3512(592)$ \\
Right & $4106(427) \diamond$ & $3644(581)$ & \\
Isthmus cingulate & & & $3485(550)$ \\
Left & $3872(521) \ddagger$ & $3320(552)$ & $3201(562)$ \\
Right & $3558(436) \dagger$ & $2982(494)$ &
\end{tabular}

NOTE: All analyses stated in the table are corrected for ICV, age, gender and center. Values are mean (SD). Abbreviations: $\mathrm{MCl}=$ mild cognitive impairment; $A D=$ Alzheimer's disease. Results are corrected for multiple comparisons [29]. $\dagger p<0.001$ compared with the $\mathrm{MCl}$ group, $¥ p<0.01$ compared with the $\mathrm{MCl}$ group. $\diamond p<0.05$ compared with the $\mathrm{MCl}$ group, $\S p<0.01$ compared with the AD group.

\section{Quantification and comparison of effect sizes of volumetry and DTI-metrics} In order to quantify and compare the potential diagnostic value of DTI and atrophy measurements, effect sizes were calculated. Highest ES (>3.0) was found for diffusivity measurements in the parahippocampal gyrus (Con vs $A D$, Con vs $\mathrm{MCl}$ ) and hippocampus (Con vs $\mathrm{MCl}$, Con vs $\mathrm{AD}$ ), followed by diffusivity measurements in the posterior and isthmus cingulate and genu (all Con vs $\mathrm{MCl}$, Con vs $A D$ ). Volumetric measurements showed somewhat lower effect sizes ranging from 0.7 (hippocampus, Con vs MCl) to 1.48 (hippocampus, Con vs AD) (supp Table S3). 


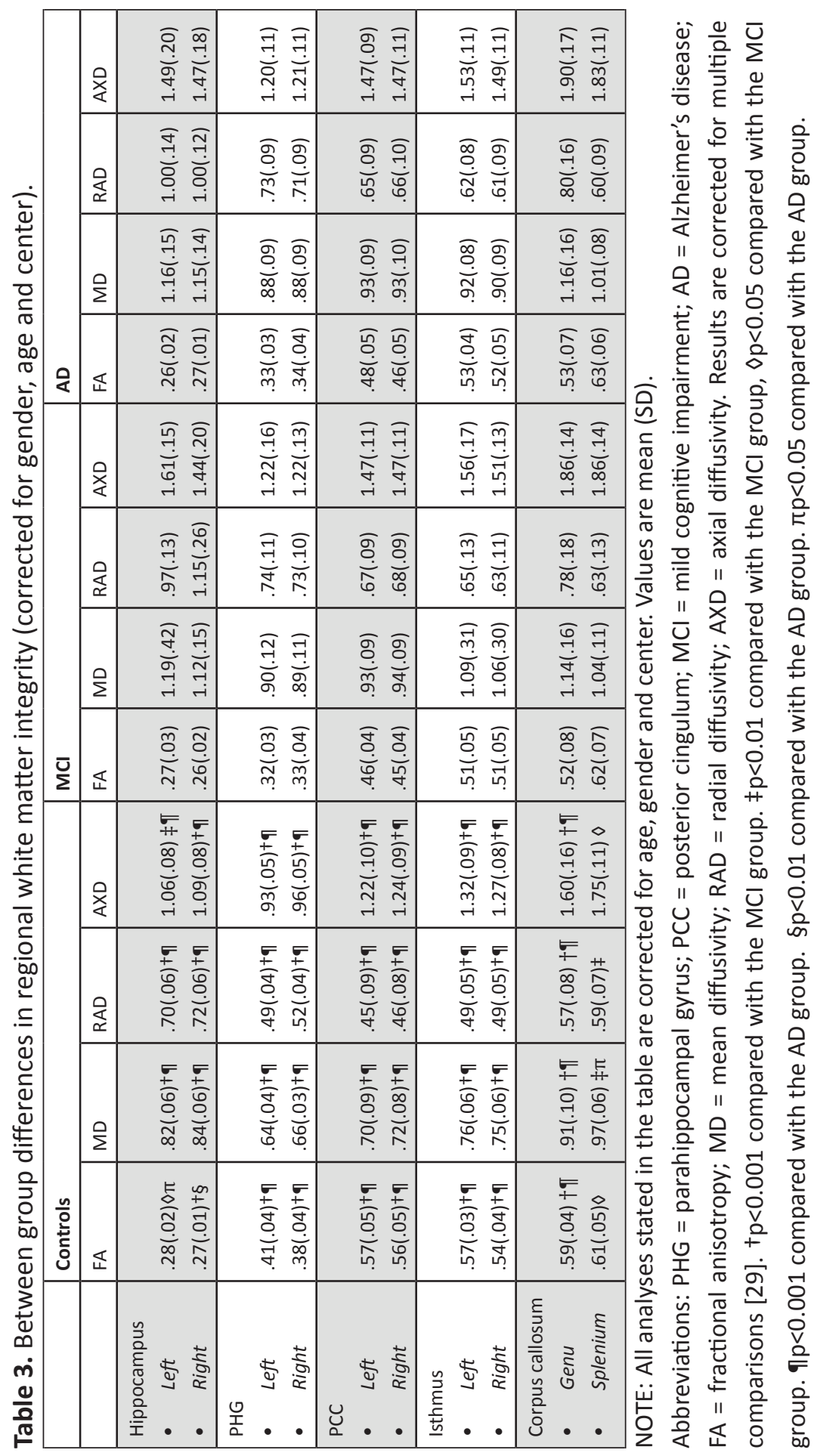




\section{Associations between gray and white matter abnormalities}

In the patient group ( $M C I / A D, n=92$ ) was found that smaller hippocampal volumes were associated with higher values of hippocampal diffusivity (MD, RAD, AXD), especially in the left hemisphere (Fig. 1, supplemental Table S4).

Group differences in WM diffusivity did not change when analyses were additionally corrected for GM volumes indicating that WM diffusivity differences were independent of GM atrophy patterns.

\section{$\mathrm{MCl}$ decline vs $\mathrm{MCl}$ stable}

None of the GM volumes of the investigated structures or CSF values differed between $\mathrm{MCl}$-patients showing decline at follow-up compared to $\mathrm{MCl}$-patients that remained stable (Table 4). With respect to diffusivity, values of MD ( $p=.013$ ) and AXD ( $p=.001)$ of the genu were higher in MCl-subjects who clinically declined compared to $\mathrm{MCl}$-subjects without decline (Table 4, Fig. 2). 
Table 4. Differences between $\mathrm{MCl}$-stable and $\mathrm{MCl}$-decline.

\begin{tabular}{|c|c|c|}
\hline & MCl-stable & $\mathrm{MCl}$-decline \\
\hline $\mathrm{N}$ & 26 & 31 \\
\hline Age & $65.2(9.0)^{* *}$ & $71.3(7.0)$ \\
\hline Male (\%) & 65 & 74 \\
\hline Years education & $5.8(3.0)$ & $6.1(2.7)$ \\
\hline Baseline MMSE score & $25.7(2.5)$ & $25.7(3.3)$ \\
\hline $\begin{array}{l}\text { Baseline wordlist delayed } \\
\text { recall }\end{array}$ & $4.4(3.0)$ & $3.2(2.9)$ \\
\hline \multicolumn{3}{|l|}{ Hippocampus (mm³) } \\
\hline Left & $3765(555)$ & $3203(771)$ \\
\hline Right & $3844(674)$ & $3373(796)$ \\
\hline \multicolumn{3}{|l|}{ Parahippocampus (mm³) } \\
\hline Left & $1002(315)$ & $907(361)$ \\
\hline Right & $1010(298)$ & $926(310)$ \\
\hline \multicolumn{3}{|l|}{ Posterior cingulum $\left(\mathrm{mm}^{3}\right)$} \\
\hline Left & 3909 (501) & $3700(751)$ \\
\hline Right & $3841(472)$ & 3477 (619) \\
\hline \multicolumn{3}{|l|}{ Isthmus of the cingulum } \\
\hline Left & $3282(463)$ & $3351(595)$ \\
\hline Right & $3043(486)$ & $2930(501)$ \\
\hline
\end{tabular}

Genu corpus callosum

$F A$

$M D$

$R A D$

$A X D$

MTA-score

CSF A $\beta 1-42$

CSF t-tau

CSF p-tau
$.54(.09)$

$1.07(.14)^{*}$

$.72(.17)$

$1.78(.11)^{* *}$

$1.2(1.6)$

$733.4(283.5)$

403.8 (345.4)

47.1 (30.9)
$.51(.08)$

$1.20(.15)$

$.84(.17)$

$1.92(.15)$

$2.7(3.3)$

$637.5(211.4)$

536.9 (201.9)

NOTE: Volumetric measurements are corrected for intracranial volume. Values are mean (SD). Abbreviations: $\mathrm{MMSE}=$ Mini-Mental state examination; $\mathrm{MTA}=$ medial temporal lobe atrophy; $F A=$ fractional anisotropy; $M D=$ mean diffusivity; $R A D=$ radial diffusivity; $A X D$ $=$ axial diffusivity; $\mathrm{A} \beta 1-42$ = beta amyloid $1-42 ; \mathrm{t}$-tau = total tau, $\mathrm{p}$-tau = phosphorylated tau; $\mathrm{MCl}=$ mild cognitive impairment; $A D=$ Alzheimer's disease. Results are corrected for multiple comparisons [29]. ${ }^{*} p<.05, * * p<.01$ for differences between groups. Analyses were corrected for age and center (no gender differences were present). 

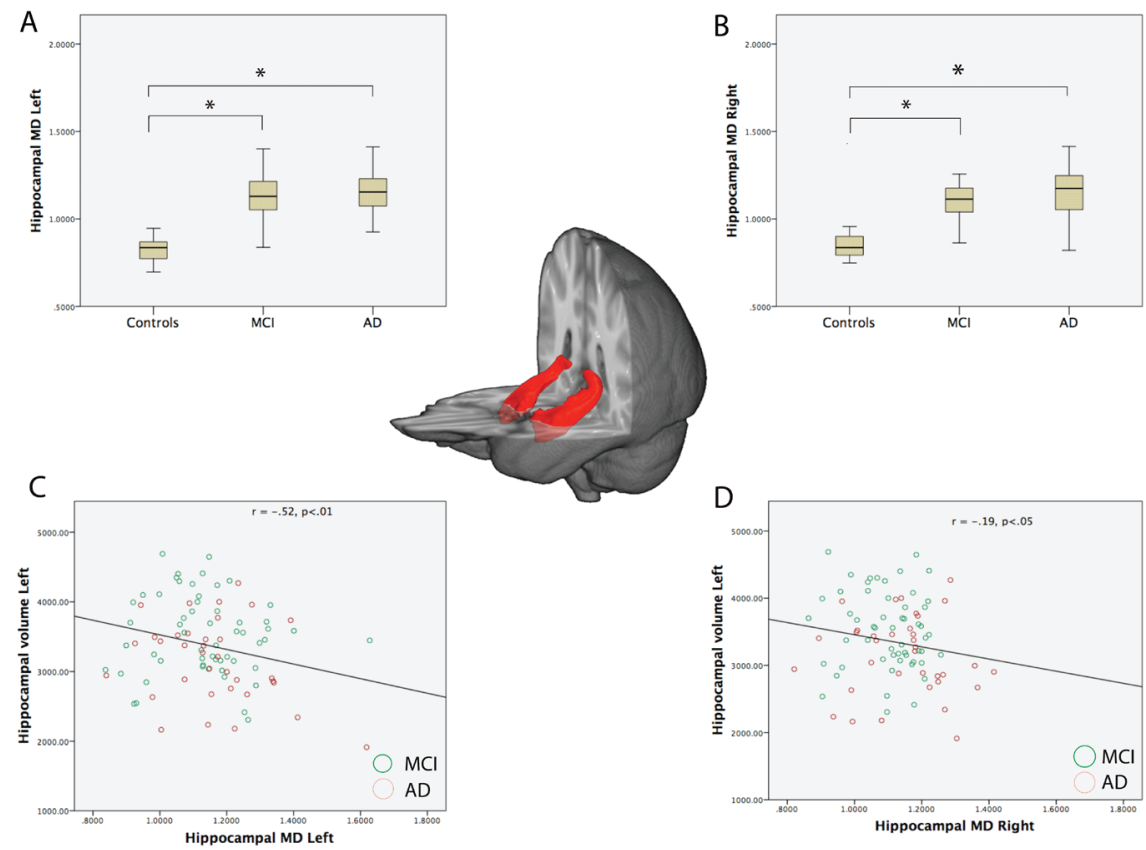

Fig. 1. Associations between volumetric and mean diffusivity values in hippocampal regions. (A, B) Group differences between controls, $M C l$ and $A D$ for left $(A)$ and right (B) hippocampal mean diffusivity (*all $p<.001$ ), (C) Associations between diffusivity in the left hippocampus ( $r=-.52 ; p=<.01)$ and (D) Associations between left hippocampal volume and mean diffusivity in the right hippocampus ( $r=-.19$; $p<.05)$. The bilateral hippocampus was the only structure, among those considered in the present study, where associations were found between GM atrophy and WM integrity. NOTE: $\mathrm{MCl}=$ mild cognitive impairment; $A D$ = Alzheimer's disease; $M D=$ mean diffusivity. 

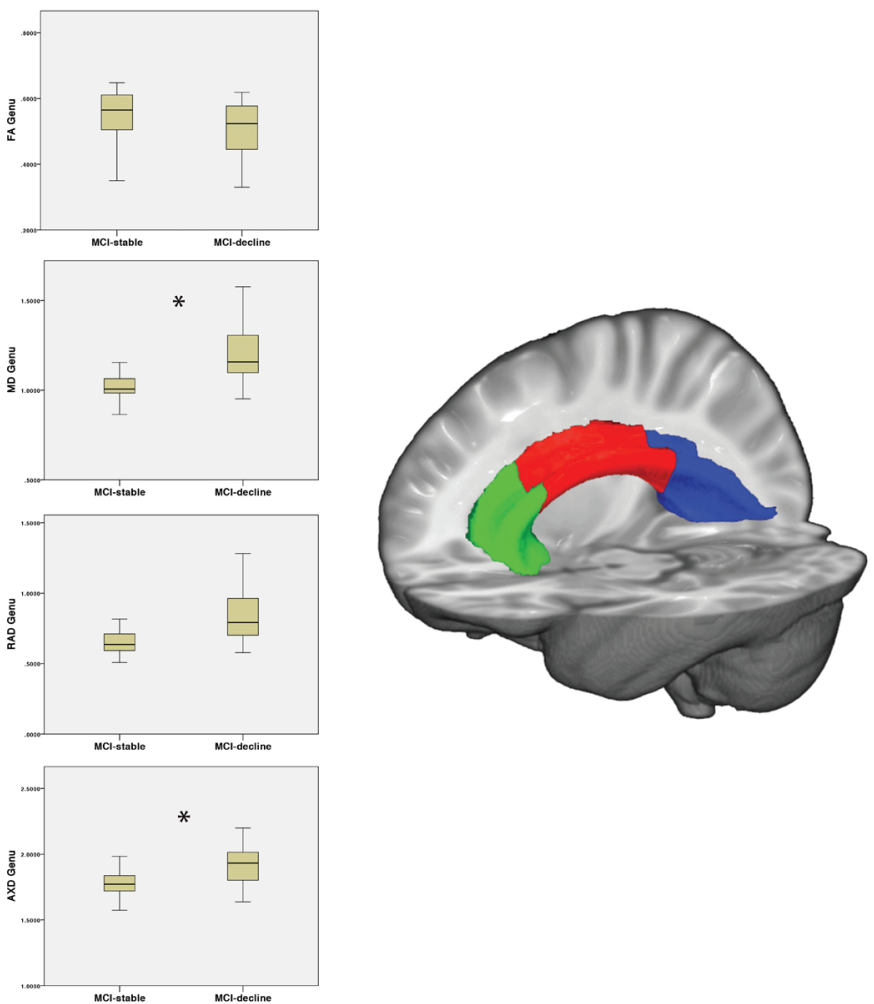
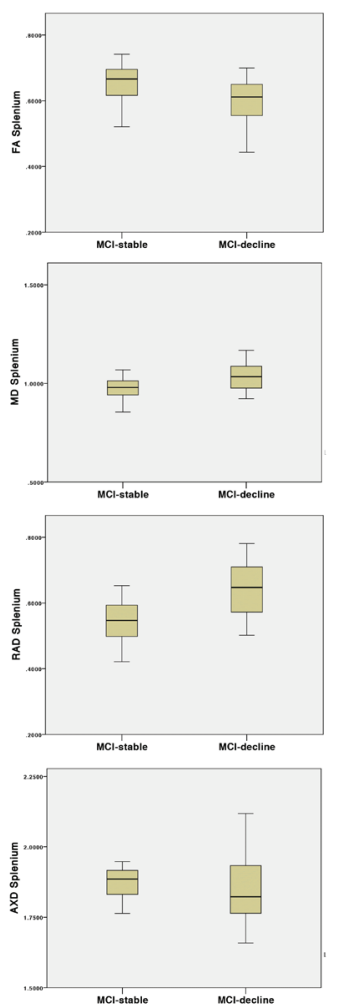

Fig. 2. Diffusivity differences in anterior and posterior parts of the corpus callosum in $\mathbf{~ M C l}$-stable vs $\mathbf{~ M C l}$-decline (2 years follow-up). The anterior part of the corpus callosum (CC) (genu) is depicted in green and the posterior part of the CC is depicted in blue (splenium.) *Differences between $\mathrm{MCl}$-stable and $\mathrm{MCl}$-decline were found in the genu: mean and axial diffusivity metrics (MD: $p=.013, \operatorname{AXD} p=.04$ ). No differences in anisotropy (FA) or radial diffusivity (RAD) were found between $\mathrm{MCl}$ stable and $\mathrm{MCl}$-decline. NOTE: $\mathrm{MCl}=$ mild cognitive impairment; $\mathrm{FA}=$ fractional anisotropy; $M D=$ mean diffusivity; $R A D=$ radial diffusivity; $A X D=$ axial diffusivity. 


\section{DISCUSSION}

The present study applied a combined volumetric MRI and DTI method on selected regions of interest to obtain complementary information on both $\mathrm{GM}$ atrophy and microstructural WM alterations in brain tissue of $\mathrm{MCl}$ and $\mathrm{AD}$-patients in a multicenter setting. The concomitant measurement of both GM volume and WM diffusion measures allowed us to detect the brain tissue effects of underlying ADpathology. Together with the expert panel diagnosis, the novelty in this study was the investigation of the potential of DTI to discriminate between progressive and stable $\mathrm{MCl}$-patients in relation to volumetry, CSF and neuropsychological data.

It was found that diffusivity measurements are more sensitive for group differences (higher ES) than atrophy measurements. DTI metrics are furthermore not significantly influenced by the effects of ageing, gender and center in apriori selected ROIs. Group differences in WM diffusivity did not change when analyses were additionally corrected for GM volumes indicating that WM diffusivity differences were independent of GM atrophy patterns. Baseline axial and mean diffusivity values in the genu were able to discriminate $\mathrm{MCl}$-stable and $\mathrm{MCl}$-decline.

Previous findings showed a widespread pattern of $G M$ atrophy in $A D$ and a more restricted $\mathrm{GM}$ atrophy pattern in $\mathrm{MCl}$ patients [30]. In our study, a similar pattern of $\mathrm{GM}$ differences was found in the bilateral parahippocampal gyrus (Con/MCl and $\mathrm{Con} / \mathrm{AD})$, isthmus of the cingulum $(\mathrm{Con} / \mathrm{MCl})$ and right posterior cingulum (Con/MCl). Other structures were more susceptible to ageing (hippocampus) effects or did not survive the correction for multiple comparisons (hippocampus, left posterior cingulum, isthmus of the cingulum (Con/AD)). Group differences in diffusivity were evident in all the investigated structures. Highest ES was found for the parahippocampal gyrus, followed by the hippocampus, posterior and isthmus cingulate, and genu (Con/AD). For the discrimination between Con/ $\mathrm{MCl}$, ES was highest for the parahippocampal gyrus, followed by the hippocampus and posterior cingulate. In line with previous studies we found that changes in absolute diffusivity (MD, RAD and AXD) were more sensitive for group differences than changes in anisotropy (FA) [31]. This could be explained by the fact that if both $A X D$ and RAD are increased in $A D, M D$ is increased and therefore FA can remain relatively unperturbed [32]. Important to note is that DTI group differences were 
less influenced by the effects of ageing, gender and scanner differences than GM atrophy differences.

\section{Predictors of decline}

Only baseline diffusivity (MD and AXD) in the genu of the corpus callosum differed between $\mathrm{MCl}$-decline and $\mathrm{MCl}$-stable. These differences were furthermore independent of age, gender or site differences. No demographic, CSF or volumetric measurements were able to predict cognitive decline after 2 years in the group of $\mathrm{MCl}$ patients. Several studies found an early involvement of the genu in early and preclinical stages of $A D[33,34]$. A possible explanation for our findings is that the genu contains the highest density of small-diameter fibers, which are known to be most susceptible to neurodegeneration [35]. As a result, WM damage in the genu becomes more evident with disease progression, and diffusion metrics in this region thus allow discrimination between decliners and non-decliners. Secondly, with disease progression, inter-hemispheric connections of prefrontal regions become abnormal, a process in which the genu is known to play a central role [36]. We indeed found differences between $\mathrm{MCl}$-stable and $\mathrm{MCl}$-decline on the verbal fluency test, a test known to rely on prefrontal regions during active retrieval of semantic information $(p=.02)$. Other authors however state that the splenium of the $C C$ is the site of maximum damage in early AD [32]. Despite the fact that diffusivity in the splenium differed between both patient groups and controls, the splenium did not show any predictive value for decline in the group of $\mathrm{MCl}$-subjects.

\section{Loss of WM integrity is independent of GM atrophy}

The associations between the degree of GM atrophy and elevated values of absolute diffusivity (MD, RAD, AXD) in the bilateral hippocampus are in line with previous studies [37] and suggest the presence of Wallerian degeneration of connecting fibers, secondary to GM atrophy in this region [38]. However, our results did not change when analyses were additionally corrected for GM volume, suggesting that WM diffusivity differences were independent of GM atrophy patterns and that Wallerian degeneration is not the primary mechanism of WM change in AD. The lack of a significant correlation between diffusivity and volumetric measurements in regions outside the hippocampus strengthens the idea that DTI and MR-based volumetry measures represent different aspects of underlying pathology [39]. These findings are in line with previous work $[33,40]$ and neuropathological evidence indicating 
that WM changes in $A D$ are not solely due to Wallerian degeneration [41].

\section{Similarities between $\mathrm{MCl}$ and AD-patients}

$\mathrm{MCl}$ and $A D$ patients differed with respect to MMSE and delayed recall scores $(M C l>A D)$ in the absence of $G M$ or $W M$ differences. Values of diffusivity (MD, RAD, $A X D$ ) in some ROls were even lower in $A D$ compared to $\mathrm{MCl}$ patients. These inconsistencies are likely to be due to differences in the clinical stage of the recruited patients. Since $\mathrm{MCl}$ patients represent a heterogeneous group of subjects, their profile of GM and WM abnormalities may be similar to that of $A D$ patients, without major consequences in daily life. Another possibility is that the majority of loss of WM integrity occurs at the time of disease manifestation, while further progression to dementia is accompanied by lower rates of WM change. Previous studies found the same pattern of overlap between $\mathrm{MCl}$ and $\mathrm{AD}$ [42-44]. The similarity of in vivo volumetric and diffusivity measures in the $A D$ and $\mathrm{MCl}$ groups, together with a different clinical status, could be explained by the fact that the $A D$ group has less cognitive reserve which could have led to an earlier transition to $A D$ compared to an extended $\mathrm{MCl}$ status in the group with less impaired global and episodic memory impairment. Except for the higher preservation of cognitive functioning, subjects with $\mathrm{MCl}$ did not differ from AD patients with respect to educational level or head size, both direct indicators of cognitive reserve [45]. Other, more standardized measures of cognitive reserve were however unavailable in the present study. The group of $\mathrm{MCl}$-patients in our study consisted of both amnestic and non-amnestic subtypes. The heterogeneous nature of this sample allowed us to investigate decline in a representative sample of subjects visiting a memory clinic. The same pattern of diffusion abnormalities was furthermore found in $\mathrm{MCl}$-decline vs $\mathrm{MCl}$-stable when only amnestic $\mathrm{MCl}(\mathrm{aMCl})$ subjects were included in the statistical analyses $(n=32)$ (data not shown). Diffusivity abnormalities in the genu were again the only predictors of decline in the group of aMCl.

\section{Strengths and Limitations}

It can be considered a strength that for this diagnostic study a memory clinic population was used as we aim to generalize our findings to these subjects who are eligible candidates for clinical trials. As a consequence, however, our findings may not be applicable to other settings, including the general population. The 
use of different MR imaging units and acquisition protocols could have potentially introduced bias. Apart from uniformity during the preprocessing, we additionally corrected for the effect of site differences in all analyses. The present study adopted an automated ROI-analysis based on apriori hypotheses. An advantage is that typical problems associated with voxel-based methods (e.g. alignment) or manual $\mathrm{ROI}$ definition (e.g. subjective, time-consuming) are avoided. A disadvantage of the automated atlas-based ROI approach is that changes are only revealed in preselected areas, lacking whole brain volumetric and diffusion information.

\section{Clinical implications}

Despite the clinical relevance of current DTI-findings in understanding the underlying pathology of $A D$, many of these findings are not yet readily transferrable to clinical practice. The Alzheimer's Disease Neuroimaging Initiative (ADNI) and ADNI-2, did not adopt DTI as a core protocol due to the uncertain long-term test-retest precision [46], questionable relevance to clinical trials, and absence of an established calibration method [47]. These pitfalls should be thoroughly investigated and resolved before DTI can be successfully implemented in everyday clinical practice. In order to implement DTI data as a biomarker for AD, future studies need to establish and validate cut-offs, and standardize automated techniques for use in clinical practice. After overcoming these methodological issues, a quantitative metric of absolute diffusivity (MD, RAD or AXD) could be extracted from posterior parietal (e.g. PCC) or temporal regions (e.g. parahippocampal gyrus or hippocampus) in order to evaluate the presence of neurodegenerative processes (diagnosis). For tracking disease progression, one could measure absolute diffusivity in the anterior part of the corpus callosum (genu) to evaluate which $\mathrm{MCl}$-patients are prone to decline within 2 years (prognosis). The clear boundaries of the corpus callosum make this major WM tract easy to delineate, in comparison to WM portions in medial temporal structures.

\section{CONCLUSION}

The present study demonstrated significant $G M$ and WM differences between $A D$, $\mathrm{MCl}$ and healthy controls in a multicenter setting. Diffusivity measurements are more sensitive for group differences (higher ES) than volumetric measurements and are less influenced by the effects of ageing, gender and center in apriori selected ROls. Our results suggest that diffusion changes precede and also occur independently of 
GM atrophy. DTI metrics in medial temporal, posterior parietal and callosal regions may serve as an early marker for degeneration, even before atrophy is detectable. Diffusivity differences in the genu of the corpus callosum are able to predict decline in a group of $\mathrm{MCl}$-subjects. Translation of the current findings to clinical practice remains to be demonstrated. 


\section{REFERENCES}

1. Clerx L, van Rossum I, Burns L, et al. Measurements of medial temporal lobe atrophy in the prediction of Alzheimer's Disease in subjects with $\mathrm{MCl}$. Neurobiology of Aging 2013.

2. Barber RM, Gholkar AF, Scheltens PM, Ballard CM, McKeith IGM, O'Brien JTD. Medial temporal lobe atrophy on MRI in dementia with Lewy bodies. Neurology 1999;52:1153-1158.

3. Clerx L, Jacobs HIL, Burgmans S, et al. Sensitivity of different MRI-techniques to assess gray matter atrophy patterns in Alzheimer's disease is regionspecific. Current Alzheimer Research 2013;In press.

4. Jacobs HIL, Van Boxtel MPJ, van der Elst W, et al. Increasing the Diagnostic Accuracy of Medial Temporal Lobe Atrophy in Alzheimer's Disease. Journal of Alzheimer's Disease 2011;25:477-490.

5. Bozzali M, Falini A, Franceschi M, et al. White matter damage in Alzheimer's disease assessed in vivo using diffusion tensor magnetic resonance imaging. Journal of Neurology, Neurosurgery \& Psychiatry 2002;72:742-746.

6. Clerx L, Visser PJ, Verhey F, Aalten P. New MRI Markers for Alzheimer's Disease: A Meta-Analysis of Diffusion Tensor Imaging and a Comparison with Medial Temporal Lobe Measurements. Journal of Alzheimer's Disease 2012;29:405429.

7. Chua T, Wen WPD, Chen X, et al. Diffusion Tensor Imaging of the Posterior Cingulate is a Useful Biomarker of Mild Cognitive Impairment. American Journal of Geriatric Psychiatry 2009;17:602-613.

8. Salat DH, Greve DN, Pacheco JL, et al. Regional white matter volume differences in nondemented aging and Alzheimer's disease. Neurolmage 2009;44:1247-1258.

9. Jacobs HIL, Van Boxtel MP, Gronenschild E, et al. Patterns of gray and white matter changes in individuals at risk for Alzheimer's disease. Curr Alzheimer Res 2012;9:1097-1105.

10. Song S-K, Sun S-W, Ju W-K, Lin S-J, Cross AH, Neufeld AH. Diffusion tensor imaging detects and differentiates axon and myelin degeneration in mouse optic nerve after retinal ischemia. Neurolmage 2003;20:1714-1722.

11. Thomas B, Eyssen M, Peeters R, et al. Quantitative diffusion tensor imaging in cerebral palsy due to periventricular white matter injury. Brain 2005;128:2562- 
2577.

12. Teipel SJ WM, Meindl T, Frisoni G, Bokde AL, Fellgiebel A, Filippi M, Hampel $\mathrm{H}$, Klöppel S, Hauenstein K, Ewers M; EDSD study group. Anatomical MRI and DTI in the diagnosis of Alzheimer's disease: a European multicenter study. J Alzheimers Dis. 2012;31:S33-47.

13. Van der Elst W, van Boxtel MP, van Breukelen GJ, Jolles J. Rey's verbal learning test: normative data for 1855 healthy participants aged 24-81 years and the influence of age, sex, education, and mode of presentation. J Int Neuropsychol Soc 2005;11:290-302.

14. Folstein MF, Folstein SE, McHugh PR. "Mini-mental state". A practical method for grading the cognitive state of patients for the clinician. J Psychiatr Res 1975;12:189-198.

15. Handels R, Aalten P, Wolfs C, et al. Diagnostic and economic evaluation of new biomarkers for Alzheimer's disease: the research protocol of a prospective cohort study. BMC Neurology C7 - 72 2012;12:1-8.

16. Morris JC. The Clinical Dementia Rating (CDR): current version and scoring rules. Neurology 1993;43:2412-2414.

17. Folstein MF, Folstein, S.E., \& McHugh, P.R. "Mini-mental state". A practical method for grading the cognitive state of patients for the clinician. Journal of Psychiatric Research 1975;12:189-198.

18. Nylenna M, Riis P. Identification of patients in medical publications: need for informed consent. British Medical Journal 1991;302:1182.

19. Leemans A, Jeurissen B, Sijbers J, Jones DK. ExploreDTI: A Graphical Toolbox for Processing, Analyzing, and Visualizing Diffusion MR Data. Proc. Intl. Soc. Mag. Reson. Med. 2009;17.

20. Leemans A, Jones DK. The B-matrix must be rotated when correcting for subject motion in DTI data. Magn Reson Med 2009;61:1336-1349.

21. Irfanoglu MO, Walker L, Sarlls J, Marenco S, Pierpaoli C. Effects of image distortions originating from susceptibility variations and concomitant fields on diffusion MRI tractography results. Neurolmage 2012;61:275-288.

22. Chang LC, Jones DK, Pierpaoli C. RESTORE: robust estimation of tensors by outlier rejection. Magn Reson Med 2005;53:1088-1095.

23. Dale AM, Fischl B, Sereno MI. Cortical surface-based analysis. I. Segmentation and surface reconstruction. Neurolmage 1999;9:179-194.

24. Fischl B, Sereno MI, Dale AM. Cortical surface-based analysis. II: Inflation,

210 | Chapter 6: A combined volumetry and DTI study 
flattening, and a surface-based coordinate system. Neurolmage 1999;9:195207.

25. Desikan RS, Segonne F, Fischl B, et al. An automated labeling system for subdividing the human cerebral cortex on MRI scans into gyral based regions of interest. Neurolmage 2006;31:968-980.

26. Smith $\mathrm{SM}$, Jenkinson $\mathrm{M}$, Woolrich $\mathrm{MW}$, et al. Advances in functional and structural MR image analysis and implementation as FSL. Neurolmage 2004;23:S208-S219.

27. Cohen J. Statistical Power Analysis for the Behavioral Sciences, Second ed: Lawrence Erlbaum Associates, Hillsdale, New Jersey, USA., 1988.

28. Smith SM. Fast robust automated brain extraction. Human Brain Mapping 2002;17:143-155.

29. Benjamini Y, Hochberg Y. Controlling the False Discovery Rate: A Practical and Powerful Approach to Multiple Testing. Journal of the Royal Statistical Society. Series B (Methodological) 1995;57:289-300.

30. Gili T, Cercignani $M$, Serra L, et al. Regional brain atrophy and functional disconnection across Alzheimer's disease evolution. Journal of Neurology, Neurosurgery \& Psychiatry 2011;82:58-66.

31. Bozzali M, Giulietti G, Basile B, et al. Damage to the cingulum contributes to Alzheimer's disease pathophysiology by deafferentation mechanism. Human Brain Mapping 2012;33:1295-1308.

32. Acosta-Cabronero J, Alley S, Williams GB, Pengas G, Nestor PJ. Diffusion Tensor Metrics as Biomarkers in Alzheimer's Disease. PLoS ONE 2012;7:e49072.

33. Di Paola M, Di lulio F, Cherubini A, et al. When, where, and how the corpus callosum changes in $\mathrm{MCl}$ and $\mathrm{AD}$ : a multimodal MRI study. Neurology 2010;74:1136-1142.

34. Shim YS, Yoon B, Shon Y-M, Ahn K-J, Yang D-W. Difference of the hippocampal and white matter microalterations in $\mathrm{MCl}$ patients according to the severity of subcortical vascular changes: Neuropsychological correlates of diffusion tensor imaging. Clinical Neurology and Neurosurgery 2008;110:552-561.

35. Braak H, Del Tredici K, Schultz C, Braak EVA. Vulnerability of Select Neuronal Types to Alzheimer's Disease. Annals of the New York Academy of Sciences 2000;924:53-61.

36. Zarei M, Johansen-Berg H, Smith S, Ciccarelli O, Thompson AJ, Matthews PM. Functional anatomy of interhemispheric cortical connections in the human 
SUPPLEMENTAL MATERIAL

Supplementary Table S1. T1 scan protocol parameters ${ }^{\mathrm{a}}$

\begin{tabular}{lccccccc}
\hline Center & Mode & TR (ms) & TE (ms) & $\begin{array}{c}\text { Flip angle } \\
\left({ }^{\circ}\right)\end{array}$ & Matrix & Slices & $\begin{array}{c}\text { Voxel size } \\
(\mathrm{mm} 3)\end{array}$ \\
\hline MUMC & 3D FFE & 8.2 & 3.7 & 8 & $240 \times 240$ & 180 & $1 \times 1 \times 1$ \\
VUMC & 3D FFE & 7.8 & 3 & 12 & $256 \times 256$ & 176 & $\begin{array}{c}0.9375 \times \\
0.9375 \times 1\end{array}$ \\
NUMC & 3D FFE & 2300 & 4.7 & 12 & $256 \times 256$ & 192 & $1 \times 1 \times 1$ \\
\hline
\end{tabular}

${ }^{a}$ All acquisitions were made in the sagittal plane

Supplementary Table S2. DTI scan protocol parameters ${ }^{a}$

\begin{tabular}{lcccccccc}
\hline Center & TR (ms) & TE $(\mathrm{ms})$ & Matrix & Slices & $\begin{array}{c}\text { Voxel size } \\
\left(\mathrm{mm}^{3}\right)\end{array}$ & B0 & $\begin{array}{c}\text { Direc- } \\
\text { tions }\end{array}$ & $\begin{array}{c}\mathrm{b} \text { value }(\mathrm{s} / \\
\left.\mathrm{mm}^{2}\right)\end{array}$ \\
\hline MUMC & 8250 & 80 & $128 \times 128$ & 70 & $2 \times 2 \times 2$ & 1 & 61 & 1000 \\
VUMC & 13000 & 94 & $256 \times 256$ & 45 & $1 \times 1 \times 2.4^{\mathrm{c}}$ & 5 & 30 & 1000 \\
NUMC & 13000 & 102 & $128 \times 128$ & 81 & $2 \times 2 \times 2$ & 1 & 30 & 1000 \\
\hline
\end{tabular}

${ }^{\text {a }}$ All acquisitions were made in the axial (transversal) plane

${ }^{\mathrm{b}}$ Number of $\mathrm{B} 0$ acquisitions

${ }^{\mathrm{C}}$ Acquisition was made with a voxel size of $2 \times 2 \times 2.4 \mathrm{~mm}^{3}$ and then interpolated.

Supplementary Table S3. Effect sizes (Hedge's g) of volumetry and DTI metrics.

\begin{tabular}{llccr}
\hline Measure & Group & Hedge's g & \multicolumn{1}{c}{ Cl 95- } & \multicolumn{2}{c}{ Cl 95+ } \\
\hline RAD PHG & Con/AD & -3.45 & -4.32 & -2.57 \\
MD PHG & Con/AD & -3.45 & -4.32 & -2.57 \\
AXD PHG & Con/AD & -3.16 & -4 & -2.32 \\
RAD PHG & Con/MCl & -3.02 & -3.74 & -2.3 \\
MD HC & Con/AD & -2.98 & -3.79 & -2.17 \\
MD PHG & Con/MCl & -2.91 & -3.62 & -2.2 \\
AXD HC & Con/AD & -2.82 & -3.61 & -2.03 \\
RAD HC & Con/AD & -2.79 & -3.57 & -2 \\
RAD HC & Con/MCl & -2.67 & -3.35 & -1.98 \\
AXD PCC & Con/AD & -2.63 & -3.39 & -1.86
\end{tabular}




\begin{tabular}{|c|c|c|c|c|}
\hline \multirow{2}{*}{$\frac{\text { Measure }}{\text { MD PCC }}$} & \multirow{2}{*}{$\frac{\text { Group }}{\text { Con/MCl }}$} & \multirow{2}{*}{$\frac{\text { Cl 95- }}{-2.56}$} & \multicolumn{2}{|c|}{ Cl 95+ } \\
\hline & & & -3.23 & -1.88 \\
\hline MD PCC & Con/AD & -2.56 & -3.31 & -1.8 \\
\hline FA PHG & Con/MCl & 2.55 & 1.87 & 3.22 \\
\hline AXD PHG & Con/MCl & -2.45 & -3.11 & -1.78 \\
\hline RAD PCC & Con/MCl & -2.44 & -3.11 & -1.78 \\
\hline FA PCC & Con/MCl & 2.43 & 1.77 & 3.09 \\
\hline AXD PCC & Con/MCl & -2.38 & -3.03 & -1.72 \\
\hline MD isthmus & Con/AD & -2.26 & -2.98 & -1.54 \\
\hline FA PHG & Con/AD & 2.26 & 1.54 & 2.98 \\
\hline RAD PCC & Con/AD & -2.22 & -2.94 & -1.51 \\
\hline AXD isthmus & Con/AD & -2.09 & -2.79 & -1.39 \\
\hline RAD isthmus & Con/AD & -1.95 & -2.63 & -1.27 \\
\hline MD genu & Con/AD & -1.87 & -2.55 & -1.2 \\
\hline RAD genu & Con/AD & -1.82 & -2.49 & -1.15 \\
\hline AXD genu & Con/AD & -1.82 & -2.49 & -1.15 \\
\hline FA PCC & Con/AD & 1.8 & 1.13 & 2.47 \\
\hline AXD isthmus & Con/MCl & -1.76 & -2.37 & -1.16 \\
\hline AXD genu & Con/MCl & -1.73 & -2.33 & -1.13 \\
\hline MD genu & Con/MCl & -1.72 & -2.32 & -1.12 \\
\hline MD isthmus & Con/MCl & -1.48 & -2.06 & -0.9 \\
\hline HC volume & Con/AD & 1.48 & 0.84 & 2.11 \\
\hline FA isthmus & Con/MCl & 1.46 & 0.88 & 2.04 \\
\hline PCC volume & Con/AD & 1.39 & 0.76 & 2.02 \\
\hline PHG volume & Con/MCl & 1.34 & 0.77 & 1.92 \\
\hline PHG volume & Con/AD & 1.33 & 0.7 & 1.95 \\
\hline PCC volume & Con/MCI & 1.29 & 0.72 & 1.85 \\
\hline MD HC & Con/MCl & -1.23 & -1.8 & -0.67 \\
\hline FA isthmus & Con/AD & 1.13 & 0.52 & 1.74 \\
\hline FA genu & Con/MCl & 1.11 & 0.55 & 1.67 \\
\hline FA genu & Con/AD & 1.05 & 0.45 & 1.66 \\
\hline Isthmus volume & Con/MCI & 1.03 & 0.47 & 1.58 \\
\hline FA HC & Con/AD & 1 & 0.4 & 1.6 \\
\hline AXD splenium & Con/MCl & -0.87 & -1.42 & -0.33 \\
\hline AXD HC & Con/MCl & -0.83 & -1.38 & -0.29 \\
\hline RAD isthmus & Con/MCl & -0.81 & -1.35 & -0.26 \\
\hline MD splenium & Con/MCl & -0.79 & -1.34 & -0.24 \\
\hline
\end{tabular}




\begin{tabular}{llccr}
\hline Measure & Group & Hedge's g & Cl 95- & Cl 95+ \\
\hline AXD splenium & Con/AD & -0.73 & -1.31 & -0.14 \\
Isthmus volume & Con/AD & $\mathbf{0 . 7 2}$ & $\mathbf{0 . 1 4}$ & $\mathbf{1 . 3 1}$ \\
RAD genu & Con/MCl & -0.72 & -1.26 & -0.18 \\
HC volume & Con/MCl & $\mathbf{0 . 6 8}$ & $\mathbf{0 . 1 4}$ & $\mathbf{1 . 2 2}$ \\
MD splenium & Con/AD & -0.57 & -1.14 & 0.01 \\
FA HC & Con/MCl & 0.39 & -0.14 & 0.93 \\
RAD splenium & Con/MCl & -0.38 & -0.92 & 0.15 \\
FA splenium & Con/AD & -0.36 & -0.93 & 0.21 \\
FA splenium & Con/MCl & -0.16 & -0.69 & 0.37 \\
RAD splenium & Con/AD & -0.12 & -0.69 & 0.44 \\
\hline
\end{tabular}

NOTE: Only left side of the brain was taken into account.

$\mathrm{Cl}=$ confidence interval; $\mathrm{RAD}$ = radial diffusivity; $\mathrm{AXD}$ = axial diffusivity; $\mathrm{MD}=$ mean diffusivity; $\mathrm{FA}$ = fractional anisotropy; $\mathrm{PHG}=$ parahippocampal gyrus; $\mathrm{PCC}=$ posterior cingulum; $\mathrm{Con}=$ Controls; $\mathrm{MCl}=$ mild cognitive impairment; $A D=$ Alzheimer's disease.

Supplementary Table S4. Associations between GM atrophy and WM integrity

\begin{tabular}{lcccccc}
\hline Structure & MD L & MD R & RAD L & RAD R & AXD L & AXD R \\
\hline Hippocampus L & $-.52^{* *}$ & $-.19^{*}$ & $-.23^{*}$ & -0.15 & $-.52^{* *}$ & $-.23^{*}$ \\
Hippocampus R & $-.45^{* *}$ & -0.1 & -0.12 & -0.07 & $-.48^{* *}$ & -0.14 \\
\hline
\end{tabular}

NOTE: Differences in anisotropy (FA) were not associated with hippocampal volume. $M D=$ mean diffusivity; $R A D=$ radial diffusivity; $A X D=$ axial diffusivity; $L=$ left, $R=$ right. $* p<.05, * * p<.01$ 

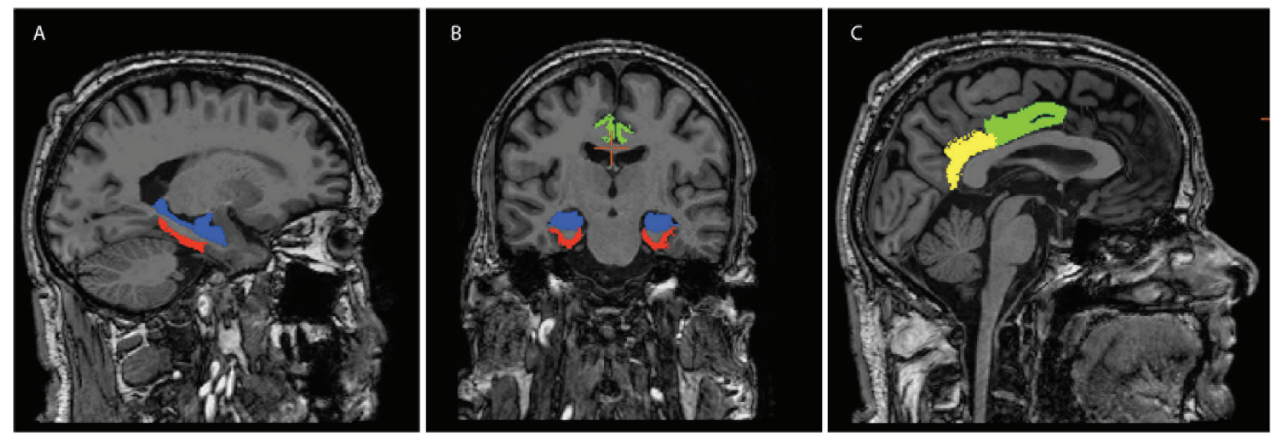

Supplemental Fig. S1. Regions-of-interest selected for the present study. The following structures were selected for further analysis based on their pivotal role in Alzheimer's disease: hippocampus (blue), parahippocampal gyrus (red), posterior cingulum (green), and isthmus of the cingulum (yellow). (A) Sagittal view showing the hippocampus and parahippocampal gyrus, (B) Coronal view showing the posterior cingulum, hippocampus and parahippocampal gyrus and (C) Sagittal view showing the isthmus and posterior part of the cingulum.

Appendix 1. Clinical characteristics of the Alzheimer group

Alzheimer diagnosis was made by an expert panel which had access to clinical, functional and neuropsychological data, together with quantitative MRI information (e.g. vascular pathology (infarcts, microbleeds), degree of medial temporal lobe atrophy (MTA) as measured by the visual rating scale [48]). Since no other biomarker (e.g. CSF) apart from MTA was included in the expert panel diagnosis, some AD-cases a posteriori showed a nonAD-like CSF profile $(n=14)$. We further investigated this group and excluded 2 subjects from the initial group of $37 \mathrm{AD}$-subjects since both patients showed a neuropsychological profile with mainly executive problems together with an MTA-score below cut-off [1], probably due to vascular dementia. From the remaining AD-subjects with a non-AD-like CSF profile $(n=12), 6$ patients were categorized as 'SNAP' [49] showing abeta 1-42 values just above the cut-off ( $>550$ in stead of the suspected $\leq 550$ ) together with elevated values of t-tau $(>375)$ and p-tau (>52) [50]. Of the 6 remaining subjects, 2 showed severe MTA as assessed by the visual rating scale (left+right side, $>6$ ), 3 cases showed severe episodic memory problems matching an Alzheimer profile and 1 patient showed severe memory problems together with highly impaired activity of daily living (ADL). 


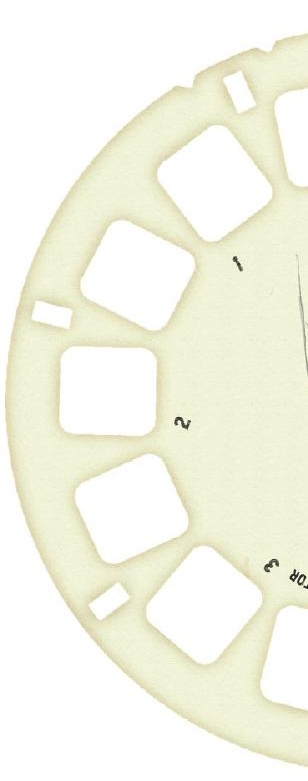




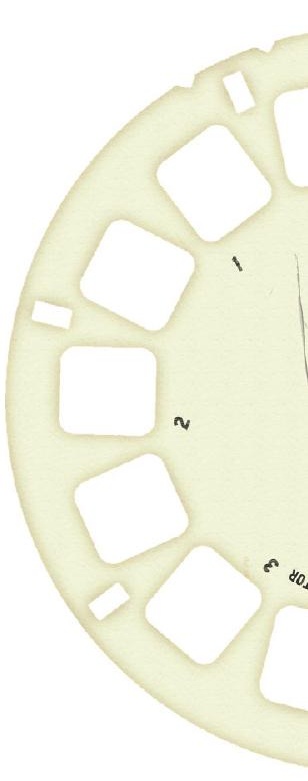




\section{Chapter 7 \\ Can FreeSurfer compete with manual volumetric measurements in Alzheimer's disease?}

$\uparrow$

c)

${ }^{3} \mathrm{royd}^{2}$

Lies Clerx, Ed Gronenschild, Carmen Echávarri, Frans Verhey, Pauline Aalten, Heidi Jacobs 


\begin{abstract}
Alzheimer's disease (AD) results in tremendous structural and functional changes in the brain. The performance of automated brain segmentation techniques may be less precise when applied in AD-patients, possibly leading to false allocations of gray matter, white matter or cerebrospinal fluid. FreeSurfer has been shown to operate as an accurate and reliable instrument to measure cortical thickness and volume of neuroanatomical structures. Considering the principal role of FreeSurfer in the imaging field of $A D$, the present study aims to investigate the robustness of FreeSurfer to capture morphological changes in the brain against varying processing variables in comparison to manual measurements (the gold standard). T1-weighted MRI scan data were used pertaining to a sample of 53 individuals (18 healthy participants, 18 patients with mild cognitive impairment, and 17 patients with mild AD). Data were analyzed with different FreeSurfer versions (v4.3.1, v4.5.0, v5.0.0, v5.1.0), on a custom-built cluster (LINUX) and a Macintosh (UNIX) workstation. Group differences across versions and workstations were most consistent for both the hippocampus and posterior cingulate, regions known to be affected in the earliest stages of the disease. Furthermore, it was found that later versions of FreeSurfer were more sensitive for group differences, and corresponded best with the gold standard. In conclusion, later versions of FreeSurfer were more accurate than earlier versions, especially in medial temporal and posterior parietal regions. This development is very promising for future applications of FreeSurfer in research studies, and encourages the future role of FreeSurfer as a candidate marker in clinical practice.
\end{abstract}




\section{INTRODUCTION}

Considering the increase of the aging population in our society and age being the greatest risk factor for the development of dementia, there is a growing interest in understanding and treating dementia. Currently, Alzheimer's disease (AD) is estimated to affect 35 million patients worldwide (or $0.5 \%$ of the global population) and this number is estimated to increase to 115 million by 2050 [1]. On a brain level, Alzheimer's disease (AD) pathology results in excessive structural and functional damage, secondary to processes such as accumulation of amyloid-beta and tau proteins, neuroinflammation and neuronal death [2]. Structural and functional imaging measurements are currently evaluated for clinical use in predicting or diagnosing $A D[3,4]$. An indispensable part of this effort is the development of a robust method to measure morphological and pathological changes in the brain [5]. Manual volumetric measurements are still regarded gold standard for evaluation of local brain atrophy $[5,6]$, however, clinical settings require diagnostic instruments that are quick, reliable and easy to implement. FreeSurfer (Athinoula A. Martinos Center for Biomedical Imaging, Boston) comprises a popular and freely available set of tools for deriving neuroanatomical volume and cortical thickness measurements by means of automated brain segmentation (http://surfer.nmr.mgh.harvard.edu). At present, more than 80 studies are published using FreeSurfer to investigate structural changes in the brain of (early) AD patients (Source: PubMed, http://www. ncbi.nlm.nih.gov/pubmed/).

Due to the variation in software and hardware environments, both in research and clinical practice, an equally important question related to measuring brain atrophy concerns the power and robustness of automated techniques to capture (small) morphological and pathological changes in the brain against these varying processing conditions. Since the pathological events seen in AD affect the morphology of the brain, it is conceivable that with such fundamental changes in brain structure, minor changes in processing conditions could affect the segmentation process and thus observed group differences. In a previous study we systematically evaluated how the morphometric results derived from FreeSurfer may be affected by the following processing variables: FreeSurfer version (v4.3.1, v4.5.0, and v5.0.0), workstation type (Macintosh and Hewlett-Packard), and Macintosch Operating System version (OSX 10.5 and OSX 10.6) [7]. Results revealed significant differences between 
FreeSurfer version v5.0.0 and the two earlier versions ranging between $8.8 \pm 6.6 \%$ (range 1.3 - 64.0\%) (volume) and $2.8 \pm 1.3 \%$ (1.1 - 7.7\%) (cortical thickness). About twice as small differences were found between either the two workstation types or between OSX 10.5 and OSX 10.6. These differences almost peer with effect sizes reported in neurodegenerative studies. Our previous study investigated changes in healthy young subjects and patients suffering from psychotic disorder [7]. Brain changes in these groups are less pronounced than those found in aging and neurodegenerative diseases. In view of the fact that FreeSurfer is extensively used in studies of ageing, a next step is a validation of FreeSurfer in a neurodegenerative population. The novelty in this study is that a comparison with manual volumetry is included as reference method, which is required in order to validate the accuracy of the segmentation results and thus subsequently detect which abnormalities are due to neurodegeneration and which can be classified as error. Such validation studies require a comparison with manual segmentations or even post- mortem assessments (see [8] for a discussion on the limitations of both reference methods). The accuracy of the cortical thickness measures is more difficult to validate and requires mainly post-mortem (histological) measurements [9]. Only few studies directly compared FreeSurfer with manual volumetric measurements, mainly focusing on the medial temporal lobe in $A D[10,11]$, major depressive disorder [12], and temporal lobe epilepsy $[13,14]$. These studies generally suggest that manual volumetry is slightly superior or equally sensitive to FreeSurfer automated volumetric measurements.

The aim of the present study is to investigate whether the sensitivity of FreeSurfer to detect group differences is consistent over different software versions and operating systems despite tremendous morphological changes typically seen in AD-patients. Stability of group differences across various processing conditions is investigated, and in order to understand which processing condition fits best with the 'reference' group differences, FreeSurfer's group differences are compared with manual volumetry, the gold standard in research practice $[6,15]$. To the best of our knowledge, this is the first study assessing group differences across various processing conditions and validating these findings against gold standard measurements. Based on the literature, six well-established AD signature regions were chosen. Hippocampal atrophy is known to play a major role in the development of AD [16], but is however not specific for AD [17]. Since other medial temporal lobe (e.g. parahippocampal gyrus)[18], prefrontal (e.g. inferior prefrontal 
and orbitofrontal cortex) [19] and posterior parietal regions (e.g. posterior cingulate and precuneus) [20] have shown to be altered during the disease process, these regions are additionally evaluated.

\section{METHODS}

\section{Participants}

Three groups of male participants were included: 18 healthy participants without any subjective memory impairment (CON), 18 patients with amnestic $\mathrm{MCl}(\mathrm{aMCl})$, and 17 patients with mild $A D[18,21]$. Mean age (years \pm sd) of the included individuals was $64.6 \pm 3.4,65.1 \pm 4.5$, and $70.6 \pm 9.1$, respectively. The study was approved by the ethics committee of the Maastricht University Medical Center and all participants gave written informed consent in accordance with the committee's guidelines and with the Declaration of Helsinki [22].

\section{MRI acquisition}

MRI scans were administered with a 3T whole-body MR system release 2.0 (Philips Achieva, Philips Medical Systems, Best, The Netherlands) equipped with an eightelement head coil (SENSE, factor 2). Anatomical T1 images were acquired with a gradient echo sequence with $\mathrm{TR}=8.0 \mathrm{~ms}, \mathrm{TE}=3.7 \mathrm{~ms}, \mathrm{FA}=8^{\circ}$, FOV $=240 \times 240$ $\mathrm{mm} 2$, matrix $=240 \times 240$, number of slices $=180$, voxel size $=1.0 \times 1.0 \times 1.0 \mathrm{~mm}^{3}$.

\section{FreeSurfer}

Automated volumetry

Cortical reconstruction and volumetric segmentation was performed with FreeSurfer, which is freely available (http://surfer.nmr.mgh.harvard.edu). The technical details of these procedures have been described previously (for a recent overview see: [9]) . Briefly, in this approach, brain areas are segmented using a nonlinear template matching [23]. After linearly registering the test image to the template, the algorithm estimates the nonlinear transformation between a given MRI and a probabilistic atlas of the selected brain structure constructed from a cohort of 40 subjects aged 19-87 years using a maximum likelihood criterion [24]. Probabilistic labels are warped back to the individual MRI using the inverse of this transform. The final segmentation is accomplished by maximizing the a posteriori probability in the Bayes formula at each voxel. Voxel-wise probabilistic labels and their predicted 
image intensities serve as the prior term, while the intensity similarity between the target image and the template serves as the likelihood term. In this study, both voxel and tabulated volumes (corrected for partial volume effects) were used. Important to note is that voxel volumes are most suited to obtain a proper comparison with manual volumetry, because of the absence of partial volume correction.

\section{Cortical thickness (CT)}

The Freesurfer CT pipeline has been described and validated in previous publications [25-28]. To summarize, processing involves intensity normalisation, registration to Talairach space, skull stripping, segmentation of WM, tesselation of the WM boundary, smoothing of the tesselated surface and automatic topology correction. The tesselated surface is used as the starting point for a deformable surface algorithm to find the WM and then the pial boundary. For each point on the tesselated WM surface, the $\mathrm{CT}$ is calculated as the average of the distance from the WM surface to the closest point on the pial surface and from that point back to the closest point on the WM surface [26]. The cortex of the brain was automatically subdivided into gyral-based regions of interest (ROIs) [24]. To accomplish this, a registration procedure was used that aligns the cortical folding patterns and probabilistically assigns every point on the cortical surface to one of the $34 \mathrm{ROIs}$. For the purposes of this study, we focused on 6 ROls bilaterally. For each ROI the mean cortical thickness was extracted for subsequent statistical analysis. This technique is referred to as CT-parcellation.

For our second approach, a vertex-wise analysis, the thickness measures were mapped on a spherically inflated surface of each participant's reconstructed brain. This allows visualization across the surface without interference from cortical folding. By means of a combination of linear and non-linear transformations, the spherical cortical folding patterns were aligned to a spherical template provided by FreeSurfer. This technique, called "surface- based intersubject registration" [29], provides an accurate matching of morphologically homologous cortical locations across participants on the basis of each individual's anatomy while minimizing metric distortions. The resulting CT map was smoothed by a circularly symmetric Gaussian filter with a full width half maximum (FWHM) set to $20 \mathrm{~mm}$ in order 1) to compensate for residual misalignments; 2 ) to increase the signal-to-noise ratio; and 3 ) to make the data more normally distributed. This technique is referred to as 
CT-vertex henceforth.

Quality control was performed after each step of the automated FreeSurfer pipeline (volumetry and cortical thickness) to account for possible errors (e.g. misregistrations, outliers).

\section{Manually defined ROIs}

For the manual tracing we used GIANT (General Image Analysis Tools; [30]), a software program which allows tracing of regions of interest (ROI) in a triplanar and rotatable 3D surface-rendered view, and calculation of GM volumes of interest. Boundaries of the selected frontal and temporal structures were set according to criteria described in a previous publication [31]. Boundaries of the posterior cingulate and precuneus cortex were adapted from [32] and [33], respectively. Both raters (LC, CE) were blind to the demographic and cognitive characteristics of the participants. Intra-rater reliability was determined by the Intraclass Correlation Coefficient (ICC) [34]. ICC's for each region can be found in a previous publication [35] (Supplementary Table S1).

Regions of interest (ROI) selected from the Desikan atlas

For the purpose of this study, we used the Desikan atlas and focused on one subcortical ROI and five cortical ROIs bilaterally: the hippocampus, the parahippocampal gyrus (PhG), the inferior prefrontal cortex (IPFC), the orbital frontal cortex (OPFC), the posterior cingulate cortex (PCC), and the precuneus (PC) (see Appendix for more details on which FreeSurfer ROIs [24]) were selected and merged). FreeSurfer ROIs were chosen in accordance with the anatomical borders of the manually defined ROIs: e.g. FreeSurfer's definition of the isthmus was most consistent with our definition of the posterior cingulate cortex as adopted for the manual segmentation and was subsequently used. The posterior cingulate cortex ROI included in the FreeSurfer automated measurement is more rostral compared to our definition.

\section{Intracranial volume}

The ICV was calculated from the inner skull contours produced by the FSL Brain Extraction Tool [36] and visually checked. 


\section{Processing conditions}

\section{Workstations}

Several workstations and corresponding operating systems (OS) were used: an iMac with OSX 10.5, a MacPro with OSX 10.6 (called "iMac2" and "MacPro2" in Gronenschild et al. 2012, respectively), and a custom-built cluster equipped with Intel i7 quad-cores $(3.20 \mathrm{GHz}$ ) running under Scientific Linux 6.2 (called "RadCluster" henceforth). Both Macintosh (Mac) workstations were used in 32 bits mode and the RadCluster in 64 bits mode.

\section{Software versions}

Four versions of FreeSurfer were used: v4.3.1, released on 19 May 2009, version v4.5.0, released on 11 August 2009, version v5.0.0, released on 16 August 2010, and version v5.1.0, released on 24 May 2011. For the Mac workstations these are 32 bits versions, whereas for the RadCluster these are 64 bits versions. An additional remark with respect to v5.1.0 should be made: we used an intermediate version of the processing pipeline in order to resolve issues around the order of the correction for intensity non-uniformity stage and Talairach stage in the pipeline (see also https://surfer.nmr.mgh.harvard.edu/fswiki/TalFailV5.1).

\section{Statistical Analysis}

Group analysis of the segmentation results was performed in two ways. In the first approach, analysis was performed with IBM SPSS version 19 (Chicago, IL, USA) on Macintosh version 19.0. FreeSurfer-based volumes, manual volumes and CTparcellations were compared between the three groups by means of univariate pair-wise ANCOVA with either volume or CT-parcellation as dependent variable, group as independent variable and centered age as covariate. For both volumetric measurements, intracranial volume (ICV) was taken as an additional covariate. To correct for multiple comparisons, we applied the false discovery rate (FDR) controlling procedure [37].

Our second approach was a vertex-wise analysis of CT using FreeSurfer tools. Statistical comparisons between the CT maps were generated by computing a general linear model (GLM) of the CT group differences (corrected for centered age) at each vertex in the cortical mantle, with a statistical threshold set to $p=0.05$. A cluster-wise procedure was performed to correct for multiple comparisons [38]. This 
method utilizes a simulation to get a measure of the distribution of the maximum cluster size under the null hypothesis. Z-maps are synthesized and smoothed using a residual FWHM, and then thresholded at $p=0.05$. Next, areas of maximum clusters are recorded, under these specifications, and the procedure is repeated for 5000 iterations. Once the distributions of the maximum cluster size are obtained, correction for multiple comparisons is performed by finding clusters in the statistical maps using the same threshold as was given in the simulation procedure. For each cluster, the $p$ value is the probability of seeing a maximum cluster of that size, or larger, during the simulation. Clusters remaining in similar areas of significance as in the original cortical thickness maps would indicate that the result is not likely due to chance. For each cluster, maximum, minimum, mean and standard deviation of the $p$-values were extracted.

To quantify the differences of the results of the above vertex-wise analyses across FreeSurfer versions or workstations, the measure of spatial overlap (Dice coefficient, [39]) of the respective clusters was computed. Its range is between 0 (no overlap) and 1 (complete overlap, i.e., exactly similar). It is generally accepted that a value larger than 0.7 indicates a good agreement [40].

\section{RESULTS}

Subject characteristics can be found in Table 1. A complete overview of the comparison CON vs. AD for each selected ROI is illustrated in Fig. 1 (the group comparisons $\mathrm{CON}$ vs. $\mathrm{MCl}$ and $\mathrm{MCl}$ vs. $A D$ are shown in the supplemental material, Figs. S1-S2 respectively). Each cell is color-coded according to its $p$-value after correction for multiple comparisons. In case of CT-vertex we have taken the minimal $p$-value in each ROI. 
Table 1. Subject characteristics

\begin{tabular}{|c|c|c|c|}
\hline & Con & $\mathrm{MCl}$ & $A D$ \\
\hline $\mathrm{N}$ & $18^{\mathrm{a}}$ & $18^{c}$ & 17 \\
\hline Age & $64.56(3.4)$ & $65.11(4.5)$ & 70.59 (9.1) \\
\hline Educational level & $4(1.4)$ & $4(1.8)$ & $4(1.9)$ \\
\hline MMSE score & $28.89(0.9)^{\mathrm{b}, \mathrm{d}}$ & $27.61(2.3)^{f}$ & 21.18 (3.9) \\
\hline 15 WLT learning & $37.50(7.6)$ & $26.06(9.8)$ & $23.47(11.7)$ \\
\hline 15 WLT memory & $8.56(1.9)^{\mathrm{d}, \mathrm{e}}$ & $3.67(2.8)^{c}$ & $1.73(2.4)$ \\
\hline Fluency animals & $23(5.3)$ & $21(5.4)$ & $13.93(4.7)$ \\
\hline $\begin{array}{l}\text { Manual } \\
\text { hippocampus }\end{array}$ & $4656(308) / 4758$ & $4410(482) / 4308$ & $3883(817) / 3807$ \\
\hline volume $\mathrm{L} / \mathrm{R}(\mathrm{mm} 3)$ & -637 & -796 & -876 \\
\hline $\mathrm{ICV}(\mathrm{ml})$ & $1492(100)$ & $1539(121)$ & $1574(125)$ \\
\hline
\end{tabular}

All volumetric measurements are corrected for intracranial volume. Values are mean (sd). MMSE: Mini-Mental state examination;WLT: wordlist; Con: controls; $\mathrm{MCl}$ : mild cognitive impairment; AD: Alzheimer's disease.

${ }^{a} p<0.05$ for difference between $C O N$ and $A D$

${ }^{\mathrm{b}} \mathrm{p}<0.05$ for difference between $\mathrm{CON}$ and $\mathrm{MCl}$

${ }^{c} p<0.05$ for difference between $\mathrm{MCl}$ and $\mathrm{AD}$

${ }^{d} p<0.001$ for difference between CON and AD

e $p<0.001$ for difference between $\mathrm{CON}$ and $\mathrm{MCl}$

${ }^{f} p<0.001$ for difference between $\mathrm{MCl}$ and $\mathrm{AD}$ 


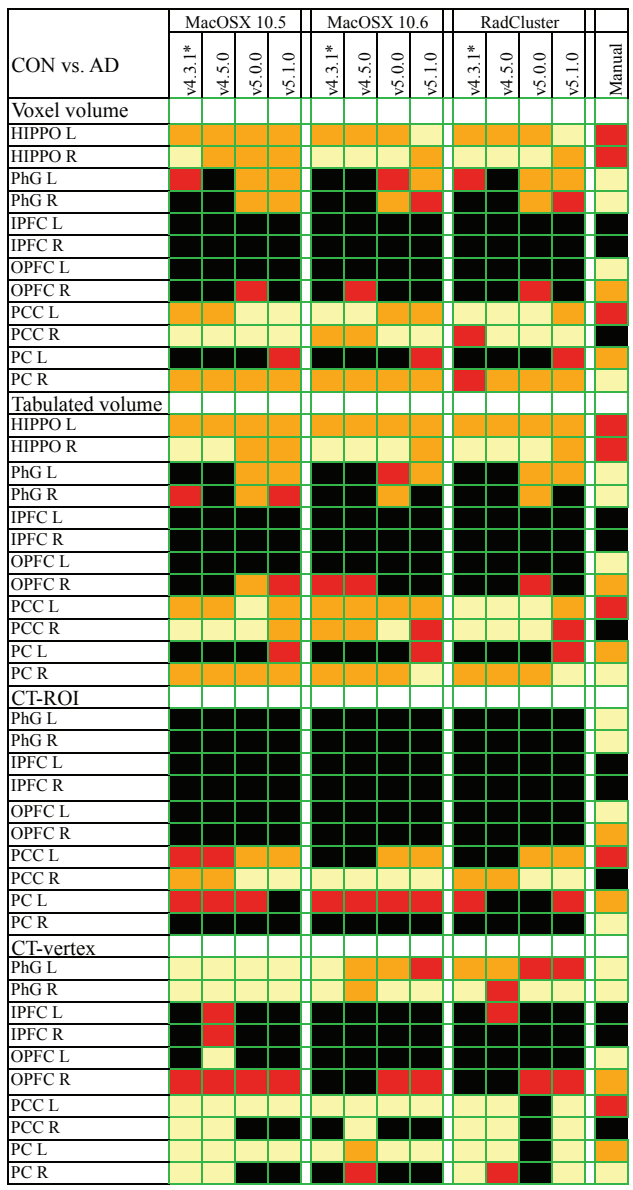

Fig. 1 Overview of statistical significances across FreeSurfer versions and workstations for the comparison of controls versus AD. Each cell is color-coded according to its p-value in four categories, see coding scale at right. The first set of four columns refers to the MacOSX 10.5 workstation, the second set to the MacOSX 10.6 workstation, and the third set to the custom-built cluster workstation (called RadCluster). In each set, the columns present the results for version v4.3.1*, v4.5.0, v5.0.0, and v5.1.0, respectively. Finally, the last column refers to the $p$-values based on the manual outlining. Only the results after correction for multiple comparisons are shown. Four separate FreeSurfer measurements were used: 1) number of voxels within a segmented ROI (called voxel volume); 2) tabulated ROI volume (equal to voxel volume but corrected for partial volume effects); 3) mean cortical thickness (CT) within an ROI (called CT-parcellation-based); 4) vertex-wise CT cluster (called CTvertex). Abbreviations are as follows: $\mathrm{HIPPO}=$ hippocampus; $\mathrm{ERC}=$ entorhinal cortex; $\mathrm{PhG}=$ parahippocampal gyrus; IPFC = inferior prefrontal cortex; OPFC = orbital prefrontal cortex; $\mathrm{PCC}=$ posterior cingulate cortex; $\mathrm{PC}=$ precuneus; $\mathrm{L}=$ left; $\mathrm{R}=$ right. 


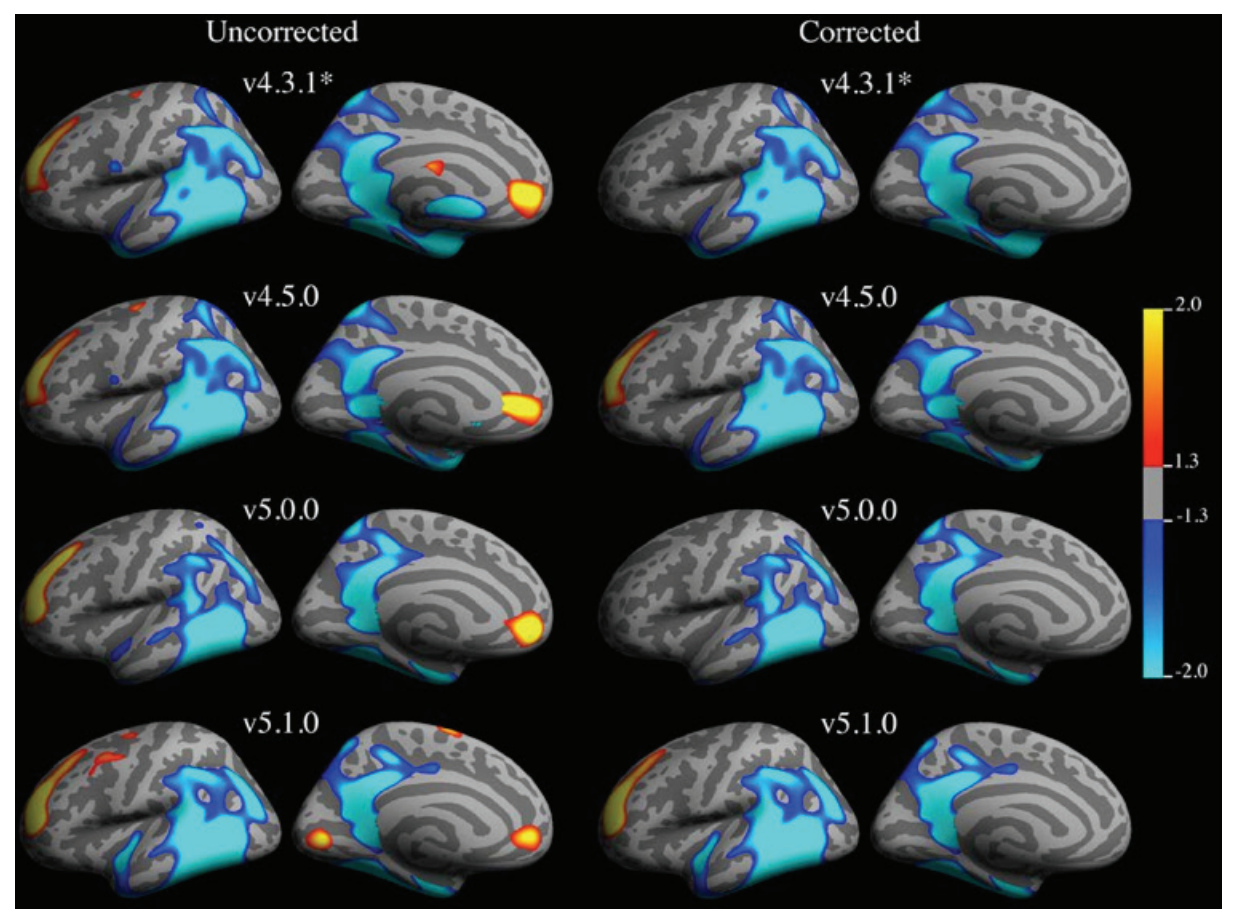

Fig. 2 Significance of cortical thickness differences between controls and ADs obtained from the vertex-wise analysis before and after correction for multiple comparisons (left hemisphere). The color scale at the right depicts the range of $p$-values shown (expressed in 10-x). The colors yellow-red indicate cortical thickening, the color blue indicate cortical thinning. The results for four FreeSurfer versions are displayed, from top to bottom: v4.3.1*, v4.5.0, v5.0.0, and v5.1.0, all obtained on a MacOSX 10.6 workstation.

\section{Robustness of FreeSurfer across workstations and software versions}

Generally, it can be noted that FreeSurfer derives more significant results for later versions in case of volumetric measurements (either voxel or tabulated). In addition, the CT- vertex method produces consistent results through versions as well as workstations, especially for MTL regions. With respect to cross-workstation differences, it can be observed that MacOSX 10.6 is most similar to RadCluster, in particular for v5.1.0. For both voxel and tabulated volumetric measurements, group differences between workstations and software versions were most consistent in hippocampal and posterior cingulate regions. Group differences in the PhG, OPFC R and PC L were only found in FreeSurfer v5.0.0 and/or v5.1.0, across all workstations. The CT-vertex results for MacOSX 10.6 and all FreeSurfer versions are 
illustrated in Fig. 2 for the left hemisphere (comparison CON vs. AD, both corrected and uncorrected results are displayed). Cortical thinning (negative effects, blue colored) was mainly observed in temporal and parietal cortical areas. Cortical thickening (positive effects, red-yellow colored) was apparent in the frontal lobe only for versions v4.5.0 and v5.1.0. For the other versions, these positive clusters did not survive the correction for multiple comparisons. For the comparison CON vs. $\mathrm{MCl}$, no significant clusters were found.

The Dice coefficients for the agreement of the CT surface clusters (positive and negative effects taken together) are summarized in Tables 2 and 3. In most cases the agreement was good to excellent, and better between workstations than between FreeSurfer versions. In order to detail these findings, worst and best agreements for the cross-workstation comparisons are shown in Fig. 3. Vertices depicted in green indicate the presence of the clusters in the results of both workstations. Red and yellow indicate the presence of only one of the respective workstations. The corresponding Dice coefficients were 0.68 and 0.97 , respectively. A complete disagreement was found for the posterior cingulate cortex (see left medial view). Similarly, the worst and best results for the cross-version comparisons are shown in Fig. 4, with corresponding Dice coefficients of 0.58 and 0.94 , respectively. A disagreement was found in the frontal lobe, and posterior cingulate cortex (see left medial view).

Table 2. Cortical thickness surface cluster similarities (Dice coefficients) across workstations

\begin{tabular}{lcc}
\hline MCl vs. AD & MacOSX10.6 & RadCluster \\
\hline MacOSX10.5 & $0.80-0.94$ & $0.75-0.92$ \\
MacOSX10.6 & - & $0.78-0.90$ \\
\hline
\end{tabular}

CON vs. AD

\begin{tabular}{ccc} 
MacOSX10.5 & $0.82-0.96$ & $0.75-0.96$ \\
MacOSX10.6 & - & $0.68-0.97$ \\
\hline
\end{tabular}

CON: controls; MCl: Mild cognitive impairment; AD: Alzheimer's disease. 
Table 3. Cortical thickness surface cluster similarities (Dice coefficients) across FreeSurfer versions

\begin{tabular}{lccc}
\hline $\mathrm{MCl}$ vs. AD & v4.5.0 & v5.0.0 & v5.1.0 \\
\hline v4.3.1* & $0.78-0.93$ & $0.69-0.80$ & $0.78-0.85$ \\
v4.5.0 & - & $0.66-0.82$ & $0.73-0.85$ \\
v5.0.0 & - & - & $0.78-0.89$ \\
\hline CON vs. AD & & & \\
\hline v4.3.1* & $0.75-0.89$ & $0.61-0.78$ & $0.70-0.86$ \\
v4.5.0 & - & $0.58-0.75$ & $0.63-0.82$ \\
v5.0.0 & - & - & $0.68-0.94$ \\
\hline
\end{tabular}

CON: controls; $\mathrm{MCl}$ : Mild cognitive impairment; AD: Alzheimer's disease.

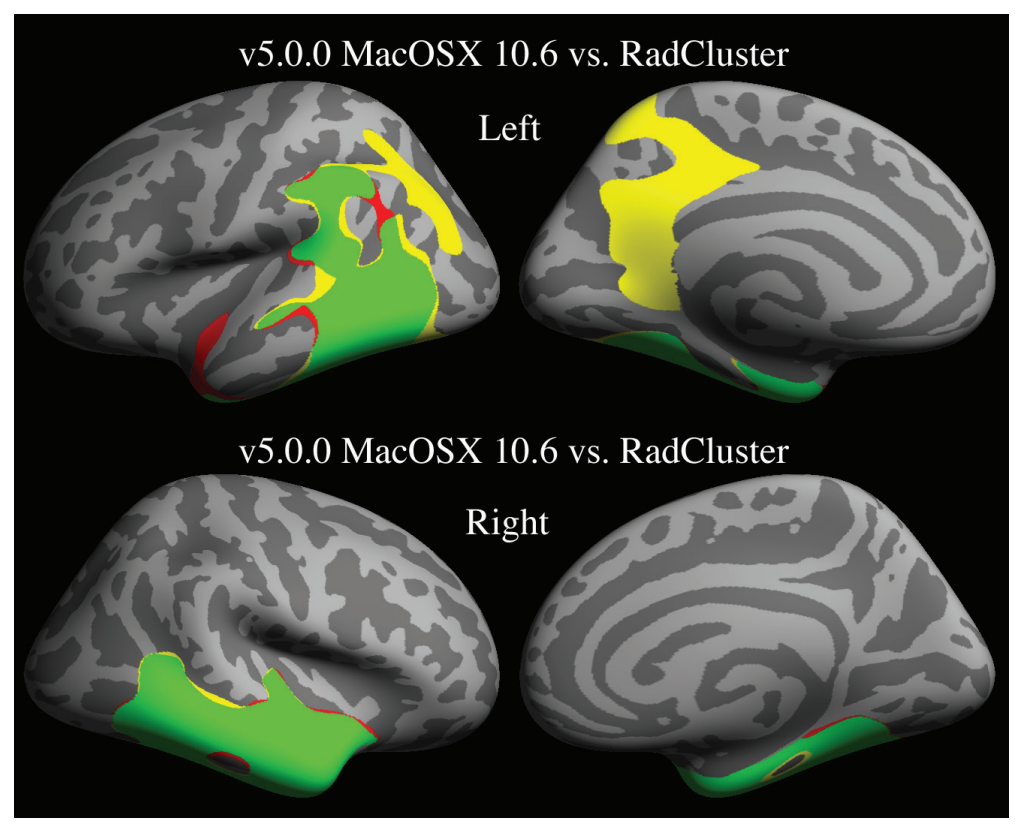

Fig. 3 CT Cluster similarities for the worst (top row) and best (bottom row) agreement found between workstations. The worst agreement was found for FreeSurfer v5.0.0 between MacOSX 10.6 (yellow) and RadCluster (red) and left hemisphere: $\mathrm{SI}=0.68$. The best agreement was found for FreeSurfer v5.0.0 between MacOSX 10.69 (yellow) and RadCluster (red) and right hemisphere: $\mathrm{SI}=0.97$. Vertices colored green indicate the presence of the clusters in the results of both workstations. 
Comparison of FreeSurfergroup differences with gold standard measurements When comparing AD-patients with controls, a good agreement was found between later versions of FreeSurfer and manual measurements, especially in MTL and posterior parietal regions. It is however remarkable that compared to the highly significant effect found for manual volumes, no group differences were found in the left OPFC for the automated measurements except in one instance, i.e., CT-vertex approach on MacOSX 10.5, version v4.5.0. For the right PCC, the opposite pattern was found where manual volumetric measurements did not show group differences in the right PCC compared to the automated measurements (all versions and workstations except for the CT-vertex approach). Compared to manual volumetric measurements, FreeSurfer seems less sensitive for detecting differences in prefrontal regions (both volume and CT). For the FreeSurfer volumetric and CT-parcellation measurements, group differences between CON vs. $\mathrm{MCl}$ were most apparent in posterior parietal regions ( $\mathrm{PCC}$ and $\mathrm{PC}$ ) whereas for the manual volumetric measurements, differences in the MTL were found (right PhG). The CT-vertex technique was unable to detect group differences between CON vs. $\mathrm{MCl}$. When $\mathrm{MCl}$ and $\mathrm{AD}$-patients were compared, group differences were mainly found in posterior parietal regions for the FreeSurfer volumetric measurements, compared to differences in MTL and prefrontal regions found with the manual volumetric measurement. For the comparison $\mathrm{MCl}$ vs. $\mathrm{AD}$, the $\mathrm{CT}$-vertex technique corresponded best with manual volumetric measurements and was even more sensitive for group differences than manual volumetry in posterior parietal regions. The CT-parcellation technique was unable to detect group differences between $\mathrm{MCl}$ vs. AD. 


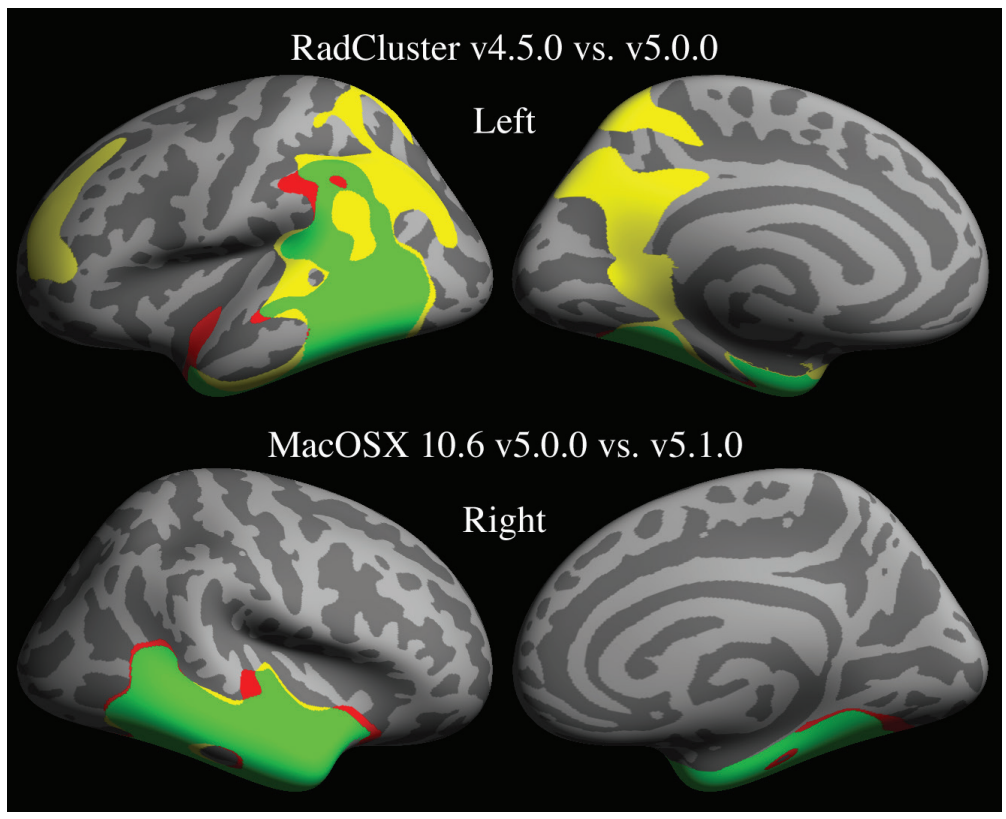

Fig. 4 CT Cluster similarities for the worst (top row) and best (bottom row) agreement found between FreeSurfer versions. The worst agreement was observed for RadCluster between versions v4.5.0 (yellow) and v5.0.0 (red) and left hemisphere: $\mathrm{SI}=0.58$. The best agreement was observed for MacOSX between versions v5.0.0 (yellow) and v5.1.0 (red) and right hemisphere: $\mathrm{SI}=0.94$. Vertices colored green indicate the presence of the clusters in the results of both workstations. The colors yellow and red denote the clusters found in only one of the workstations.

\section{DISCUSSION}

Alzheimer's disease pathology has a massive impact on both the structure and function of the ageing brain. Performance of automated brain segmentation techniques may be less precise when applied in these patients due to fundamental changes in certain brain structures. FreeSurfer has been shown to operate as an accurate instrument to measure brain atrophy in neurodegenerative diseases [35, 41-44], emphasizing the need for both validation and comparison of the FreeSurfer pipeline with manual volumetry in a cohort of subjects with and without Alzheimer's disease pathology. To the best of our knowledge, this is the first study evaluating the robustness of FreeSurfer to capture morphological and pathological changes against varying processing conditions, both in healthy old subjects and subjects suffering from (early) AD. For direct comparison, atrophy was also measured by 
means of manual volumetry, nowadays regarded as gold standard.

Overall, consistent results across versions and workstations were obtained for the hippocampus and PCC in case of volumetry. These structures seem less affected by changes in processing parameters, a finding of particular relevance for the imaging field of $A D$ since these structures are known to be primarily involved in the disease process $[4,45]$. With respect to $C T$ vertex-wise analyses, consistency was observed across versions and workstations for the PhG, left PCC, and left PC. In addition, the CTvertex technique was much more sensitive than the CT-parcellation technique. This finding is in line with previous work which found that CT-vertex was more sensitive than CT-parcellation in discriminating AD and controls [46]. The partial disagreement between CT-parcellation and manual volumetry may be related to the fact that effective validation of the $\mathrm{CT}$ technique requires postmortem investigations, and that manual volumetric measurements are thus not the most appropriate source of comparison. Furthermore, although differences between ROl's were small, borders of manual and FreeSurfer measurements were not entirely overlapping.

The FreeSurfer v5.1.0 results for MacOSX 10.6 are almost identical to those for RadCluster; only the CT-vertex results for the right PCC and PC deviate. In addition, a higher level of agreement was found between MacOSX 10.6 and Radcluster than between MacOSX 10.5 and 10.6. This supports the hypothesis in our previous paper, namely that most probably the number of bits under which the operating system is running (either 32 bits or 64 bits) is an important factor [7]. Although both MacOSX 10.5 and 10.6 were used in 32 bits mode, our results may suggest that the underlying UNIX shell for MacOSX 10.6 nonetheless uses mathematical libraries with 64 bits precision.

Only few studies reported on the capability of FreeSurfer to discriminate groups in comparison to manual volumetric measurements [10-12, 14, 47]. In general, these studies suggest that manual volumetry is slightly superior or equally sensitive to FreeSurfer automated volumetric measurements. While the previous studies focussed on one ROI (e.g. hippocampus), the present study extends these findings by examining all cortical signature regions in $A D$, and thereby increasing the diagnostic relevance and generalizability. 
For the comparison CON vs. AD, the present study found that manual volumetry and later versions of FreeSurfer (v5.0.0 and v5.1.0) were both sensitive in detecting hippocampal and parahippocampal volume reduction. Group differences in the posterior parietal lobe were less consistent between FreeSurfer and manual volumetry and it was found that later versions of FreeSurfer (v5.0.0 and v5.1.0) corresponded best with gold standard measurements. Furthermore, FreeSurfer was less sensitive for group differences in prefrontal regions compared to manual volumetric measurements (comparison $\mathrm{CON}$ vs. $A D$ and $\mathrm{MCl}$ vs. $A D$ ). In the early phase of the disease (CON vs. $\mathrm{MCl}$ ), FreeSurfer is more sensitive for group differences in posterior parietal regions compared to the MTL which is most pronounced in manual volumetry.

\section{Limitations}

One limitation may be related to the composition of the study population that comprised 18 healthy controls, 18 patients with amnestic $\mathrm{MCl}$, and 17 patients with mild AD. Only males were included in this study in order to reduce interindividual variation. Generalization of our findings to females should therefore be done with caution. Previous studies did however not reveal substantial gender related differences with respect to atrophy in AD [13]. The sample size of the present study is relatively small, which may possibly limit the ability to detect differences between groups that might become apparent when sample sizes are larger. Nonetheless, data was normally distributed and based on the inclusion of a time-intensive technique such as manual volumetry and on our hypothesis, sample size was fair enough to effectively address our research questions. Neurodegenerative processes in AD do not occur randomly, but likewise do not follow the boundaries set out by atlases. This is in line with the notion that locations of neurodegeneration may not coincide with predefined ROIs and thus such ROIs may harbor intrinsically less discriminative power, i.e., local effects are averaged out. On the other hand, the discriminative power of the manually defined ROIs was high in all cases, except for the IPFC and right PCC. Obviously, differences in the locations of the ROI boundaries between FreeSurfer and manual outlining may play a role, leading to overestimation of the volumes by the automated technique $[8,10,47,48]$. To correct for multiple comparisons, we performed the FDR controlling procedure instead of the classical Bonferonni approach because the former is less stringent and as such reduces the increased probability of making Type II errors $[49,50]$. 
As a consequence, correction for multiple comparisons was done over 14 comparisons for the FS automated volumetry and 12 for manual volumetry, which is slightly in favor of the manual technique. The two latest versions of FreeSurfer (5.2.0 and 5.3.0) were not included in the present analyses. However, a literature search (PUBMED) showed us that near half of the published FreeSurfer papers in the field of $A D$ thus far used one of the versions evaluated in this paper $(n=34) .31$ papers did not specify the version used, 3 used newer versions and 17 studies used earlier releases (e.g. version 3.0). This finding clearly emphasizes the importance of the results reported in the present study.

\section{Implications for research and clinical practice}

From the present study follows that despite the large morphological changes on a brain level, FreeSurfer can reliably be used to investigate structural brain alterations in AD. Another important finding is that, compared to manual volumetry, later versions of FreeSurfer are more accurate than earlier versions. This development is very promising for future applications of FreeSurfer in scientific studies on neurodegenerative diseases, and encourages the future role of FreeSurfer in clinical settings. While FreeSurfer is created for use in

research settings, the potential of FreeSurfer for use in clinical practice is promising. Based on our findings, we would suggest focusing on automated volumetry of posterior parietal regions in the early phase, followed by segmentation of MTL regions and measurements of $\mathrm{CT}$ in the later stages. The potential of CT-vertex measurements as a marker of disease progression should be further investigated in large-scale longitudinal studies. Prior work of our group has shown that mixing up versions, workstations or operating systems might lead to significant differences in cortical thickness or volumetry in schizophrenic patients [7]. However, the current findings are much more optimistic, showing that the latest version of FS produces much more stable results, even in patients with heavily atrophied brains. While this is an important scientific finding, we agree with the FreeSurfer developers that it is still advised to try to keep the research environment stable

\section{CONCLUSION}

The present study showed that FreeSurfer is a robust method to measure morphological and pathological changes in both healthy and atrophied brains. 
Overall, consistent results across versions and workstations were obtained particularly for the hippocampus and PCC in case of volumetry. Later versions of FreeSurfer (v5.0.0 and v5.1.0) corresponded best with gold standard measurements (manual volumetry), especially in MTL regions. In addition, the CT-vertex technique was much more sensitive than the CT-parcellation technique. The continuous efforts of the developers of FreeSurfer to improve its performance are very promising for $A D$ research and the neuroimaging field in general, and thus potentially also for clinical practice.

\section{APPENDIX}

The analysed cortical and subcortical ROls were composed of the following FreeSurfer segmentation IDs with their corresponding structure names:

HIPP: 17 (left hippocampus) / 53 (right hippocampus)

PHG: 1016 (left parahippocampal) / 2016 (right parahippocampal)

IPFC: 1018 (left pars opercularis) / 2018 (right pars opercularis) 1020 (left pars triangularis) / 2020 (right pars triangularis)

OPFC: 1012 (left lateral orbitofrontal) / 2012 (right lateral orbitofrontal) 1014 (left medial orbitofrontal) / 2014 (right medial orbitofrontal)

PCC: 1010 (left isthmus cingulate) / 2010 (right isthmus cingulate)

PC: 1025 (left precuneus) / 2025 (right precuneus) 


\section{REFERENCES}

1. Wimo A, Winblad B, Aguero-Torres H, von Strauss E. The magnitude of dementia occurrence in the world. Alzheimer disease and associated disorders 2003;17:63-67.

2. Jack CR, Knopman DS, Jagust WJ, et al. Hypothetical model of dynamic biomarkers of the Alzheimer's pathological cascade. The Lancet Neurology 2010;9:119-128.

3. Clerx L, Visser PJ, Verhey F, Aalten P. New MRI Markers for Alzheimer's Disease: A Meta-Analysis of Diffusion Tensor Imaging and a Comparison with Medial Temporal Lobe Measurements. Journal of Alzheimer's Disease 2012;29:405429.

4. Jacobs HI, Van Boxtel MP, van der Elst W, et al. Increasing the diagnostic accuracy of medial temporal lobe atrophy in Alzheimer's disease. J Alzheimers Dis 2011;25:477-490.

5. Boccardi M, Ganzola R, Bocchetta M, et al. Survey of Protocols for the Manual Segmentation of the Hippocampus: Preparatory Steps Towards a Joint EADCADNI Harmonized Protocol. Journal of Alzheimer's Disease 2011;26:61-75.

6. Barnes J, Ourselin S, Fox NC. Clinical application of measurement of hippocampal atrophy in degenerative dementias. Hippocampus 2009;19:510-516.

7. Gronenschild EHBM, Habets P, Jacobs HIL, et al. The Effects of FreeSurfer Version, Workstation Type, and Macintosh Operating System Version on Anatomical Volume and Cortical Thickness Measurements. PLoS ONE 2012;7:e38234.

8. Morey RA, Petty $\mathrm{CM}, \mathrm{Xu}$, et al. A comparison of automated segmentation and manual tracing for quantifying hippocampal and amygdala volumes. Neuroimage 2009;45:855-866.

9. Fischl B. FreeSurfer. Neuroimage 2012;doi:10.1016/j.neuroimage.2012.01.021.

10. Lehmann M, Douiri A, Kim LG, et al. Atrophy patterns in Alzheimer's disease and semantic dementia: A comparison of FreeSurfer and manual volumetric measurements. Neurolmage 2010;49:2264-2274.

11. Morey RA, Selgrade ES, Wagner HR, 2nd, Huettel SA, Wang L, McCarthy G. Scan-rescan reliability of subcortical brain volumes derived from automated segmentation. Hum Brain Mapp 2010;31:1751-1762.

12. Kim H, Chupin M, Colliot O, Bernhardt BC, Bernasconi N, Bernasconi A. Automatic hippocampal segmentation in temporal lobe epilepsy: impact of 
developmental abnormalities. Neuroimage 2012;59:3178-3186.

13. Albert M, Massaro J, DeCarli C, et al. Profiles by sex of brain MRI and cognitive function in the framingham offspring study. Alzheimer Dis Assoc Disord. 2010;24.

14. Pardoe HR, Pell GS, Abbott DF, Jackson GD. Hippocampal volume assessment in temporal lobe epilepsy: How good is automated segmentation? Epilepsia 2009;50:2586-2592.

15. Tisserand DJ, Pruessner JC, Sanz Arigita EJ, et al. Regional Frontal Cortical Volumes Decrease Differentially in Aging: An MRI Study to Compare Volumetric Approaches and Voxel-Based Morphometry. Neurolmage 2002;17:657-669.

16. Petersen RC. Alzheimer's disease: progress in prediction. Lancet Neurol 2010;9:4-5.

17. Jack J, C.R., Dickson DW, Parisi JE, et al. Antemortem MRI Findings Correlate with Hippocampal Neuropathology in Typical Aging and Dementia. Neurology 2002;58:750-757.

18. Echávarri C, Aalten P, Uylings HBM, et al. Atrophy in the parahippocampal gyrus as an early biomarker of Alzheimer's disease. Brain Structure and Function 2011;215:265-271.

19. Burgmans S, van Boxtel MPJ, Smeets F, et al. Prefrontal cortex atrophy predicts dementia over a six-year period. Neurobiology of Aging 2009;30:1413-1419.

20. Jacobs HIL, Van Boxtel MPJ, Jolles J, Verhey FRJ, Uylings HBM. Parietal cortex matters in Alzheimer's disease: An overview of structural, functional and metabolic findings. Neuroscience \& Biobehavioral Reviews 2011.

21. Jacobs HIL, Van Boxtel MPJ, van der Elst W, et al. Increasing the Diagnostic Accuracy of Medial Temporal Lobe Atrophy in Alzheimer's Disease. Journal of Alzheimer's Disease 2011;25:477-490.

22. Nylenna M, Riis P. Identification of patients in medical publications: need for informed consent. Bmj 1991;302:1182.

23. Fischl B, Salat DH, Busa E, et al. Whole brain segmentation: automated labeling of neuroanatomical structures in the human brain. Neuron 2002;33:341-355.

24. Desikan RS, Ségonne F, Fischl B, et al. An automated labeling system for subdividing the human cerebral cortex on MRI scans into gyral based regions of interest. Neurolmage 2006;31:968-980.

25. Dale AM, Fischl B, Sereno MI. Cortical surface-based analysis. I. Segmentation 
and surface reconstruction. Neuroimage 1999;9:179-194.

26. Fischl B, Dale AM. Measuring the thickness of the human cerebral cortex from magnetic resonance images. Proc Natl Acad Sci U S A 2000;97:11050-11055.

27. Fischl B, Sereno MI, Dale AM. Cortical surface-based analysis. II: Inflation, flattening, and a surface-based coordinate system. Neuroimage 1999;9:195207.

28. Han X, Jovicich J, Salat D, et al. Reliability of MRI-derived measurements of human cerebral cortical thickness: the effects of field strength, scanner upgrade and manufacturer. Neuroimage 2006;32:180-194.

29. Fischl B, Sereno MI, Tootell RB, Dale AM. High-resolution intersubject averaging and a coordinate system for the cortical surface. Hum Brain Mapp 1999;8:272284.

30. Gronenschild EH, Burgmans S, Smeets F, Vuurman EF, Uylings HB, Jolles J. A time-saving and facilitating approach for segmentation of anatomically defined cortical regions: MRI volumetry. Psychiatry Res 2010;181:211-218.

31. Burgmans S, van Boxtel MP, van den Berg KE, etal. The posterior parahippocampal gyrus is preferentially affected in age-related memory decline. Neurobiol Aging 2011;32:1572-1578.

32. Jones BF, Barnes J, Uylings $\mathrm{HB}$, et al. Differential regional atrophy of the cingulate gyrus in Alzheimer disease: a volumetric MRI study. Cereb Cortex 2006;16:1701-1708.

33. Ryu SY, Kwon MJ, Lee SB, et al. Measurement of precuneal and hippocampal volumes using magnetic resonance volumetry in Alzheimer's disease. J Clin Neurol 2010;6:196-203.

34. Shrout PE, Fleiss JL. Intraclass correlations: uses in assessing rater reliability. Psychological Bulletin 1979;2:420-428.

35. Clerx L, Jacobs HIL, Burgmans S, et al. Sensitivity of different MRI-techniques to assess gray matter atrophy patterns in Alzheimer's disease is region-specific. Current Alzheimer Research 2013;10:940-951.

36. Smith SM. Fast robust automated brain extraction. Human Brain Mapping 2002;17:143-155.

37. Benjamini Y, Hochberg Y. Controlling the False Discovery Rate: A Practical and Powerful Approach to Multiple Testing. Journal of the Royal Statistical Society. Series B (Methodological) 1995;57:289-300.

38. Hagler DJ, Jr., Saygin AP, Sereno MI. Smoothing and cluster thresholding for Chapter 7: FreeSurfer vs. manual volumetry | 241 
cortical surface-based group analysis of fMRI data. Neuroimage 2006;33:10931103.

39. Hammers A, Heckemann R, Koepp MJ, et al. Automatic detection and quantification of hippocampal atrophy on MRI in temporal lobe epilepsy: a proof-of-principle study. Neuroimage 2007;36:38-47.

40. Zijdenbos AP, Dawant BM, Margolin RA, Palmer AC. Morphometric analysis of white matter lesions in MR images: method and validation. IEEE Trans Med Imaging 1994;13:716-724.

41. Carmichael O, Xie J, Fletcher E, Singh B, DeCarli C. Localized hippocampus measures are associated with Alzheimer pathology and cognition independent of total hippocampal volume. Neurobiology of Aging 2012;33:1124-1141.

42. Murray AD, Staff RT, McNeil CJ, et al. The balance between cognitive reserve and brain imaging biomarkers of cerebrovascular and Alzheimer's diseases. Brain 2011;134:3687-3696.

43. Westman E, Muehlboeck JS, Simmons A. Combining MRI and CSF measures for classification of Alzheimer's disease and prediction of mild cognitive impairment conversion. Neurolmage 2012;62:229-238.

44. Zahodne LB, Gongvatana A, Cohen RA, Ott BR, Tremont G. Are Apathy and Depression Independently Associated With Longitudinal Trajectories of Cortical Atrophy in Mild Cognitive Impairment? The American Journal of Geriatric Psychiatry 2013.

45. Clerx L, van Rossum I, Burns L, et al. Measurements of medial temporal lobe atrophy in the prediction of Alzheimer's Disease in subjects with $\mathrm{MCl}$. Neurobiology of Aging 2013.

46. Cuingnet R, Gerardin E, Tessieras J, et al. Automatic classification of patients with Alzheimer's disease from structural MRI: a comparison of ten methods using the ADNI database. Neuroimage 2011;56:766-781.

47. Shen L, Saykin A, Kim S, et al. Comparison of Manual and Automated Determination of Hippocampal Volumes in $\mathrm{MCl}$ and Early $\mathrm{AD}$. Brain Imaging and Behavior 2010;4:86-95.

48. Sanchez-Benavides G, Gomez-Ansun B, Sainz A, Vives Y, Delfino M, PeoaCasanova J. Manual validation of FreeSurfer's automated hippocampal segmentation in normal aging, mild cognitive impairment, and Alzheimer Disease subjects. Psychiatry Research: Neuroimaging 2010;181:219-225.

49. Garamszegi LZ. Comparing effect sizes across variables: generalization without 
the need for Bonferroni correction. Behavioral Ecology 2006;17:682-687.

50. Nakagawa S. A farewell to Bonferroni: the problems of low statistical power and publication bias. Behavioral Ecology 2004;15:1044-1045. 


\section{SUPPLEMENTAL MATERIAL}

For Supplemental Table 1. 'Anatomical boundaries of investigated structures' please see Chapter 4, page 126-130.

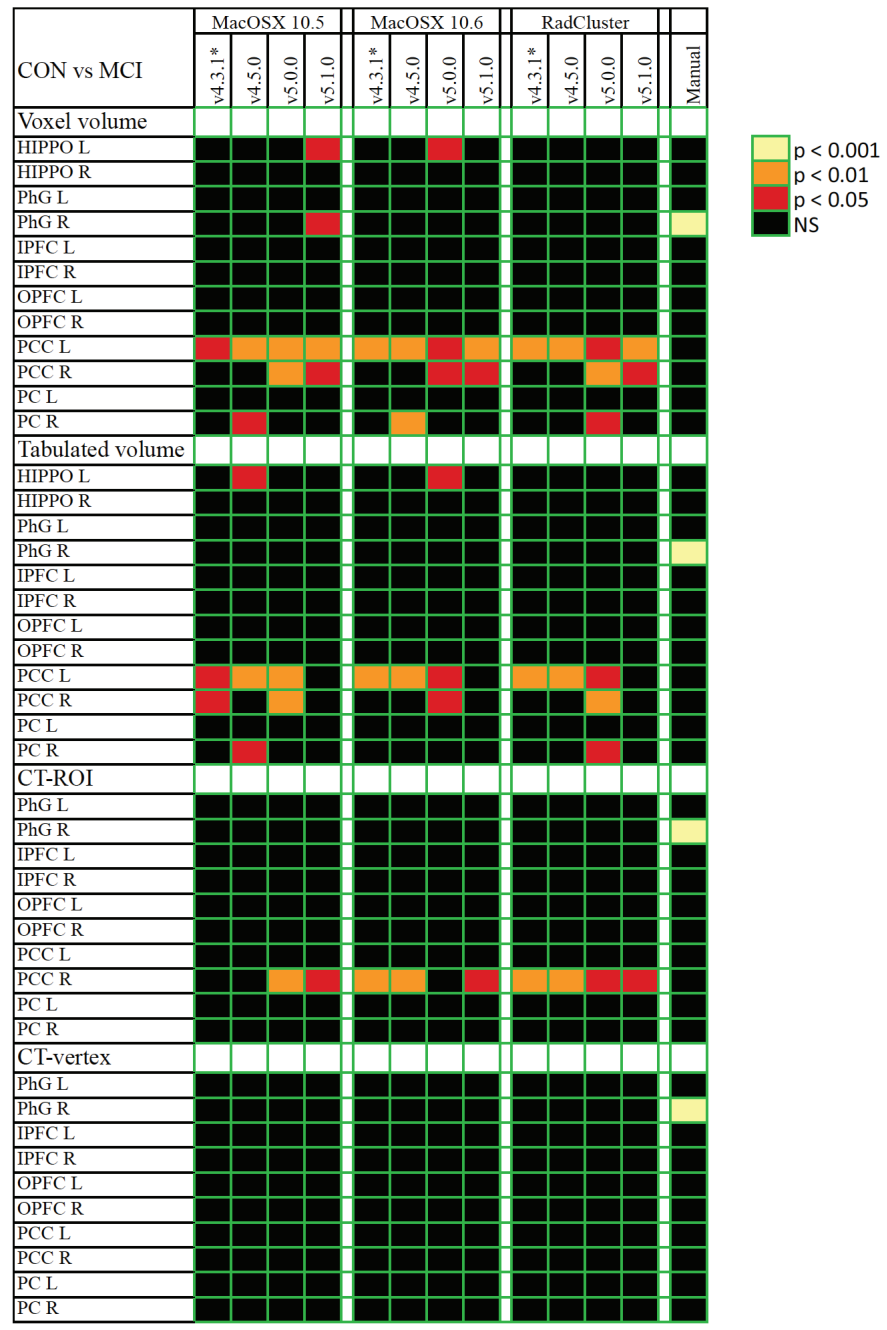

Supplementary Figure. 1 Overview of statistical significances across FreeSurfer versions and workstations for the comparison of controls versus $\mathrm{MCl}$. Each cell is color-coded according to its $p$-value in four categories, see coding scale at right. The first set of four columns refers to the MacOSX 10.5 workstation, the second set to the MacOSX 10.6 workstation, and the third set to the custom-built cluster workstation (called RadCluster). In each set, the columns present the results for version v4.3.1*, v4.5.0, v5.0.0, and v5.1.0, respectively. Finally, the last column refers to the $p$-values based on the manual outlining. Only the results after 
correction for multiple comparisons are shown. Four separate FreeSurfer measurements were used: 1) number of voxels within a segmented ROI (called voxel volume); 2 ) tabulated $\mathrm{ROI}$ volume (equal to voxel volume but corrected for partial volume effects); 3 ) mean cortical thickness (CT) within an ROI (called CT-parcellation-based); 4) vertex-wise CT cluster (called CT-vertex). Abbreviations are as follows: $\mathrm{HIPPO}=$ hippocampus; $\mathrm{ERC}=$ entorhinal cortex; PhG = parahippocampal gyrus; IPFC = inferior prefrontal cortex; OPFC = orbital prefrontal cortex; $P C C=$ posterior cingulate cortex; $P C=$ precuneus; $L=$ left; $R=$ right.

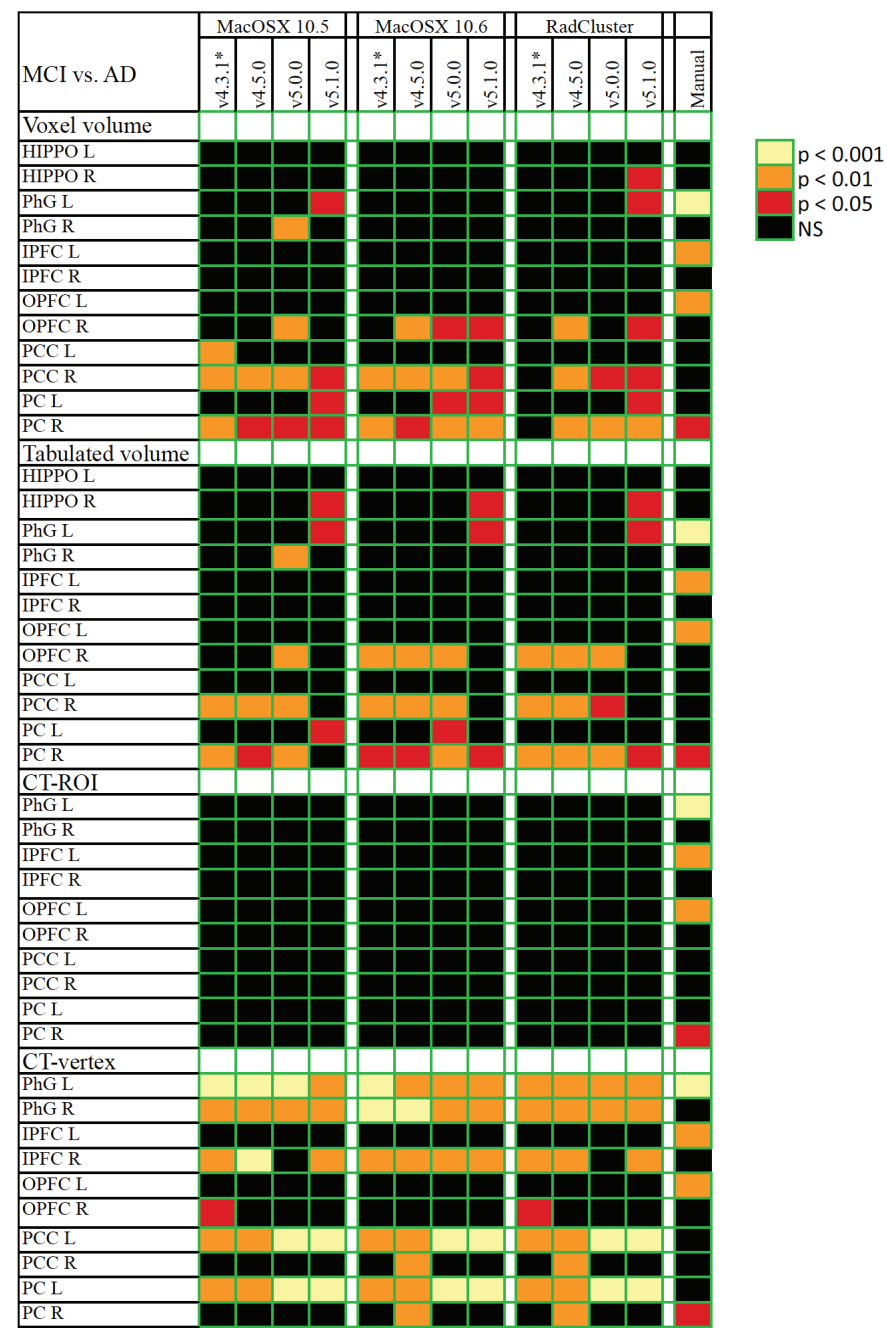

Supplementary Figure. 2 Overview of statistical significances across FreeSurfer versions and workstations for the comparison of $\mathrm{MCl}$ versus $\mathrm{AD}$. Each cell is color-coded according to its $\mathrm{p}$-value in four categories, see coding scale at right. The first set of four columns refers to the MacOSX 10.5 workstation, the second set to the MacOSX 10.6 workstation, and the third set to the custom-built cluster workstation (called RadCluster). In each set, the columns 
present the results for version v4.3.1*, v4.5.0, v5.0.0, and v5.1.0, respectively. Finally, the last column refers to the $p$-values based on the manual outlining. Only the results after correction for multiple comparisons are shown. Four separate FreeSurfer measurements were used: 1) number of voxels within a segmented ROI (called voxel volume); 2 ) tabulated ROI volume (equal to voxel volume but corrected for partial volume effects); 3 ) mean cortical thickness (CT) within an ROI (called CT-parcellation-based); 4) vertex-wise CT cluster (called CT-vertex). Abbreviations are as follows: HIPPO = hippocampus; $\mathrm{ERC}=$ entorhinal cortex; PhG = parahippocampal gyrus; IPFC = inferior prefrontal cortex; OPFC = orbital prefrontal cortex; $P C C=$ posterior cingulate cortex; $P C=$ precuneus; $L=$ left; $R=$ right. 



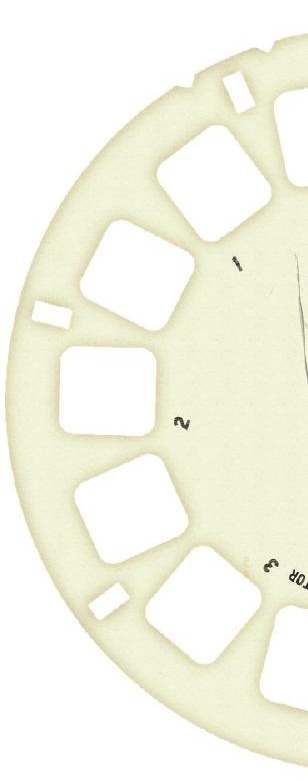




\begin{abstract}
Reduced functional connectivity (FC) and atrophy in hubs of the default mode network (DMN) is a consistent and early finding in Alzheimer's disease (AD). In order to use FC changes in the DMN as candidate biomarker for $A D$, its stability in normal aging needs to be assessed. Test-retest-reliability of RSNs was investigated both in healthy young $(\mathrm{HY})$ and healthy old $(\mathrm{HO})$ subjects. Twenty HY and twenty HO subjects underwent three scanning sessions on a 3.0 Tesla MRI. Differences in FC were investigated in eight RSNs. Within-group analyses showed no change over time (intraclass correlation coefficients: 0.53-0.85). Between-group comparisons showed more $\mathrm{FC}$ in the DMN and auditory system in $\mathrm{HY}$ compared to $\mathrm{HO}$ subjects. Inverse correlations were found between instability of specific DMN areas and neuropsychology:more variability in precuneal connectivity was linked with lower performance. As expected, FC in the DMN and auditory system decreases with aging. Activity in major RSNs remains stable over time, and instability in key hubs of the DMN could become a predictor for future memory problems, supporting its potential as a future biomarker.
\end{abstract}




\section{INTRODUCTION}

Brain activation in the past has mainly been studied using the BOLD signal in taskrelated fMRI paradigms, aimed at localizing a variety of memory functions including age associated effects [1-3]. The last decade, task-related decreases are found in certain regions which remain active in an organized fashion during the resting state of the brain [1, 2]. One of these so-called 'resting state networks' (RSNs) is the default mode network (DMN), particularly relevant for aging and dementia since this network is vulnerable to atrophy, deposition of the amyloid protein, and show a reduced glucose metabolism [3]. Furthermore, the DMN comprises several areas that are also affected early on in Alzheimer's disease (AD), such as the posterior cingulate cortex, medial and lateral prefrontal cortex, medial inferior parietal lobe, precuneus, and the medial temporal lobe (Hafkemeijer, et al., 2012; Jacobs, et al., 2013). Because of this reason, the DMN is considered to hold great potential as clinical biomarker. Besides the DMN, the brain consists of a number of other low frequency RSNs, such as the visual and auditory networks [4]. The potential effects of normal aging on these functional hubs have not thoroughly been investigated [5].

The first step toward a biomarker is assuring that age-effects do not influence the reliability of RSNs. In view of a number of studies demonstrating the utility of RSNs $[6,7]$, it remains to be investigated if rs-fMRI is a stable and reliable technique for identifying and characterizing abnormalities in a variety of diseases. As it is widely known that pathophysiological processes of neurodegenerative diseases (e.g. AD) begin many years prior to clinical symptoms, efforts currently aim at detecting AD-pathology in clinically (still) asymptomatic individuals. The ultimate goal of establishing these so-called 'biomarkers' is the identification of and intervention in individuals at the preclinical stage to delay and possibly even prevent onset of the clinical syndrome.

The present study aimed at investigating the test-retest reliability (i.e. stability) of RSNs within and between healthy old and healthy young subjects over 3 different time points. Test-retest reliability was investigated both on a voxel-wise basis (FSL) and by means of the intraclass correlation coefficient (ICC) (SPSS). As the DMN is the most investigated RSN and thus far has the closest link to AD, our second aim was to investigate whether individual changes in stability in certain hubs of the DMN are 
associated with cognitive performance. If instability over time is linked with subtle cognitive alterations in healthy old subjects, this finding would be of particular relevance for early diagnosis and intervention in at-risk individuals.

\section{METHODS AND MATERIALS}

\section{Subjects}

Forty healthy subjects were included and gender-matched: 20 elderly subjects (HO) (age between 54-66 years) and 20 young subjects (HY) (age between 23-29 years). Subjects were recruited for the RIMCAD study (Retroactive Interference during Memory Consolidation in Aging and Dementia) by means of advertisement. Exclusion criteria were significant medical (e.g. cancer, thyrotoxicosis), neurological (e.g. epilepsy), or other psychiatric disorders (e.g. schizophrenia, depression, Parkinson's disease); current use of medication which is known to influence cerebral function (e.g. anti-depressants); pregnancy or any other contraindication against MRI-scanning (e.g. metal implants, claustrophobia). One subject in the elderly group was excluded because MRI images were not available for all three time points. The Ethical Review Board of the University Hospital of Cologne had approved the study, and the study was accomplished in compliance with the latest revision of the Declaration of Helsinki. Written informed consent was provided by all subjects.

\section{Neuropsychological assessment and study design}

All subjects received neuropsychological examination to confirm their healthy status, including Mini-mental state examination (MMSE) [8], immediate and delayed recall of the German version of the Verbal Learning and Memory test [9], Trail Making Test (TMT) part A and B [10] and Rey-Osterrieth Complex Figure test [11]. Three separate experimental sessions were organized, with on average two weeks in between each session. At the time of the first visit subjects were tested neuropsychologically whereafter the first MRI examination was performed. At the second and third visit ( 2 weeks and 4 weeks from baseline, respectively) only MRI scanning was performed.

\section{MRI data acquisition}

All imaging was performed using a 3.0 Tesla Siemens Trio MRI scanner (Siemens, Erlangen, Germany) equipped with a standard head coil for radio frequency

252 | Chapter 8: Stability of resting state networks in aging 
transmission and signal reception. For each participant, 3 resting-state scans were acquired (one per visit) (191 continuous T2*-weighted echo planar imaging (EPI) volumes; repetition time $=2200 \mathrm{~ms}$; echo time $=30 \mathrm{~ms}$; flip angle $=90^{\circ} ; 36$ axial slices; matrix 64 ×64; voxel size $3.1 \times 3.1 \times 3.0 \mathrm{~mm}$; scan time = 7 minutes). During the scans, participants were instructed to keep their eyes closed, think of nothing in particular, and not to fall asleep. In addition, a high-resolution T1 anatomical image was obtained for each subject using a three-dimensional magnetization-prepared, rapid acquisition gradient echo sequence. To control for cerebral abnormalities (e.g. tumor, subclinical stroke) and macroangiopathy, T2-weighted FLAIR and Timeof-Flight measurements were acquired in the older subjects only.

\section{Data preprocessing and analyses}

Data analysis was carried out using FMRIB's Software Library (FSL version 4.1.9; www. fmrib.ox.ac.uk/fsl). Preprocessing was carried out with MELODIC and consisted of motion correction, removal of nonbrain tissue (using the Brain Extraction Tool), spatial smoothing using a $5 \mathrm{~mm}$ full-width-at-half-maximum Gaussian kernel, and high-pass temporal filtering equivalent to 100 seconds $(0.01 \mathrm{~Hz})$. After preprocessing, $\mathrm{fMRI}$ volumes were registered to the subject's high-resolution T1-weighted scan using affine registration (FLIRT) and subsequently to standard space (MNI152) images using nonlinear registration (FNIRT) with a warp resolution of $10 \mathrm{~mm}$.

\section{Assessment of functional connectivity}

Functional connectivity of RSNs was defined in terms of the similarity of the BOLD fluctuations in each brain voxel in relation to characteristic fluctuations in eight predefined components (RSNs of interest). For the selection of these components an existing template was used containing a weighted mask of networks that are most reliably (and reproducibly) identified from a model-free analysis of the spatiotemporal structure of resting-state BOLD fluctuations [12]. These predefined components include over $80 \%$ of the total brain volume and comprise: medial and lateral visual systems (component 1 and 2, respectively), auditory and somatosensory system (component 3 ), sensori-motor system (component 4), default mode network (component 5), executive control (component 6), and right and left visuo-spatial and working memory networks (component 7 and 8, respectively). Components accounting for cerebrospinal fluid (CSF) and total brain white matter (WM) were furthermore included to capture activations for both sources of noise. 
To investigate $\mathrm{FC}$, a voxel-wise comparison of the resting state signal was carried out using a regression technique referred to as the "dual-regression" method [13, 14]. This technique is based on first extracting the temporal pattern of resting state signal fluctuations within a RSN (represented by each component in the predefined template) or CSF or WM, for each individual rs-fMRI dataset separately (spatial regression). Matrices describing the temporal dynamics for each component and subject separately were created and subsequently used in a linear model fit against the subject's fMRI data set (temporal regression) in order to estimate subjectspecific correlation maps. After this dual regression, spatial maps of all subjects were concatenated into single 4-dimensional files for each RSN. The regions that showed differences in FC between groups were visualized by means of FSLview and subsequently used to extract mean Z-values from each individual spatial map (FWE-corrected $p<0.05$ ). These values represent connectivity to the given RSN with higher absolute z-values reflecting stronger connectivity to a RSN.

\section{Statistical analysis}

To investigate our first aim, non-parametric permutation tests (5000 permutations) were used in order to detect statistically significant differences between the groups within the boundaries of the spatial maps defined by the predefined template (See Figure 6 in Beckmann et al., 2005 [12]). A family-wise error (FWE) correction for multiple comparisons was performed, implementing thresholdfree cluster enhancement (TFCE) using a significance threshold of $p<0.05$ [15]. First, main effects of group (old versus young), time (first, second and last time point) and the interaction group by time were examined. (Normalized) gray matter volume was added as an additional covariate in all permutation tests in order to correct for the effect of gray matter differences between subjects [16] (together with demeaned 'age' and/or 'education'; depending on the research question). Our second aim was to detect subtle differences in $\mathrm{FC}$ of the DMN within $\mathrm{HO}$ and $\mathrm{HY}$ subjects over time, and to correlate these individual changes in $\mathrm{FC}$ over time with neuropsychological test performance. For these additional statistical analyses on a subject-level, masks of selected regions-of-interest (ROI) were created by means of FSLview. Regions in the medial temporal lobe (hippocampus), posterior parietal lobe (precuneus and posterior cingulate gyrus) and frontal lobe (anterior cingulate gyrus) were selected based on their relevance for $A D[17,18]$. ROIs were placed on the T1_MNI152_2mm template by using the Harvard-Oxford (sub)cortical 
structural atlases, provided within FSL and resulting masks were subsequently used for extracting mean Z-scores for further analysis.

\section{(Non-imaging) statistical analysis}

To quantify test-retest reliability (or measurement reproducibility), intraclass correlation coefficients (ICC) were calculated for each individual resting state network $[19,20]$. First, the template containing each of the eight resting state networks was split into separate masks, one for each network, by means of fslsplit. Mean Z-scores of functional connectivity in the resulting masks (i.e. separate resting state networks) were extracted for each time point and were subsequently exported to SPSS (version 19.0; SPSS, Chicago, IL, USA). The ICC was calculated over the three different time points (SPSS - ICC, two-way mixed effects model for consistency). Different guidelines exist for the interpretation of ICC, but one reasonable scale is that an ICC value of less than 0.40 indicates poor reproducibility, ICC values in the range 0.40 to 0.75 indicate fair to good reproducibility, and an ICC value of greater than 0.75 shows excellent reproducibility [21].

ROI-based statistical analyses were performed using SPSS whereas correlation analyses were performed within both groups to assess relationships between regional FC within the DMN and neuropsychological test performance. Mean Z-scores of FC between a certain ROI and the rest of the DMN were extracted and transferred to SPSS. The absolute difference in FC between the first and the last time point was calculated (since we expected to find most variability between both utmost points of time) for each separate region with a higher value indicating larger differences between both time points, and thus more instability of FC over time for a given subject in a particular structure. To control for Type I errors, a false discovery rate (FDR) controlling procedure was performed in order to correct for multiple comparisons [22].

\section{RESULTS}

\section{Demographics and cognitive performance}

Subject characteristics are shown in Table 1. As expected from our study design, mean age of the old group differed significantly from that of the young group ( $p$ $<.001$ ). In the old group, education was lower compared to the younger group ( $p$ $<.01)$. Differences in cognitive performance between old and young subjects were 
found for the delayed recognition trial of the VLMT memory test $(p=.008)$ and the delayed recall of the Rey-Osterrieth Complex Figure test $(p=.04)$.

Identification of FC differences between $\mathrm{HO}$ and $\mathrm{HY}$ subjects across all time points

After correcting for $\mathrm{GM}$ and education, increased $\mathrm{FC}$ in older compared to younger subjects was found in all networks except the default mode network and the left dorsal visual stream (Table 2, Figure 1). Regions of increased FC in older subjects were mainly situated in frontal and occipital regions (Table 2, Figure 1). After correcting for $\mathrm{GM}$ and education, increased $\mathrm{FC}$ in younger compared to older people was found in all components except the lateral visual cortex (Table 2, Figure 2). These regions of increased FC in younger subjects were mainly situated in frontal and parietal regions (Table 2, Figure 2). For the whole group, no significant differences over time were found in each of the eight investigated networks. Likewise, no significant differences were found for the time $\mathrm{x}$ group interaction.

Table 1. Subject characteristics.

\begin{tabular}{lcc}
\hline & Old $(\mathrm{n}=19)$ & Young $(\mathrm{n}=20)$ \\
\hline Age (in years) & $59.2(6.1)^{* * *}$ & $24.6(2.8)$ \\
Education (in years) & $12.4(3.3)^{* *}$ & $15.6(2.7)$ \\
Female (in \%) & 47 & 50 \\
MMSE score & $29.4(0.7)$ & $29.5(0.9)$ \\
VLMT learning & $50.9(9.3)$ & $56.4(8.8)$ \\
VLMT memory & & \\
*delayed recall & $10.7(2.8)$ & $11.6(2.9)$ \\
(number of words) & & $13.8(1.3)$ \\
*delayed recognition & $11.7(3.1)^{* *}$ & $20.6(9.6)$ \\
(number of words) & $26.0(13.0)$ & $39.2(17.9)$ \\
TMT-A (sec.) & $53.4(29.0)$ & $35.5(0.9)$ \\
TMT-B (sec.) & $35.9(0.3)$ & $27.0(4.5)$ \\
CF Rey score IR & $22.5(6.3)^{*}$ & \\
CF Rey score DR &
\end{tabular}

Values are mean (sd). MMSE: Mini-Mental state examination; VLMT: verbal learning and memory test; TMT: trail making test; CF Rey: Rey complex figure test. Differences between both age groups significant at ${ }^{* * *} p<0.001,{ }^{* *} p<0.01$ and $* p<0.05$. 
Quantification of test-retest reliability by means of ICC

Test-retest reliability, expressed by means of the intraclass correlation coefficient (ICC), was investigated for both groups separately, and for each individual RSN. In HY subjects, ICCs varied from 0.53 (sensory-motor system) to 0.81 (medial visual system and auditory system). All networks, except the lateral visual system (0.66) and sensory motor system (0.53), showed excellent reproducibility in HY subjects (Table 3). For HO subjects, ICCs varied between 0.54 (executive control) and 0.85 (right dorsal visual stream). The medial visual cortex (0.82), auditory system (0.78) and dorsal visual stream (left/right) $(0.77 / 0.85)$ showed excellent reproducibility whereas the lateral visual cortex (0.73) and the default mode network (0.73) showed good reproducibility. The sensory-motor system (0.57) and executive control networks (0.54) showed fair reproducibility over time (Table 3). A visualization of test-retest reliability in the DMN is shown in Figure 3. Since the individual variability of FC within the DMN was most evident in $\mathrm{HO}$ subjects (subject number 1-19), the effect on neuropsychological test performance was further investigated. 


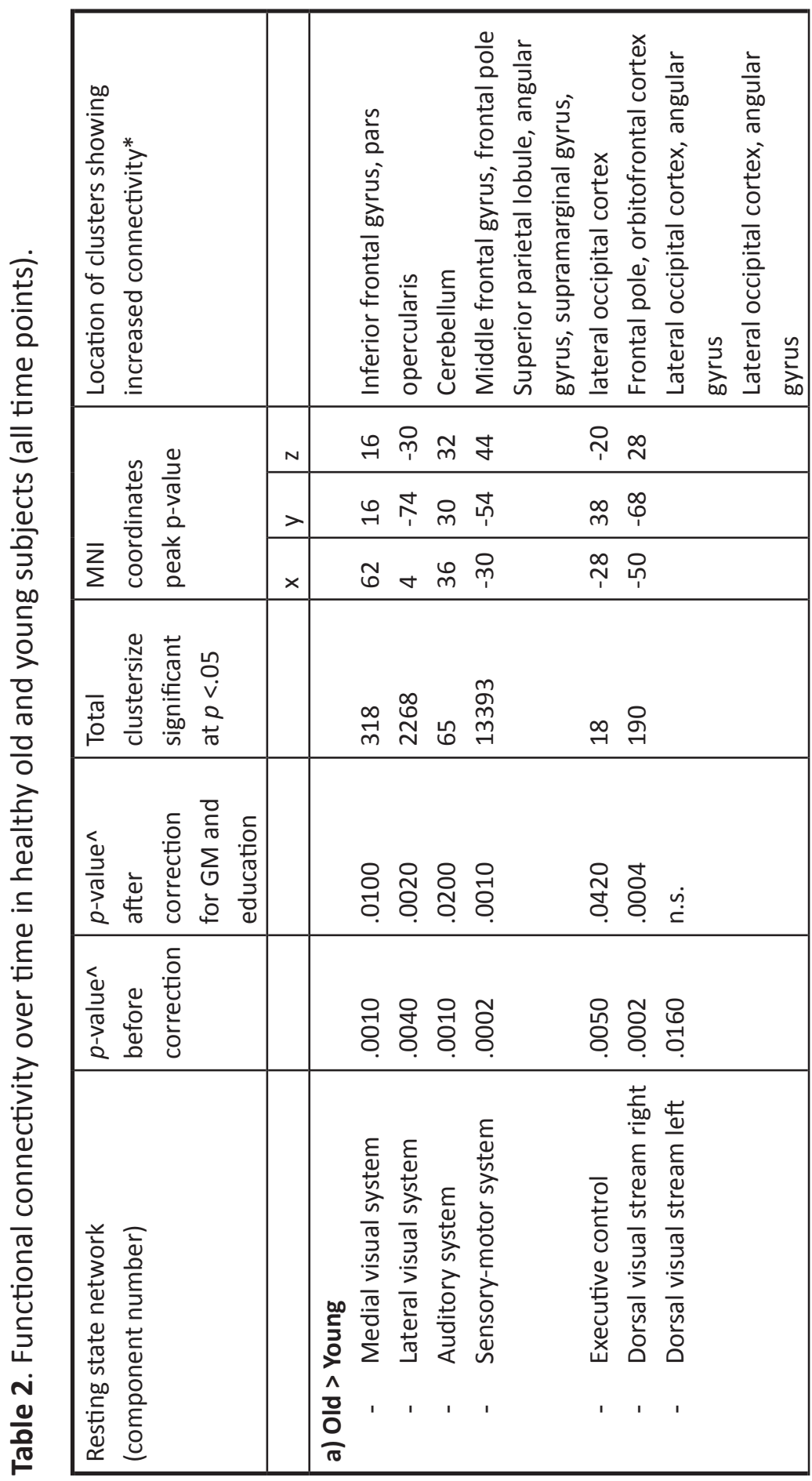

258 | Chapter 8: Stability of resting state networks in aging 


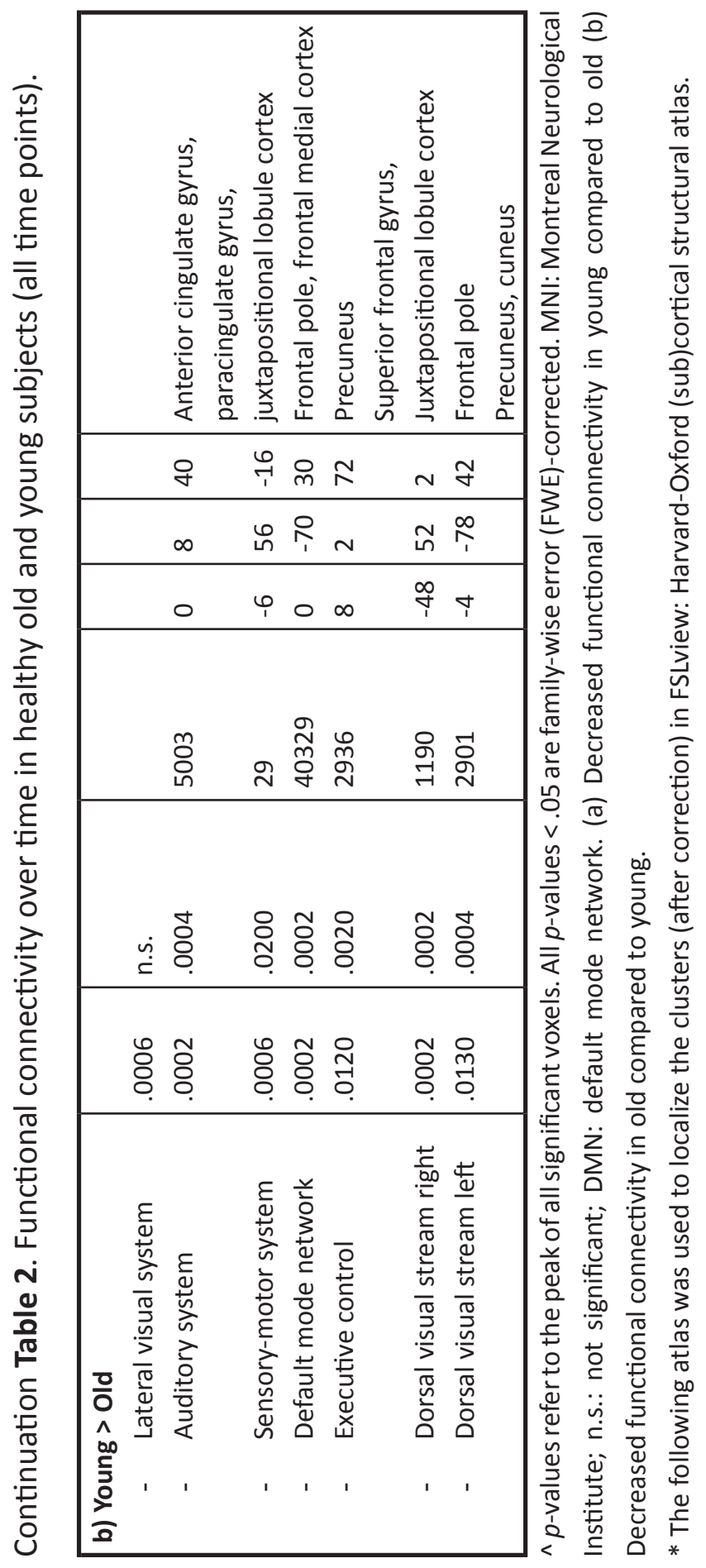

Chapter 8: Stability of resting state networks in aging | 259 
Table 3. Intraclass correlation coefficient (ICC) analysis for healthy old vs. healthy young subjects.

\begin{tabular}{lcc}
\hline & Old $(\mathrm{n}=19)$ & Young $(\mathrm{n}=20)$ \\
\hline Medial visual system & 0.82 & 0.81 \\
Lateral visual system & 0.73 & 0.66 \\
Auditory system & 0.78 & 0.81 \\
Sensory-motor system & 0.57 & 0.53 \\
Default mode network & 0.73 & 0.75 \\
Executive control & 0.54 & 0.8 \\
Dorsal visual stream right & 0.85 & 0.76 \\
Dorsal visual stream left & 0.77 & 0.77 \\
\hline
\end{tabular}

Values are depicted for each separate resting state network.
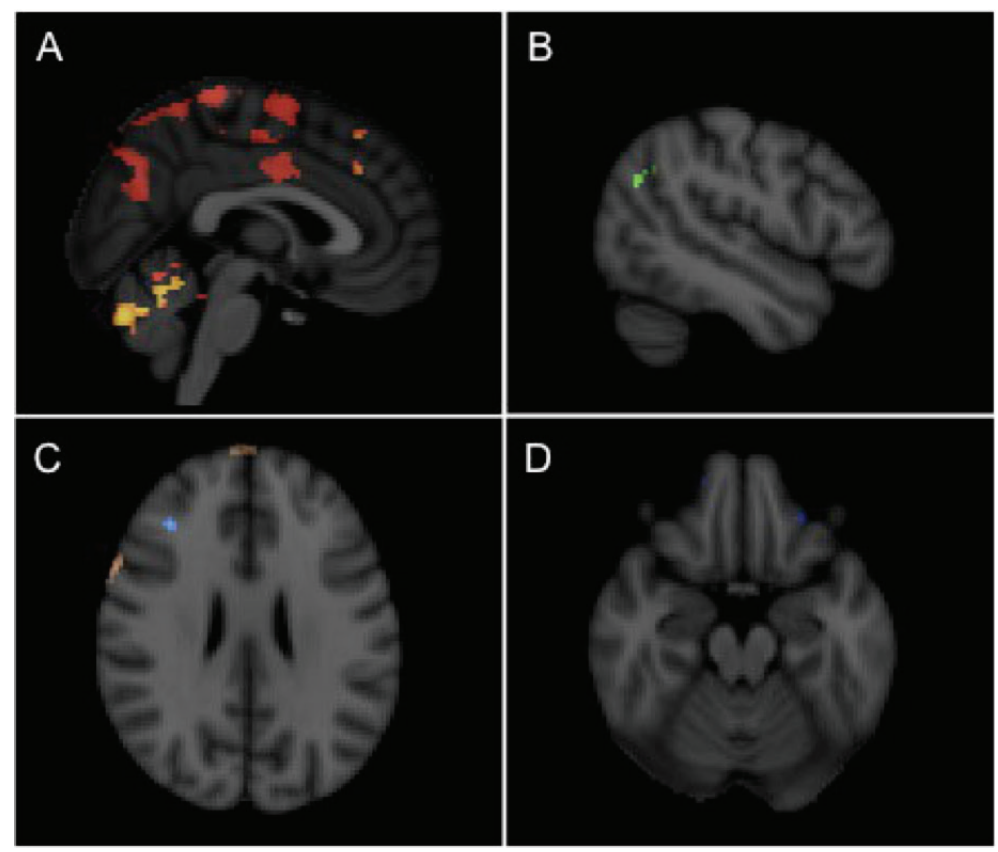

Figure 1. Regions showing increased functional connectivity in older subjects $(\mathrm{p}<.05$ familywise error [FWE]-corrected). (A) lateral visual cortical areas in yellow, sensory-motor system in red (B) right dorsal visual stream in green (C) medial visual system in brown, auditory system in blue, (D) executive control in dark blue. All images have been co-registered into the space of the MNI 152 template. The left hemisphere of the brain corresponds to the right side of the image. 


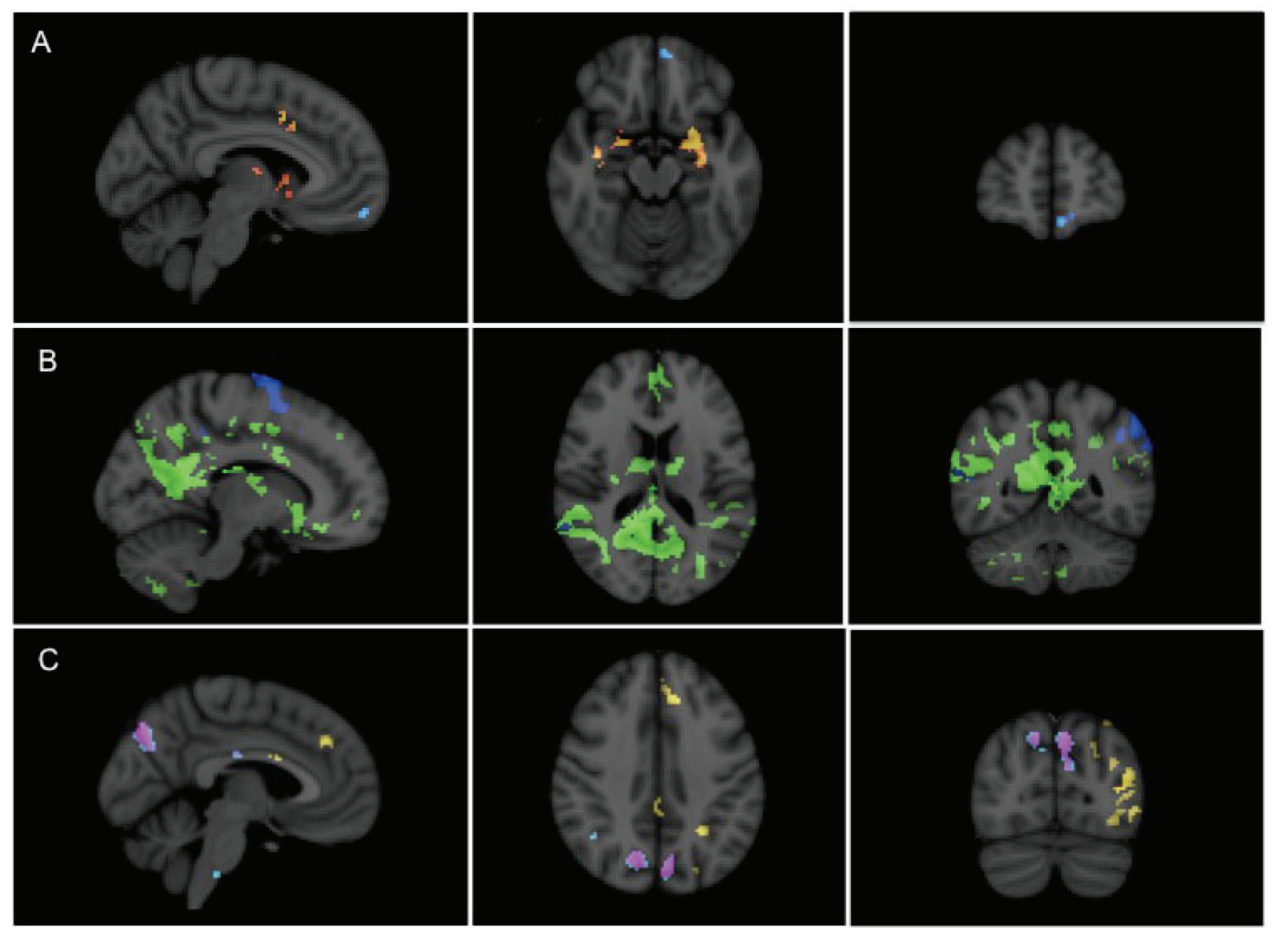

Figure 2. Regions showing increased functional connectivity in younger subjects $(p<.05$ family-wise error [FWE]-corrected). (A) auditory system depicted in red/yellow, sensorymotor system in blue (B) default mode network depicted in green, executive control in blue (C) right dorsal visual stream depicted in purple, left dorsal visual stream in yellow. All images have been coregistered into the space of the MNI 152 template. The left hemisphere of the brain corresponds to the right side of the image. The image shows saggital, coronal and axial views. 


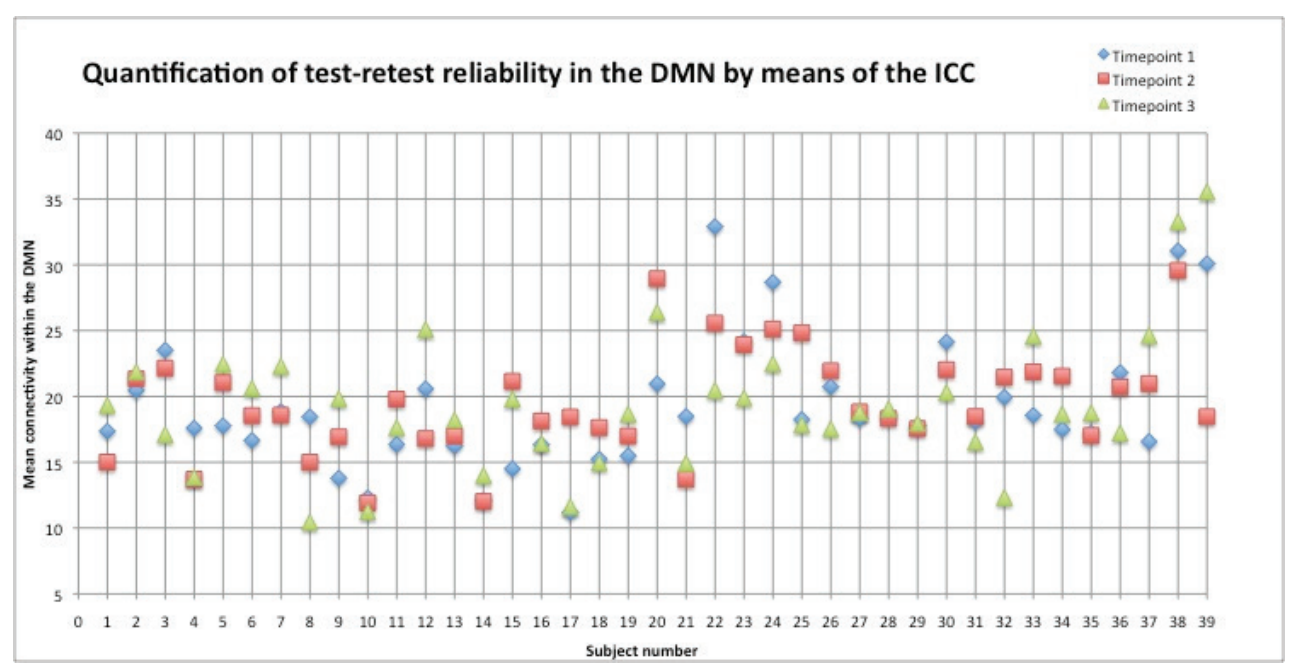

Figure 3. Quantification of test-retest reliability within the default mode network (DMN) by means of the intraclass correlation coefficient (ICC). Functional connectivity values within the DMN are depicted for each subject and time point separately. Subjects 1-19 belong to the group of healthy old subjects, subjects 20-39 belong to the group of healthy young subjects. The ICC reflecting test-retest reliability over all time points was 0.75 for healthy young subjects compared to 0.73 for healthy old subjects.

\section{Associations between stability of RSNs and NP test performance}

On a group level, no differences in stability were found over time. Next, in line with the findings above, we investigated on a subject-level whether stability differences in key hubs of the default mode network were linked with performance on neuropsychological tests.

Lower FC in older compared to younger subjects was mainly found in the DMN. ROls in the DMN were selected based on their relevance for AD. Correlation analyses in older subjects were performed in order to investigate if changes in FC between a ROI and the DMN over time were linked with neuropsychological test performance at baseline. Variability over time in $\mathrm{HO}$ subjects was assessed for each separate ROI and was expressed as absolute difference between time point 1 and 3 (Figure 4). It was found that higher variability over time in the precuneus was associated with a decrease in MMSE score $(r=-0.56, p<0.01)$, lower immediate $(r=-0.56, p<0.01)$ and delayed recall $(r=-0.55, p<0.05)$, and recognition scores 
$(r=-0.49, p<0.05)$ (Figure 5). After correcting for multiple comparisons, associations between precuneal connectivity and MMSE score, and between precuneal connectivity and immediate recall score remained significant $(p<0.05)$ (Figure 5$)$. No associations were found between variability in the hippocampus or cingulate cortex (ACC/ PCC) and neuropsychological test performance.

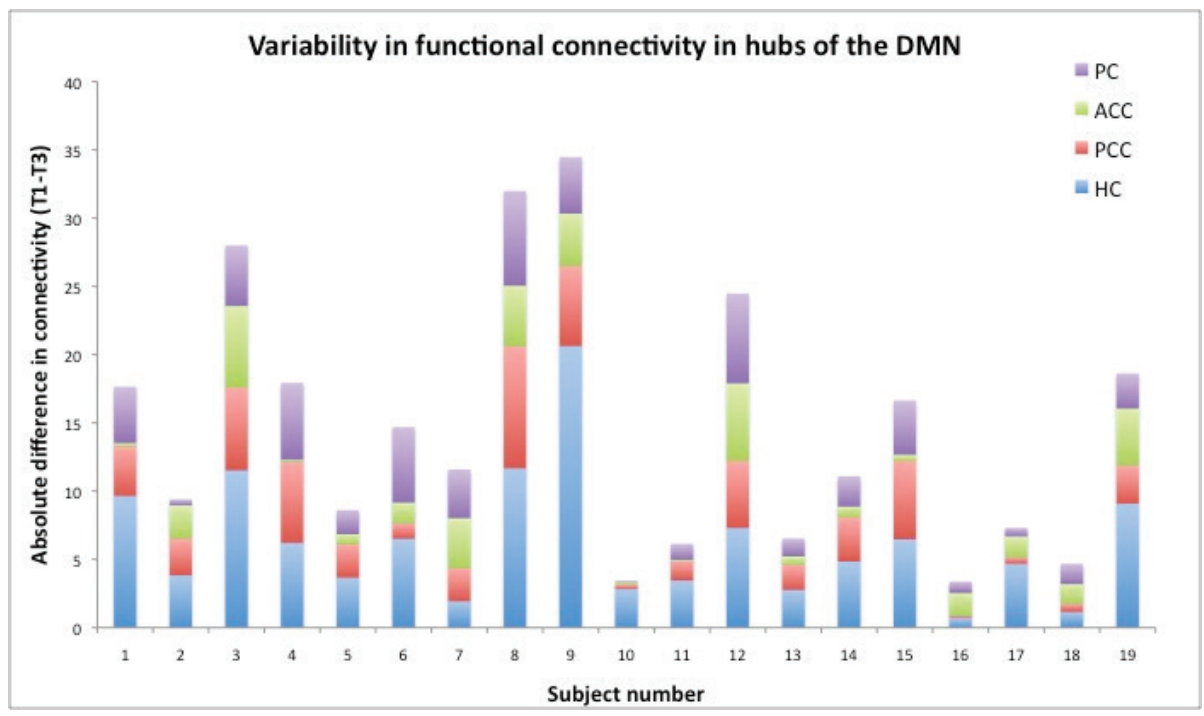

Figure 4. Subject-specific variability in functional connectivity in hubs of the DMN. Individual data-points per subjects are depicted on the $X$-axis whereas the length of the coloured rectangle corresponds with the degree of variability in a certain region of interest (longer rectangle $=$ more variability between diferent data-points). Absolute differences between the first and the last time point (scansession) are depicted on the $\mathrm{Y}$-axis. 

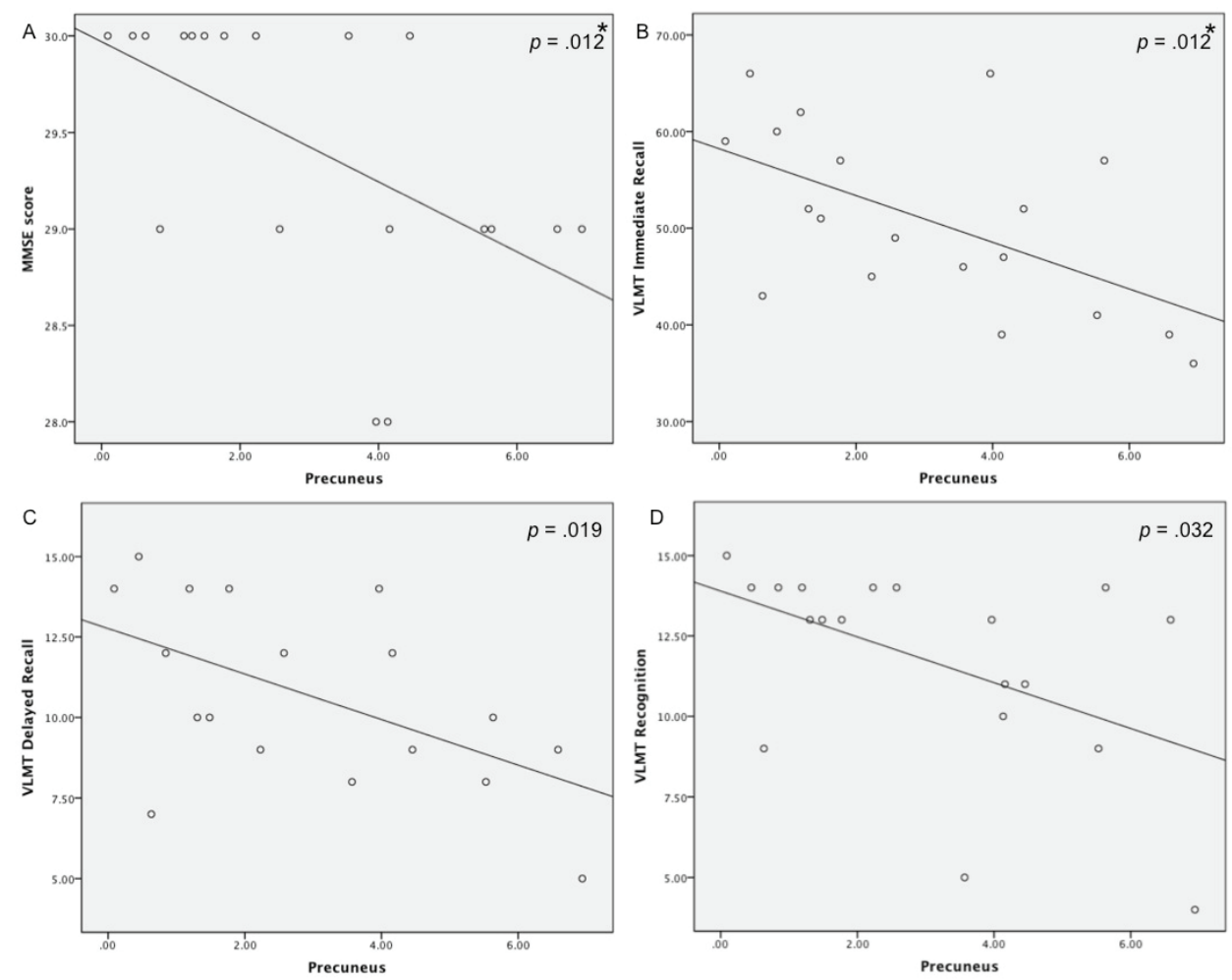

Figure 5. Associations between variability in functional connectivity in hubs of the default mode network and neuropsychological performance. (A) Higher variability in precuneal connectivity was associated with a decrease in MMSE score $(B=-0.18 \pm 0.06 ; p=0.012),(B)$ lower immediate recall scores $(B=-2.42 \pm 0.86 ; p=0.012)$, (C) lower delayed recall scores $(B=$ $-0.71 \pm 0.27 ; p=.019)$ and $(D)$ lower recognition scores $(B=-0.71 \pm 0.30 ; p=.032)$.

$* \mathrm{p}$-values significant after correction for multiple comparisons.

\section{DISCUSSION}

The present study evaluated the potential of RSNs as a biomarker for disease, by investigating its test-retest reliability in healthy aging. To the best of our knowledge, this is the first study assessing the stability of 8 RSNs over time in both $\mathrm{HO}$ and $\mathrm{HY}$ subjects by means of rs-fMRI. Thus far studies mainly focused on HO $[23,24]$ or HY subjects [25, 26], compared both groups cross-sectionally [27] or investigated the stability of resting state networks (RSNs) by means of other imaging modalities (e.g. PET) [28]. Test-retest reliability in the present study was investigated both on 
a voxel-wise basis and by means of intraclass correlation coefficients. Second, we correlated regional DMN instability in older individuals with neuropsychological test performance in order to identify people who might carry an increased risk for future cognitive impairment.

\section{Age-effects}

A decrease in FC with aging was found in the auditory system and the DMN. Even after correction for GM atrophy and education, decreases in $\mathrm{FC}$ in $\mathrm{HO}$ subjects remained significant, indicating that age-related changes in connectivity are not solely due to reductions in gray matter volume or cognitive reserve. Previous studies show similar results, viz., a negative association between age and FC of the DMN [29, 30]. Small clusters of increased FC in older subjects were found in the sensory-motor system. These differences were mainly found in the precentral gyrus which forms the posterior border of the frontal lobe, and could possibly be explained by the posterior-anterior shift in aging [31]. Normal aging seems to induce a connectivity disruption within the DMN, more specifically along the anterior-posterior axis of this network [32], showing reduced deactivations in occipitotemporal areas and increased deactivations in bilateral frontal areas (e.g. precentral gyrus) in $\mathrm{HO}$ compared to HY [33].

In line with our findings, an age-related FC reduction has been reported within superior and middle frontal PCC/precuneus, middle temporal, superior parietal, and medial areas such as medial prefrontal, anterior cingulate, and hippocampal regions [34]. On the other hand, older subjects were also found to show greater activity at rest, or greater deactivations, mainly in anterior brain areas. This increased activity at rest in frontal DMN regions of elderly adults has been interpreted as an attempt to compensate for the decrease of resting-state activity in posterior DMN areas [33].

\section{Stability of resting state networks over time}

No significant differences in FC over time were found in each of the eight investigated networks for the total group of healthy subjects. Furthermore, intraclass correlation coefficients between different time points mainly showed good to excellent reproducibility. Previous studies with a different design (e.g. one age group) report similar results, in particular in the DMN. One study in $\mathrm{HO}$ subjects showed a relative 
stability of rest-specific activity over time in regions commonly associated with the default-mode [28]. Other studies found that DMN coactivation patterns are reproducible in HY subjects (Meindl, et al., 2010; Shehzad, et al., 2009). The findings above indicate, in line with previous studies, that RSNs can be reliably reproduced over time.

\section{Relevance for early memory impairment}

As known from the literature, posterior parietal regions are important nodes for memory encoding and retrieval and show strong FC [35]. The vulnerability of these nodes for $A D$ pathology is related to their extensive connections with other $A D$ signature regions (e.g. hippocampus) and high metabolic activity compared to other regions of the DMN [36].

A number of studies found a reduction in connectivity between both the hippocampus $[37,38]$ and precuneus $[6,7,39-41]$ and several other brain regions in (amnestic) $\mathrm{MCl}$ or $\mathrm{AD}$. The present study found that in $\mathrm{HO}$ subjects, instability of FC between the precuneus and the rest of the DMN was linked with subtle alterations in global cognitive impairment and immediate recall, even after correction for multiple comparisons. This finding is of particular relevance since structural and functional alterations in precuneal regions are a consistent finding in various diseases such as $A D$. The precuneus, acting a hub, has extensive connections with other brain regions, increasing its sensitivity for metabolic and pathological influences. Abnormal connectivity patterns were also found within PCC regions in patients suffering from early $A D[42,43]$. In this study, however, no associations were found between instability of FC in hippocampal/posterior cingulate regions and neuropsychological test performance. Besides the fact that our study population comprises of healthy subjects, this finding could also be due to the fact that no spatial memory task was administered which would have possibly been more sensitive to subtle hippocampal or PCC dysfunction. Recent evidence pointed out that stable RSNs may represent healthy aging, whereas decreased RSN reliability may indicate progressive neurofunctional alterations before the actual manifestation of clinical symptoms [23]. In order to strengthen our conclusion that instability in precuneal connectivity is a precursor of cognitive problems, this hypothesis requires testing in subjects with subjective and/or mild cognitive impairment. Associations between other nodes of the DMN and neuropsychology may possibly become apparent in later stages of the disease process.

266 | Chapter 8: Stability of resting state networks in aging 


\section{Limitations and future perspectives}

The present study has several limitations. First, a predefined template was used instead of a data-driven independent component analysis (ICA) approach. It may be argued that predefined networks limit the scope of observations by restricting the criterion of connectivity to temporal similarity within a given network. These networks were however chosen on the basis of previous studies that had reproduced them in entirely different populations [14, 44]. An advantage is that typical ambiguities associated with ICA are avoided (e.g. specificity of the investigated group, model order). Second, older subjects with memory problems were not included in this study. Future studies could additionally include subjects with memory problems for discriminant analysis or longitudinally follow groups of healthy subjects in order to re-evaluate their cognitive status after a number of years. Third, it is known that certain mechanisms related to BOLD-variability might have an influence on BOLD-reactivity differences between old and young subjects [45]. One study found that there is substantial variability in responses collected across subjects and that differences between multiple scans sessions within a single subject seem to be less variable [46]. Another study suggests including an estimate of hemodynamic response function in order to solve some of the variability isssues across subjects [47]. It is also known that other factors such as caffeine intake [48], physiological factors [49] and sleep during scanning might have an influence on the BOLD-response [50]. In the present study, we did not control for caffeine intake nor for respiratory factors by means of external recordings of physiology. We however corrected for the influence of noise by using methods inherent to the ICA-approach, e.g. correction for head motion and low frequency artefacts. Regarding sleep and vigilance, we did not measure EEG-activity during $\mathrm{fMRI}$, which would have been the most sensitive technique. However, a questionnaire was administered in which subjects, after scanning, reported their sleepiness during scanning and whether they had fallen asleep. From the total group of subjects, over all scanning sessions, one subject fell asleep during one of the three scanning sessions. Fourth, stability was assessed at one-month interval. Neuronal changes due to the process of aging possibly require longer intervals. On the other hand, short intervals form the optimal paradigm for evaluation of the resting state technique and to validate FC measurements as such. Fifth, previous studies showed that resting-state FC changes overlap with structural connectivity alterations and that combining these modalities 
can enrich our understanding of underlying brain networks [51]. Future studies could include techniques such as diffusion tensor imaging to extensively investigate the process of healthy aging or disease, both on a functional and structural level. Finally, recent evidence points out that healthy subjects with amyloid plaque deposition in the brain (investigated by means of Positron Emission Tomography (PET)) show patterns of decreased FC at rest [52]. A recent study was unable to replicate these findings in diagnostic groups [53]. To investigate whether amyloid depositions precedes aberrant $\mathrm{FC}$ in the $\mathrm{DMN}$, and to explore the diagnostic value of each of these techniques, future studies could combine resting state modalities together with PET. A major strength of this study is the correction for gray matter atrophy and education. A large fraction of previous rs-fMRI studies did not correct for gray matter atrophy nor for cognitive reserve, which both play a prominent role in the process of aging. Furthermore, the present study used dual regression techniques to evaluate differences in FC between groups and time points. Hence, rater time is limited with the majority of the analysis being automated.

\section{Clinical implications}

The exploration of FC in RSNs by means of rs-fMRI has the advantage of being noninvasive, readily available, and does not require complex subject interaction [54]. This is essential in older patients and especially when working with patients suffering from AD. From a research perspective, RSNs can be used for the investigation of disease progression over time, and for monitoring of healthy older subjects. From a clinical point of view, RSNs can be used to select patients for clinical trials or to aid the early diagnostic process. A recent study found high diagnostic accuracy rates for the investigation of the DMN by means of rs-fMRI, ranging from $64-97 \%$, depending on the method of analysis used [55].

\section{CONCLUSION}

The present study provides important insights into the reproducibility of RSNs in healthy aging. Measurements of FC in RSNs, investigated by means of rs-fMRI were stable over different time points, showing good to excellent reproducibility, both in healthy old and healthy young subjects. Age-effects were identified, indicating a decrease in $\mathrm{FC}$ in the $\mathrm{DMN}$ and in the auditory system, associated with aging. Loss of stability of FC in vulnerable regions of the DMN (e.g. precuneus) could become a predictor for future memory problems on an individual level. 


\section{REFERENCES}

1. Fox MD, Snyder AZ, Vincent JL, Corbetta M, Van Essen DC, Raichle ME. The human brain is intrinsically organized into dynamic, anticorrelated functional networks. Proceedings of the National Academy of Sciences of the United States of America 2005;102:9673-9678.

2. Greicius MD, Krasnow B, Reiss AL, Menon V. Functional connectivity in the resting brain: A network analysis of the default mode hypothesis. Proceedings of the National Academy of Sciences 2003;100:253-258.

3. Buckner R, Snyder A, Shannon B, et al. Molecular, structural, and functional characterization of Alzheimer's disease: evidence for a relationship between default activity, amyloid, and memory. The Journal of neuroscience : the official journal of the Society for Neuroscience 2005;25:7709-7717.

4. Broyd SJ, Demanuele C, Debener S, Helps SK, James CJ, Sonuga-Barke EJS. Default-mode brain dysfunction in mental disorders: A systematic review. Neuroscience \& Biobehavioral Reviews 2009;33:279-296.

5. Tomasi D, Volkow ND. Aging and functional brain networks. Mol Psychiatry 2012;17:549-558.

6. Agosta F, Pievani M, Geroldi C, Copetti M, Frisoni GB, Filippi M. Resting state fMRI in Alzheimer's disease: beyond the default mode network. Neurobiology of Aging 2012;33:1564-1578.

7. Fleisher AS, Sherzai A, Taylor C, Langbaum JBS, Chen K, Buxton RB. Restingstate BOLD networks versus task-associated functional MRI for distinguishing Alzheimer's disease risk groups. Neurolmage 2009;47:1678-1690.

8. Folstein MF, Folstein SE, McHugh PR. "Mini-mental state": A practical method for grading the cognitive state of patients for the clinician. Journal of Psychiatric Research 1975;12:189-198.

9. Rey A. The clinical examination in psychology. Oxford, England: Presses Universitaries De France. 1964;1958:222.

10. Tombaugh TNTN. Trail Making test A and B: Normative Data Stratified by Age and Education. Archives of Clinical Neuropsychology : The Official Journal of the National Academy of Neuropsychologists 2004;19:203-214.

11. Meyers J, \& Meyers, KR. . Rey complex figure test and recognition trial: Professional manual. 1941.

12. Beckmann CF, DeLuca M, Devlin JT, Smith SM. Investigations into resting-state 
connectivity using independent component analysis. Philosophical Transactions of the Royal Society Biological Sciences 2005;360:1001-1013.

13. Beckmann C, Mackay C, Filippini N, Smith S. Group comparison of resting-state fMRI data using multi-subject ica and dual regression. Neurolmage 47 (Suppl 1) 2009;1.

14. Filippini N, Maclntosh BJ, Hough MG, et al. Distinct patterns of brain activity in young carriers of the APOE-e4 allele. Proceedings of the National Academy of Sciences 2009;106:7209-7214.

15. Smith SM, Nichols TE. Threshold-free cluster enhancement: Addressing problems of smoothing, threshold dependence and localisation in cluster inference. Neurolmage 2009;44:83-98.

16. Smith SM. Fast robust automated brain extraction. Human Brain Mapping 2002;17:143-155.

17. Burgmans S, van Boxtel MPJ, Smeets F, et al. Prefrontal cortex atrophy predicts dementia over a six-year period. Neurobiology of Aging 2009;30:1413-1419.

18. Clerx L, van Rossum I, Burns L, et al. Measurements of medial temporal lobe atrophy in the prediction of Alzheimer's Disease in subjects with $\mathrm{MCl}$. Neurobiology of Aging 2013.

19. Bartko JJ. Measurement and Reliability: Statistical Thinking Considerations. Schizophrenia Bulletin 1991;17:483-489.

20. Shrout PE, Fleiss JL. Intraclass correlations: uses in assessing rater reliability. Psychological Bulletin 1979;2:420-428.

21. Rosner B. Fundamentals of biostatistics. Belmont, CA: Duxbury Press, 2005.

22. Benjamini Y, Hochberg Y. Controlling the False Discovery Rate: A Practical and Powerful Approach to Multiple Testing. Journal of the Royal Statistical Society. Series B (Methodological) 1995;57:289-300.

23. Blautzik J, Keeser D, Berman A, et al. Long-Term Test-Retest Reliability of Resting-State Networks in Healthy Elderly Subjects and Patients with Amnestic Mild Cognitive Impairment. Journal of Alzheimer's Disease 2013;34:741-754.

24. Guo CC, Kurth F, Zhou J, et al. One-year test-retest reliability of intrinsic connectivity network fMRI in older adults. Neurolmage 2012;61:1471-1483.

25. Meindl T, Teipel S, Elmouden R, et al. Test-retest reproducibility of the defaultmode network in healthy individuals. Human Brain Mapping 2010;31:237-246.

26. Park B, Kim JI, Lee D, Jeong S, Lee JD, Park H. Are brain networks stable during a 24-hour period? Neurolmage 2012;59:456-466.

270 | Chapter 8: Stability of resting state networks in aging 
27. Koch W, Teipel S, Mueller S, et al. Effects of aging on default mode network activity in resting state fMRI: Does the method of analysis matter? Neurolmage 2010;51:280-287.

28. Beason-Held L, Kraut M, Resnick S. Stability of default-mode network activity in the aging brain. Brain Imaging Behav 2009;14:123-131.

29. Onoda K, Ishihara M, Yamaguchi S. Decreased Functional Connectivity by Aging Is Associated with Cognitive Decline. Journal of Cognitive Neuroscience 2012;24:2186-2198.

30. Hafkemeijer A, van der Grond J, Rombouts SARB. Imaging the default mode network in aging and dementia. Biochimica et Biophysica Acta (BBA) - Molecular Basis of Disease 2012;1822:431-441.

31. Mowinckel AM, Espeseth T, Westlye LT. Network-specific effects of age and in-scanner subject motion: A resting-state fMRI study of 238 healthy adults. Neurolmage 2012;63:1364-1373.

32. Mevel K, Landeau B, Fouquet M, et al. Age effect on the default mode network, inner thoughts, and cognitive abilities. Neurobiology of Aging 2012.

33. Davis SW, Dennis NA, Daselaar SM, Fleck MS, Cabeza R. Qué PASA? The Posterior-Anterior Shift in Aging. Cerebral Cortex 2008;18:1201-1209.

34. Mevel K, Chetelat G, Eustache F, Desgranges B. The Default Mode Network in Healthy Aging and Alzheimer's Disease. Int J Alzheimers Dis. 2011;2011.

35. Jacobs HIL, van Boxtel MPJ, Heinecke A, et al. Functional integration of parietal lobe activity in early Alzheimer disease. Neurology 2012;78.

36. Buckner RL, Sepulcre J, Talukdar T, et al. Cortical Hubs Revealed by Intrinsic Functional Connectivity: Mapping, Assessment of Stability, and Relation to Alzheimer's Disease. The Journal of Neuroscience 2009;29:1860-1873.

37. Zhou Y, Dougherty Jr JH, Hubner KF, Bai B, Cannon RL, Hutson RK. Abnormal connectivity in the posterior cingulate and hippocampus in early Alzheimer's disease and mild cognitive impairment. Alzheimer's and Dementia 2008;4:265270.

38. Allen $G$ BHMR, et al. REduced hippocampal functional connectivity in alzheimer disease. Archives of Neurology 2007;64:1482-1487.

39. Greicius MD, Srivastava G, Reiss AL, Menon V. Default-mode network activity distinguishes Alzheimer's disease from healthy aging: Evidence from functional MRI. Proceedings of the National Academy of Sciences of the United States of America 2004;101:4637-4642. 
40. Rombouts SARB, Barkhof F, Goekoop R, Stam CJ, Scheltens P. Altered resting state networks in mild cognitive impairment and mild Alzheimer's disease: An fMRI study. Human Brain Mapping 2005;26:231-239.

41. Sorg C, Riedl V, Mühlau M, et al. Selective changes of resting-state networks in individuals at risk for Alzheimer's disease. Proceedings of the National Academy of Sciences 2007;104:18760-18765.

42. Bai F, Zhang Z, Watson DR, et al. Absent gender differences of hippocampal atrophy in amnestic type mild cognitive impairment. Neuroscience Letters 2009;450:85-89.

43. Jacobs HIL, Radua J, Lückmann HC, Sack AT. Meta-analysis of functional network alterations in Alzheimer's disease: Toward a network biomarker. Neuroscience \& Biobehavioral Reviews 2013;37:753-765.

44. Damoiseaux JS, Rombouts SARB, Barkhof $F$, et al. Consistent resting-state networks across healthy subjects. Proceedings of the National Academy of Sciences 2006;103:13848-13853.

45. D'Esposito M, Zarahn E, Aguirre GK, Rypma B. The Effect of Normal Aging on the Coupling of Neural Activity to the Bold Hemodynamic Response. Neurolmage 1999;10:6-14.

46. Aguirre GK, Zarahn E, D'Esposito M. The Variability of Human, BOLD Hemodynamic Responses. Neurolmage 1998;8:360-369.

47. Handwerker DA, Ollinger JM, D'Esposito M. Variation of BOLD hemodynamic responses across subjects and brain regions and their effects on statistical analyses. Neurolmage 2004;21:1639-1651.

48. Diukova A, Ware J, Smith JE, et al. Separating neural and vascular effects of caffeine using simultaneous EEG-FMRI: Differential effects of caffeine on cognitive and sensorimotor brain responses. Neurolmage 2012;62:239-249.

49. Murphy K, Birn RM, Bandettini PA. Resting-state fMRI confounds and cleanup. Neurolmage 2013.

50. Olbrich S, Mulert C, Karch S, et al. EEG-vigilance and BOLD effect during simultaneous EEG/fMRI measurement. Neurolmage 2009;45:319-332.

51. Greicius MD, Supekar K, Menon V, Dougherty RF. Resting-State Functional Connectivity Reflects Structural Connectivity in the Default Mode Network. Cerebral Cortex 2009;19:72-78.

52. Sheline YI, Raichle ME. Resting State Functional Connectivity in Preclinical Alzheimer's Disease. Biological Psychiatry 2013.

272 | Chapter 8: Stability of resting state networks in aging 
53. Adriaanse SM, Sanz-Arigita EJ, Binnewijzend MAA, et al. Amyloid and its association with default network integrity in Alzheimer's disease. Human Brain Mapping 2012:n/a-n/a.

54. Greicius MD. Resting-state functional connectivity in neuropsychiatric disorders. Current Opinion in Neurology 2008;21:424-430.

55. Koch W, Teipel S, Mueller S, et al. Diagnostic power of default mode network resting state $\mathrm{fMRI}$ in the detection of Alzheimer's disease. Neurobiology of Aging 2012;33:466-478. 


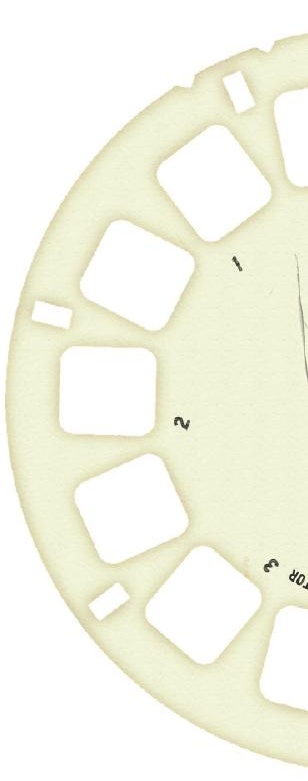




\section{Chapter 9 \\ General Discussion}

Future directions

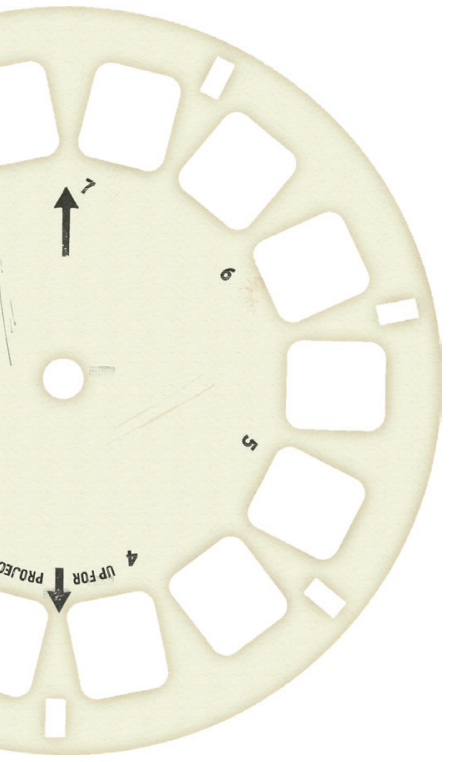




\section{Rationale}

The main objective of this thesis was to expand insight into the use of novel magnetic resonance imaging (MRI) techniques for the early detection, diagnosis and prognosis of Alzheimer's disease (AD). The clinical value of both conventional and novel imaging markers was evaluated for their possible contribution to patient management.

\section{Added value of MRI in clinical practice}

Current clinical practice in most Memory Clinics consists of a cognitive examination of patients by means of a neuropsychological test battery, together with a physical and neurological investigation, (hetero)anamnesis, a blood test and a brain scan. Based on the results of all these tests a diagnosis is made, treatment is initiated, and a follow-up trajectory is defined. Given the limited resources in health care, an efficient use of diagnostic tests with a minimum of costs per diagnosis together with a maximum accuracy is important. A first question that automatically arises from this clinical approach formed the starting point of the thesis: 'What is the added value of MRI to general diagnostics, and more specific: to neuropsychological test performance?'.

First of all, by means of MRI one can exclude other underlying diseases or possible causes of cognitive impairment [1, 2], and assess the presence of vascular (i.e. white matter lesions, lacunes, infarcts) and treatable diseases (i.e. subdural hematoma, tumors). Second, structural MR images allow an evaluation of atrophy in medial temporal lobe (MTL) regions, a key marker for $A D$, by means of a qualitative visual rating scale [3]. In most memory clinics, MRI investigation is actually limited to these assessments.

In Chapter 2 we found that MRI hippocampal volume was the best predictor of conversion from mild cognitive impairment $(\mathrm{MCl})$ to $A D$. Measuring hippocampal volume furthermore improved the accuracy over memory tests alone. In line with others was found that the delayed recall test is the best neuropsychological predictor for $A D$ in subjects with $\mathrm{MCl}$. We pointed out that when MRI-assessment is available, that adding a measurement of hippocampal volume to neuropsychological test performance is preferred. We also found that the added value of performing 
a visual rating scale on top of neuropsychological testing is limited in terms of sensitivity and specificity. Assessing disease progression by means of episodic memory tests (delayed recall) and MMSE-score is an alternative at the cost of a lower sensitivity and specificity. In line with the results in Chapter 3, we concluded that the likelihood to identify $\mathrm{MCl}$ patients at risk for $\mathrm{AD}$-conversion is highest for volumetric measurements. Assessment of hippocampal volume together with a delayed recall test may be sufficient for short-term prognostic purposes (e.g. follow-up of MCl-patients). Recent evidence pointed out that MTL atrophy is not completely specific for AD since it can also be found in other forms of dementia [4]. It is furthermore known that AD pathology is not restricted to the MTL. Chapter 4 therefore explored differences between healthy controls, $\mathrm{MCl}$ and $\mathrm{AD}$-patients in and outside the MTL. We evaluated if four techniques assessing gray matter (GM) atrophy differences in the brain show the same pattern of abnormalities throughout the same dataset. We found that the sensitivity of each method to detect group differences was region-specific. Manual volumetric measurements together with voxel based morphometry are preferred techniques for assessing GM differences showing abnormalities in most of the investigated regions, with a predominance of the MTL in the early phase. Automated FreeSurfer volumetric measurements show similar performances in the early phase, displaying group differences in the posterior parietal cortex (PPC) but not in MTL regions. Measurements of cortical thickness are less sensitive in the $\mathrm{MCl}$ stage and its sensitivity is restricted to the MTL and PPC regions in later stages of the disease (AD).

Taken together, the results of the studies described in chapters 2 to 4 allow us to draw three major conclusions. First, MRI has indeed an added value on top of neuropsychological assessment, both in the field of diagnosis and prognosis (disease tracking). Second, we confirmed the early involvement of the MTL (Chapter 2 to 4) and its clinical importance in predicting conversion to $A D$ in subjects with $\mathrm{MCl}$ (Chapter 2 and 3). We also showed that atrophy of the MTL is related to cognitive decline (Chapter 3). Third, our findings add to the existing body of knowledge that the diagnostic value of MR imaging in $A D$ is not limited to qualitative assessments in MTL regions (Chapter 4). Quantitative, volumetric measurements show higher predictive accuracy rates than visual rating scales (Chapter 3 ) and the sensitivity of volumetric markers for (early) AD is not restricted to MTL regions since also posterior parietal and prefrontal regions show macroscopic alterations throughout 
the disease process (Chapter 4). However, a major pitfall for implementing volumetric measurements in clinical practice is access to an infrastructure which allows quantitative assessments (see discussion below).

\section{Gray matter atrophy or white matter connectivity?}

Besides GM atrophy as a key hallmark of $A D$, white matter (WM) integrity is decreased in $\mathrm{MCl}$ and $\mathrm{AD}$ suggesting a potential diagnostic value that may possibly help to improve the diagnostic accuracy of GM atrophy measurements $[5,6]$. In Chapter 5 we evaluated the potential of DTI as an early marker for AD, in relation to MTL atrophy measurements, by using a meta-analysis approach. We concluded that in general, effect sizes of volumetric MTL atrophy measurements were equal or larger than effect sizes of DTI. However, in the early stages (controls vs. $\mathrm{MCl}$ ), hippocampal mean diffusivity showed larger effect sizes than hippocampal volume. Despite studies stating that DTI measurements seem to be more sensitive than volumetric measurements [7] our meta-analysis did not clearly support the notion that DTI is superior to structural MTL assessment in detecting early stage AD. In chapter 6 , we tried to replicate the findings of chapter 5 by means of a case-control study. Cross-sectionally, we found a constrained pattern of GM differences in left parahippocampal gyrus (healthy controls versus subjects with $\mathrm{MCl}$ and $\mathrm{AD}$ ) and left posterior cingulate regions (controls versus $A D$ ). This in contrast with previous findings that show a widespread pattern of GM atrophy in $A D$ and a more restricted GM atrophy pattern in $\mathrm{MCl}$ patients [8]. In addition, diffusivity metrics were more sensitive for group differences than atrophy measurements, and were less influenced by the effects of aging, gender and scan parameters in a priori selected ROIs. Diffusivity differences were found in all the investigated structures, whereas changes in absolute diffusivity (mean diffusivity (MD), radial diffusivity (DR) and axial diffusivity (DA)) were more pronounced than changes in anisotropy (FA).

Since longitudinal cognitive and behavioral data were available, we additionally investigated the value of these markers for predicting decline at 2 years followup. We found that diffusivity differences in the genu of the corpus callosum but not volumetric measures predicted decline in a group of $\mathrm{MCl}$-subjects. Important to note is that, in contrast to our meta-analysis in chapter 5, automated atrophy measurements (FreeSurfer) were evaluated here, both in and outside the MTL. The novelty of this study was that the pattern of gray and white matter alterations in both $\mathrm{MCl}$ and $\mathrm{AD}$-patients was investigated in a multicenter setting, and that 
diagnosis was based on expert panel judgements.

From the results in chapter 5 and 6 we can conclude that DTI is a sensitive method to detect white matter changes in subjects with $\mathrm{MCl}$ and $\mathrm{AD}$. In widespread brain regions diffusivity is already impaired early in the disease process, with more severe white matter disruptions with increasing disease severity. Diffusivity measurements show higher effect sizes than volumetric measurements in discriminating healthy controls from $\mathrm{MCl}$ and $\mathrm{AD}$ and are less influenced by the effects of aging, gender and center in apriori selected ROIs. DTI metrics in medial temporal, posterior parietal and callosal regions may therefore serve as an early marker for degeneration, even before atrophy is detectable. Diffusivity metrics in the genu of the corpus callosum can predict decline in a group of $\mathrm{MCl}$-subjects.

\section{Robustness of structural atrophy and functional connectivity measurements}

In the search for new imaging markers for disease, we must be well aware that techniques may be influenced by internal (e.g. patients age) and external (e.g. software, hardware) factors. In order to explore some of these influences, we carried out a number of methodological evaluations in the third and last part of the thesis.

Automated segmentation techniques become more and more popular nowadays due to the time-intensive nature of manual volumetry. The question therefore arises if processing conditions such as workstation type, operating system and software version affect automated segmentation results and thus influence group differences. Chapter 7 investigated the robustness of FreeSurfer to capture morphological changes in the brain against varying processing variables and in comparison to manual measurements. We concluded that later versions of FreeSurfer were more accurate than earlier versions, especially in medial temporal and posterior parietal regions. A lot of research in the last decade has pointed out resting state functional connectivity as a marker for AD [9]. The first step toward a biomarker is assuring that age-effects do not influence the reliability of RSNs. Chapter 8 investigated whether functional connectivity patterns can be reliably reproduced over time in healthy old and young subjects. It was found that measurements of functional connectivity, investigated by means of resting state functional MRI were stable over different time points, showing good to excellent reproducibility, both in healthy old 
and healthy young subjects. Age-effects were furthermore identified, indicating a decrease in FC in the DMN and in the auditory system, associated with aging.

From the findings in chapter 7 and 8 we can conclude that both FreeSurfer automated volumetry and resting state functional connectivity are reliable measurements to study the process of aging and disease. Prior work of our group has shown that mixing up versions, workstations or operating systems might lead to significant differences in cortical thickness or volumetry in schizophrenic patients [10]. The findings in chapter 7 were much more positive, but we however agree with the software developers that it is advised to keep the research environment stable. The necessity to control for age-effects in dementia research is furthermore quite evident as age might cover or lead to an overestimation of group-specific differences [11].

\section{Underlying mechanisms of gray and white matter pathology in AD}

Alterations in gray and white matter are independent pathological mechanisms in $A D$ [12-14]. GM atrophy essentially results from neurodegeneration by the accumulation of aggregated hyperphosphorylated tau protein ('tangles'), deposition of amyloid-beta ('plaques'), oligodendrocyte death and reactive gliosis $[15,16]$. The exact neurobiological substrate of diffusion changes still remains unresolved. Alterations of anisotropy (FA) have been suggested to correlate primarily with the integrity of the axonal membrane [17]. An increase in mean diffusivity (MD) is thought to reflect enlargement in the extracellular space due to altered cytoarchitecture (i.e. loss of neurons, axons, and dendrites), suggesting immaturity or degeneration of the tissue leading to elevated water diffusivity within these regions $[18,19]$. Intra-axonal structures are thought to determine the diffusion parallel to the axonal fibers (DA) [20], whereas the changes in myelin sheats and axonal membranes underline diffusion perpendicular to the axonal fibers (DR) [21]. In Chapter 6 was found that the discriminative value of diffusivity metrics is higher than that of GM atrophy suggesting that in most regions microstructural changes in WM precede macrostructural atrophy. The pattern of decreased WM integrity in Chapter 6 was consistent with the retrogenesis model of WM degeneration in $A D$, which shows that myelin breakdown follows a pattern that is the reverse of myelogenesis, thus first affecting late-myelinating fibers [22-24]. This retrogenesis model of WM change is furthermore consistent 
with the pattern of functional changes in early $A D$ where memory, language and executive problems are present in the absence of sensory and motor problems [25]. Apart from retrogenesis, also Wallerian degeneration plays a role in WM pathology in AD. In the Wallerian degeneration model, degradation of microstructure occurs secondary to GM pathology [26, 27]. The genu, splenium and cingulum fiber pathways investigated in Chapter 6 all share connections to MTL structures (e.g. hippocampus) known to be affected early in the course of AD [28] and changes in these structures (e.g. atrophy) could lead to Wallerian degeneration [29]. The results of Chapter 6 did not change when analyses were corrected for GM volume, suggesting that WM diffusivity differences were independent of GM atrophy patterns and that Wallerian degeneration is not the primary mechanism of WM change in AD. The lack of a significant correlation between diffusivity and volumetric measurements in regions outside the hippocampus strengthens the idea that DTI and MR-based volumetry measure different aspects of underlying pathology [30]. Since the data in chapter 6 was mainly cross-sectional we cannot adjudicate about causality. However, our findings suggest that WM changes in $A D$ are primary related to alterations in the integrity of myelin[21] rather than to Wallerian degeneration secondary to distal GM atrophy. These findings are in line with previous work $[24,31]$ and neuropathological evidence indicating that WM changes in $A D$ are not solely due to Wallerian degeneration [32].

\section{Scientific implications}

The results described in this thesis have several implications for future studies into the pathogenesis of early AD. In line with other studies, we showed that both GM atrophy and WM diffusivity play an important role in discriminating between healthy controls and (early) AD, predicting AD-conversion in subjects with $\mathrm{MCl}$ or predicting decline after 2 years [13, 33-37]. Associations with genetic, CSF and neuropsychological markers were investigated and techniques were validated both in single center and multi-center settings.

\section{Relation with other (non-imaging) AD-biomarkers}

In chapter 3 we found, in line with the literature, that hippocampal volume significantly correlated with CSF t-tau and p-tau but not with Aß1-42 [38-40]. These findings may be explained by the obervation that antemortem hippocampal volume significantly correlates with the density of neurofibrillary tangles at autopsy $[41,42]$ 
but not with amyloid beta plaque load [41]. The qualitative MTA-score and lateral ventricle volume correlated with CSF A $\beta 1-42$. This correlation may indicate that these MTL measures in part reflect generalised brain atrophy as previous studies showed that lower $A \beta 1-42$ levels but not $t$-tau levels were associated with total brain atrophy and ventricular volume [43]. Automated hippocampal volume at baseline was furthermore associated with APOE-e4 allele status in chapter 3.

\section{Multi-center effects}

Besides the above mentioned differences in software and hardware during preprocessing and analysis, also scanners (e.g. manufacturer, type, field strength) or scanparameters often vary in multi-center studies and may influence imaging results or group differences. Since the data used in chapter 3 consisted of scanners with different field strengths (1T and 1.5T), we investigated the possible effects of these differences on hippocampal volume measurements. First, we identified a study from one of our co-authors comparing hippocampal volumes of subjects scanned at both 1.5T and 3T scanners (Wolz 2013, in press). Hippocampal volume was measured by means of the LEAP method using data from the ADNI study. The correlation between the measurements on each type of scanner was very high $(r=0.98)$. The volumes measured on 3T were on average $24.4 \mathrm{~mm}^{3}$ or $1.17 \%$ larger than on 1.5T. Important to note is that the volume was also about $1.5 \%$ larger if the hippocampus was measured the second time on a scanner with the same magnetic field strength (either 1.5T or 3T; Wolz 2013, in press). This indicates that for the LEAP method, even a difference of 1.5T in field strength has no significant impact on hippocampal volume. Secondly, we tested the effect of field strength on the volumetric measures. We compared the volumes of 127 subjects scanned on a $1 T$ scanner with that of 201 subjects scanned on a 1.5T scanner with correction for age, gender, educational level, baseline MMSE score and follow-up diagnosis. The difference in volume between $1 \mathrm{~T}$ and $1.5 \mathrm{~T}$ scanners was only $0.2 \%$ for the LEAP method ( $p$-value $=0.9[44]), 0.9 \%$ for manual hippocampal volume ( $p$-value $=0.6)$, and $1.5 \%$ for the lateral ventricle volume ( $p$-value $=0.8$ ). Third, we tested whether field strength modulated the effect of the volumetric measures on conversion to AD-type dementia. The interaction between field strength and volumetric measure was not statistically significant for any of the volumetric measures $(p>0.15)$. These data indicate that the difference in field strength had no effect on hippocampal or ventricular volume. 
In chapter 7, we used multi-center DTI-data consisting of different scanners (manufacturers) and scan parameters with equal field strength (3T). One healthy volunteer ( 25 years old, male) was scanned twice at all centers to determine intra and inter center differences in DTI-values. In the hippocampal region we found variability values between $0.79-3.88 \%$ (depending on the DTI parameter) compared to $0.22-9.58 \%$ in the posterior cingulum. As expected, inter center variability was higher than intra center variability, and also region-dependent (hippocampus 2.07$6.85 \%$ vs. posterior cingulum $7.12-16.45 \%$ ). Our results are in line with recent evidence which reported a variation in FA across multiple scanners/field strengths both using a physical phantom and human brain scans [45].

The findings above indicate the importance of harmonization in the field of scanners, scan protocols, software versions, pre-processing steps and methods of analysis. These premonitions are not limited to the field of imaging since other markers (e.g. CSF, neuropsychology) also require standardisation of methods and validation of cut-offs. It is virtually impossible to correct for all sources and types of errors in research practice. It is however essential that we are aware of potential sources of error, that research protocols and analyses account for these factors and that corrections are performed, if possible.

\section{MRI in clinical practice: necessity or option?}

At this point, we are back to where we started in the beginning of this chapter: 'What is the role of MRI in clinical practice?' and 'Is MRI investigation necessary in clinical practice or does it only lead to additional burden from a patient's point of view?'. Taken together all three parts of the thesis, and building upon the existing body of knowledge, we can conclude that MRI plays an important role both for diagnostic and prognostic purposes. However, despite the clinical relevance of certain imaging modalities (e.g. hippocampal volumetry, connectivity), many of these findings are not readily transferrable to clinical practice. Translation to clinical settings remains to be demonstrated due to restrictions inherent to the technique (e.g. no standardization, no longitudinal validation, no availability of cut-offs, no expertise), or due to the unavailability of software or hardware amenities. 
For implementation in clinical practice, a marker requires that its dynamics are known at different disease stages and that its relationship with other imaging and biological markers is understood [46]. In chapter 3 was found that the predictive accuracy of both volumetric hippocampal measurements for AD-type dementia (manual and LEAP) was higher than that of the qualitative rating and lateral ventricle measure. Both measures of hippocampal volume significantly correlated with CSF t-tau and $p$-tau but not with A $\beta 1$-42. LEAP hippocampal volume at baseline was furthermore associated with APOE-e4 allele status (lower LEAP volumes in APOE-e4 carriers compared to APOE-e4 non-carriers). For the limited rater time (four minutes control time on a standard computer), LEAP automated hippocampal measurement may be preferred for clinical implementation.

A lot of effort has been made to point out the best biomarker (or combination of markers) for diagnosing early AD [47]. To date, indirect evidence has indicated that amyloid markers (assessed through PET-imaging or CSF) show more substantial abnormalities than structural $\mathrm{MRI}$ in the asymptomatic to $\mathrm{MCl}$ stage, and that CSF-levels are overall more sensitive as diagnostic tool [48]. Atrophy markers on the other hand are more sensitive to change (disease tracking) than CSF-markers $[49,50]$. However, a lot of inconsistencies are found regarding the initiator and accelerator roles of certain pathological events and thus markers for different disease stages $[48,51,52]$. According to recent findings one might consider all CSF and neuroimaging biomarkers (together with the presence of cognitive impairment) to the same degree whereas the presence of each factor increases the risk of underlying AD pathology [53]. Despite the high diagnostic accuracy rates of CSF biomarkers, lumbar puncture is feared by many patients due to the risk of severe complications and post-LP heaches in 2-4 percent of cases [54]. From a patients perspective, an MRI scan is a more feasible and less intrusive diagnostic alternative in clinical practice.

\section{Future research directions}

The restrictions mentioned above should be thoroughly investigated and resolved before quantitative measurements (structural atrophy or connectivity) can be succesfully implemented in everyday clinical practice. In order to implement 'MRIbeyond-MTA' as a biomarker for AD, future studies need to standardise automated techniques for use in clinical practice, and establish and validate cut-offs in studies with large sample sizes. Such cut-offs are needed to achieve the ultimate goal of 
an MRI-biomarker, namely a reliable diagnosis on a subject-level based on a single cross-sectional MRI scan.

Future studies need to assess the clinical relevance of the above mentioned techniques in large-scale clinical trials which aim to examine the diagnostic and prognostic value of certain techniques. Furthermore, treatment effects need to be mapped with different multimodal imaging modalities. The latter is even more important if disease modifying treatments become available that require easy accessible, cost-effective and non-invasive techniques to diagnose $A D$ in its earliest stages.

\section{A necessary stopover... to know or not to know?}

The research area of early biomarkers for $A D$ is highly motivated by the on-going development of disease-modifying treatment. However, to date there is no cure available for $A D$ yet, so there is no direct benefit from an early diagnosis. The question therefore arises if preclinical persons need to be informed about their status in clinical routine?

In short, early diagnosis may also negatively affect health and daily life of an earlystage AD patient. A few of the possible implications are feelings of hopelessness and despair which may lead to depression, loss of job and/or driver's license, and insurance issues. Besides an impact on the patient level, also the social system (relatives, employer) is concerned. On the contrary, even without treatment options, early diagnosis might be clarifying for the patient, facilitate access to tools which can help to cope with the progressive decline and enhance assistance from the health care system.

Extended use of early diagnostic tools in research environments is furthermore essential since these developments may lead to increased enrollment of patients in clinical trials which can in turn lead to novel treatment approaches.

\section{Conclusion}

In conclusion, this thesis expands insights into the added value of imaging markers as diagnostic tool for the early detection of AD. Atrophy of MTL structures is a candidate marker to assess change from $\mathrm{MCl}$ to moderate $A D$ stages (prognosis). 
We showed that relatively novel and thus less validated MRI markers such as DTI and resting state functional MRI are promising for diagnosing $A D$ in an early stage but need to be evaluated empirically. Standardization of operating procedures (for e.g. volumetric and connectivity approaches) will further consolidate our understanding of AD-pathology, facilitate comparison and integration of results, enable the establishment of cut-offs and consequently pave the way to clinical implementation. 


\section{REFERENCES}

1. Benzinger TLS. Radiologic approach to Alzheimer's disease and other dementias: The emerging role of diffusion tensor magnetic resonance imaging. Applied Radiology 2005;34:25-33.

2. Devanand DP, Liu X, Brown PJ, Huey ED, Stern Y, Pelton GH. A Two-Study Comparison of Clinical and MRI Markers of Transition from Mild Cognitive Impairment to Alzheimer's Disease. Int J Alzheimers Dis. 2012;2012.

3. Scheltens $P$, Leys $D$, Barkhof $F$, et al. Atrophy of medial temporal lobes on MRI in "probable" Alzheimer's disease and normal ageing: diagnostic value and neuropsychological correlates. J Neurol Neurosurg Psychiatry 1992;55:967972.

4. Barber RM, Gholkar AF, Scheltens PM, Ballard CM, McKeith IGM, O'Brien JTD. Medial temporal lobe atrophy on MRI in dementia with Lewy bodies. Neurology 1999;52:1153-1158.

5. Medina $\mathrm{D}$, deToledo-Morrell $\mathrm{L}$, Urresta $\mathrm{F}$, et al. White matter changes in mild cognitive impairment and AD: A diffusion tensor imaging study. Neurobiology of Aging 2006;27:663-672.

6. Wang L, Goldstein FC, Veledar E, et al. Alterations in Cortical Thickness and White Matter Integrity in Mild Cognitive Impairment Measured by WholeBrain Cortical Thickness Mapping and Diffusion Tensor Imaging. AJNR. American Journal of Neuroradiology 2009;30:893-899.

7. Müller MJ, Greverus D, Weibrich C, et al. Diagnostic utility of hippocampal size and mean diffusivity in amnestic $\mathrm{MCl}$. Neurobiology of Aging 2007;28:398403.

8. Gili $\mathrm{T}$, Cercignani $\mathrm{M}$, Serra $\mathrm{L}$, et al. Regional brain atrophy and functional disconnection across Alzheimer's disease evolution. Journal of Neurology, Neurosurgery \& Psychiatry 2011;82:58-66.

9. Adriaanse SM, Sanz-Arigita EJ, Binnewijzend MAA, et al. Amyloid and its association with default network integrity in Alzheimer's disease. Human Brain Mapping 2014 Mar;35(3).

10. Gronenschild EHBM, Habets P, Jacobs HIL, et al. The Effects of FreeSurfer Version, Workstation Type, and Macintosh Operating System Version on Anatomical Volume and Cortical Thickness Measurements. PLoS ONE 2012;7:e38234. 
11. Dukart J, Schroeter ML, Mueller K, the Alzheimer's Disease Neuroimaging I. Age Correction in Dementia - Matching to a Healthy Brain. PLoS ONE 2011;6.

12. Jacobs HIL, Van Boxtel MP, Gronenschild E, et al. Patterns of gray and white matter changes in individuals at risk for Alzheimer's disease. Curr Alzheimer Res 2012;9:1097-1105.

13. Rose SE, PhD ALJ, Chalk JB. Gray and white matter changes in Alzheimer's disease: A diffusion tensor imaging study. Journal of Magnetic Resonance Imaging 2008;27:20-26.

14. Salat DH, Greve DN, Pacheco JL, et al. Regional white matter volume differences in nondemented aging and Alzheimer's disease. Neurolmage 2009;44:1247-1258.

15. Braak H, Braak E, Bohl J. Staging of Alzheimer-related cortical destruction. Eur Neurol. 1993;33:403-408.

16. Jellinger KA, Bancher C. AD neuropathology. Neurology 1996;46:1186-1187.

17. Beaulieu C. The basis of anisotropic water diffusion in the nervous system -a technical review. NMR in Biomedicine 2002;15:435-455.

18. Sykova E. Extrasynaptic volume transmission and diffusion parameters of the extracellular space. Neuroscience 2004;129:861-876.

19. Kantarci KM, Petersen RCMDP, Boeve BFM, et al. DWI predicts future progression to Alzheimer disease in amnestic mild cognitive impairment. Neurology 2005;64:902-904.

20. Budde MD, Xie M, Cross AH, Song S-K. Axial Diffusivity Is the Primary Correlate of Axonal Injury in the Experimental Autoimmune Encephalomyelitis Spinal Cord: A Quantitative Pixelwise Analysis. The Journal of Neuroscience 2009;29:2805-2813.

21. Song S-K, Yoshino J, Le TQ, et al. Demyelination increases radial diffusivity in corpus callosum of mouse brain. Neurolmage 2005;26:132-140.

22. Ashford JW, Bayley PJ. Retrogenesis: A Model of Dementia Progression in Alzheimer's Disease Related to Neuroplasticity. Journal of Alzheimer's Disease 2013;33:1191-1193.

23. Reisberg B, Franssen EH, Souren LEM, Auer SR, Akram I, Kenowsky S. Evidence and mechanisms of retrogenesis in Alzheimer's and other dementias: Management and treatment import. American Journal of Alzheimer's Disease and Other Dementias 2002;17:202-212.

24. Stricker NH, Schweinsburg BC, Delano-Wood L, et al. Decreased white matter 
integrity in late-myelinating fiber pathways in Alzheimer's disease supports retrogenesis. Neurolmage 2009;45:10-16.

25. Becker JT, Huff F, Nebes RD, Holland A, Boller F. Neuropsychological function in Alzheimer's disease: Pattern of impairment and rates of progression. Archives of Neurology 1988;45:263-268.

26. Englund E. Neuropathology of white matter changes in Alzheimer's disease and vascular dementia. Dement Geriatr Cogn Disord. 1998;9:6-12.

27. Englund $E$, Brun A. White matter changes in dementia of Alzheimer's type: the difference in vulnerability between cell compartments. Histopathology 1990;16:433-439.

28. Braak H, Braak E. Evolution of the neuropathology of Alzheimer's disease. Acta Neurol Scand Suppl. 1996;165:3-12.

29. Coleman M. Axon degeneration mechanisms: commonality amid diversity. Nat Rev Neurosci. 2005;6:889-898.

30. Müller MJ, Greverus D, Dellani PR, et al. Functional implications of hippocampal volume and diffusivity in mild cognitive impairment. Neurolmage 2005;28:1033-1042.

31. Di Paola M, Di lulio F, Cherubini A, et al. When, where, and how the corpus callosum changes in $\mathrm{MCl}$ and $\mathrm{AD}$ : a multimodal MRI study. Neurology 2010;74:1136-1142.

32. Brun A, Englund E. A white matter disorder in dementia of the Alzheimer type: a pathoanatomical study. Ann Neurol. 1986;19:253-262.

33. Liu Y, Spulber G, LehtimV§ki KK, et al. Diffusion tensor imaging and TractBased Spatial Statistics in Alzheimer's disease and mild cognitive impairment. Neurobiology of Aging 2011;32:1558-1571.

34. Morey RA, Petty $\mathrm{CM}, \mathrm{Xu}$ Y, et al. A comparison of automated segmentation and manual tracing for quantifying hippocampal and amygdala volumes. Neurolmage 2009;45:855-866.

35. Serra L, Cercignani M, Lenzi D, et al. Grey and White Matter Changes at Different Stages of Alzheimer's Disease. Journal of Alzheimer's Disease 2010;19:147-159.

36. Teipel SJ, Sabri O, Grothe M, et al. Perspectives for Multimodal Neurochemical and Imaging Biomarkers in Alzheimer's Disease. Journal of Alzheimer's Disease 2013;33:S329-S347.

37. Teipel SJ WM, Meindl T, Frisoni G, Bokde AL, Fellgiebel A, Filippi M, Hampel Chapter 9: General Discussion | 289 
H, Klöppel S, Hauenstein K, Ewers M; EDSD study group. Anatomical MRI and DTI in the diagnosis of Alzheimer's disease: a European multicenter study. J Alzheimers Dis. 2012;31:S33-47.

38. Apostolova LG, Hwang KS, Andrawis JP, et al. 3D PIB and CSF biomarker associations with hippocampal atrophy in ADNI subjects. Neurobiology of Aging 2010;31:1284-1303.

39. Carmichael O, Xie J, Fletcher E, Singh B, DeCarli C. Localized hippocampus measures are associated with Alzheimer pathology and cognition independent of total hippocampal volume. Neurobiology of Aging 2012;33:1124-1141.

40. de Souza LC, Chupin M, Lamari F, et al. CSF tau markers are correlated with hippocampal volume in Alzheimer's disease. Neurobiology of Aging 2011.

41. Csernansky JGMD, Hamstra JBS, Wang LP, et al. Correlations Between Antemortem Hippocampal Volume and Postmortem Neuropathology in AD Subjects. Alzheimer Disease \& Associated Disorders 2004;18:190-195.

42. Jack J, C.R., Dickson DW, Parisi JE, et al. Antemortem MRI Findings Correlate with Hippocampal Neuropathology in Typical Aging and Dementia. Neurology 2002;58:750-757.

43. Wahlund L-O, Blennow K. Cerebrospinal fluid biomarkers for disease stage and intensity in cognitively impaired patients. Neuroscience Letters 2003;339:99102.

44. van Rossum IA, Vos SJ, Burns L, et al. Injury markers predict time to dementia in subjects with $\mathrm{MCl}$ and amyloid pathology. Neurology 2012;79:1801-1816.

45. Teipel SJ, Reuter S, Stieltjes B, et al. Multicenter stability of diffusion tensor imaging measures: A European clinical and physical phantom study. Psychiatry Research: Neuroimaging 2011;194:363-371.

46. Frisoni GB, Fox NC, Jack CR, Scheltens P, Thompson PM. The clinical use of structural MRI in Alzheimer disease. Nat Rev Neurol 2010;6:67-77.

47. Pike KE, Savage G, Villemagne VL, et al. Beta-amyloid imaging and memory in non-demented individuals: evidence for preclinical Alzheimer's disease. Brain 2007;130:2837-2844.

48. Jack CR, Knopman DS, Jagust WJ, et al. Hypothetical model of dynamic biomarkers of the Alzheimer's pathological cascade. The Lancet Neurology 2010;9:119-128.

49. Jack CR, Lowe VJ, Weigand SD, et al. Serial PIB and MRI in normal, mild cognitive impairment and Alzheimer's disease: implications for sequence of 
pathological events in Alzheimer's disease. Brain 2009;132:1355-1365.

50. Sluimer JD, van der Flier WM, Karas GB, et al. Whole-Brain Atrophy Rate and Cognitive Decline: Longitudinal MR Study of Memory Clinic Patients1. Radiology 2008;248:590-598.

51. Jack Jr CR, Knopman DS, Jagust WJ, et al. Tracking pathophysiological processes in Alzheimer's disease: an updated hypothetical model of dynamic biomarkers. The Lancet Neurology 2013;12:207-216.

52. Knopman DS, Jack CR, Wiste $\mathrm{HJ}$, et al. Brain injury biomarkers are not dependent on B-amyloid in normal elderly. Ann Neurol. 2012;73:1531-8249.

53. Chetelat G. Alzheimer disease: A[beta]-independent processes - rethinking preclinical AD. Nat Rev Neurol 2013;9:123-124.

54. Andreasen N, Minthon L, Davidsson P, et al. EValuation of csf-tau and csfabeta 1-42 as diagnostic markers for alzheimer disease in clinical practice. Archives of Neurology 2001;58:373-379. 


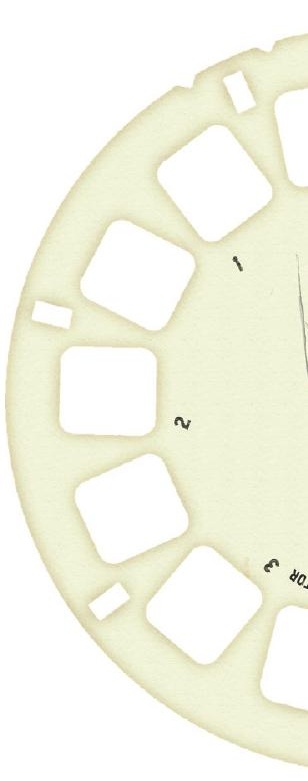


Appendix

Summary

Nederlandse samenvatting

Curriculum vitae

List of publications

Dankwoord

$\uparrow$

cs

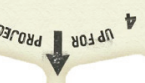





\section{SUMMARY}

Worldwide, 115 million people are at the forefront of an Alzheimer's tsunami. The baby boom generation is now entering their mid-sixties and many of them will suffer from Alzheimer's disease (AD), which now already affects 1 in every 8 people over age 65. Early diagnosis and treatment may help to reduce both patient and health-cost burden. Before clinical or cognitive deficits become apparent, a distinct set of underlying brain abnormalities such as a decrease in cerebral connectivity are present. Early detection of these (often subtle) abnormalities is therefore an important public health goal. The research described in this thesis aimed to expand insight into the use of both conventional (e.g. hippocampal volumetry) and novel (e.g. diffusion tensor imaging) magnetic resonance imaging (MRI) markers for early detection, diagnosis and prognosis of AD. MRI provides more information than macrostructural atrophy and vascular abnormalities. However, the added value and exact position of novel imaging techniques in the diagnostic field of AD was still unclear. The principle question of the thesis was whether MRI investigation is necessary in the diagnostic process of $A D$ and which MRI measurements can incrementally contribute to the early and specific diagnosis of Alzheimer disease.

The clinical value of novel imaging markers for $A D$ was evaluated in relation to current (gold standard) methods (i.e. hippocampal volumetry, visual rating scale). In the first part of the thesis (Chapter 2 to 4 ) we evaluated well-known imaging techniques which assess gray matter atrophy in the brain. In the second part (Chapter 5-6) we examined the discriminative and predictive value of a novel imaging marker for $A D$ : Diffusion Tensor Imaging (DTI). In the third part (Chapter 7-8) we investigated the robustness of structural and functional abnormalities in AD.

In chapter 2 we investigated in a multicenter setting $(n=184)$ if MR imaging markers increased predictive accuracy for $A D$ relative to a model which only included demographical and neuropsychological information. We found that hippocampal volume is the best predictor of conversion from mild cognitive impairment (MCl) to AD. Measuring hippocampal volume furthermore improved the accuracy over memory tests alone. In line with others was found that the delayed recall test is the best neuropsychological predictor for $A D$ in subjects with $\mathrm{MCl}$. We concluded that when MRI-assessment is available, that adding a measurement of hippocampal 
volume to neuropsychological test performance is preferred. Additionaly, it was found that the added value of performing a visual rating scale on top of neuropsychological testing is limited in terms of sensitivity and specificity. Since the MTL still receives a lot of attention in diagnosing $A D$, discriminating converters from non-converters and $A D$ from non- $A D$, chapter 3 compared the predictive value of 4 different MTL measurements in a multicenter $(n=328)$ and single-center setting ( $n=172$ ). Results showed that volumetric hippocampal measurements are the best predictors of conversion to AD-type dementia in subjects with $\mathrm{MCl}$ after 2 years follow-up (manual and LEAP hippocampal volume). Both volumetric measurements strongly correlate with cerebrospinal fluid (CSF) markers of neuronal injury (CSF $\mathrm{t}$-tau and $\mathrm{p}$-tau), are able to predict cognitive decline, and show consistent cutoff values between different cohorts. LEAP hippocampal volume has the advantage over manual volumetry that it needs much less rater time and shows no interrater variability effects. In addition, LEAP has a low technical failure rate.

Recent evidence pointed out that MTL atrophy is not completely specific for AD since it can also be found in other forms of dementia. It is furthermore known that AD pathology is not restricted to the MTL. Since the contribution of brain areas outside the MTL has become more evident, in chapter 4 we evaluated the discriminative value of several techniques measuring $G M$ atrophy in the brain, both in and outside MTL regions. It was investigated if four techniques assessing gray matter (GM) atrophy differences in the brain show the same pattern of abnormalities throughout the same dataset. We found that the sensitivity of each method to detect group differences was region-specific. Results showed that manual volumetric measurements together with voxel based morphometry are preferred techniques for assessing GM differences showing abnormalities in most of the investigated regions, with a predominance of the MTL in the early phase. Automated FreeSurfer volumetric measurements showed similar performances in the early phase, displaying group differences in the posterior parietal cortex but not in MTL regions. Measurements of cortical thickness are less sensitive in the $\mathrm{MCl}$ stage and its sensitivity is restricted to the MTL and PPC regions in later stages of the disease (AD).

Chapter 5 describes a meta-analysis which was conducted to evaluate the discriminative value of DTI in a group of controls subjects, patients with $\mathrm{MCl}$ and AD-pa- 
tients. We included seventy-six studies on MTL atrophy including 8122 subjects and fifty-five DTI studies including 2791 subjects. Effect sizes (ES) were calculated for several regions of interest known to be altered by pathophysiological mechanisms of $A D$, and were compared with ES of medial temporal lobe measurements. Results showed that DTI is a sensitive method to detect white matter changes in subjects with $\mathrm{MCl}$ and $\mathrm{AD}$. It seems that in widespread brain regions diffusivity is already impaired early in the disease process, with more severe white matter disruptions with increasing disease severity. DTI abnormalities were found in many different regions. FA and MD changes seem to follow a slightly different anatomical pattern whereas highest ES (relative to controls) for DTI-FA were typically seen in cingulate and callosal areas (total cingulum, posterior cingulum, parahippocampal cingulum, splenium CC and uncinate fasciculus). For DTI-MD the largest differences were seen in the hippocampus, followed by the parietal lobe, splenium CC and temporal lobe. It was furthermore found that the discriminative power of DTI-MD is slightly higher than for DTI-FA.

Chapter 6 aimed to validate the findings of chapter 5 by means of a case control study. Cross-sectionally, we found a constrained pattern of GM differences in left parahippocampal gyrus (healthy controls versus subjects with $\mathrm{MCl}$ and $A D$ ) and left posterior cingulate regions (controls versus $A D$ ). This in contrast with previous findings that show a widespread pattern of GM atrophy in $A D$ and a more restricted $\mathrm{GM}$ atrophy pattern in $\mathrm{MCl}$ patients. In addition, diffusivity metrics were more sensitive for group differences than atrophy measurements, and were less influenced by the effects of aging, gender and scan parameters in a priori selected ROIs. Diffusivity differences were found in all the investigated structures, whereas changes in absolute diffusivity (mean diffusivity (MD), radial diffusivity (DR) and axial diffusivity (DA)) were more pronounced than changes in anisotropy (FA). We furthermore found that diffusivity differences in the genu of the corpus callosum but not volumetric measures predicted decline in a group of $\mathrm{MCl}$-subjects. Due to the time-intensive nature of manual volumetry, automated segmentation techniques become more and more popular nowadays. As a result, the question arises if processing conditions such as workstation type, operating system and software version affect segmentation results and thus influence group differences. In chapter 7 we investigated the robustness of FreeSurfer to capture morphological changes in the brain against varying processing variables and in comparison to manual 
measurements. We concluded that later versions of FreeSurfer were more accurate than earlier versions, especially in medial temporal and posterior parietal regions. A lot of research in the last decade has pointed out resting state functional connectivity as a marker for AD. The first step toward a biomarker is assuring that age-effects do not influence the reliability of RSNs.

In chapter 8 we investigated whether functional connectivity patterns can be reliably reproduced over time in healthy old and young subjects. It was found that measurements of functional connectivity, investigated by means of resting state functional MRI were stable over different time points, showing good to excellent reproducibility, both in healthy old and healthy young subjects. Age-effects were furthermore identified, indicating a decrease in functional connectivity in the DMN and in the auditory system, associated with aging.

Chapter 9 provides a general overview of the findings described in this thesis. Clinical and research implications resulting from the presented studies are discussed.

In conclusion, this thesis expands insights into the added value of imaging markers as diagnostic tool for the early detection of AD. Atrophy of MTL structures is a candidate marker to assess change from $\mathrm{MCl}$ to moderate $A D$ stages (prognosis). We showed that relatively novel and thus less validated MRI markers such as DTI and resting state functional MRI are promising for diagnosing $A D$ in an early stage but need to be evaluated empirically. Standardization of operating procedures (for e.g. volumetric and connectivity approaches) will further consolidate our understanding of AD-pathology, facilitate comparison and integration of results, enable the establishment of cut-offs and consequently pave the way to clinical implementation. 




\section{NEDERLANDSE SAMENVATTING}

De ziekte van Alzheimer (ZvA) is de meest voorkomende vorm van dementie die wereldwijd ongeveer 34 miljoen mensen treft. Onderzoekers voorspellen een tsunami van dementie, waarbij de prevalentie van de ziekte zal verdriedubbelen als gevolg van de vergrijzing van de bevolking. Dit leidt tot een toenemende nood aan vroegdiagnostiek van deze ziekte om behandeling in een vroege fase mogelijk te kunnen maken. Een vroege diagnose en behandeling kan immers naast een toename van levenskwaliteit ook een kostenbesparing teweegbrengen, zowel op het niveau van de patiënt als van de gezondheidszorg. Vooraleer klinische of cognitieve afwijkingen daadwerkelijk objectiveerbaar worden, is er reeds sprake van onderliggende abnormaliteiten op hersenniveau, waarbij volumeverlies in bepaalde regio's en een afname van cerebrale connectiviteit een voorbeeld zijn. Beeldvorming met magnetische resonantie (NMR of MRI) biedt een kader om de structurele en functionele veranderingen geassocieerd met het proces van neurodegeneratie te visualiseren.

Het onderzoek beschreven in dit proefschrift kadert binnen het eerder aangehaalde onderzoeksveld van vroegdiagnostiek en heeft primair als doel de inzichten in het gebruik van zowel de traditionele (bijv. volumebepaling van de hippocampus) als de meer recente (bijv. diffusiviteit in de witte stof) beeldvormingstechnieken te vergroten. Deze technieken werden geëvalueerd zowel op het vlak van vroegdetectie, diagnose, als prognose van de ziekte van Alzheimer. De huidige aanpak in de meeste Nederlandse geheugenpoli's vertrekt van een interview met patiënt en zijn naaste, gevolgd door een neurologisch onderzoek. Hierna wordt een neuropsychologische test batterij afgenomen en vindt er een bloedtest en een hersenscan plaats. Aangezien MRI beelden ons meer informatie kunnen verschaffen dan zuiver macroscopische (bv. tumor) of vasculaire afwijkingen, is het gebruik van hersenscans in de kliniek van exclusie van andere ziekten (bv. tumoren, infarcten) uitgebreid naar vroegdetectie (bv. ZvA versus gezonde veroudering) en differentiaaldiagnostiek (bv. ZvA versus Fronto-temporale dementie). Echter, de toegevoegde waarde en exacte rol van de nieuwe beeldvormingstechnieken voor de vroegdiagnostiek van de ZvA is nog steeds onduidelijk. De vraag of MRI onderzoek überhaupt noodzakelijk is in het diagnostisch proces van de ZvA en welke MRI technieken potentieel zinvol zijn vormde het startpunt van dit proefschrift. 
De klinische waarde van nieuwe beeldvormingstechnieken voor de ZvA werd geëvalueerd in relatie tot de meer traditionele ('gouden standaard') methoden (bv. volumemeting van de hippocampus en een visuele beoordelingsschaal voor volumeverlies van de mediale temporaalkwab).

In het eerste deel van dit proefschrift (Hoofdstuk 2-4) werden technieken die volumeverlies in de grijze stof van het brein meten geëvalueerd. Het tweede deel (Hoofdstuk 5-6) beschrijft het onderscheidend en voorspellend vermogen van een nieuwe beeldvormingstechniek: Diffusie-gewogen beeldvorming ('diffusion tensor imaging' of DTI). Het derde en laatste deel van dit proefschrift (Hoofdstuk 7-8) heeft zich gericht op het evalueren van de robuustheid van structurele en functionele afwijkingen bij de ZvA.

In hoofdstuk 2 hebben we in een multicenter setting onderzocht $(n=184)$ of het toevoegen van MRI-beelden de voorspellende waarde voor de ZvA verhoogd ten opzichte van een model dat enkel demografische en neuropsychologische informatie bevat. Hippocampaal volume (gemeten op MRI-beelden) bleek de beste voorspeller te zijn voor conversie van milde cognitieve klachten $(\mathrm{MCl})$ naar de ZvA. Het toevoegen van het volume van de hippocampus verbeterde bovendien de voorspellende waarde van een episodische geheugentaak. In lijn met eerder onderzoek werd gevonden dat een test voor uitgestelde reproductie van een woordenlijst de beste voorspeller is voor de ZvA op neuropsychologisch vlak. We concludeerden dat wanneer MRI-onderzoek mogelijk is (oa. infrastructuur en expertise aanwezig, patiënt MRI-compatibel), dat een volumemeting van de hippocampus naast neuropsychologisch onderzoek wenselijk is. Verder bleek dat de toegevoegde waarde van een visuele beoordelingsschaal (MTA-score) beperkt was in termen van sensitiviteit en specificiteit.

Het evalueren van volumeverlies in de mediale temporaalkwab is tot op heden nog steeds een belangrijk onderdeel van de diagnostiek bij de ZvA. Volumeverlies in deze regio kan bovendien converters van non-converters onderscheiden en mensen die lijden aan de ZvA onderscheiden van mensen met een ander type dementie. In hoofdstuk 3 hebben we de voorspellende waarde van 4 verschillende maten die volumeverlies van de mediale temporaalkwab meten onderzocht in een multicenter $(n=328)$ en single center $(n=172)$ setting. Resultaten hebben aangetoond dat een volumemeting van de hippocampus de beste voorspeller is voor conversie naar 
de ZvA bij mensen met $\mathrm{MCl}$ (zowel de manuele als automatische meting van hippocampaal volume (LEAP methode)). Beide maten toonden bovendien een sterke samenhang met markers in hersenvocht (CSF) die schade aan de hersencellen meten (CSF t-tau en p-tau). Er werden consistente afkapwaarden gevonden tussen verschillende cohorten en beide maten voorspelden cognitieve achteruitgang na 2 jaar. De LEAP volume meting heeft als voordeel ten opzichte van de manuele methode dat deze minder tijdsintensief is en geen variabiliteit tussen beoordelaars laat zien. Bovendien heeft LEAP een erg lage technische foutenmarge.

Recent onderzoek heeft aangetoond dat volumeverlies in de mediale temporaalkwab niet geheel specifiek is voor de ZvA en dat we dit ook terugvinden bij andere vormen van dementie. Uit eerder onderzoek is bovendien gebleken dat Alzheimer pathologie zich niet beperkt tot deze gebieden. Aangezien ook andere regio's aangetast zijn bij de ZvA hebben we in hoofdstuk 4 onderzocht welke de onderscheidende waarde is van verschillende technieken die volumeverlies in de grijze stof van de hersenen meten, zowel in de mediale temporale gebieden als daarbuiten. We hebben onderzocht of 4 verschillende technieken die grijze stof atrofie in het brein meten dezelfde resultaten laten zien binnen eenzelfde dataset. De sensitiviteit of gevoeligheid van elke methode bleek afhankelijk van de onderzochte regio. Resultaten toonden aan dat een manuele volumemeting en VBM (voxel based morphometry) de voorkeur genieten voor het in kaart brengen van volumeverlies in de grijze stof. De meeste regio's lieten groepsverschillen zien met een duidelijk overwicht in mediaal temporale gebieden. Automatische volumetrische methodes (FreeSurfer) toonden gelijkaardige prestaties waarbij verschillen in de posterieure delen van de parietaalkwab (en dus niet in de mediaal temporaalkwab) het meest uitgesproken waren in de vroege fase van de ziekte. Het meten van de dikte van de hersenschors ('cortical thickness') bleek minder gevoelig in het vroege stadium van de ziekte.

In hoofdstuk 5 hebben we een meta-analyse gepresenteerd die primair als doel had de diagnostische waarde van een nieuwe beeldvormingstechniek (DTI of diffusie tensor beeldvorming) te evalueren in een groep van gezonde controles, patiënten met $\mathrm{MCl}$ en patiënten met de ZvA. Naast 76 studies die volumeverlies in de mediale temporaalkwab onderzochten (8122 subjecten) werden er ook 55 DTI studies (2791 subjecten) geïncludeerd. Voor verschillende regio's in het brein werden ef- 
fectgroottes berekend die op hun beurt vergeleken werden met effectgroottes van technieken die atrofie van de mediale temporaalkwab meten. Resultaten toonden aan dat in wijdverspreide regio's van het brein de beweging van watermoleculen (diffusie) reeds in een vroege fase van de ziekte verstoord is, waarbij we meer ingrijpende witte stof veranderingen zien bij progressie van de ziekte. Abnormaliteiten op het vlak van fractionele anisotropie en gemiddelde diffusiviteit toonden een verschillend anatomisch patroon. De maat van anisotropie was het meest gevoelig voor verschillen in cingulate en callosale regio's. De gemiddelde diffusiviteit daarentegen was het meest sensitief voor verschillen in de hippocampus, gevolgd door de parietaalkwab, het splenium van het corpus callosum en de temporaalkwab. Over het algemeen genomen was de fractionele anisotropie index even gevoelig als de gemiddelde diffusiviteit. Een lagere gevoeligheid van fractionele anisotropie ten opzichte van gemiddelde diffusiviteit was het meest opmerkelijk in de corticale gebieden.

In hoofdstuk 6 hebben we gepoogd de bevindingen van hoofdstuk 5 te valideren door middel van een case control studie. Onze resultaten toonden aan dat het patroon van grijze stof afwijkingen in deze studie beperkt was tot de linker parahippocampale gyrus (controles versus subjecten met $\mathrm{MCl}$ en ZvA) en de linker posterior cingulate (controles versus subjecten met ZvA). Dit is deels in tegenstelling met eerdere bevindingen die een wijdverspreid patroon van afwijkingen laten zien bij patiënten met de ZvA en een meer beperkt patroon van grijze stof afwijkingen bij MCl-patiënten. Er werd daarenboven gevonden dat diffusiviteitsmaten meer gevoelig zijn voor groepsverschillen dan metingen van volumeverlies en dat deze minder beïnvloed worden door leeftijdseffecten, effecten van geslacht en scanner instellingen. Verschillen qua diffusiviteit werden gevonden in alle onderzochte structuren waarbij veranderingen in absolute diffusiviteit (gemiddelde diffusiviteit $(M D)$, radiale diffusiviteit (DR) en axiale diffusiviteit (DA)) meer uitgesproken waren dan veranderingen in anisotropie (FA). Verder werd gevonden dat niet het volumeverlies maar de verschillen in diffusiviteit in de genu van het corpus callosum achteruitgang voorspelden in een groep van $\mathrm{MCl}$-patiënten. Door het tijdsintensieve karakter van manuele volumemetingen hebben automatische metingen meer en meer aan belang gewonnen. De potentiële invloed van hardware en software instellingen op de resultaten van dergelijke technieken en dus ook het indirecte effect op groepsverschillen is tot nog toe vrij onderbelicht gebleven. In hoofdstuk 7 onderzochten we of FreeSurfer resistent is tegen deze verschillen. Om hierbij een 
zuivere vergelijkingsbasis te hebben werd deze techniek rechtstreeks vergeleken met de manuele segmentatie. Onze bevindingen toonden aan dat het effect van hardware (workstation) kleiner is dan van software (FreeSurfer versie) en dat latere versies van FreeSurfer meer accuraat zijn dan eerdere versies, vooral in posterieur pariëtale gebieden.

Recent onderzoek heeft aangetoond dat 'resting state' functionele MRI (rsfMRI), een techniek die de hersenactiviteit in een taakvrije omgeving meet, een belangrijk marker is voor de ZvA. De eerste stap richting ontwikkeling en validatie van een zogeheten 'biomarker' is onderzoeken of leefijdseffecten de betrouwbaarheid van deze 'resting state' netwerken niet beïnvloeden. In hoofdstuk 8 werd onderzocht of patronen van functionele connectiviteit stabiel zijn over de tijd bij gezonde ouderen en jongeren. Resultaten toonden aan dat metingen van functionele connectiviteit, onderzocht door middel van rsfMRI, stabiel waren over verschillende tijdsmomenten, en een goede tot uitstekende reproduceerbaarheid laten zien. Leeftijdseffecten werden geïdentificeerd en er werd een afname in functionele connectiviteit gevonden in het 'default mode netwerk' (DMN) en het auditieve systeem, welke in verband staan met veroudering.

In hoofdstuk 9 wordt een algemene discussie van onze bevindingen beschreven. Klinische en wetenschappelijke implicaties worden hier uitgebreid toegelicht en suggesties voor verder onderzoek worden gegeven.

We kunnen besluiten dat volumeverlies in de mediale temporaalkwab een kandidaatmarker is voor het meten van progressie van een milde cognitieve stoornis naar de ZvA (prognostische waarde). Het onderzoek hier beschreven toont bovendien aan dat relatief nieuwe en dus minder gevalideerde MRI technieken (DTI en resting state $\mathrm{fMRI}$ ) veelbelovend zijn voor de toekomstige vroegdiagnostiek maar dat deze nog uitgebreid empirisch getest en gevalideerd moeten worden. Standaardisatie van de procedures (voor bijv. volumetrische maten en maten van structurele en functionele connectiviteit) zullen onze kennis wat betreft onderliggende Alzheimer pathologie verder uitbreiden en ervoor zorgen dat we resultaten kunnen vergelijken en integreren. Bovendien kunnen we op die manier stabiele afkappunten gaan ontwikkelen dewelke de weg naar klinische implementatie verder kunnen faciliteren. Het ultieme einddoel van een MRI-biomarker is immers een betrouwbare diagnose op subjectniveau, gebaseerd op een enkele MRI-scan. 



\section{CURRICULUM VITAE}

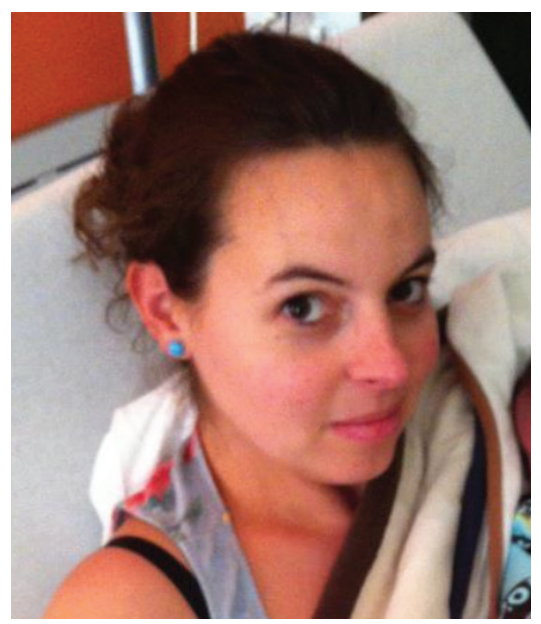

Lies Clerx was born on October, $25^{\text {th }} 1982$ in Diest, Belgium. After completing secondary school at the Voorzienigheid Diest, she obtained a bachelor degree in Information Technology at the PHL in Hasselt. In 2004 she started studying Clinical Psychology at the University of Leuven. As a result of her growing interest in neuropsychology, the subject of her master thesis at the lab of Biological Psychology in Leuven was the impact of hippocampal lesions on different

forms of spatial and non-spatial learning (supervision: Prof. Dr. R. D’Hooge) (Summa Cum Laude). During her master she also completed a clinical internship at the Psychiatric Hospital/ University Center in Kortenberg (Leuven) were she gained a lot of diagnostic experience with patients suffering from Alzheimer's disease and other dementias. This again stimulated her interest in ageing and neurodegenerative diseases. After obtaining her master's degree in Psychology (Cum Laude) in June 2009, she started as a PhD student at the Department of Psychiatry and Neuropsychology at Maastricht University in August 2009. She investigated the value of novel markers for the early diagnosis of Alzheimer's disease under the supervision of Prof. Dr. F. Verhey, Dr. P. Aalten and Dr. P.J. Visser. During her PhD, Lies' work was rewarded with a poster prize on the Euron PhD days and several presentations at international conferences. From June to September 2012 she worked as an intern at the Forschungszentrum in Juelich, Germany on a research project which focused on task-free brain activity in healthy aging. During her PhD trajectory, Lies also enrolled in a 2-year Master Specialization 'Clinical Neuropsychology' at the Free University of Brussels of which she graduated in July 2013 (Summa Cum Laude). Assuming that there was no life after obtaining your PhD degree, she grip her Phd-time to build a house, to give birth twice and to marry the man of her life. In January 2014, Lies started as a post-doctoral fellow at the Forschungszentrum in Juelich, Germany. 



\section{PUBLICATIONS}

\section{International Publications}

* Clerx L., Visser P.J., Verhey F.R.J., Aalten P. (2012). New MRI markers for Alzheimer's disease: a meta-analysis of diffusion tensor imaging and a comparison with medial temporal lobe measurements, Journal of Alzheimer's Disease, 29(2), 405-29.

Clerx L., van Rossum I.A., Burns L., Knol D.L., Scheltens P., Verhey F.R.J., Aalten P., Lapuerta P., van de Pol L., van Schijndel R., de Jong R., Barkhof F., Wolz R., Rueckert D., Bocchetta M., Tsolaki M., Nobili F., Wahlund L.O., Minthon L., Frölich L., Hampel H., Soininen H., Visser P.J. (2013). Measurements of medial temporal lobe atrophy for prediction of Alzheimer's Disease in subjects with mild cognitive impairment, Neurobiology of Aging, 34(8),

- Clerx L., Jacobs H.I., Burgmans S., Gronenschild E.H., Uylings H.B., Echávarri C., Visser P.J., Verhey F.R.J., Aalten P. (2013). Sensitivity of different MRItechniques to assess gray matter atrophy patterns in Alzheimer's Disease is Region-Specific, Current Alzheimer Research, 10(9), 940-51.

Jacobs H.I., Clerx L., Gronenschild E.H., Aalten P., Verhey F.R.J. (2014). White Matter Hyperintensities are Positively Associated with Cortical Thickness in Alzheimer's Disease, Journal of Alzheimer's Disease, 39, 409-422.

* Clerx L., Dierckx E., van de Pol L., van Rossum I., Verhey F., Aalten P., Barkhof F., Wolz R., Rueckert D., Frisoni G., Tsolaki M., Nobili F., Freund-Levi Y., Wallin A., Frölich L., Hampel H., Soininen H., Scheltens P., Visser P.J. Added value of MRI-markers to neuropsychological test performance for the prediction of $A D$ in subjects with $\mathrm{MCl}$. Submitted.

* Clerx L., Gronenschild E., Echávarri C., Verhey F., Aalten P., Jacobs H. Can FreeSurfer compete with manual volumetric measurements in 

Alzheimer's disease? Submitted.

Clerx L., Jacobs H., Gronenschild E., Backes W., Olde Rikkert M., Claassen J., Barkhof F., Binnewijzend M., van Buchem M., van der Grond J., Visser P.J., Verhey F., Aalten P. Reduced callosal white matter integrity surpasses cerebrospinal fluid and atrophy markers as a predictor of decline in subjects with $\mathrm{MCl}$ : a combined volumetry and DTI study. Submitted

Clerx L., Kukolja J., Göreci Y., Hafkemeijer A., Onur O., Aalten P., Verhey F., Fink G., Jacobs $H$. The stability of resting state networks in aging: relevance for early memory dysfunction. Submitted.

Jacobs H.I., Dillen K., Clerx L., Goreci Y., Onur O.A., Fink G., Kukolja J. Consolidation in older adults depends upon competition between taskfree networks. Submitted.

\section{National publications}

* Lies Clerx, Pauline Aalten. (2012). De meerwaarde van diffusie gewogen beeldvorming bij de vroegdiagnostiek van de ziekte van Alzheimer, Neuropraxis, 2012, 5(5), 143-148. 



\section{DANKWOORD}

Beste (ex)collega, vriend, familie, verdwaalde lezer,

Meer dan vier jaar geleden, op een regenachtige lentedag (uiteraard in België) kreeg ik onderweg van Leuven naar huis telefoon. Een nummer uit NL... Zou het? Ik durfde amper te antwoorden: "Hallo Lies, Pauline Aalten uit Maastricht, stoor ik?" "Euh nee hoor" *slik* "Wij hebben besloten dat jij de ideale kandidaat bent voor de MRI onderzoeksplek binnen het LeARN project..." *say whaaaaat* "...dus als jij het ook ziet zitten verwelkomen we jou graag binnenkort in Maastricht". Hoe graag ik ook "Jaaaaa natuuuurrrllliiijjk" wou schreeuwen... toch probeerde ik de rustige Lies te zijn (bestaat-die-dan?) en antwoordde ik "Ja hoor, heel graag" ...

Het enthousiasme wat er was op dat moment en de zin om 'erin te vliegen' is sedert dien alleen maar gegroeid. Nooit gedacht dat ik op 4 jaar tijd zoveel zou ontdekken, verwezenlijken, bijleren. Dit promotietraject was voor mij een verrijking op alle vlakken.

4 jaar en minstens evenveel life-events later kijk ik terug op een heerlijke tijd. Een tocht naar de bouw van dit proefschrift die mede mogelijk werd gemaakt door een aantal mensen die er elk op hun manier voor gezorgd hebben dat ik -in de meest optimale omstandigheden- deze promotie tot een goed einde kon brengen. Ik neem dan ook graag de tijd om iedereen uitgebreid te bedanken, "voor die ene keer dat het eens kan en mag gezegd worden, en dat er geen beperking op het aantal woorden zit".

Allereerst wil ik graag alle patiënten, hun mantelzorgers en de gezonde proefpersonen bedanken. Zonder jullie was dit onderzoek niet mogelijk geweest en dankzij jullie deelname en het beschikbaar stellen van de klinische gegevens staan wij onderzoekers elke dag een stapje dichter bij het oplossen van het Alzheimervraagstuk. Bedankt voor uw tijd en vertrouwen.

\section{Mijn promotieteam:}

Frans, het feit dat je promotor bent van minstens evenveel promovendi als dat er dagen in een maand zijn (parallel gezien dan) belette je niet om telkens actief 
mee te denken over nieuwe projecten en om me er steeds aan te herinneren wat het uiteindelijke doel van dit ganse traject was. Je probeerde me telkens op het juiste pad te houden wanneer ik er vanaf dreigde te dwalen. Bedankt voor het vertrouwen en de autonomie om zelf richting te mogen geven aan mijn verschillende onderzoekslijnen! En dank je wel om steeds de tijd de nemen om te peilen 'of het met de kindjes thuis wel allemaal goed te combineren viel'.

Pieter-Jelle: van jou leerde ik dat er voor alles een syntax bestaat, dat alles te verklaren valt, dat iemand zowel K3 als klassieke muziek kan waarderen en dat iemand tegelijk verstrooid en perfectionistisch kan zijn. Deze laatste combinatie leidt blijkbaar zelfs tot onderzoek op topniveau! Bedankt dat ik mee mocht genieten van jouw kennis en kunde, en bedankt om steeds voor mij de tijd te nemen ondanks jouw ontelbare andere taken/verantwoordelijkheden/AIO's.

Pauline, bij de start van mijn promotie slaagde jij er meteen in mij op mijn gemak te doen voelen en daarnaast toch ook de nodige professionaliteit uit te stralen om een goede dagelijkse begeleider te kunnen zijn. Fijn trouwens om grote zusjes en kleine broertjes ervaringen te kunnen uitwisselen! Bedankt dat jouw deur steeds open stond voor werk en wat-minder-werkgerelateerde vragen en bedenkingen.

Heidi, mijn niet-officiële maar daarom niet minder waardevolle co-promotor: waar moet ik beginnen? Ik ga voor Heidelberg! Van toen af hebben wij het heel erg met elkaar kunnen vinden, op alle vlakken. Heerlijk hoe jij altijd weer energie en zin hebt om mee te denken over allerlei dingen. Ik heb het gevoel dat ik met jou volledig op 1 lijn zit. Mede dankzij jou heb ik een ongelofelijk leuke tijd gehad tijdens mijn stage in Jülich en is mijn kennis op het vlak van MRI op tijd en stond serieus uitgebreid (nerdtime that is!). Ik heb enorm veel bewondering voor alles wat je verwezenlijkt hebt en datgene waar je nog elke dag naar streeft (en liefst zoveel mogelijk tegelijkertijd). Ik wil je graag bedanken voor alles. Graag samen ook nog veel california maki's met een cola light/zero (en smashed apples;-)) en ik zorg ervoor dat ML en M nog veel tekeningen voor jou (en Joost) zullen maken ;-) Bedankt om er altijd te zijn en om altijd tijd voor me te willen maken! 
I would gratefully like to thank the members of my reading committee for their time and involvement in evaluating my work: Prof. Dr. Yasin Temel, Prof. Dr. Stefan Teipel, Prof. Dr. Mathieu Vandenbulcke, Dr. Paul Hofman and Dr. Inez Ramakers.

\section{Mijn MRI-buddies:}

Saar, ik bewonder jouw vastberadenheid en doorzettingsvermogen en wens je nog veel succes in je verdere onderzoekscarriere! Verder hoop ik ook dat we -als mama's- nog veel kunnen kletsen over onze kleine supermannen-en vrouw(en)!

Ed-gouden-raket, soms tovenaar altijd expert! Is er iets wat jij niet kan? Ja! ik weet het: water drinken! :) Bedankt voor alle hulp, door jou leek niks onmogelijk. Misschien moeten wij samen de DTI-bijbel schrijven? Daar kan je straks vast de tijd voor vinden! Of toch niet?! :) Ik wens je het allerbeste toe samen met je geweldige vrouwtje en ik hoop dat ik nog heel vaak centimeters lange emails van jouw mag ontvangen! (en niet met blessures of issues aub!).

Harm, ookal zitten er wat (kilo?)meters tussen onze werkplekken toch kreeg ik het gevoel dat wij altijd erg efficiënt en snel konden communiceren. Nog veel succes met je verdere promotie.

Mijn 'oude' kamergenootjes:

Ron, ik heb nooit iemand van onze leeftijd zo serieus en gewetensvol geweten als jij. En toch was er bij jou ook steeds plaats voor grapjes en grollen als een NederlandsVlaams woordenboek - waar is dat trouwens gebleven? Jij slaagt er bovendien in voor alles een lijstje te maken en alles te automatiseren. Petje af! Bedankt voor de interessante discussies en jouw heldere kijk op het hele LeARN gebeuren. Ik ben benieuwd hoe Papa-Ron zich verder zal ontplooien, graag af en toe een (pamper) update! :)

Syenna, jij prettig gestoorde kip tandoori. Jij bent zo iemand waarmee je na 5 min het gevoel krijgt je al een gans leven te kennen. Ik wil je nog veel succes de komende tijd! En als je graag nog eens een make-over van je huis hebt, gewoon even bellen en ik kom langs met de (b)engeltjes! ;)

Steph, lieve Steph, wat hebben wij een leuke tijd gehad samen daar aan die dubbele 
bureau in de glazen kamer! Geen koekje of thee-tje wat we op die jaren niet geproefd hebben en geen doctoraatsdipje wat we niet met elkaar hebben gedeeld. Zelfs een transfer naar de Albert Heijn zou destijds geen straf geweest zijn als ik met jou mocht samenwerken. Blij dat jij er telkens was tijdens de ontdekkingstocht van onze promotie en blij dat jij mijn paranimf bent op deze 'grote dag'... Ik hoop dat we in de toekomst nog vaak tegenover elkaar zullen zitten!

Renske, jij kwam er later bij (zoals zovelen) maar toch slaagde jij er vrijwel meteen in om actief mee te varen in het 'LeARN clubschuitje'. Fijn dat onze mannen het ook zo goed met elkaar kunnen vinden zodat onze contacten zich niet enkel beperken tot Maastrichtse bodem. Waar gaan we de volgende keer dineren en degusteren? Leuk dat jij eveneens dicht bij me bent tijdens mijn verdediging!

Gisela, mama Gis.. onze kamers lagen de afgelopen jaren 'ver' uit elkaar maar toch vonden we ieder om beurt de tijd om lekker even bij te kletsen (en voor nu: leve whatsapp!). Fijn om samen met jou dikke buik en andere kindergerelateerde pret te kunnen delen.

Sanne, Peggy en leke, mijn ex-buurvrouwen: leuk dat jullie de witte en andere chocotoffs met mij wilden delen en tegelijk ook steeds into een leuke babbel waren! leke: jouw gouden tip van M\&Ms eten in de auto en dan het pakje naar achter gooien - ik pas hem regelmatig toe! Dank je wel daarvoor ;) S\&P: veel succes nog met het afronden van jullie boekje!

Nico en Ron M., wat mij betreft zijn jullie de levende versie van de Rescue Rangers: geen karwei te groot, geen klus te zwaar, die 2 staan altijd klaar! De verdeling van wie knabbel en wie babbel is laat ik aan de lezer over. Bedankt voor jullie IT-hulpin-nood!

Elsa, "la mama" van de afdeling! Voor alles kunnen we bij jou terecht - en we worden bovendien nog ontvangen met een brede glimlach ook. Bedankt voor alle hulp tijdens mijn promotie en voor het extra-tandje-erbij bij de afronding!

Astrid, bedankt voor jouw logistieke en andere hulp bij het organiseren van NPO's, huisbezoeken, scansessies enz.! 
Tanja en Carla: bedankt voor jullie ondersteuning en harde werk tijdens de vele patiëntencontacten!

Claire, bedankt voor de leuke samenwerking binnen PSI/LeARN!

Marjolein H., we hebben elkaar niet zo vaak 'in person' gezien de afgelopen jaren maar we hebben samen wel een erg leuke tijd gehad in Riga (oa. in die Amerikaanse eettent haha) en gelukkig bestaat er social media die veel mogelijk maakt! Veel succes met de opleiding verder!

Alle andere collega's van divisie 1 wil ik eveneens bedanken voor de leuke tijd samen!

I would also like to thank my German colleagues: Eckart, we had some nice low-level conversations im Seecasino, thanks for your daily help in choosing the right sausage for lunch. Kim and Niels (and later: Boris), we had a great time together in the summer of 2012! I enjoyed our FSL-discussions and the little course we organized. Ozgur, our contact is limited but however valuable. It's nice to have little boys who share the same age. Juraj, thank you for giving me the opportunity to work on the RIMCAD data.

Uiteraard en niet in het minst zijn er ook een heleboel mensen buiten Maastricht die het verdienen om hier vernoemd te worden:

Lieve Anneke, Moorsie, my BFF! Zo leuk dat we ineens terug in elkaars leven zijn gekomen - it was meant to be! Heerlijk om samen ADA te zijn en uren met jou te messagen, bellen of chatten. YOLO! Elkaar in het echt zien is natuurlijk nog veel beter maar dan lukt het ongeneerd praten niet zo goed door die 4 kleine varkentjes van ons. Ik hoop dat ik nog vaak spreuken mag sturen omdat je overtijd gaat.. en dat jij nog vaak (kuch) als eerste op de materniteit kan staan. Voor de geur alleen al, toch? :) (ps: wa IS dees?!).

Lieve Ellen S. aka foefie, we kennen elkaar nu zo'n 13 jaar (say whaaaat?) en ik herinner me nog heel goed wat een leuke tijd we hebben gehad op de PHL. Fully open, samen met Mie. Ik ben blij dat we nog steeds contact hebben en dat we 
elkaar bij leuke en minder leuke dingen altijd weten te vinden. 2014 kan niet meer stuk, jullie wens mag eindelijk uitkomen... en die van Fre en mijzelf dus ook een beetje! Muchos besos voor jou en Steve-man!

Lieve Ellebelle, hoe krijgen we het toch voor elkaar om telkens weer dezelfde keuzes te maken in het leven? Zo fijn om een blond kopietje van mezelf te hebben! Tha blond and tha brunette! Foreva! Ik kan heel wat wist-je-datjes gaan bedenken maar eentje blijft essentieel: groene fluostift + tranen = gele fluostift (ik WIST van bij het ontstaan van die spreuk dat deze ooit gepubliceerd ging worden: bij deze!) ;) Keep on shining Miss van Criekinge! En jij ook nog veel succes met afrondingsmanoeuvres - ik zal er zijn he! ;)

Lieve Stephanie T., Celine, Ann. R., Nathalie, Sylvie, Katrien V., Vroni - bedankt voor jullie oprechte interesse in mijn doen en laten van de afgelopen 4 jaar. Een mens kan zich geen betere vriendinnen wensen!

Graag wil ik ook mijn bredere familie en schoonfamilie bedanken voor hun interesse in de ongetwijfeld vaak abstracte inhoud van mijn job. Fijn dat jullie vanuit de kantlijn zo enthousiast wilden mee supporteren!

Steven, dank je wel voor het prachtige ontwerp van de kaft! Een half uurtje hebben we hiervoor samen gezeten - ik probeerde mijn vage ideeën zo goed mogelijk over te brengen en jij noteerde HELEMAAL NIETS. Ik wachtte rustig (en toch ook wat sceptisch) af tot ik enkele weken later een knaller van een cover toegestuurd kreeg - ongelofelijk! PAL EROP! Exact wat ik wou en dat zonder enige voorkennis en met een schamele uitleg - dat kan alleen een echte professional.

Kristof, lieve schoonbroer (of is het schoonboer???), fijn dat we op tijd en stond eens nuchter (?) kunnen kletsen over het reilen en zeilen binnen onze beide jobs. Hoewel erg uiteenlopend weten we allebei wat het is om gebeten te zijn door ons vak, hard te werken en een heel klein beetje veel work-a-holic te zijn. Ik vind het heerlijk om zo een leuke schoonboer te hebben! Bedankt om bovendien zo'n leuke peter/nonkel te zijn voor onze kids!

Nico, je suis heureuse que tu fasses partie de la famille. Tu es l'homme grâce à qui 318 | Appendix 
ma petite soeur n'a pas mal tourné! :) Et n'oublie jamais: kleine $t^{* * * *}$ and dikke $\mathrm{p} * * * !:)$

Elisabeth, lieve schoonzus, wat heerlijk dat jij er plots bijkwam. Eindelijk iemand waarmee ik ongegeneerd kon kletsen, shoppen en chocola eten. Een lieve tante Bee voor ML, een uitmuntende metie voor Maurice en een geweldige vriendin voor mij. Ik hoop dat we samen nog veel stukken chocola kunnen kraken, voor-, tijdens en na onze shoppinguitjes!

Sonia, lieve zus, we kennen elkaar ondertussen iets minder dan 30 jaar en hoewel we $400 \mathrm{~km}$ uit elkaar wonen, toch slagen we er telkens in om bij belangrijke gebeurtenissen bij elkaar op de 1e rij te staan. Mijn jeugd had er heel anders uit gezien zonder jou erbij, dank je voor alle leuke momenten en ook om ze allemaal te onthouden zodat we ze nu elke keer weer opnieuw kunnen beleven. A demain, portemonnaie vous bien.. En dan? :) Ma das de wiiinnnddd! :p ML kan zich geen betere meter wensen en ik geen betere zus. Sokke, you rock BIG time!

Marc en Monique: schoonouders krijg je er gratis bij - Wel, voor jullie had ik gerust heel wat willen bijbetalen! Ongelofelijk hoe goed het tussen ons allemaal klikt en wat voor een prettig gestoorde bende we samen zijn. Ik hoop dat we nog vaak samen kunnen 'zakdoek leggen' op Italiaanse (of andere) bodem. Marc, bedankt om ons telkens op culinair en ander vlak in de watten te leggen, geen zalm is veilig in jouw buurt! Monique, mémé, mijn respect voor jou is eindeloos. Ondanks het ziek zijn, de pijn en de moeilijke periodes sta je toch altijd klaar om ons te helpen als dat nodig is. $100 \mathrm{~km}$ rijden om mijn achterstallige berg strijk te komen doen geschiedt met de glimlach, ML en M opvangen, een luisterend oor bieden en een flinke dosis amusement voorzien; niets is teveel gevraagd. Mede dankzij jou (jullie) is dit doctoraat geworden wat het vandaag is en daar kan ik je niet genoeg voor bedanken!

Mijn rotsen in de branding: mama en papa! Eerst en vooral sorry voor het bloed, het zweet en de tranen die ik jullie gekost heb tijdens mijn kleuter-, tiener- en puber-tijd. Vechten om niet naar het oudercontact te moeten gaan, moedeloos thuiskomen en ontvangen worden door een kind dat blijft volhouden dat 'alles goed komt'. Maar hey, had ik gelijk of had ik gelijk? Duizend maal dank om me bij te sturen 
waar nodig, maar toch ook vrij te laten in de keuzes die ik wou maken en uiteraard om mij alle kansen te geven! Bedankt om er altijd te zijn voor ML en M, om bij te springen waar nodig en om urenlang mijn klaagzangen te aanhoren (vooral tijdens mijn Leuven-tijd: 'Ik ga effe wenen he, maar eet gerust verder ge moet ni naar mij kijken'). Papa, bedankt voor je bescheiden maar steeds übernuchtere kijk. Ik ben er zeker van dat er niemand is die met nog minder woorden zoveel duidelijk kan maken. Het is er gewoon altijd 'boenk op'! Ookal zijn het vaak niet de zaken die ik op dat moment wil horen. Ik heb enorm veel bewondering voor alles wat je bereikt hebt in je leven en ook voor alles waar je tot op de dag van vandaag nog voor gaat. Hoezo maar ene keer per verlof de Ventoux doen?! Mijn superpapa! Mama, er zijn maar weinig dochters die het geluk hebben dat hun moeder EN een moeder EN een beste vriendin is. Er is niets waarmee ik bij jou niet terecht kan. Wellicht kunnen we vaak met veel minder woorden iets aan elkaar duidelijk maken maar hey wat hindert dat, happytime nemen wij gewoon heel letterlijk. Ik kan nog pagina's vullen maar het boekje is uiteindelijk al dik genoeg dus het volstaat om hier af te ronden met een welgemeende dankjulliewel en een 'ikziejullieongelofelijk graag'!

Fre, schattie, hubbie, ondertussen zijn wij al meer dan 6 jaar samen en ik kan me niet herinneren dat ik mij ooit 1 seconde verveeld heb. Sinds april 2008 staat de funmode in mijn leven permanent aan. Het dagelijks leven met jou en de kids is gewoon DE MAX! Ik hoop dat wij, 2 prettig gestoorde psychologen, nog veel crazy avonturen samen kunnen beleven. Soms (heel soms dan wel) zijn woorden overbodig: Ik hou zielsveel van je!

En dan nu de aller aller aller allerbelangrijkste persoontjes in mijn leven: DE KIDS!

Lieve Marie-Lou en Maurice,

Zonder jullie ... was het zéééér zéker OOK gelukt... haha! Grapje!

Marie-Lou, Louke, Louisie, pippi langkous, mijn mooie lieve prinses: jij bezorgt papa en mij al meer dan 3 jaar topentertainment van de bovenste plank. Mijn liefde voor jou en broer is grenzeloos. Ik hoop dat we nog vaak samen kunnen shaken op K3 en dat ik nog vaak voor jou slaapwel verhaaltjes mag verzinnen. Lieve meid, ik kijk uit naar alles wat nog komt en ben superfier elke dag opnieuw jouw mama te mogen zijn. Love you van hier tot aan de maan en terug! 
Maurice, Mr. Mau, Maurizio, broeder Tuc, stoere man van me: vanaf de eerste minuut was ik totaal aan jou verhangen. Jouw grote donkere deugenieten-kijkers doen me elke dag opnieuw beseffen wat écht belangrijk is. Ik hoop dat jij en zus nog vaak apenkuren gaan uithalen en ons huis overhoop gaan zetten - gooi die dvd's maar allemaal door de kamer, haal die kasten maar leeg! Ik kijk er naar uit om ook dan weer samen met papa de uitdaging aan te gaan in het heerlijke opvoedingsavontuur. Want sinds jij in onze leven bent is die uitdaging wel minimaal verdriedubbeld!!! Love you! 
